Portland State University

PDXScholar

\title{
Rethinking Autism, Communication, and Community Involvement: Exploring Involvement in Online Communities, Communication Preference, Autistic Identity, and Self-Determination
}

Colleen Anne Kidney

Portland State University

Follow this and additional works at: https://pdxscholar.library.pdx.edu/open_access_etds

Part of the Psychology Commons

Let us know how access to this document benefits you.

\section{Recommended Citation}

Kidney, Colleen Anne, "Rethinking Autism, Communication, and Community Involvement: Exploring Involvement in Online Communities, Communication Preference, Autistic Identity, and Self-Determination" (2015). Dissertations and Theses. Paper 2224.

https://doi.org/10.15760/etd.2221

This Dissertation is brought to you for free and open access. It has been accepted for inclusion in Dissertations and Theses by an authorized administrator of PDXScholar. Please contact us if we can make this document more accessible: pdxscholar@pdx.edu. 
Rethinking Autism, Communication, and Community Involvement:

Exploring Involvement in Online Communities, Communication Preference, Autistic Identity, and Self-Determination

by

Colleen Anne Kidney

A dissertation submitted in partial fulfillment of the requirements for the degree of

Doctor of Philosophy

in

Applied Psychology

\author{
Dissertation Committee: \\ Eric Mankowski, Chair \\ Katherine E. McDonald \\ Greg Townley \\ Christina Nicolaidis \\ Laurie Powers
}

Portland State University

2015 


\begin{abstract}
Autistic individuals experience marginalization and stigmatization, and are often not connected to mainstream services or organizations fostering peer relationships (Boundy, 2008; Jaarsma \& Welin, 2012; Robertson, 2010). Therefore, the accomplishments of the online Autistic community in building a community for selfadvocacy, peer-support, friendships, and identity development (Brownlow \& O’Dell, 2006; Kidney, 2012) are important to recognize, empirically examine, and promote (Blume, 1997a; Davidson, 2008). Utilizing a community-based participatory research approach (CBPR; Israel, Schulz, Parker, \& Becker, 1998) the Academic Autistic Spectrum Partnership in Research and Education (AASPIRE; www.aaspire.org) conducted the AASPIRE Internet Use, Community, and Well-Being Study, and collected data from 151 autistic adults and 173 non-autistic adults. The current study utilized data from the AASPIRE Internet Use, Community, and Well-Being Study on involvement in the online Autistic community, communication preferences, Autistic identity, and selfdetermination. The aims and rationale for the study were to contribute to the small body of quantitative literature on the online Autistic community, including expanding on findings that support positive outcomes of involvement in the online Autistic community (Kidney, 2012), and whether communication preference influences the relationships. The study also sought to examine the fit of an integrated model of associations among involvement in the online Autistic community, Autistic Identity, and self-determination in autistic adults who do and do not prefer online communication. Additionally, the
\end{abstract}


study aimed to promote positive Autistic research and the voices of autistic participants and CBPR partners by challenging common misperceptions about autistic individuals and community/social interactions, understand the impact of communication preference, and inform policy and education designed for autistic individuals.

The study used data to develop a multi-dimensional construct of involvement in the online community as well as explore the Autistic Identity Scale. The main findings included positive relationships between involvement in the online Autistic community and Autistic identity $(r=.33, p<.01)$, as well as between involvement and selfdetermination $(r=.38, p<.01)$ in autistic adults. Exploratory analyses yielded mixed results in the cross-sectional sample that was relatively limited in demographic diversity and small considering the number and type of analyses $(n=324)$. Main findings include no moderating impact of preference to communicate online or population type (autistic or non-autistic) on the significant relationships between (1) involvement in the online community and self-determination or (2) involvement in the online community and Autistic identity. However, exploring the relationships among the variables with structural equation modeling, analyses indicated that no significant relationship exists between involvement in the online Autistic community and self-determination in autistic adults who do not prefer online communication.

Findings indicate the need for further research on the complex impact of communication preference in larger samples of autistic adults. Despite sample limitations and mixed results from exploratory analyses, the findings mostly support the associations of involvement in the online Autistic community with Autistic identity and 
self-determination. The discussion addresses implications of the findings for theory, research, and action. For example, findings may influence how autistic individuals are provided education about and access to new media for fostering community, or lead to the development of online interventions or techniques for the promotion of positive outcomes in autistic individuals. 
The study was funded by Portland State University's Faculty Enhancement Grant to Dr. McDonald. The Burton Blatt Institute and Michael Morris provided additional support for the involvement of community partners.

I would like to express my deep gratitude to the AASPIRE Well-Being team members, including Christina Nicolaidis, MD MPH, Dora Raymaker, MS, Katherine McDonald, PhD, Elesia Ashkenasky, Sebastian Dern, Cody Bosclair, PhD, Amanda Baggs, Steven Kapp, Angie Mejia, MA, Ren Morrill, and Marcie Tedlow. I'd also like to acknowledge and thank the other Gateway Council members for their input through the development of the AASPIRE Internet Use, Community, and Well-Being Study, Morton Gernsbacher, PhD and Jennifer Stevenson, PhD.

Lastly, I would like to thank my dissertation committee members: Eric Mankowski, PhD, Christina Nicolaidis, MD, MPH, Greg Townley, PhD, Laurie Powers, PhD, and especially my advisor, Katherine McDonald, PhD, who has pushed my ideas and persistently encouraged me throughout the dissertation process. 
Table of Contents

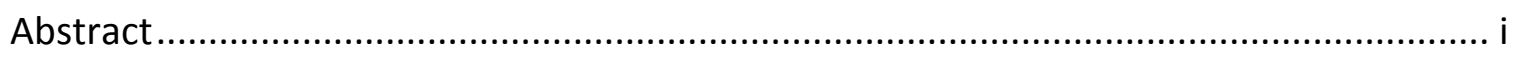

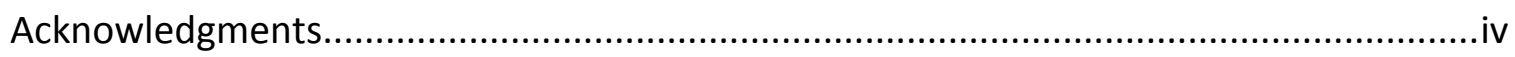

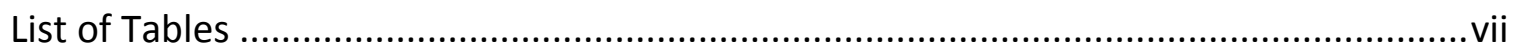

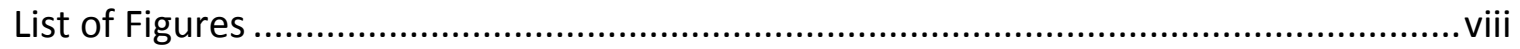

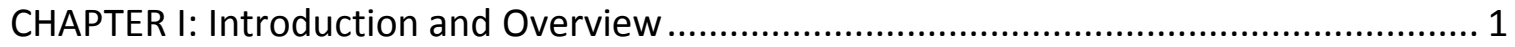

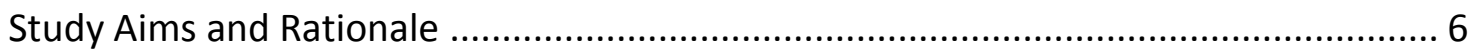

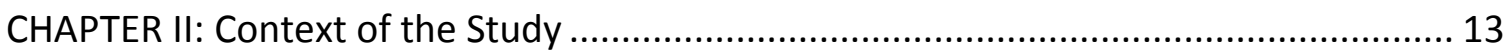

The Academic Autistic Spectrum Partnership in Research and Education .................. 14

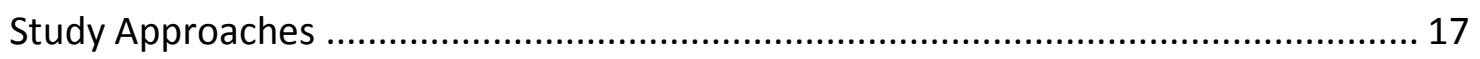

Defining and Characterizing Autism in the Medical Model .................................... 27

The Disability Rights Movement and the Socio-Ecological Model of Disability............ 31

The Autistic Self-Advocacy Movement and the Autistic Community ......................... 38

Re-Defining and Characterizing Autism within the Self-Advocacy Movement ............ 55

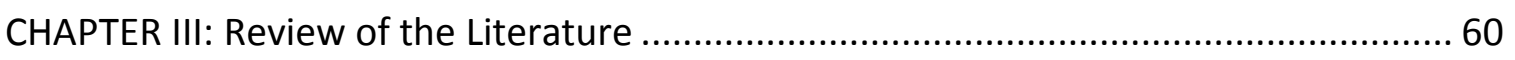

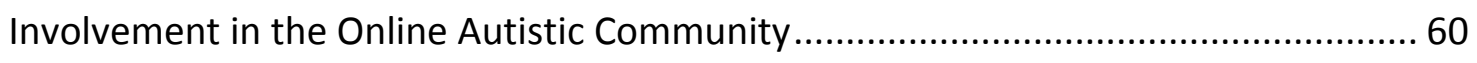

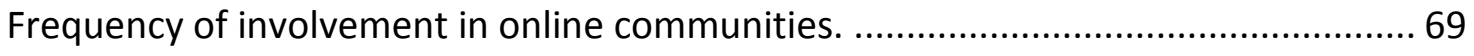

Communication Preferences in the Online Autistic Community .............................. 80

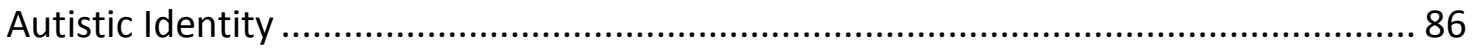

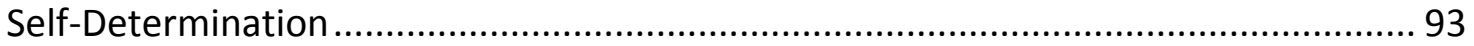

Involvement in the Online Autistic Community, Identity, Community, and Well-Being

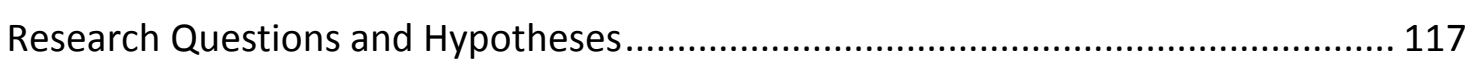

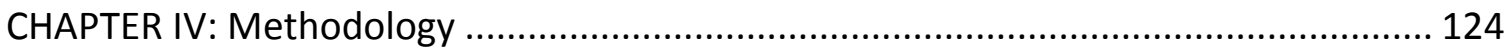

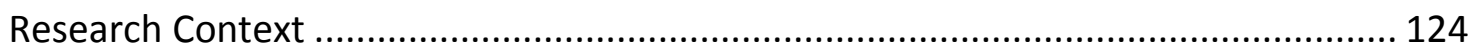

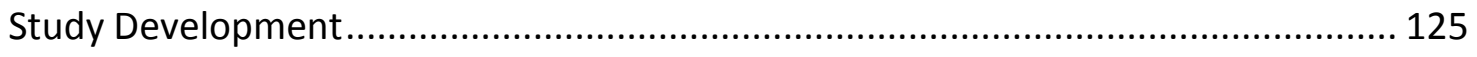

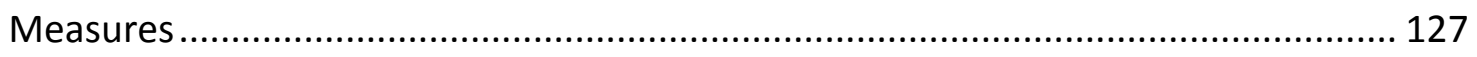

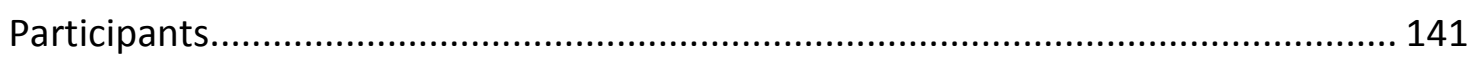

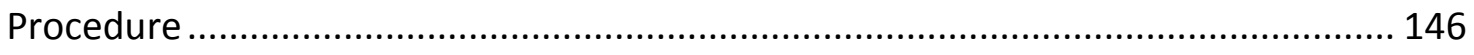

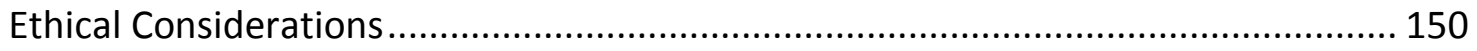

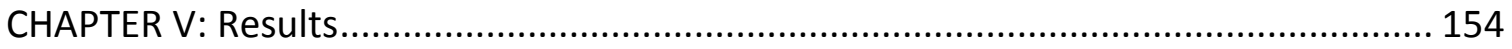

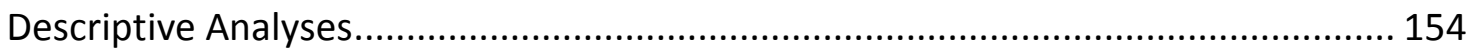




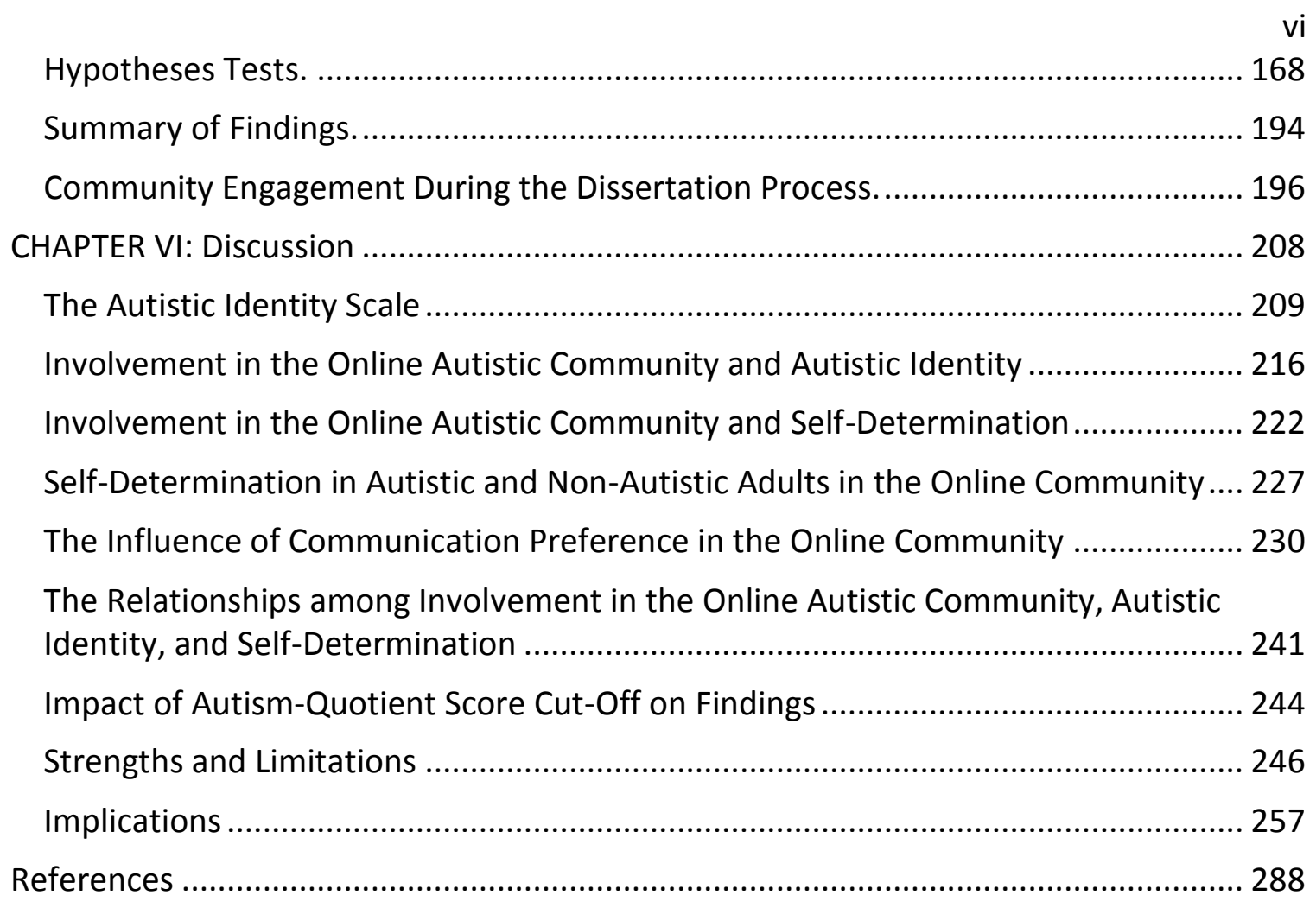


Table 1. Self-determination theory constructs and measures in current study....... 264

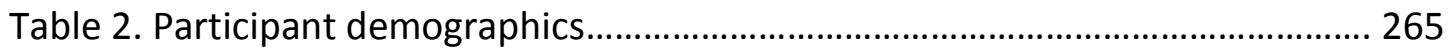

Table 3. Autistic traits and autism spectrum disorder diagnoses.............................. 267

Table 4. Involvement in the online community dimension descriptives and principal

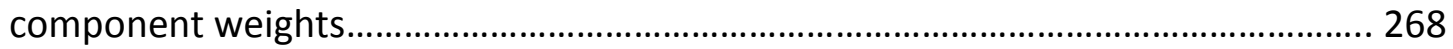

Table 5. Factor loadings of the Autistic Identity Scale Exploratory Factor Analysis.. 269

Table 6. Inter-construct correlation matrix................................................................. 270

0 . Relationship between involvement in the online Autistic community and Autistic identity and their dimensions 271

Table 8. Research questions, hypotheses, and findings. 272

Table 9. Moderation of communication preference on the relationship between involvement in the online Autistic community and Autistic identity 276 Table 10. Relationship between involvement in the online Autistic community and selfdetermination and their dimensions. 277

Table 11. Moderation of communication preference on the relationship between involvement in the online Autistic community and self-determination 278 Table 12. Moderation of communication preference and population type (autistic, nonautistic) on the relationship between involvement in the online Autistic community and self-determination. 279

Table 13. Fit indices for the structural equation models 280 


\section{List of Figures}

Figure 1. AASPIRE's academic and community partner contribution to the CBPR process.

Figure 2. Communication preferences moderating the relationship between involvement in the online Autistic community and Autistic identity.....

Figure 3. Communication preference moderating the relationship between involvement in the online Autistic community and self-determination.

Figure 4. Communication preference and population type (autistic, non-autistic) moderating the relationship between involvement in the online Autistic community and self-determination.

Figure 5. Comparison of relationships among involvement in the online Autistic community, Autistic identity, and self-determination by communication preference

Figure 6. Participants and measures of the Gateway Survey utilized in the AASPIRE Internet Use, Community, and Well-Being Study..... 286

Figure 7. Structural model utilized for the comparison of relationships among involvement in the online Autistic community, Autistic identity, and self-determination in autistic adults who prefer online communication and autistic adults who do not prefer online communication 
CHAPTER I

\section{Introduction and Overview}

The Autistic community formed in reaction to a small number of globally dispersed individuals seeking peer support and friendships (Sinclair, 2005), as well as finding like others to advocate for their rights, much like other disability communities forming self-advocacy groups (Shapiro, 1994). The rise of the popularity of the Internet allowed for the development of the Autistic community and provided a medium for social connections, online journalism, and grassroots organizing (Bagatell, 2007; Silverman, 2008). Also, the Internet became a powerful tool for social action, as well as creating an Autistic culture (Davidson, 2008). Activism such as the "Ransom Notes Affair" (described in Chapter II) anecdotally demonstrates that the online Autistic community provides individuals a place for developing political awareness, connectedness, and a voice strong enough to create social change (Kras, 2009). Through the thousands of first-hand accounts in blogs, forums, list serves, and other websites, the online Autistic community also provides individuals a place for self-expression, gaining knowledge, and developing peer support (Bierer, 2013; Hand, 2011; Rosqvist, Brownlow, and O'Dell, 2013; Sinclair, 2005).

In general populations, the Internet is often used for social engagement and connecting with others (Reich, Subrahmanyam, \& Espinoza, 2012). A number of studies link online socialization to closer relationships, increased social engagement, and wellbeing (Gross, Juvonen, \& Gable, 2002; Kraut, Kiesler, Boneva, Cummings, \& Helgeson, 
2002; Valkenburg \& Peter, 2007). A recent online quantitative study focused specifically on the use of social networking sites (i.e., Facebook, Myspace, or other social network) by autistic adults (Mazurek, 2013). In the autistic adult sample, 79.6\% used social networking sites. This percentage is consistent with research on the general population's use of social networking sites, which indicate that $65 \%$ to $87 \%$ of individuals in the same age range use social network sites (Madden \& Zickuhr, 2011; Sheldon, 2012). The autistic individuals in the sample most frequently used social networking sites for social connection, which is also consistent with general population studies (Reich et al., 2012; Special \& Li-Barber, 2012). Many respondents to Mazurek's survey indicated the communication benefits of online media, and analyses indicated that autistic adults who use social networking sites were more likely to have close friends and report closer friendships. While Mazurek's (2013) study provides new information on the utility of social networking sites for autistic individuals, how autistic individuals use all other online sources of information, communication, and networking and how use impacts the lives of autistics has not been quantitatively examined.

The Internet allows individuals to disclose only as much information as they would like; therefore, specific information on most individual members of the online Autistic community is unknown. However, the community may be defined and characterized by the individuals that participate in and regularly visit the websites, blogs, and forums that serve as the central locations for events, communication, and information sharing. The centralized locations online include the websites, events, blogs, and organizations that branch from a number of websites, including the Autistic Self- 
Advocacy Network (ASAN), The Thinking Person's Guide to Autism (TPGA), Autism Women's Network (AWN), The Autism Acceptance Project (RAAP), and Autism Network International (ANI). The community's members mostly identify as Autistic, regardless of the presence or type of diagnosis. Other members identify as Autistic "cousins," individuals who relate to the Autistic community due to their own social and communication differences (Sinclair, 2005). Some members of the online Autistic community also identify as neurotypical family members, supporters, professionals, or allies of the Autistic community. The term neurotypical is used to describe individuals of the dominant neurological population without reinforcing their privileged position with terms such as "normal" (Walker, 2012).The online Autistic community only consists of the individuals involved in the events, information sharing, social support, or advocacy efforts of the websites, forums, and blogs that connect Autistic individuals. That is, we cannot assume that every autistic individual seeks out and participates in the online Autistic community. Some autistic individuals may not have computer or Internet access or skills, some autistic individuals may seek Autistic communities offline, or some autistic individuals may not seek other autistic individuals for support or community. The online Autistic community can only be defined by those individuals who do participate in the community.

The individuals that compromise the online Autistic community illustrate the diversity within the community. The diversity within the Autistic community reflects the diversity of experiences with autism among individuals, which impacts how individuals think about and identify with autism. The mutual respect for diverse experiences and 
respect for other community members is similar to how the Deaf community describes its' membership (National Association of the Deaf, 2000), which is a community that has been compared to the online Autistic community because of the impact of technology and the development of a community based on self-advocacy and peer support present in both communities (Blume, 1997). Further, the online Autistic community has a history rooted in the disability self-advocacy community (Ward \& Meyer, 1999). The development of the disability community and the online Autistic community is discussed in Chapter II.

The online Autistic community brings together individuals from the broader Autistic community to develop peer support, organize, and lead advocacy efforts for the shifting of attitudes and policies around autism. Autistic individuals seeking online community are interested in forming an identity and community focused on the strengths and challenges of being autistic and to promote positive change in the community and their own lives. Members of the online Autistic community share experiences of exclusion from society, prejudice, a lack of peer support, and misunderstandings by outsiders. The online Autistic community is a part of the larger population of autistic adults, but is distinct from the larger group, who may identify themselves as autistic strictly based upon a diagnosis or characteristics within themselves. The Autistic community's identity is one of a group identity based upon shared history, values, success, challenges, and understanding. Individuals within the community use the label of Autistic as a source of pride and belonging to a community of people, not a descriptor of an aspect of themselves. The current study focuses on the 
online Autistic community. It is important to increase understanding of the involvement in the online Autistic community to understand its impact on the individuals involved.

This unique population experiences marginalization as autistic individuals, with confusion and misinformation existing in society around autism and disabilities that leads to stigmatization (Baker, 2006; Boundy, 2008; Jaarsma \& Welin, 2012; Robertson, 2010; Salomon, 2010; Shore, 2004). Further, many autistic individuals are also often not connected to services or organizations that serve to connect members of other disability communities and minority groups (Ruble, Heflinger, Renfrew, \& Saunders, 2005;

Thomas, Ellis, McLaurin, Daniels, \& Morrissey, 2007). One study comparing the utilization and satisfaction with school and community services for autistic children and children with disabilities through the age of 17 shows triple the odds of reporting difficulty obtaining services for autistic children compared to other children with disabilities (Montes, Halterman, \& Magyar, 2009). With this population often marginalized, stigmatized, disconnected from services, and geographically dispersed, the accomplishments of the community in self-advocacy and fostering a culture and system of support are important to recognize and empirically examine. Little is known about the advocacy efforts of the Autistic self-advocacy movement, or about online advocacy. Understanding the impact of new media on Autistic self-advocacy contributes to the community's call for research that is relevant to the Autistic community and the need for research that will have a positive impact on the community. Further, such research may improve advocacy efforts with increased understanding of how involvement in the community impacts involved individuals. 


\section{Study Aims and Rationale}

This study aims to examine involvement in online communities, Autistic identity, self-determination, and online communication preference. The overarching rationale for the study is to conduct the first quantitative study on positive outcomes of involvement in the online Autistic community. Studying this community is important due to the perceived impact of this relatively new and understudied community that has the opportunity to make positive changes in autistic individuals and the Autistic community. The formation and rapid growth of the online Autistic community was unexpected among individuals that were widely geographically dispersed, lacking knowledge of how to connect meaningfully with one another, often having challenges in social situations and not having opportunities to self-advocate in other environments (Blume, 1997a; Sinclair, 2005). With the emergence of the Autistic self-advocacy movement, autism is viewed by many scholars and advocates as a community and a culture of its own, containing identifiable values, beliefs, and unique knowledge (Silverman, 2008). Having the voices of these individuals heard among the online Autistic community and beyond has created a powerful movement that is reflected in many aspects of Autistic individuals' lives (Blume, 1997b) and well as how autism is perceived by mainstream society (Davidson, 2008). However, little quantitative research is focused on the online Autistic community. Further, studies among general populations show that Internet use and online socializing may increase social capital and friendships (Boase, Horrigan, Wellman, \& Rainie, 2006; Katz, Rice, \& Acord, 2004), which in turn impacts quality of life 
(Kraut, Kiesler, Boneva, Cummings, \& Helgeson, 2002; Putnam \& Miller, 1995). However, no quantitative research examines whether a positive relationship exist between involvement in the online Autistic community and Autistic identity beyond analysis of preliminary data from the current study (Kidney, 2012). Further, no research has examined the relationship between involvement in the online Autistic community and self-determination, nor whether communication preference may impact the relationship.

Therefore, this study has a number of aims. The first aim is to examine the relationships between involvement in the online Autistic community and Autistic identity, and whether communication preference influences the relationship. The rationale for examining involvement in the online Autistic community and Autistic identity includes further supporting and refining findings that suggest positive outcomes of involvement in the online Autistic community by utilizing a larger sample and composite measure of involvement in the online Autistic community. My thesis aimed to examine positive outcomes of involvement in the online Autistic community in order to empirically negate stereotypes of autistic individuals lacking the ability to meaningfully connect with one another online. Involvement in online communities and Internet use for social reasons are associated with well-being and social involvement in the general US population (Boase et al., 2006; Kraut et al., 2002). A shared identity provides a bond between individuals that are marginalized in society (Fine \& Asch, 1988) that may lead to a sense of pride and well-being (Berkman, Glass, Seeman, \& Brisette, 2000; Gill, 1997). Specifically in autistic adults, use of social networking sites is positively 
associated with closer friendships (Mazurek, 2013). Autistic self-advocates share accounts of how the online Autistic community promoted the development of a group identity around autism as well as a personal identity and pride in that identity (Bascom, 2012; Sinclair, 2005; Ward \& Meyer, 1999). A number of qualitative studies indicate positive outcomes of involvement in the online Autistic community and online socializing in autistic adults (Brownlow, 2007a; Burke, Kraut, \& Williams, 2010). Qualitative research also indicates that involvement in the online Autistic community and Autistic identity are dynamically related to one another (Brownlow, 2007a). However, besides examining the relationships within a subset of the current sample (Kidney, 2012), no quantitative study examines the relationships between involvement in the online Autistic community and Autistic identity. A quantitative analysis of a large sample may provide greater reliability of findings supporting relationships between involvement in the online Autistic community and positive outcomes by convergence of similar findings through various methodologies (Morgan, 1998).

The current study is the first quantitative study to examine Autistic identity, possibly also lending convergence of findings with previous qualitative studies and a newly adapted quantitative measurement of the construct. The study utilizes an adaptation of the Disability Identity Scale (Gill, 1997) for autistic individuals; therefore this study also meaningfully contributes to the disability identity literature by examining a new population with the scale. Findings of the study shed light on how the scale compares to the population of autistic adults, and whether the adaptation of a theory of disability applies to autistic individuals. Further, the study provides specific 
psychometric qualities of the scale and subscales, and examines whether it appropriately measures the construct of Autistic identity. No previous studies have examined the properties of the subscales for disability identity or Autistic identity.

The current study next examines the relationship between involvement in the online community and self-determination, and whether communication preference influences the relationship. Rationale for including self-determination in the study includes that Autistic advocates promote a strengths-based approach to research to illuminate positive aspects of the community and promote inclusion (Robertson, 2010). Further, research shows that self-determination has a powerful positive impact on individuals with disabilities, including a higher quality of life and life satisfaction (Cagle, 2006; Lachapelle et al., 2005; Nota, Ferrari, Soresi, \& Wehmeyer, 2007) and employment outcomes (Lachapelle et al., 2005; Shogren et al., 2008; Michael Wehmeyer \& Schwartz, 1998). Also, legislation passed indicating the need to integrate self-determination into policy impacting individuals with disabilities (Baker, Horner, Sappington, \& Ard, 2000; Wood, Karovonen, Test, Browser, \& Algozzine, 2004) and research and development initiatives (Ward \& Meyer, 1999). Yet little selfdetermination research focuses on Autistic individuals, particularly in the online Autistic community. The current study contributes to the literature on self-determination in autistic adults, along with the influence of communication preference on selfdetermination.

A number of the study aims include an examination of the influence of a preference for online communication. Communication preference is important for 
fostering quality relationships and learning, yet autistic individuals may experience social and communication challenges that impact their preference for communication online or by other means (Davidson, 2008). Despite how communication is characterized in autistic individuals, research suggests that computer and Internet technologies may make learning and communication easier in education and therapies for autistic individuals (Rynkiewicz, 2013; Wentz, Nyden, \& Krevers, 2012). Further, qualitative literature and anecdotal information support the impact of computermediated communication on the successes of the online Autistic community in terms of building relationships, community, and advocacy efforts. On the other hand, gaining Internet access, computer and Internet skills, navigating websites, and overcoming any auditory or visual challenges related to websites may be difficult for some individuals (Burks, Pardos, Waddell, \& Nakane, 2000). Therefore, communication preference may impact whether positive outcomes result from involvement in the online Autistic community. Further adding to the complexity of the existing literature, in general populations, preference for online communication is seen as a risk factor for problematic Internet use, loneliness, and depression (Caplan, 2003), but literature has qualitatively supported that the Internet is a safe place to socialize for autistic adults (Davidson, 2008; Ryan \& Räisänen, 2008) and has quantitatively supported no association between social networking sites and loneliness or negative outcomes in autistic adults (Mazurek, 2013). Considering the unique communication preferences which characterize autism, as well as the increasing availability and use of the Internet 
across all populations, there is a need to further explore and understand the impact of communication preference on how online involvement may relate to outcomes.

This study is the first to examine whether preference to communicate online may be influential in the relationships among involvement in the online Autistic community, Autistic identity, and self-determination. This is notable due to the current study examining the construct empirically rather than assume that online communication or communication not online is more effective for autistic adults regardless of preference. That is, supporting the influence of communication preference suggests that communication may be most effective for positive outcomes in autistic adults when communication occurs in the manner most comfortable for the individual. Lastly, this study examines the influence of communication preference on the relationships among involvement in the online Autistic community, Autistic identity, and self-determination in autistic individuals. In addition to understanding the relationships among the pairs of constructions, the rationale for this study aim is to examine whether the relationships have transactional relationships among one another. Examining the complex relationships among involvement, identity, and self-determination may provide evidence of the bi-directionality of the relationships among variables, and how the relationships are impacted by communication preference.

In addition to the literature supporting the need for this study, my thesis study served as a preliminary study examining similar concepts in a smaller subset of the study sample (Kidney, 2012). Despite the smaller sample, the study supported positive relationships between involvement in the online Autistic community and Autistic 
identity, between involvement in the online Autistic community and sense of community, and Autistic identity and sense of community. One of the objectives of this study is to examine the same relationship between involvement in the online Autistic community and Autistic identity in a larger sample, with improvement of the involvement in the online Autistic community variable through use of a multidimensional composite variable. Additionally, non-significant findings between involvement in the online Autistic community and well-being and Autistic identity and well-being in my thesis indicate a need to further examine related positive outcomes in the online Autistic community. More information on my thesis study is discussed in Chapter III.

Following this introductory chapter, Chapter II describes the context of this study, including a description of the research team and research approach for the study, as well as the historical and cultural context of the study, from the characterization of autism in the medical model and how the disability rights movement and Autistic selfadvocacy movement promoted a socio-ecological model of disability that has recharacterized autism today. Chapter III reviews the pertinent literature on involvement in the online Autistic community, communication preferences of autistic individuals, Autistic identity, and self-determination. I then discuss the research questions and hypotheses of the current study. Chapter IV presents the methodology, analyses, and findings of the study. Chapter V presents the findings of the analyses. Lastly, Chapter VI presents the discussion, limitations, and study implications. 
CHAPTER II

Context of the Study

Autistic self-advocates have called for strengths-based research, conducted by or with autistic adults, that addresses ways to improve the quality of life of autistic adults (Robertson, 2010). As an effort to promote respect, accessibility, inclusion, and relevance to the Autistic adult community, a community-engaged approach led to the formation, development, execution, and dissemination of the current study (CDC, 2011). This dissertation utilized data from a study that adhered to the principles of communitybased participatory research (CBPR). In this section I discuss the Academic Autistic Spectrum Partnership in Research and Education (AASPIRE; www.aaspire.org) who conducted the larger study from which data for this dissertation were utilized.

AASPIRE's commitment to CBPR has resulted in a long-term collaborative that has worked together through a number of projects. Next, I specifically describe AASPIRE in terms of the members, organizations, projects, and values that are related to this dissertation. I then describe how AASPIRE adheres to the CBPR approach and how the current study applied a community-engaged approach. Following the discussion on the specific context of this study, I discuss the broader historical and cultural context of the online Autistic community, including the medical model approach to characterizing autism and how the disability rights movement and Autistic self-advocacy movement lead to redefining and re-characterizing autism within a socio-ecological framework. 
The Academic Autistic Spectrum Partnership in Research and Education

AASPIRE is an academic and community partnership that utilizes a community based participatory research (CBPR) approach to research that aims to improve the lives of autistic adults. The academic and community partners collaborate as equal partners to create a team of people with unique skills and knowledge to inform the entire process of research, facilitating co-learning and capacity building among partners as well as research focused on community relevance (Brydon-Miller, 2010; Israel et al., 1998). See Figure 1 for a visual display of the academic and community partners contributing to the research process through the CBPR approach (Nicolaidis et al., 2011). As academic partners ensure the research has scientific value and meets funder priorities throughout the development of the study, the community partners ensure that the research meets community priorities and has community relevance. During the implementation of the study, academic partners ensure safe and scientifically appropriate recruitment and rigor while the community partners ensure accessibility of processes and safe and effective recruitment. As study findings are disseminated, academic partners use findings to build on theory and publish the work while the community partners publicize the findings and help the community with the work.

The AASPIRE team consists of university-based academics, autistic adults, disability service providers, healthcare providers, and family members, and is codirected by an academic and a community member (for more information about AASPIRE partners see aaspire.org). The Steering Committee exists to make quick 
decisions that cannot feasibility be brought to the entire team, as well as making final decisions in the event of disagreement among members of the Community or Academic Councils. The Community Council consists of the community partners of AASPIRE and provides the community voice and input into all aspects of the project. The Academic Council consists of the academic partners of AASPIRE. Each AASPIRE study is lead by two co- principal investigators, one from the Academic Council and one from the Community Council in order to ensure leadership within each project to balance both academic and community perspectives.

Dr. Katherine McDonald and Dora Raymaker, MS, serve as the academic and community co-principal investigators and Dr. Christina Nicolaidis and I serve as coinvestigators of the Internet Use, Community, and Well-Being Study from which data for this study is extracted (the larger study). The community partners that provided support for the Well-Being Study included Elesia Ashkenazy and Cody Boisclair, and Sebastian Dern. Dr. McDonald received a Faculty Enhancement Grant from Portland State University for the larger study. Ongoing support for continued community partner involvement was provided by the Burton Blatt Institute at Syracuse University and Michael Morris.

The AASPIRE partnership exists to bring together members from the academic community and the Autistic community to develop and perform research projects that focus on improving the lives of adults on the autistic spectrum (see www.aaspireproject.org). AASPIRE's goals are to encourage CBPR and the inclusion of autistic people as equal partners, answer research questions relevant to autistic adults, 
and to effect positive change with research findings. The partnership agrees upon these goals, and respects the unique expertise every individual brings to the work. All members of the group are responsible for the formulation of questions, research design, recruitment, materials, analyses, conclusions, and dissemination.

AASPIRE collaborated with the Gernsbacher Lab at the University of WisconsinMadison to conduct the Gateway Project. The Gateway Project serves to enhance research conducted with autistic adults through four values. The four values of the Gateway Project are 1) inclusion: autistic individuals are involved in the development of all studies; 2) respect: all phases of research utilize strengths-based and respectful perspectives and languages; 3 ) accessibility: research participation is made as physically and culturally accessible as possible; 4) relevance: research questions are relevant to the Autistic community. The Gateway Project, by means of the Gateway Survey, identifies eligible participants, recruits them for continuing studies, and collects data on variables to inform subsequent studies. The Gateway Survey collected data on Internet and computer use, demographic information, disability and autistic identity and diagnoses, presence of autistic traits, and future research. Demographic data and data on diagnoses from the Gateway Survey are used to identify eligible participants for AASPIRE's continuing projects, including the present study. I also utilized data from the Gateway Survey in the study as sample descriptors.

AASPIRE's approach to research as a collaborative effort to positively impact the Autistic community is derived from the principles of CBPR as described by Israel (Israel, 2000). Next I discuss CBPR and how the approach is reflected in AASPIRE's processes. 


\section{Study Approaches}

AASPIRE's CBPR approach is central to the formation and functioning of the research team, including the AASPIRE Internet Use, Community, and Well-Being Study, the larger study from which I utilized data for this dissertation. My involvement with AASPIRE and the AASPIRE Internet Use, Community, and Well-Being Study since conceptualization of study variables and design allowed me to engage in the CBPR approach. While CBPR principles would require involvement of the team in every phase of development, my dissertation process involved forming ideas for my dissertation based on community input, then writing and conducting the study independently while checking in with the team and modifying documents and interpretations based on team input. Therefore, while I was involved in the CBPR processes of the AASPIRE Internet Use, Community, and Well-Being Study, this dissertation aligned more accurately with the definition and tenants of community-engaged research. While I planned to utilize CBPR for this dissertation, challenges of the study in engaging AASPIRE team as equal partners informed the retro-fitted description of this dissertation approach as community-engaged. Since CBPR was central to the development of the AASPIRE Internet Use, Community, and Well-Being Study, I discuss CBPR principles and how they apply to AASPIRE here, followed by a discussion of community engagement and how the approach applied to my dissertation.

Community Based Participatory Research. CBPR is an approach in which members of the community are integrally involved in all phases of designing and 
implementing research studies that involve participants from their community (Israel, Schulz, Parker, \& Becker, 1998). CBPR has roots in action research, which was popularized by Kurt Lewin (Lewin, 1946). Action research places social change as the main goal of any research. Since Lewin's initial conceptualization, action research has taken on different names through various incarnations in different fields and research traditions, such as participatory action research (Whyte, 1991) , emancipatory research (Oliver, 1992), and inclusive research (Walmsley, 2004). Despite the high amount of interchangeability these terms possess in theory and practice, AASPIRE specifically builds from the features and principles of CBPR as described by Israel (Israel, 2000; Israel et al., 1998). The AASPIRE Internet Use, Community, and Well-Being Study utilized CBPR principles. First, I describe the tenants of CBPR, then I describe community engagement, the approach I used for this dissertation.

Israel outlines nine key principles of CBPR (Israel, 2000). The first principle indicates that CBPR identifies and works with communities formed around a shared identity. Such communities would have a vested interest in research contributing to social action that would strengthen the community. AASPIRE works with members of the Autistic community, as well as family members of autistic individuals and professionals who agree with the mission of AASPIRE and work with autistic individuals. The research AASPIRE conducts aims to positively contribute to the Autistic community (Nicolaidis et al., 2011). For this dissertation study, I consulted with a subset of the AASPIRE team that identified with the Autistic community and the mission of AASPIRE. 
Israel's second principle requires that CBPR identifies and builds upon strengths, resources, and relationships with the community (Israel et al., 1998). Community members come with a set of skills, connections, and knowledge that are utilized rather than ignored by the professional/academic researchers. In AASPIRE, community researchers have insider knowledge of the Autistic community, including how to reach autistic individuals as peers rather than outside researchers. Most team meetings are conducting via text-based Skype because of the preference of community members to communicate by text rather than in person or over the phone. This meeting format allows for the most effective communication among team members, and for a geographically diverse team to contribute to all communications. It also shows the relevance of this study, as we are an example of the successes of a team who have effectively fostered relationships and accomplished important research primarily via the Internet. The bi-directionality of the research aims and our team functionality are reflections of the CBPR principles that require building upon the community's existing strengths and resources while examining a topic of relevance to the community.

The third principle says that CBPR facilitates collaborative partnerships between all research team members in all phases of research (Israel et al., 1998). Members are considered equal within this partnership, with everyone contributing their individual skills to the project. The members of AASPIRE are included in all major decisions, and informed of any minor decisions made by the project principal investigators or codirectors. Individuals within the group have specific skills to contribute, from copyediting to grant-writing, as well as including or integrating community voice into the 
overarching aims, design, measures, procedures, and recruitment. Due to shared and equal participation in research activities, I use the terms academic or professional researchers to reflect the "traditional" researcher role of someone trained and employed as a researcher, and community researchers to reflect the members of the Autistic community that have taken on positions as researchers, not merely advisors or token team members of AASPIRE.

According to Israel's fourth principle, CBPR aims to benefit all partners and the community by contributing to empirical knowledge in the field that will inform social action (Israel et al., 1998). AASPIRE's projects and collaborations aim to improve health care access to autistic adults, understand the relationship between violence victimization and health in individuals with developmental disabilities, and examine identity, sense of community, social support, and well-being in the online Autistic community. This study aimed to have an impact on how the general public thinks about the Autistic community and justify Internet use and training for positive outcomes of autistic individuals.

The fifth principle emphasizes the facilitation of transferring knowledge and power (Israel et al., 1998). This principle describes how researchers gain insight and a depth of understanding from the collaboration that will lead to stronger, more relevant research that will impact the community, while simultaneously the community researchers gain skills, experiences, and power that will carry over into other parts of their lives. These transactions lead to addressing social inequalities inherent in the marginalized communities represented in such research collaboratives. AASPIRE has 
lead to employment opportunities, development of research skills, and the fostering of empowerment in community researchers. Academic researchers benefitted from an insider perspective to contribute to the validity of instruments, effective recruitment, and accurate interpretation of findings.

Research within the CBPR approach involves a cyclical and iterative process, as specified by Israel's sixth principle (Israel et al., 1998). The research collaborative develops over time and continues to reflect upon problem definition, the relevance of methods and research questions, the efficacy of team processes and roles, and building on study findings. The AASPIRE team began in 2006, and has conducted or collaborated on five studies, all of which involve a cyclical and iterative process. For example, our first project, Healthcare Study 1, focused on understanding health care inequalities in autistic adults. Another project, the AASPIRE Healthcare Toolkit, builds upon findings about health care in autistic adults and aims to have direct social action products through the development and testing of a toolkit to improve health care access and quality for autistic adults.

Israel's seventh principle addresses the examination social issues within the community (Israel et al., 1998). CBPR research utilizes a positive perspective that emphasizes well-being, and an ecological perspective that considers the environment and context of a phenomenon. Much research with marginalized communities, including the Autistic community, has historically focused on deficits rather than emphasizing positive aspects of the community that should be acknowledged and shared for the betterment of the community. AASPIRE focuses on improving the healthcare of autistic 
adults and understanding the positive impact of involvement in the online Autistic community. This positive perspective sheds light on the Autistic community as individuals who do not require pity or help, but have strengths and challenges that can be built upon. The emphasis on an ecological perspective recognizes how phenomena occur within a rich context. Just taking into consideration the historical and social contexts of our work, AASPIRE's research with the Autistic community must recognize the disability rights movement, the rise of the Internet and the online Autistic community, and the societal and professional characterizations of autism.

The eighth principle Israel describes involves CBPR's use of research findings (Israel et al., 1998). Findings from CBPR projects should be disseminated in ways to promote knowledge and benefit all partners. However findings are distributed, they should reflect that all partners contributed equally, and should be respectful to all partners as well as the community. The venue for dissemination should go beyond academic journals and presentations to impact social action, with the language accessibility of the materials seriously considered. AASPIRE partners are invited to contribute to every dissemination effort a member is interested in pursuing. Authorship issues are also addressed with each dissemination effort with the team, with any member that contributes recognized as an author. Since this study tests questions and hypotheses with a new population with relatively new measures, there existed a possibility of finding no positive outcomes or even finding negative outcomes from involvement in the online Autistic community. This may be particularly threatening when negative findings yield from analyses comparing autistic adults to non-autistic 
adults. Such findings may reflect poorly on the Autistic community by empirically suggesting no utility of involvement in the online Autistic community or that autistic individuals have worse outcomes than non-autistic individuals. Negative outcomes, even if supported by the sample data, may damage how people view autistic individuals or the Autistic community, which negates the purpose of conducting CBPR to promote positive outcomes for the community. However, in the event of no positive findings within the analyses, CBPR allows for the unique opportunity to bring findings to community members to re-evaluate the methodology, measures, sampling, timing, and analyses. Rather than solely relying on data-driven interpretation of findings, I attempted to integrate perspectives from community partners in the interpretation of analyses.

The ninth and final principle that Israel describes is that CBPR is a long-term process with a commitment to sustainability. Including multiple individuals and groups may lead to slower research processes than when conducting research independently or in a small group due to the balance of decision-making and transference of power and knowledge in each step. Initially, CBPR projects must work more slowly to understand individual strengths and skills, as well as how to function best as a team. However, initial efforts may result in a healthy and committed partnership over time. While some of the individual members and partnering organizations of AASPIRE has changed since AASPIRE's beginning, many members remain, and the partnerships are becoming wellestablished and growing toward a streamlined, productive CBPR group. 
The CBPR process was modified for the current study within the larger study's approach. This dissertation study utilized some of AASPIRE's guidelines for CBPR, including team approval for my dissertation focus and plans, a number of meetings with team members to inform community perspectives on the Autistic community, approval of how I discuss findings, and approval of all presentations and final products. Every interaction with team members involved communication with AASPIRE investigators and team members with materials modified to be accessible for community partners. However, this dissertation did not fully engage community members as equal partners in the study. Therefore, this study aligns more closely with a community-engaged research approach. Community engagement is defined next, as well as how the current study utilized the approach.

Community-engaged research. While the overarching study from which I utilized data for the current study adheres to the principles of CBPR, this dissertation utilizes a community-engaged research approach. Community engagement is an approach to research and practice in health promotion defined as "the process of working collaboratively with and through groups of people affiliated by geographic proximity, special interest, or similar situations to address issues affecting the well-being of those people" (CDC, 2011, p. 3). Researchers applying the approach of community engagement collaborate with organized groups, agencies, institutions, or individuals with a special interest in utilizing research to promote well-being in the community. 
Research may apply community engagement on a continuum on community involvement, impact, trust, and communication that ranges from basic outreach to the community at the lowest level to shared leadership of the research with the community at the highest level (CDC, 2011). Similarly, participatory action research literature describes levels of engagement with the disability community ranging from engaging an advisory board at the lowest level to having true partnerships in the research team with community members (Balcazar, Keys, Kaplan, \& Suarez-Balcazar, 1998). The CBPR processes utilized in AASPIRE, including the larger study from which data for this study were utilized, may be considered an approach to research the highest level of community engagement or participatory action research, where the final decisions are made by community members, strong partnership structures are formed, and strong bidirectional trust and relationships are built.

This dissertation involved a number of steps conducted with the AASPIRE team. First, the team worked with me to identify relevant research aims and questions through a brain-storming meeting in which we reviewed the available data from the broader Well-Being Study and discussed the possible directions I could take with a dissertation study, as well as the implications of the potential study aims and questions. I took the ideas from the meeting and formulated dissertation aims and research questions with my knowledge of the related academic literature and my familiarity with the statistical analyses possible in the current sample. I next proposed my dissertation ideas to the team who provided approval of the dissertation aims and research 
questions. After writing a dissertation proposal, I received feedback and approval on the overarching purpose and aims, proposed hypotheses and analyses, and potential implications. After my proposal was approved by AASPIRE and my dissertation committee, I conducted the proposed analyses. I then met with AASPIRE team members who provided input in interpreting findings and connecting findings to the community. After writing an early dissertation draft, I met with the team to present a summary of my results, discussion, and implications for feedback. Lastly, I met with the team to debrief the experience of involvement in my dissertation, specifically reflections on the use of CBPR and community engagement in the study.

During all phases of my dissertation study, communication mostly occurred online to respect the preference of the community partners and involved a small stipend depending on the availability of funding and time required for feedback. I had little communication with the AASPIRE team between these steps of the process. While the process involved community input formulating dissertation ideas and feedback at each major step of the dissertation process, the dissertation does not reflect a bidirectional relationship or shared leadership of the study. Rather, the level of community engagement may be more accurately described at the level of consultation, where feedback was elicited and taken into account, the community shared information with me to inform the study, and we developed connections but not partnerships (CDC, 2011). I discuss the self-reflections and community partner feedback on the successes 
and shortcomings of the community engagement in the Results section entitled, "Community Engagement During the Dissertation Process."

After describing the context of this study through the CBPR principles and community engagement, the context of the community is critical for understanding the study. I next explain the medical or traditional definition of autism, followed by the history of the disability rights movement and the Autistic community contributing to a different way of defining and characterizing autism.

\section{Defining and Characterizing Autism in the Medical Model}

Autism has garnered much media attention in the past decade, due in part to researchers, practitioners, and non-professionals falsely claiming that there is an autism epidemic, and that autism is caused by environmental toxins or vaccines (Grinker, 2007). The politicized debates on defining autism, the causes of autism, and the goals of educators, researchers, and practitioners in the field of autism are highly polarized and publicized (Solomon, 2008). Different characterizations of autism come from various traditions of medicine, but also social sciences, the media, and autism organizations (Murray, 2010). In order to express definitions of autism and autism research beyond the "typical" autism conceptualization (Yergeau, 2010), or the traditional medical conceptualization, I first provide a definition and brief background about autism as a diagnosis. This definition and brief background describes an acontextual and deficitsbased view of autistic individuals that is not how many self-advocates, or the AASPIRE team, define or characterize autism. I then discuss the history of the Autistic self- 
advocacy movement that contributed to autism as a difference in how persons live, think and communicate. Then I discuss a description of the definition and characterization of autism that AASPIRE uses.

Autism is characterized by impairments or differences in communication and social interaction (National Institute of Neurological Disorders and Stroke, 2009). Eugen Bleuler first used the term 'autism' to describe a branch of schizophrenia in 1911 (Brownlow, 2010). Kanner (1943) then reintroduced the term into psychiatry as a unique syndrome. A year later, Hans Asperger (1944) independently presented clinical case studies detailing the deficits and special abilities of a small group of boys. Despite the decades that have passed since these initial studies of autism, a single definition and diagnosis of autism is still not universally accepted. The Diagnostic and Statistical Manual of Mental Disorders published its first two editions, the DSM-I (1952) and DSMII (1968), which identify autism as a symptom under the diagnosis of schizophrenic reaction, childhood type label; the DSM-III (1980) introduced autism (called infantile autism until 1987) as a separate diagnostic category with six symptoms that all must be present for a diagnosis. Autism was not considered a spectrum disorder containing subtypes until the DSM-IV was published in 1994, which includes a diagnosis based upon existence of some of a variety of possible symptoms. The subtypes include autistic disorder, Asperger's Disorder, Rett's Disorder, Childhood Disintegrative Disorder, and Pervasive Developmental Disorder Not Otherwise Specified (PDD-NOS). These diagnoses are all broadly termed as autism spectrum disorders (ASD), considered to exist on a spectrum of disorders with defining features of difficulties in socialization, difficulties in 
verbal and nonverbal communication, and restricted and repetitive behaviors (American Psychiatric Association, 2000). The DSM-V (American Psychiatric Association, 2013) revised the diagnostic criteria of autism spectrum disorders by collapsing the subtypes into one unifying diagnosis of autism spectrum disorder characterized as a spectrum of social communication differences and repetitive/restricted behaviors.

Contemporary research and diagnosis characterizes autism as a 'triad of impairments' that interact with one another uniquely in every individual (Wing, 1997). The triad includes social development, language development, and rigid and repetitive behaviors. The disorders are considered on a spectrum and therefore manifested differently in every individual. Therefore, other characteristics of the disorder are not universal to those on the spectrum. However, common characteristics are associated with ASD (National Institute of Child Health and Human Development, 2005). These characteristics include a range in verbal communication abilities, with some individuals utilizing minimal oral language. Other forms of communication or body language may be challenging, such as pointing, eye contact, or smiling. Expressing emotions or understanding others' expressions of emotions may be challenging, as well as the length or timing of any social interactions. Autistic individuals may exhibit repetitive behaviors or stringent routines and attachments. Physical movements such as rocking, hand or finger flapping or twisting, or whole body movements may occur, and autistic individuals often describe feelings of sensory overload, or sensitivity to multiple physical or mental stimuli, such as bright lights or large crowds (Filipek et al., 1999). 
The Center for Disease Control and Prevention (2004) considers autism a developmental disability, a life-long condition starting before the age of 22 characterized by mental and/or physical impairments that impact self-care, comprehension, language, skills, learning, mobility, capacity for independent living, economic self-sufficiency, and/or the ability to function without assistance (DHHS: US Department of Health \& Human Services, 2009). While little research exists that examines disparities specifically in autistic adults, physical and psychological health disparities exist in persons with developmental disabilities (Havercamp, Scandlin, \& Roth, 2004; Sutherland, \& lacono, 2002) that may also be true in the autistic adult population (Nicolaidis et al., 2011; Nicolaidis et al., 2012).

A longitudinal study of 120 diagnosed autistic individuals found that as adults, $90 \%$ of the participants had social challenges, $35 \%$ achieved near-normal fluency of oral language and $29 \%$ achieved near-normal comprehension of oral language (Ballaban-Gil, Rapin, Tuckman, \& Shinnar, 1996). Another study suggests that autistic adults face many challenges in having close friends, living independently, or obtaining permanent employment (Howlin, Goode, Hutton, \& Rutter, 2004). However, little research exists on the impact of social skills in different contexts, with the majority of autism research focusing on the diagnosis and childhood of individuals (e.g., Bradley, Summers, Wood, \& Bryson, 2004; US Department of Health and Human Services Interagency Autism Coordinating Committee, 2009) or with outcomes of autistic adults as reported by parents and siblings (Farley et al., 2009). Bishop (1989) describes the importance of a lifespan approach for children with ASD. Bishop argues that focusing on childhood 
diagnoses and research may result in the misrepresentation of autism as a disability resulting in static, unchanging behaviors and abilities as well as the perception of autistic individuals as eternal children. Furthermore, the research and literature in the field of autism is often influenced by the perspectives of researchers, parents, teachers, and professionals - rather than autistics - and tend to define autism and research within a deficit-oriented model (Biklen, 2005; Singh, Illes, Lazzeroni, \& Hallmayer, 2009). Through this framework, research that informs opinions, therapies, and treatments focuses on something being intrinsically wrong with being autistic or wrong with how autistic individuals experience the world (Boundy, 2008). This deficits-oriented paradigm can further contribute to marginalization and physical and psychological disparities among autistic adults.

The next section provides historical context for the disability rights movement, which began to counter the deficits-oriented paradigm within broader disability communities by reframing how individuals with disabilities were viewed. The disability rights movement was a precursor to the Autistic self-advocacy movement through which new conceptualizations of autism were born.

\section{The Disability Rights Movement and the Socio-Ecological Model of Disability}

The disability rights movement yielded notable progress in the past decades to address marginalization, yet persons with disabilities continue to experience unique obstacles in achieving inclusion in society. Here I provide a short historical context for the disability rights movement and the disability self-advocacy movement, since the 
Autistic self-advocacy movement can be considered a subset or outgrowth of the movement made possible by the advancements of the disability community.

Before the 1970's, a medical model of disability was applied that is still accepted by many today, claiming any communicatory, social, and behavioral differences were considered medical in nature, with a focus on the individual and attempts to rehabilitate or normalize the person according to mainstream standards while encouraging segregation from society (Brisenden, 1986). In the late 1940's and early 1950's, parents of children with disabilities began to take action against the lack of appropriate care given to their children by many professionals, social services, and educators (Shapiro, 1994). Parents formed organizations such as the United Cerebral Palsy Association, the Muscular Dystrophy Association, and ARC to advocate for such services for their children. By advocating for services that cater to the unique needs of their children in education, medical settings, therapies, social services, and community inclusion, the public was exposed to individuals with disabilities as a group of people deserving of rights, thus beginning to move forward a disability rights movement in the US.

Originating in the first organizations run by parents to advocate for the rights of their children with disabilities, individuals with disabilities began recognizing that they should have power as a large community. Since individuals were segregated by society and stigmatized under the unjust frame of the medical model, individuals with disabilities themselves often adopted the same opinions towards individuals with disabilities. However, with growing value being placed on individuals with disabilities through advocating for their rights and new opportunities to pursue opportunities, such 
individuals were developing more positive feelings towards themselves and the disability community, impacting the need to advocate for their own needs. Individuals with disabilities directed their growing militancy to eliminating stereotypes, inequalities, and exclusion from society (Shapiro, 1994). The community likened the minority status individuals with disabilities experience to other societal minorities such as racial minorities or struggles faced by women (Gordon \& Rosenblum, 2001). Problems of employment, healthcare, discrimination, abuse, and fair housing that are often discussed regarding minority groups overlap the issues faced by people with disabilities. As a means to addressing inequalities, the early disability rights movement began with advocating for independence in living and decision-making, self-sufficiency, mainstreaming or integration into broader society, and eliminating the medical model of disability by adapting a socio-ecological model of disability framework (Shapiro, 1994).

By shifting the lens through which society views people, empowering the disability community started to bridge the disparities of this social minority group and create equality (Pledger, 2003). The terms impairment and disability refer to different concepts, with impairment referring to the condition of losing or having a difference in physiological or psychological function (Oliver, 1996). Disability refers to the resulting loss or limitations of opportunities due to the interaction of impairment and the environment. The socio-ecological model of disability asserts that as the environment becomes more supportive and inclusive, individuals and the disability community gain more control and influence over the environment (Fawcett et al., 1994). The World Health Organization (2002) describes the International Classification of Functioning, 
Disability, and Health (ICF), which is a similar model wherein a person has a specific level of capacity that may or may not be realized by their level of performance in a specific environment, with functioning describing bodily functions, activities, and structures that interact within systems. The paradigm that is described by such models and definitions is referred to as the socio-ecological model of disability. The socio-ecological model of disability rejects the medical model and views disability as a function of society's shortcomings and systemic barriers (Tate \& Pledger, 2003). Despite widespread acceptance and utilization of the socio-ecological model of disability (Barnes, 1998), some critics of the model feel it does not depart enough from the medical model to address the varied perspectives and experiences of individual members of the disability community. For instance, the socio-ecological model still assumes normality, achievement, participation, employment, independence, conventionality, and conformity as successes or ideals to strive towards in advocating for rights rather than acceptance and appreciation for the differences in ideals among individuals (Shakespeare \& Watson, 2002; Shapiro, 1994). Despite differences in how to characterize the epistemological stance of the movement, the disabilities rights movement grew from the rejection of a medical model that oppressed and stigmatized individuals with disabilities.

The growth of the ideas put forward with the socio-ecological model of disability is in tandem with the disability rights movement's actions and growth. A number of historical moments in the disability rights movement occurred in the early 1970s, such as in 1972 when the Center for Independent Living (CIL) formed from the Physically 
Disabled Students' Program at Berkeley by Ed Roberts and John Hessler. Roberts and Hessler demanded that the CIL should be run by persons with disabilities who were advocates for integration into society and fighting the oppressive medical model of disability. Judy Heumann started a self-advocacy organization in the early 1970's as well, called Disabled in Action, after she was denied a teaching certificate on the basis that she would not be able to help students in emergencies or perform other required duties of a teacher due to her disability (Shapiro, 1994).

The growing power of the political disability rights organizations forming across the country organized a sit-in in 1977 to retain Section 504 of the Rehabilitation Act of 1973 which authorized federal aid to be spent on individuals with disabilities and made discrimination based on disability illegal (Shapiro, 1994). The Department of Health, Education, and Welfare estimated that compliance would cost billions of dollars, so they stalled the issuance of the final regulations. The American Coalition of Citizens with Disabilities began demonstrating in Washington, DC, then spread to San Francisco, with individuals, some in life-threateningly dangerous situations away from medical and support needs, occupying the UN plaza in San Francisco for 25 days. The San Francisco sit-in marked the political coming of age of the disability rights movement, and supported the community's idealism, political power, and growing beliefs in changing how individuals with disabilities are viewed in society. Further, the sit-ins helped CIL gain national recognition, with 300 starting across the country within the next 10 years. Such expansion of ideals spread the new philosophy and socio-ecological model of the disability rights movement to a wider disability audience, as well as to family members 
and professionals. A new philosophy of no one knowing more about individuals with disabilities than themselves spread, as well as a mantra of "nothing about us without us."

In addition to the actions and organization of groups primarily compromised of individuals with physical disabilities, the Deaf community began gaining momentum in the 1980's (Shapiro, 1994). Like other disability communities, the Deaf community was experiencing a growing sense of oppression in society. Much like the Autistic community, social and technological trends has lead to an increase in membership in Deaf organizations, groups, and self-advocacy efforts. The first television show to include closed captioning was broadcasted in 1971, with the first news broadcast with closed captioning occurring in 1973 . Telephones became more accessible, leading to a greater ability for deaf individuals to connect when not communicating face-to-face, as well as organize for self-advocacy. Further, ASL was becoming an acceptable language, with interpreting growing as a profession. Such events not only impacted the accessibility of entertainment and had an impact on inclusion of deaf individuals in society, it also lead to a more informed and connected Deaf community. Shapiro (1994) also points out that the individuals active in the 1980 's Deaf self-advocacy movement were members of the generation born in between the civil rights movement and the Vietnam era, placing them in households and schools that fostered attitudes and skills that were militant and action-oriented when seeking social change. Individuals displayed such militancy during the protest at Gallaudet University, a university for deaf students, in 1988. A hearing president resigned at the university, and news of the next president 
also being a hearing individual spread throughout the student population. This appointment was counter to the goals of the disability rights movement, creating a revolt via student rallies and walk-outs that resulted in the hearing presidential candidate to resign, a deaf president appointment, and deaf individuals appointed to make up the majority of the board of trustees. Not only did this incident mark what was seen as the Deaf community coming of age, but also society first understanding the implications of disability community actions as civil rights advocacy, with widespread tactics and language used that mirrored civil rights movements.

The Deaf community was just one of the disability communities that experienced a separate rights movement and self-advocacy awakening within or alongside the disability rights movement. Individuals with intellectual disabilities were another segment of the disability community that was marginalized or less represented in the disability rights movement. Institutionalization for individuals with intellectual disabilities was widespread and standard until the late 1940's deinstitutionalization of individuals with psychiatric disabilities began. During the rise of the disability rights movement and the deinstitutionalization of individuals with psychiatric disabilities, such institutions were uncovered as horrific living conditions likened to prisons for all individuals. The disability rights movement was exposing society to what their family members experienced via the accounts of advocates and researchers, such as the photographic essay "Christmas in Purgatory" by Burton Blatt and photographer Fred Kaplan (1974). American society adapted a sociological approach to deinstitutionalization from Scandinavia where individuals were placed in non- 
institutional settings and given the opportunity to experience "normalization," the dignity of risk, and choices in matters that impacted their own lives (Dybwad \& Bersani, 1996). Bengt Nirje, the director of the Swedish Parents Association for Mentally Retarded Children, launched such an approach to self-advocacy in 1968 by asking the children for input in developing their daily program, with the novel assumption that they understood what they would like to do more than the professionals that work with them. Once individuals introduced this approach to the US, self-advocacy groups for individuals with intellectual disabilities started organizing and spreading across the country, including chapters of People First (in 1974) and SABE (in 1990).

The disability self-advocacy movement provides a context through which the Autistic self-advocacy movement began. The self-advocacy movement of the Autistic community, like the Deaf self-advocacy movement and self-advocacy movement of individuals with intellectual disabilities, is considered within the broad context of the socioecological model of disability and the disability movement (Chamak, 2008). Like activists in the disability rights movement, Autistic self-advocates work toward changing the structural inequalities that prevent individuals from meaningfully participating in society and reaching personal goals (Boundy, 2008; Broderick \& Ne'eman, 2008; Hurlbutt \& Chalmers, 2002). The Autistic self-advocacy movement and the reconceptualization of defining and characterizing autism are described in the next section.

\section{The Autistic Self-Advocacy Movement and the Autistic Community}


The disability rights movement gained a large amount of support and legitimacy by the early 1990s, as evident through the Americans with Disabilities Act of 1990. While the disability rights movement theoretically encompassed protecting the rights and promoting the well-being of autistic individuals, autism was a relatively unknown and a rare diagnosis that was not explicitly acknowledged by the movement at the time (Shapiro, 1994). When the DSM-III (1980) recognized autism as a separate diagnostic category, individuals began to receive the diagnosis and could identify as autistic. However, the DSM-III had stringent diagnostic criteria and a narrow characterization of autism that contributed to stigmatizing beliefs about the abilities and characteristics of autistic individuals. While individuals were diagnosed with autism, the number of individuals receiving the diagnosis was small and geographically dispersed, with little understanding of their lives, no community of like individuals to connect with, and stigma around their behaviors and new label that made creation of support systems difficult (Ward \& Meyer, 1999). Autistic individuals that did not receive a diagnosis with the DSM-III remained hidden and unable to find like others due to the inability to put a name or find a community on something inexplicable.

Though any diagnosis comes with its own stigma and issues, individuals with a label of autism do receive more attention in educational systems or other social systems (Chamak, 2008). In fact, educational diagnoses or assessments of autism became common in the 1990s, in which a non-medical professional within the educational system determines certain educational accommodations or supports should be provided for a child. These diagnoses brought support needs to the attention of children, parents, 
and professionals, leading to more awareness of autism and larger numbers of autism diagnoses. The emergence of a self-advocacy group was in part due to the new diagnostic criteria and wider diagnoses across non-medical professionals, because a better understanding of autism through definitions (or a reaction to differences from the definitions) allowed individuals to begin on the path of self-advocacy (Brownlow, 2007a; Ward \& Meyer, 1999). For these reasons, it was a struggle to develop relationships or a community with one another before published diagnostic criteria for autistic individuals.

Donna Williams (1992, 1994), an Autistic self-advocate, describes the struggles to find anyone to relate to or that experiences the world in the way she did prior to an autism self-advocacy movement. The few individuals she comes across that she sees as "alien" like herself generally had never heard of autism, think autism is a serious mental disorder that results in institutionalization, and certainly do not identify as autistic. They also share with Williams a sense of alienation and loneliness due to feeling as though they are not in "the world." Finding these individuals coincidentally at first and eventually through the successes of her autobiographies allows Williams to find belonging and acceptance where it was absent in her relationships with non-autistic individuals. Another Autistic self-advocate wrote: "I believe all persons with autism need the opportunity to become friends with other autistic people. Without this contact we feel alien to this world. We feel lonely. Feeling like an alien is a slow death. It's sadness, self-hate, it's continuously striving to be someone we're not. It's waking up each day and functioning in falsehood." (French, 1993 as quoted in Sinclair, 2005). 
Before the widespread use of the Internet, autistic individuals had few places to seek out peers. Autism conferences in the 1980 s and early 1990 s were organized by and for non-autistic professionals and non-autistic parents of autistic children. Such conferences were hostile environments, both sensory and emotionally, for autistic individuals due to the number of people, noises, lighting, and the overall negative depiction of autism. While the Internet had potential as an autistic-friendly environment, the Internet was not widely available or accessible until the mid-1990s. Jim Sinclair (2005) describes what could be the earliest incarnation of the Autistic community as a small group of autistic individuals who first contacted one another via a pen pal list maintained by a non-autistic parent-run organization.

The disability self-advocacy was growing, and a small number of autistic individuals began to attend more conferences to understand and be involved in decisions that impact their lives. During the Autism Society of America national conference in 1991, the organization approached Jim Sinclair and other autistic individuals to be in an advisory committee. The organization promised them a voice in the Autism Society of America through their new role, support for creating a selfadvocacy organization, and a column for self-advocacy issues in their newsletter. The organization did not honor these promises, thus beginning a history of tension between the self-advocacy movement and autism organizations run by parents and professionals. The unfulfilled promises also stimulated a need for self-advocates to branch out from their dependence on outsider organizations and begin their own network. 
In 1992, Donna Williams was in the US to promote her first autobiography Nobody Nowhere (1992), and visited Kathy Lissner Grant and Jim Sinclair, other Autistic self-advocates with whom she had been in contact with via mail and fax. Williams later described the connection she felt during this visit: "Despite thousands of miles, our 'our world' concepts, strategies, and experiences even came down to having created the same made-up words to describe them. Together we felt like a lost tribe. 'Normal' is to be in the company of one like one's self" (Williams, 1994). Despite traditional views of autism, Williams also described the ease of communication when with other autistic individuals: "[l am]...able to communicate with someone in my own language... meaning flowed freely and easily." Williams and Sinclair both describe feelings of Autistic peer support in which the three individuals supported one another managing tasks of everyday life during the visit. The small group felt strongly connected and supported by other Autistic adults and they felt other Autistic adults may benefit from peer relationships as well.

Williams, Grant, and Sinclair decided to start their own organization led by Autistic adults rather than continue to support and depend upon organizations run by non-autistic professionals and parents. The purpose of Autism Network International (ANI) was originally to help Autistic adults find one another and create a network for news and events. ANI also sought to be a mutual aid self-help group for individuals to share their experiences to counter offensive and offensive portrayals of autism that exist, and to promote well-being in their members. They named their organization Autism Network International, in part to reflect that they did not think the network 
would grow into a community. Their connections with one another and with other Autistic individuals occurred on a person-by-person basis, one at a time, and the idea of an organized community based on relationships, advocacy, and support did not seem like a meaningful concept for autistic individuals.

Due to the geographically dispersed members of ANI, the network established distance communication via a printed newsletter and a pen pal list for private personto-person communication. The newsletter, edited by Jim Sinclair, was called Our Voice and began distribution in November of 1992. The first distribution of Our Voice had 15 subscribers who were mostly non-autistic parents of autistic children. ANI received hostile feedback from non-autistic parent and professional organizations after the distribution of the first issues of Our Voice. The Autistic base of the organization sought to reach out to new Autistic individuals. Sinclair, who was the only autistic person on a parent and professional online autism mailing list, used this forum to reach out and find other Autistic individuals to join ANI.

As membership in ANI slowly grew, the network began to take shape, with members seeing commonalities among one another. One commonality members noted was how many individuals in the group carried the label of "high-functioning autism," often given to autistic individuals with a high degree of verbal language regardless of challenges in other areas of life. Jim Sinclair describes the inaccuracy of this label: "Notwithstanding our various difficulties with sensory processing, social comprehension, emotional modulation, employment, adequate self-care, household management, and assorted other life skills, we were all considered to be 'high-functioning.'... But we had 
all fit descriptions of 'low-functioning' autistic people when we were younger. We all recognized commonalities between ourselves and autistic people who were still considered 'low-functioning.' We also recognized abilities and strengths in many autistic people who just didn't happen to share our skills in using language." Sinclair, Williams, and many other autistic individuals from the movement are publically criticized as not being autistic or being too high-functioning to represent the voices of autistic individuals who do not verbally communicate. While ANI's membership may appear to outsiders as 'higher-functioning' due to the supportive environment allowing functionality at a given moment, members identify with others on the autism spectrum who could not verbally communicate and have greater support needs. Therefore, they advocate for all autistic individuals regardless of their ability to participate in ANI. Further, they allow parents and professionals to be a part of their network in order for education and change in systems to occur so that they may affect individuals who are unable to participate on their own. Parents and professionals, however, are not allowed on the person-to-person private pen pal list that was specifically designed for Autistic individuals for peer support.

These beginnings of a community, along with the push-back from non-autistic parents and professionals, was mirroring other disability communities and the broader disability rights movement (Dybwad \& Bersani, 1996). Autistic self-advocates were recruited or finding ANI themselves one by one, slowly growing and strengthening the organization. The community grew through personal connections. Therefore, the relationships and support formed took higher importance than any community 
organizing or social action goals. ANI unintentionally grew into a community rather than an organization aimed at self-advocacy or political action. ANI gained presence at autism conferences by setting up a booth for their network that became a meeting place and sanctuary for the autistic attendees in hostile conference environments. Individuals would travel and stay together at conferences in groups since few had the money for conferences. They fostered close relationships through such events. In tandem with the rise of ANI at conferences, other Autistic self-advocacy and peer support groups formed throughout the early 1990s (e.g., Autistic Adults Coming Together; Bagatell, 2010). Community members started to connect more through the growing use of the Internet.

ANI and other sprouting self-advocacy groups directed autistic adults to the nonautistic parent and professional mailing list that ANI members and other Autistic selfadvocates infiltrated. At first, Autistic individuals posted their experiences and wrote narrative accounts of their lives, and then parents of autistic children would respond with questions or begin conversations to try to gain a better understanding of their autistic children. As more autistic individuals became part of the mailing list, it became more focused on peer communication, branching into private email correspondence, which led to Autistic individuals forming and maintaining personal relationships online. Autistic cultural influences became part of the community and Autistic individuals' lives online and offline, marking greater widespread appreciation for the growing community and their unique traditions and interests.

At the International Conference on Autism in 1993, Sinclair presented his paper "Don't Mourn For Us," where he challenged the mostly non-autistic audience to reframe 
their thinking about autism as a tragedy (Sinclair, 1993). This presentation drew in much attention and new members to the community, including non-autistic individuals with other significant social and communication challenges that related to the Autistic community. These individuals became known as "cousins" to the Autistic self-advocacy community. A broader self-advocacy movement that addresses the rights and wellbeing of all individuals with any neurological differences became known as the neurodiversity movement. The Autistic self-advocacy movement and the neurodiversity movement share many of the same values and goals. The neurodiversity movement is commonly used interchangeably with Autistic self-advocacy movement, despite the broader population included with the term "neurodiversity."

In addition to Sinclair's presentation at the International Conference on Autism drawing in new members and attention, the presentation also stirred up conversation in the growing online forum. After the early beginnings of the small Autistic community being online, there was a sharp increase in online Autistic presence following the 1993 International Conference on Autism due to an increased awareness of the mailing list as well as the excitement of the individuals who had recently attended the conference and made connections with one another. The forum became over-run by reflections on the conferences, inside jokes, and conversations furthering the personal relationships begun or invigorated by the conference. Non-autistic parents and professionals were annoyed by such posts that did not have relevance in their lives or suit the purpose of nonautistic individuals utilizing the forum. Disagreements broke out about what topics were appropriate to discuss. This disagreement was named the "Snore Wars" because the 
conflict peaked when conference attendees used the forum to humorously reflect on someone who fell asleep and snored during a conference presentation.

In reaction to the tension between the Autistic self-advocates and the nonautistic parents and professionals, ANI decided to create their own space online. ANI launched the ANI-L list serve in 1994. The list serve created its own rules and divisions to establish an "Autistic space" online that allowed Autistic individuals to communicate in the ways best for the community. Non-autistic parents and professionals who were members of ANI were allowed to join the specific parent list and participate in any forum of the online community as long as they respected the communication and space as Autistic.

In 1998, Autistic individuals founded autistics.org as one of the first Autistic websites dedicated to being a database of information and resources by and for autistic individuals. It used the motto "The Real Voice of Autism" in reaction to non-autistic organizations run by parents and professionals often claiming to be the voice of autism. Autistics.org became a major web hub for Autistic advocacy, giving self-advocates an accessible source from which to gain information and organize. Autistics.org also later started a group on Second Life called the Autistic Liberation Front, focused on Autistic self-advocacy issues.

The emergence and popularity of blogs in the late 1990s gave Autistic selfadvocates a place to share their first-person accounts of their experiences and lives, as well as read others' and connect over shared experiences and interests. Thousands of Autistic self-advocates began communicating through blogs, which grew increasingly 
user-friendly and popular. Further, other online resources for social and informational sources grew in popularity, such as social networking sites, online forums, real-time chat groups, list serves, and other community-building sites. Simultaneously, online journalism was on the rise, with individuals with computers and Internet able to readily and freely access news. With the ability to access large amounts of current news from a range of sources and perspectives, autistic individuals could gain a greater understanding of the social and political context of their advocacy movement (Kras, 2009).

Advocates created and organized other important websites, sources of information, and Autistic-specific communities through the late 1990s to today. Wrong Planet, one popular Autistic website, was started in 2005. It includes a chat room, forum, dating, and articles for autistic individuals, and has grown to over 60,000 registered members since its beginning. Aspies for Freedom, an Autistic self-advocacy organization established in 2004 , created a website with similar various modes of connecting with others and the community online. Aspies for Freedom also gained attention in the online Autistic community for owning an island in Second Life for Autistic individuals that even contained an autism museum. With the growing number of sources of information, advocates created Autism Hub to pull together many of the blogs from self-advocates, parents, and professionals on autism. This resource also became an important source of information for Autistic individuals.

The Autistic community emerged through the convergence of growing awareness and diagnosis of autism (Grinker, 2007), the developments of the disability 
rights movement, as well as the growth and accessibility of computer technology and the Internet (Bagatell, 2010; Blume, 1997b). As with many successful self-advocacy movements seeking equality through grassroots organizing and social action (Chamak, 2008; Klawiter, 2012), as the Autistic self-advocacy movement grew, it faced opposition from individuals outside their community as well as experienced internal tensions. As ANI encountered, tensions exist around how to involve non-autistic individuals interested in being a part of the community due to their profession or autistic family member. Beyond having different interests and motivations for involvement in the community, many non-autistic parents and professionals hold fundamentally different views of the representations of autism, appropriate treatments for autistic individuals, and the need for a cure for autism (Singer, 1999). The majority of individuals that advocate for a cure or prevention of autism is non-autistic parents and professionals who view autism as prohibiting autistic children from living their lives to the fullest. They also argue that an autistic child creates a great burden on the family and social network of the child that should be eliminated if autism was cured. The large majority of organizations run by non-autistic persons emphasize a cure, as well as research striving for a cure (e.g., National Institute for Child Health and Human Development, Autism Speaks). Similar tensions exist with the discussion regarding treatment. Non-autistic parents and professionals advocate for treatments such as applied behavior analysis, in which autistic individuals are trained in "normalizing" their behaviors and reducing their autistic traits (Jensen, 2002; Tager-Flusberg, Paul, \& Lord, 2005). Many Autistic self-advocates hold a competing view, saying such "cures" and 
intrusive treatments do not make actual changes to the person, just cover up any characteristics others see as undesirable, which may lead to suffering in the child (Dawson, 2004; Ne'eman, 2009). Further, prevention of autism may lead to eugenic abortion as seen in the Down syndrome community (Ne'eman \& Asch, 2010). The common argument against Autistic self-advocates' anti-cure stance, echoing critics of self-advocacy organizations, is that only those considered "high-functioning," or not autistic are anti-cure, and do not take into consideration the greater issues faced by "low-functioning" individuals.

Notably, exceptions exist to the dichotomy of parents and professionals supporting a cure versus self-advocates rejecting a cure (Ortega, 2009a). Some parents have spoken out against a cure for autism (Gernsbacher, 2004) and some Autistic selfadvocates have supported a cure. Historically, tensions and dissent in social movements are necessary for strengthening the movement (Chamak, 2008). Amanda Baggs, an Autistic self-advocate and member of AASPIRE, wrote: “Communities aren't about liking each other, they're about bothering to do things for and/or with each other even if you don't like them. Likewise advocacy involves sometimes gritting your teeth and doing things alongside people you don't like, rather than sitting there grumbling about why you don't like them (and by "don't like" I'm talking personality conflicts here). Even if they're grumbling pettily about you." (Baggs, 2007; para. 13). As the online Autistic community reacts to the commonly held views on cures and treatments, ideas and individuals clash, strengthening political and social consciousness. Within the anti-cure 
and anti-invasive treatment self-advocacy community, a further emphasis exists on the value of diversity rather than acceptance or a lack of rejection.

Formation of the Autistic community resulted in and grew through major events and milestones that have strengthened the community and increased society's awareness and positive views of the Autistic community. Through this growth, Autistic individuals relied heavily on the Internet to connect with one another, inform members, and perform actions. Online activities of Autistic self-advocates have served to further cement an Autistic culture online and offline. Next I will discuss some of the major events and milestones of the Autistic community.

In reaction to the lack of an Autistic presence at autism conferences, ANI hosted the first Autreat Conference in 1996, which is still held annually. This conference and retreat is run by and for Autistic individuals. It focuses on positive living for autistic individuals and developed to be a good environment for autistic adults, particularly around sensory practices and choice to participate and community with others at the conference. Similarly, Autscape started in 2005 in the United Kingdom. It serves as a similarly self-advocate run conference and retreat for individuals in the UK.

The year 2005 also marks the first celebration of Autistic Pride Day, started by the organization Aspies for Freedom, with the goal of promoting acceptance and pride in the Autistic community, much like gay pride celebrations. Autism Acceptance Day was proposed by Paula Durbin-Westby, an Autistic self-advocate and member of AASPIRE, in 2010 in reaction to Autism Awareness Day. World Autism Awareness Day, April $2^{\text {nd }}$, was declared by the United Nations as a day to promote awareness of autism as a disease, 
with events for supporting cure and treatment worldwide. Autism Speaks, the largest autism organization in the world, is a non-autistic parent and professional organization that has given World Autism Awareness Day international recognition and organized large-scale fundraising efforts focused on funding research aimed at a cure for autism and casting a deficit-based view of autism. Conversely, Autism Acceptance Day is promoted by Autistic self-advocacy organization, and celebrates diversity and a strengths-based view of autism. Local advocacy groups worldwide have caught onto the reactive holiday, and expanded it first to naming April Autism Acceptance month, then Autism Acceptance Year running from April 2012 to April 2013.

In addition to Autistic self-advocates organizing conferences and promoting holidays, political actions served as important milestones for the Autistic community, such as the "Ransom Notes Affair" (Kras, 2009). The NYU Child Study Center promoted their work through billboards constructed in New York City in 2007 that compared autism to a kidnapper snatching children from society. Ari Ne'eman, an Autistic selfadvocate, lead an online petition and letting writing protest campaign against the NYU Child Study Center with Autistic advocates and other disability rights organizations on the basis that the ads were stigmatizing, that they inaccurately depict disabilities as only negative, and that they give a message to parents that their children are doomed to suffer due to their disability. The NYU Child Study Center took down the billboards and presented an apology for the message. Ari Ne'eman founded the Autistic Self-Advocacy Network (ASAN) in 2006 with Scott Robertson, but after the successful campaign, local chapters were formed and expanded across the US, Canada, and Australia. The ransom 
notes incident is also said to have bolstered the Autistic community as a whole, with a strong victory resulting with their unification and energetic involvement (Kras, 2009).

Ne'eman and Robertson formed ASAN as a community-based organization that seeks to advance the Autistic self-advocacy movement through organizing the community to have their voices heard in order to promote the same access, rights, and opportunities as other citizens. One focus of ASAN includes the funding and topics of autism research. All federal funding for autism research is coordinated with the Interagency Autism Coordinating Committee (IACC) within the Department of Health and Human Services (HHS). The IACC compromises non-autistic professionals with a deficit-focus and goal of "combating autism" through treatment and cure. Further, nonautistic professional and parent organizations such as Autism Speaks have largely been the other major funders of autism research. This leads to a small amount of autism research focusing on strengths-based research with autistic adults (IACC, 2012). Due to this gap in research, AASPIRE was formed in collaboration with ASAN to study issues important to the Autistic community.

After the formation and international expansion of ASAN, Ari Ne'eman gained recognition as a leader in the Autistic self-advocacy movement. In 2009, President Obama appointed Ari Ne'eman to serve on the National Council of Disability, making Ne'eman the first openly Autistic appointee. Ne'eman's role not only serves to give an Autistic voice to national issues, but also a symbol of the importance of self-advocacy roles in decision making and further visibility of the Autistic community in society. 
In addition to the positive milestones in the Autistic self-advocacy movement, periods of disharmony have occurred that are not uncommon in any community. Particularly relevant to the current study, the online Autistic community experienced turmoil in a time period that overlapped with data collection, from 2010 to 2012. In 2010, a number of websites were sources of cyber bullying from a small but impactful minority of online Autistic community members. This small group of cyber bullies focused comments and blog entries on attacking individuals in a number of ways, conveying misogynistic or racist attitudes, or calling into question whether or not others in the community were autistic (Ditz, 2010; http://lizditz.typepad.com/). Due to the negativity amongst community members, organizations and individuals requested removal from the popular site AutismHub, a website that hosted a large number of autism-related blogs which served as a centralized location for the online Autistic community. Eventually, the founder of AutismHub was forced to shut down the site (Leitch, 2010; http://leftbrainrightbrain.co.uk/2010/05/07/the-autism-hub/). During this time, a number of Autistic websites became combative, resulting in increased cyber bullying and removal of comments or blog posts, as well as a number of popular bloggers deciding to stop blogging altogether. Individuals decided to leave the online Autistic community because they felt unsafe and unhappy within the community. While the disharmony may have impact how individuals perceive the community, the online Autistic community has since recovered from the turmoil, with some individuals who previously left the community returning, and many websites becoming active once again. AutismHub was eventually active again, though now under new, anonymous 
administrators rather than the founder and original administer (http://autismhub.com/?page $i d=2$ ). As bloggers have commented on the negative experience in the community, the turmoil mirrors cyber bullying and within-group struggles that are also common in non-autistic online communities (Ditz, 2010).

The history of the disability rights movement, the Autistic self-advocacy movement, and the online Autistic community led to a re-definition and recharacterization of autism from the original, broader societal view of autism that I described earlier. Next, I talk about what the definition and characteristics of autism are within the context of the Autistic self-advocacy movement, as therefore how autism is framed in this study.

\section{Re-Defining and Characterizing Autism within the Self-Advocacy Movement}

While the conceptualization of autism within the self-advocacy movement is not completely separate from the traditional medical conceptualization, there are unique emphases that are common within the diverse perspectives of the self-advocacy movement. Autistic advocates tend to be critical of definitions of autism by non-autistic professionals in the field of autism research, rejecting the pervasive influence of these definitions on the media, in power over resources, and public mindshare (Clarke \& Van Amerom, 2007; Yergeau, 2010). Instead such individuals identify multiple subjective understandings of autism through the perspective of each autistic individual (Biklen, 2005). Most autobiographical accounts by autistic individuals focus on the unique strengths and challenges, perspectives, and knowledge that come with autism rather 
than considering autism a deficit (Davidson, 2008; Happé \& Ronald, 2008; Wing, Gould, \& Gillberg, 2011).

In addition to a focus on unique strengths and challenges, Autistic individuals who identify with the self-advocacy and neurodiversity movements often reject notions of a cure for ASD because the disorder is a pervasive aspect of every autistic person's life that should not and likely cannot be taken away with a cure (Sinclair, 1998). Unlike many people with disabilities, many Autistic self-advocates reject "person first" language (i.e., person with autism) and prefer identity first language (i.e., autistic person) when referring to being on the spectrum, presenting autism as an integral aspect of their being, not simply an add-on to oneself (Bagatell, 2010; O'Neil, 2008; Orsini \& Smilth, 2010; Ortega, 2009b; Silverman, 2008). While Autistic individuals recognize the challenges of being on the spectrum, including unique support needs, these individuals feel that the majority of the difficulties experienced by autistic individuals are rooted in the pressures of society to conform to typical social norms (Boundy, 2008). Supporters of the self-advocacy movement are also in favor of the individuals having the knowledge and choice of the use of therapies, treatments, and medications in their lives (Boundy, 2008).

Additionally, Autistic self-advocates emphasize that autism spectrum disorders are not on a continuum of mild to severe forms of autism; because disabilities are a function of the context, individuals deemed "severely autistic" are in a severely unaccommodating environment (Biklen, 2005). Self-advocates consider the binary or spectrum of functionality as a medical construct that uses neurotypicality as a standard, 
although the concept of neurotypicality is undefined, unstable, and contextually dependent (Yergeau, 2010). Furthermore, as each autistic individual embodies unique characteristics related to and not related to autism, a continuum does not account for the unevenness of functioning within an environment or across various environments. Unevenness of functioning is also observed across lifespan, with individuals viewed as "high" or "low functioning" over extended periods of their lives. Static diagnoses or labels do not consider such changes. Lastly, even within specific supportive environments, too much individual variation exists among autistic individuals to say what challenges are universal within specific diagnoses. Educators, practitioners, and researchers who adopt this perspective of the Autistic community tend to emphasize the strengths of autistic individuals and recalibrate the focus of their work to see the autistic person as an individual with gifts and talents as well as challenges, emphasizing the need to conduct research directly with autistic individuals to capture their perspectives rather than outsider perspectives of autistic individuals (Robertson, 2010). This approach is in contrast to much traditional research that relies on proxy report to represent the voices of autistic individuals (Shipman, Sheldrick, \& Perrin, 2011).

In addition to the Autistic self-advocacy movement impacting the way autism is defined and characterized, the movement has had an impact of how society views Autistic self-advocates as a group. Jim Sinclair and the other co-founding members of ANI named their organization a "network" because a community seemed infeasible (Sinclair, 2005). However, as time passed and they found that networking resources, information, and stories resulted in more than an organization or a series of friendships. 
A community grew larger than the individual organization or any other organizations alone. The connections that Autistic individuals made through the Internet changed how individuals viewed autism. Despite stereotypes and diagnostic criteria of autism, or how improbable in may have seemed in the early 1990 s to the co-founders of ANI, the Autistic self-advocacy movement became a community. To formally reflect this acknowledgment of a community and culture existing around autism, advocates began capitalizing the " $\mathrm{A}$ " in Autistic community or Autistic culture. This change mirrors the " $D$ " in deaf, which is capitalized when referring to the Deaf community or Deaf culture but not when used as a plain adjective (i.e., a deaf individual within the Deaf community). Advocates began promoting the usage of "Autistic community" versus "autistic community" in the early 2010s, and the Autistic community has widely adapted and promoted the terminology (Ashkenazy, personal communication, May $17^{\text {th }}, 2011$ ).

These defining aspects of the new characterization of the Autistic community lead to the creation of a cohesive community identity. Like the broader disability rights movement, the community used their defining characteristics towards identity politics, where the formerly stigmatizing aspects of their identity were transformed into sources of pride and arguments for social inclusion and self-advocacy (Siebers, 2006). As the Autistic self-advocacy movement gains momentum, their identity politics serve as an important source of unity and strength.

Throughout the development of an Autistic community, the Internet plays an important role. The Internet allows individuals to meaningfully connect with one another and share one's experiences and read others' experiences with the strengths 
and challenges of being autistic. The Internet also allows members of the Autistic community to develop political consciousness and education, and to organize and advocate for rights and needs. The next section defines community and community involvement, and then discusses involvement in the online Autistic community and how involvement in the online Autistic community may be related to the positive outcomes of Autistic identity and self-determination in autistic adults. 
CHAPTER III

Review of the Literature

The historical context of the disability rights movement and the Autistic selfadvocacy movement provide a rich background for a better understanding of the online Autistic community. To contribute and promote the community, researchers must empirically examine positive outcomes of involvement in the online Autistic community, such as Autistic identity and self-determination, as well as whether communication preference may moderate such relationships in autistic individuals and non-autistic individuals. This chapter's review of the literature describes the empirical and theoretical basis for this study's concepts of interest. I discuss involvement in the online Autistic community, communication preferences, Autistic identity, and selfdetermination in this chapter.

\section{Involvement in the Online Autistic Community}

Sarason (1974) defines community as "a readily available, mutually supportive network of relationships on which one could depend" (p.1). Communities are often dichotomized into two types of communities. Communities of locality or geographic communities include blocks, neighborhoods and cities. Relational communities or communities of interest are support groups, church congregations, or Internet groups. Some authors report an alarming decrease in community involvement and sense of community (Dalton, Elias, \& Wandersman, 2000; Putnam \& Miller, 1995). However, 
these reports do not consider the impact of technology and our shifting, global world, where others have cited the Internet as a new, exciting tool to promote sense of community (Obst, Zinkiewicz, \& Smith, 2002a). The Autistic community is such a community that developed because of the Internet.

The online Autistic community is a relational community of individuals who have found communalities, support, and friendship based on interest in the Autistic selfadvocacy movement or seeking individuals' experiences with being autistic. As described in Chapter II, the existence of an Autistic self-advocacy movement may be dependent on involvement in the online Autistic community. The unique historical underpinnings of this community suggest a need to empirically examine the impact of the online Autistic community on autistic individuals.

The Internet is a source of information for and about autistic individuals. Autismfocused websites and list serves provide the opportunity for sharing first-hand accounts and perspectives and for providing more complex and accurate portrayals of the emotional and social lives of autistic people (Jones, Zahl, \& Huws, 2001; Jordan, 2010). Promoting the voices of these individuals among the disability community and beyond created a powerful movement that is reflected in all aspects of Autistic individuals' lives (Blume, 1997b) as well as how autism is perceived by mainstream society (Davidson, 2008).

The Internet also allows individuals to communicate and foster relationships over geographic distances using text-based communication, which is preferred by some autistic individuals (Benford \& Standen, 2009). The Internet is also valuable to the 
Autistic community beyond social interaction. Folstein (1999) argues that differences in social abilities in autistic adolescents and adults can account for under-employment and difficulty in relationships. Autistic adults are not often given the tools or opportunities to access to information, people, or services that can assist them in making critical life decisions (Barnard, Harvey, Potter, \& Prior, 2001). The Internet allows individuals to learn from one another as well as work together for advocacy purposes. Additionally, since many of these websites are free and have open access, they create an opportunity for others not on the spectrum to learn and understand more about the nature of autism.

The online Autistic community may be a unique population of individuals; however, this community is not studied enough to draw conclusions about demographics or diagnoses. Due to the lack of research on this population, many call for a greater understanding of the personal experiences of autism (Barrett, 2006) and the way autistic people are part of communities (Silverman, 2008). Since researchers are unsure of exactly who participates in the online Autistic community, readers should be cautious about generalizing findings from the online Autistic community to all individuals, or all autistic individuals (Jones et al., 2001).

Among the general population, Internet use has dramatically increased in all age groups in the past ten years (Zickuhr \& Smith, 2012). It was formerly accepted that age disparities existed among individuals with disabilities and autistic individuals that use the Internet (Cromby \& Standon, 1999). Since recent research on Internet use reflects similarities between autistic adults and the general population (Mazurek, 2013), the 
only age disparity that currently exists is in adults over the age of 65 , with only $41 \%$ of this age group reporting any Internet usage as compared to $74 \%$ to $94 \%$ in younger age groups (Zickuhr \& Smith, 2012). However, due to autism diagnostic criteria changes occurring in the 1980's and 1990's, older adults may not have a diagnosis or identify as ASD or have exposure to the ideas of the Autistic self-advocacy movement (Schur, Shields, \& Schriner, 2005), thus limiting involvement in the online Autistic community. Age is examined in the current study by determining whether age differences exist among key variables in each analysis.

In addition to age, research examines income in relation to Internet use, with greater number of individuals reporting Internet use as income increases (Zickuhr \& Smith, 2012). Of individuals with income at or below $\$ 30,000$ a year, $62 \%$ report Internet use as compared to the $83 \%$ to $97 \%$ in higher income brackets. The online Autistic community may be mostly populated with people above a certain income (Cromby \& Standon, 1999). However, disability and poverty are closely associated (Erickson \& Lee, 2008), which may partially explain why 54\% of adults with disabilities use the Internet as compared to the $81 \%$ of individuals with no disabilities that report using the Internet (Zickuhr \& Smith, 2012). This income disparity indicates a need to understand how income may be related to the online Autistic community. Therefore, income is examined in the current study as well to determine whether differences exist between groups with difference communication preferences or between autistic and non-autistic participants. 
While age and income disparities provide important information, demographic indicators of who may be involved in the online Autistic community do not offer a complete understanding of the composition of the online Autistic community. A greater understanding of who is involved in the online Autistic community and what constitutes involvement in necessary for empirically investigating the relationships between involvement in the online Autistic community and positive outcomes of identity and self-determination. Qualitative studies investigate Autistic culture both online (Davidson, 2008) and more generally (Silverman, 2008). Additionally, personal accounts of how the Internet has impacted the lives of autistic individuals are readily available on many blogs, videos, chat rooms, and list serves. Research also investigates how identity plays a major role in this community, finding that Autistic identity and identification with the Autistic community is a complex, dynamic construction for every individual involved in the online Autistic community (Brownlow, 2007a). Further, my thesis study investigates individuals' perceptions of the importance of involvement in the online Autistic community and its relationship to identity, sense of community, and well-being (Kidney, 2012).While the thesis study supported positive relationships between importance of involvement in the online Autistic community and Autistic identity as well as sense of community, a logical next study should consider a broader conceptualization of involvement in the online Autistic community. By utilizing a theoretically and statistically sound measurement, this study sheds light on how aspects of involvement may uniquely contribute to involvement in the online Autistic community. Here I discuss 
how involvement in the online Autistic community is conceptualized and measured in the current study.

Internet use and online socializing are related to increased social capital, social engagement, and relationship closeness (Boase et al., 2006; Gross et al., 2002; Katz et al., 2004; Reich et al., 2012). That is, the Internet allows for individuals to seek help for their problems, and facilitate regular contact with a larger number of individuals both nearby and far away (Boase et al., 2006). Online networks span geographical locations and allow people to grow number of connections while not having negative impact of traditional bases of communities such as relatives, friends, and co-workers (Boase et al., 2006; Reich et al., 2012). Further, a connectedness exists among social network modes, where more online communication is related to more face-to-face and phone communication. The study shows online communication does not replace other modes of communication, but does replace time otherwise spent sleeping or watching TV. Social capital in turn impacts quality of life and well-being (Kraut et al., 2002; Putnam \& Miller, 1995). Additional research suggests the positive relationship between Internet use and well-being (Kraut et al., 2002; McKenna \& Bargh, 1998).

However, contrasting evidence indicates that Internet use does not impact on offline social networks (Pollet, Roberts, \& Dunbar, 2011) or that Internet use negatively impacts well-being, particularly for individuals who excessively use the Internet in an attempt to alleviate loneliness caused by a lack of face-to-face social skills (Caplan, 2005; Kim, LaRose, \& Peng, 2009). Literature defines “problematic Internet use" as a maladaptive preoccupation with the Internet that results in negative social, academic, 
or professional consequences due to prolonged periods of time online (Caplan, 2002;

2005; Davis, 2001; Shapira et al., 2000). While research links problematic Internet use to negative outcomes in a person's life (Caplan, 2003), important mediators in the relationship may exist such as the ways in which the Internet is used (e.g., for gaming versus email) and the presence of underlying characteristics such as depression or social anxiety (Bell, 2009; Shaffer et al., 2000). The problematic Internet use literature also tends to cast traditional face-to-face communications as the superior mode of socializing with preference for online communication considered a risk factor for problematic Internet use and, in turn, negative outcomes such as depression and loneliness (Caplan, 2003, 2010). One study found a positive relationship between Internet addiction and autistic traits (as measured by the Autistic Spectrum Quotient; Baron-Cohen, Wheelwright, Skinner, Martin, \& Clubley, 2001), as well as depression, anxiety, and schizotypal traits (Romano, Osborne, Truzoli, \& Reed, 2013). While findings imply that extended Internet use may have a negative impact on individuals, the evidence was correlational and did not consider potential relationships with positive constructs such as social support or identity development. This evidence suggests a need to further understand the impact of Internet use on autistic individuals, and whether other factors such as communication preference influences relationships. The unique communication and socialization preferences and needs of autistic individuals may create circumstances in which problematic Internet use should be reframed. That is, if a person thrives with and is personally/socially rewarded with online 
communication while struggling with communication offline, perhaps face-to-face interactions should not be considered superior.

Also contrary to research pointing out the negative impact of Internet use, researchers explore the use of the Internet by persons with disabilities, which serves as a tool for empowerment and social interaction (Brownlow \& O’Dell, 2002; Seymour \& Lupton, 2004; Siddiquee \& Kagan, 2006). Many people with disabilities use the Internet in the same way as people without disabilities, such as for information finding, personal communications, and shopping (Burks et al., 2000). A study compares deaf adolescents to hearing adolescents in Internet use, findings that the deaf group used the Internet more than the hearing participants (Barak \& Sadovski, 2008).

A qualitative study suggests that Internet use for socializing and involvement in the online Autistic community has a positive impact on initiating relationships, but is also related to difficulties in maintaining relationships or trusting strangers online (Burke et al., 2010). Other qualitative studies indicate that the online Autistic community is central to an autistic person's conceptualization of self, and that the online community is one of few spaces considered safe for interacting with others (Brownlow, 2007a; Davidson \& Henderson, 2010; Davidson, 2008; S. Ryan \& Räisänen, 2008). However, there is no quantitative research that examines positive outcomes of involvement in the online Autistic community beyond analysis of preliminary data in the current study (Kidney, 2012). This study seeks to understand the components of involvement in the online Autistic community, and examine how involvement in the online Autistic community is related to Autistic identity and self-determination. Further, this study 
compares the strength of communication preference moderating involvement in the online community and self-determination between autistic and non-autistic adults.

Involvement in online communities is conceptualized and measured in a variety of ways in the literature, from examining hours spent online (Kraut, Patterson, \& Lundmark, 1998), types of online activities (Gallup, 2009; Kim, Kim, Park, \& Rice, 2007), and coding qualitative data for social interactions (Burke et al., 2010). Inconsistencies in conceptualization and measurement yield incomplete empirical information about involvement in the online Autistic community and involvement in any online community for non-autistic individuals. No study has examined involvement in the online Autistic community as a distinct, multi-dimensional concept. Therefore, this study developed a multidimensional measure of involvement in the online community taking a data-driven and theoretically sound approach (Njong \& Ningaye, 2008). The dimensions of involvement in the online community that were measured in this study include frequency of involvement, years of involvement, importance of involvement, and sense of community. For the measurement of involvement in the online Autistic community, frequency, years, importance, and sense of community are measured by items and a scale that refer specifically to the online Autistic community. For the measurement of involvement in the online community in non-autistic adults, frequency, years, and importance items ask for involvement on "any sort of online community," and the scale instructions for sense of community refer to "the aspects of the online community in which you feel most involved." Next I discuss the specific constructs and measurements of the domains of involvement in the online Autistic community and involvement in the 
online community for non-autistic adults, as well as rationale for the use of the constructs in the current study.

\section{Frequency of involvement in online communities.}

As Internet access and widespread use increased, concerns arose about the potential negative consequences of spending time online (Kraut et al., 1998). Researchers found that frequency of Internet use, measured as hours spent online per week, is negatively related to well-being and social involvement (Kraut et al., 1998). The same sample was surveyed again three years later, adding a control group and redefining social involvement to include both local and distant social circles and broader community involvement; Kraut and colleagues found the negative outcomes on wellbeing and social involvement dissipated (Kraut et al., 2002). Recent literature focuses on debunking concerns that other aspects of a person's life will suffer due to the amount of time spent online (Howard, Rainie, \& Jones, 2001; Rohall \& Cotten, 2002; Valkenburg \& Peter, 2007; Wellman \& Gulia, 1999). When studies consider quality of life issues longitudinally, use control groups, and consider the new, potentially geographically disparate and interest-focused qualities of social connections, the amount of time spent online is positively related to social involvement (time spent communicating with family, size of local social network, size of distant social network, perceived social support), easier interactions, less time watching TV, and more involvement in the local community (Boase et al., 2006; Kraut et al., 2002; Valkenburg \& Peter, 2007). Therefore, online socializing may be responsible for an increase in social involvement and potential 
benefits to quality of life when traditional views are re-defined to consider that social support, quality relationships, and community involvement may be fostered and maintained online.

Research in neurotypical samples often lacks the important considerations for the online Autistic community. Whereas neurotypical social circles often exist in geographically local communities that may be strengthened by increased online socializing, autistic individuals may not have the face-to-face social support before seeking relationships online (Sinclair, 2005). The small number of geographically dispersed individuals in the Autistic community may not have many opportunities outside of online interactions to connect with one another, like in other geographically dispersed marginalized communities (McKenna \& Bargh, 1998). Therefore, the amount of time spent interacting with others in the online Autistic community may compromise the majority or entirety of social interactions for autistic individuals in a peer-support setting. The current study is the first quantitative study to examine the relationship between frequency of involvement in the online Autistic community and positive outcomes, whereas frequency of involvement has only been utilized in studies with neurotypical populations (Kraut et al., 2002). This study utilizes frequency as a component of the involvement in online Autistic community measurement as well as for the involvement in the online community for non-autistic individuals.

Despite the widely utilized measure of number of hours of Internet use as a predictor of social and quality of life outcomes (Howard et al., 2001; Nie \& Erbrin, 2000), the measure disregards important aspects of community involvement. New Internet 
users may use these hours to acclimate to and explore the Internet rather than engaging in quality interactions and developing a socially supportive community, and many numbers of hours spent online may decline or end abruptly. Additionally, the emotional connection to the community or engagement with the community is not captured with number of hours. Therefore, this study conceptualizes and measures involvement in the online community for both autistic and non-autistic individuals as compromising the additional domains of years of involvement, sense of community and importance of involvement in the online community.

Years of involvement in online communities. Internet use across all ages, cultures, income levels, and education levels is steady increasing since the early 1990s (Gallup, 2009). The increase in Internet access means more individuals are online for a greater number of years. Gaining years of experience in using the Internet means that individuals possess increased Internet skills, greater comfort and trust in the Internet, and perform higher number of activities online (Gallup, 2009; Nie \& Erbrin, 2000). Since the majority of time spent online is for social reasons, particularly email (Nie \& Erbrin, 2000), an increase of Internet use may mean an increase in online socialization, and an exploration of alternative ways to connect with one another (Kraut et al., 2002). For autistic individuals, this may mean they spend time exploring autistic websites, or view or contribute to websites that deepen connections to the Autistic community (Hand, 2011). Further, since the online Autistic community is a relatively new community that gained momentum through the mid to late 1990s, members involved since the 
beginning years of the community likely have a deep connection to the community that would relate to higher Autistic identity and self-determination. However, years of involvement in any online community has not been studied in-depth in relation to any social or quality of life variables. This study sought to add to the literature by examining how years on involvement in a community may impact identity and self-determination.

By examining the impact of years of involvement in tandem with frequency of involvement, this study measured a slightly more complete picture of involvement in the online community for autistic individuals and non-autistic individuals. However, the measurement of involvement in the online community must also consider subjective indicators of the quality of involvement, lest the years spent involved in the online Autistic community and the frequency of involvement yielded unsuccessful social ties or negative experiences (Burke et al., 2010) that would likely not relate positively to Autistic identity or self-determination. The two subjective indicators that serve as measures of the quality of involvement in the online Autistic community are described next as sense of community and importance of involvement in the online Autistic community.

Sense of community in online communities. The literature indicates that quality of the interactions individuals have within their community is an important contributor to positive social and quality of life outcomes (Burke et al., 2010; Kraut et al., 2002; Wellman \& Gulia, 1999). Sarason (1974) defines psychological sense of community (SOC) as "the perception of similarity to others, an acknowledged interdependence with 
others, a willingness to maintain this interdependence by giving to or doing for others what one expects from them, the feeling that one is part of a larger dependable and stable structure" (p. 157). Empirical work shows that SOC is closely related to community involvement and action in neurotypical communities (Allen \& Allen, 1987; Bachrach, Kenneth \& Zautra, 1985). Further, a significant positive relationship between the importance of involvement in the online Autistic community and SOC in the online Autistic community exists in a subset of the study sample (Kidney, 2012). The theory of SOC lends itself to a proxy measure of the subjective quality of community involvement due to the empirical relatedness to community involvement as well as SOC's theoretical basis as developed by McMillan and Chavis (1986). McMillan and Chavis' (1986) theoretical model of SOC contains four dimensions (membership, influence, integration and fulfillment of needs, and shared emotional connection). Much of the research investigating SOC uses and supports the model, including in online communities (Obst, Zinkiewicz, et al., 2002a). This study utilized three of the most relevant dimensions for the SOC component of involvement in the online Autistic community and online community, including membership, influence, and integration and fulfillment of needs. The study utilized the SOC dimension of shared emotional connection as a measure of the relatedness dimension of self-determination, which I describe in the SelfDetermination section later in this chapter. The conceptual connection to the selfdetermination subscale lends itself more closely to the dimension of shared emotional connection than to involvement in the online community. The remaining three 
dimensions are concepts that relate to specific aspects of community involvement, which I describe here.

McMillan (1996) defines membership as a sense of belonging or feeling a part of the community. Membership provides emotional safety and includes having a personal investment in the community, which lead to stronger bonds (McMillan). Defined boundaries for the community and feeling as though one is within them are also important in this concept. In the case of Internet communities, communities can define boundaries as loosely as subscribing to a listserv or as strict as requiring an invitation to join a message board (Forster, 2004). These boundaries may play a part in the strength of feeling of membership individuals have with their community (McMillan \& Chavis, 1986). Feeling a sense of membership is likely an important indicator of a subjective judgment about being involved in the community; without membership in the community, a person would not feel involved in the community.

McMillan (1996) defines the dimension of influence as having a sense of control over the community. It is a bidirectional concept with the community having control over the individual as well (McMillan \& Chavis, 1986). The idea of influence is related to group processes such as conformity and the tensions of leader and follower dynamics (McMillan). This dimension also captures the cohesiveness and development of community norms. In Internet communities, this may involve respect and trust in message board moderators and the feelings that accompany heavy moderation, such as approval for messages sent out to the community. Additionally, more homogeneous and conforming messages and emails to the community may indicate the influence of 
the community on the individuals participating, pressures of conformity, and community norms leading to order. Here, active participation and control on the community may indicate higher involvement in the community, as well as community influence on the individual. Such transactional interactions among an individual and the community are likely necessary for the individual to feel involvement in the community.

The concept of integration and fulfillment of needs involves an individual's perception of the rewards of being a member of a community (McMillan \& Chavis, 1986). Because of similarities among members and the support provided to one another, individuals perceive fulfillment from being a part of a community. If members see the community as successful with competent members cooperating and helping one another out, they are likely to feel that their needs are fulfilled as expected from the community. This dimension of integration and fulfillment of needs encompasses the emotional rewards from a sense of togetherness. In Internet communities, this may involve helpful and engaging discussion and a positive sense of accomplishing goals as a community. The concept of integration and fulfillment of needs is likely a strong indicator of the quality of involvement in the community. Beyond time spent with the community or even feeling a sense of measurement, if individuals feel as though their needs are met through time spent with the community, they would feel as though the time spent with the community is of better quality. A more complete concept of involvement in the community is created by having a multidimensional measure of involvement in the online community that encompasses such indicators of subject quality of involvement. Further, a sense of integration into a community implies that 
individuals are involved in a community to an extent that they share an identity and important similarities with the community, thus indicating a high quality of involvement in the community.

The theoretical model for sense of community has been accepted and broadly used in sense of community research (Chipuer \& Pretty, 1999). However, the measurement of sense of community is scrutinized and often modified since its original form. Chavis, Hogge, McMillan, and Wandersman (1986) developed the Sense of Community Index (SCI), a 46-item scale to measure psychological sense of community according to the four theoretical dimensions. As researchers may apply the theoretical model to any locality or relational community, the $\mathrm{SCl}$ can be used to measure $\mathrm{SOC}$ in any community (Chavis et al., 1986). Chavis and colleagues (1986) created a 12-item index using a Brunswik's lens methodology, a process of developing items through consensus of the experience of sense of community and prediction of sense of community by the items and language agreed upon (Chavis et al., 1986). This index became the most widely used measurement of SOC in community psychology that was adapted for a wide variety of communities including the Internet (Forster, 2004; Obst, Zinkiewicz, et al., 2002a; Obst, Zinkiewicz, \& Smith, 2002b). The index is also the only measurement developed from the sound McMillan and Chavis model (Obst \& White, 2004). Despite critiques of the scale that a fifth dimension may exist, the scale does not reliably perform across populations, and that the scale is unidimensional (Chipuer \& Pretty, 1999; Obst, Smith, \& Zinkiewicz, 2002; Obst \& White, 2004), researchers widely accept and use the scale. Since community members sense similarities with one another 
and have a strong sense of a unique culture and community within the online Autistic community (Silverman, 2008), McMillan and Chavis's theory is suitable to use as a dimension of involvement in the online Autistic community. Past research applied this theory to relational communities online (Forster, 2004). Further, the three subscales of the $\mathrm{SCl}-2$ utilized for the current study are significantly related to the importance and value of the online Autistic community in a subset of the current study sample (Kidney, 2012).

Chavis, Lee, \& Acosta (2008) improved the measurement of sense of community. The latest measurement development on the $\mathrm{SCl}$ involved creating a 24 item scale based on the original 12 item scale, but utilizing a Likert scale rather than the original true-false format (Chavis, Lee, \& Acosta, 2008). This scale, the $\mathrm{SCl}-2$ was piloted, revised, and tested with a large population. Previous studies as well as the current study sample indicate high internal consistency with overall alphas of .91 and .94 in the autistic and non-autistic samples respectively.

With the addition of sense of community to the multidimensional measure of involvement in the online Autistic community, the measurement includes a subjective, quality-based measurement of involvement with the frequency and years of involvement in the online Autistic community. The final dimension of the measure is described next, which adds another subjective measure of the quality of involvementimportance of involvement in the online Autistic community. 
Importance of involvement in online communities. Measuring the importance

of involvement in the online Autistic community adds a value-driven, subjective assessment of involvement. As described in the previous section on sense of community, importance of involvement in the online Autistic community is positively correlated with SOC and each of the SOC subscales (Kidney, 2012). However, the importance of involvement may tap into an aspect of community involvement beyond strong social ties and a sense of community. Importance implies a sense of necessity and desire to create positive change and advocate for oneself and one's community. This idea relates to Amanda Bagg's musings on involvement in the online Autistic community, where she states, “Communities aren't about liking each other, they're about bothering to do things for and/or with each other even if you don't like them. Likewise advocacy involves sometimes gritting your teeth and doing things alongside people you don't like, rather than sitting there grumbling about why you don't like them..." (Baggs, 2007, para. 13). A community's strength may be in both its cohesion among members as well as the diversity and differences each individual brings to it (Townley, Kloos, Green, \& Franco, 2011).

Further, the AASPIRE team agreed with Baggs' sentiment while providing input regarding measurement of involvement in the online Autistic community in my thesis study. Community partners believed that the importance of involvement may be a more accurate measurement of involvement in the online Autistic community than frequency or years of involvement, since it indicates that commitment and value placed on 
involvement beyond potentially insignificant hours or years of time spent with the community. Therefore, this dimension of involvement in the online Autistic community was included to reflect the community's perspective and complete the multidimensional, composite indicator.

In summary, involvement in the online Autistic community has not been quantitatively examined beyond importance of involvement in the online Autistic community (Kidney, 2012). This study developed a multidimensional indicator of involvement in the online community that taps into both more objective quantities of involvement (frequency of involvement and years of involvement) as well as subjective, quality-driven domains of involvement (sense of community and importance of involvement). As the current study is the first of its kind to develop a multidimensional measure of involvement in the online community for autistic individuals and nonautistic individuals, the dimensions I chose for inclusion were based on previous research, theory, and community input. This study took these dimensions and develops an empirically supported data-driven weighting system to determine how the variable of involvement in the online community should incorporate each of the dimensions (Njong \& Ningaye, 2008). The method of using principle components analysis to assign weights to each dimension is described in detail in the Analysis section.

In addition to understanding the multidimensional concept of involvement in the online community for autistic and non-autistic individuals and how it is related positive outcomes such as Autistic identity and self-determination, this study examined the 
impact of communication preference of individuals. The next section discusses the theoretical basis of including communication preference to contribute to this study.

\section{Communication Preferences in the Online Autistic Community}

Literature points out the enormous communication benefits of online interactions in autistic individuals (Davidson, 2008; Rosqvist, Brownlow, \& O’Dell, 2013). In a New York Times article, Harvey Blume (1997a) said, "the impact of the Internet on autistic individuals may one day be compared in magnitude to the spread of sign language among the deaf. By filtering out the sensory overload that impedes communication among autistic individuals, the Internet opens vast new opportunities for exchange." Research shows that not only can others online be sources of information and resources, but also intimate, quality relationships can be developed over the Internet (Wellman \& Gulia, 1999). Blume (1997b) describes the Internet as an important tool in addressing what Wing (1997) would consider one of the 'triads of impairment'- social development. Considering the wide range of language abilities in autistic individuals, the Internet allows individuals to communicate and foster such relationships with greater ease than in face-to-face interactions (Benford \& Standen, 2009), potentially leading to supportive networks and access to friendships and resources. A qualitative study indicates that the Internet reduces nonverbal cues and increases the standardized structure to interpersonal communications, which may contribute to the benefits of such communication for autistic individuals (Burke et al., 2010). 
As is true for all Internet users, autistic adults also benefit from the ability to have relationships with individuals across geographic distance, increasing the likelihood of finding individuals with similar interests (Bagatell, 2007; Jones \& Meldal, 2001; Jordan, 2010), which may contribute to the preference to communicate online rather than face-to-face with individuals with which they do not share communalities. Additionally, many listservs (e.g., Autism-AAD), chat groups (e.g., autistichat), and other interactive websites (e.g., AS-and-Proud-of-It) exist to provide such support for autistic adults and their families, friends, and caregivers. Face-to-face support may not be as effective or accessible as online venues.

To understand how people with autism can maximize their social abilities online, it is necessary to understand Internet communication. Suler (1997) describes the common language and interactions that take place in synchronous (real-time) online communication. Where humor or sarcasm may be confusing in face-to-face interaction for some individuals, use of common emoticons (e.g., , : ), ;), :o, , :(, :D, :p, <3) explicitly convey the intentions of the writer. A common language of acronyms has also developed to supplement emoticons and typical written language in exhibiting emotion and informality, such as LOL (laugh out loud). Additionally, use of different fonts and font styles non-verbally add emphasis or tone to conversation, such as capitalizing whole words or sentences. Since a delayed response is still considered appropriate, a member of an online exchange is free to take time to read, understand, and formulate a response (Benford \& Standen, 2009; Burke et al., 2010; S. Ryan \& Räisänen, 2008; Suler, 1997). Even with the non-verbal cues available through online interactions, 
misinterpretations can often occur (Seymour \& Lupton, 2004). Therefore, Internet users attempt to write with literal meanings and minimal non-verbal cues (Suler, 1997), which parallels the typical communication style of many autistic individuals. Further, patterns in socializing over the Internet create schemas that individuals may use as a guide to their own social behaviors (Shirky, 2003).

Autistic individuals may have a preference for online communication for reasons beyond social and communication differences or the ability to communicate over long distances. Preference for online communication is also discussed in terms of autistic individuals finding a separate safe space from the outside world (Rosqvist et al., 2013; Ryan \& Räisänen, 2008). By utilizing online Autistic communities, individuals felt safer and more welcome than within the constraints of neurotypical face-to-face interactions (Brownlow \& O’Dell, 2006; Rosqvist et al., 2013).

The utility of the Internet for Autistic individuals to communicate is particularly important for contributions to the Autistic self-advocacy movement. Research shows that effective communication is necessary for developing self-advocacy, because it is necessary to express needs and hopes to one other, problem-solve individual and community issues, and become aware of the commonalities, strengths, and needs of the community (Test, Fowler, Wood, Brewer, \& Eddy, 2005). The Internet allows autistic individuals to bypass most communication challenges, allowing for successful communication and opening the opportunity for self-advocacy. Great strides in advocacy and relationship building have occurred online among Autistic individuals, perhaps due to many autistic individuals preferring online communication. 
However, it is also important to recognize the possible difficulties faced by people with disabilities in using the Internet, including visual difficulties and confusing site layout (Burks et al., 2000). Cognitive or neurological differences may create sensitivity to flickering or strobing websites or find navigation through websites more challenging (Brownlow \& O’Dell, 2006). Additionally, individuals with differences in motor skills, inability to physically access computer space without assistance, or other physical difficulties may limit the ability of a person to use the computer or keyboard. By shedding light on the use of the Internet to satisfy communication preference, this study may impact how website creators decided to design websites' such individual may consider the input of an important group of users, autistic individuals with unique preferences and needs for specific website interfaces.

Many of the people with disabilities interviewed for a study by Seymour and Lupton (2004) use the Internet for social interaction and making friends. This finding is in line with studies of broader neurotypical populations who use the Internet mostly for email and social purposes (Nie \& Erbrin, 2000); however, the Internet may be a particularly safe and appropriate venue for social relationships among individuals with disabilities. People with disabilities are sometimes identified as having a disability by others because of physical characteristics, such as using a wheelchair or displaying behaviors associated with having a disability (Seymour \& Lupton, 2004). This forces many people to be "out" about their disability. However, such face-to-face interactions do not always occur on the Internet. The anonymity of the Internet creates the ability to disclose as little or as much information on one's disability as one desires. The 
researchers found that participants emphasized the ability to find and relate to others like themselves, while retaining anonymity and not using physical appearance and presentation as part of identity. Bowker and Tuffin (2002) consider this ability to be an advantage for individuals who may wish to express different parts of their identity in different contexts. Another advantage may be decreased discrimination towards people with disabilities. Guo, Bricout, and Huang (2005) found that $54 \%$ of their respondents with disabilities said there is less discrimination toward people with disabilities online than in face-to-face interactions, suggesting that the option of disclosing disability may decrease discrimination. When specifically considering the Autistic community, using the Internet for communicating with others may serve to lessen differences in social interactions, making language use similar to non-autistic Internet users (Benford \& Standen, 2009; Burke et al., 2010; Newton, Kramer, \& Mclntosh, 2009).

Much literature supports the widely accepted notion that autistic individuals may prefer to communicate in text-based or online venues (Blume, 1997a; Burke et al., 2010; Davidson, 2008; S. Ryan \& Räisänen, 2008). However, no quantitative research has examined communication preferences of autistic individuals and how preference may moderate the relationships between involvement in the online Autistic community and positive outcomes such as Autistic identity and self-determination. The current study sought to understand whether a preference for online communication versus no preference for online communication moderates the relationship between involvement in the online Autistic community and Autistic identity. Additionally, the current study sought to understand whether a preference for online communication versus no 
preference for online communication influences the relationship between involvement in the online Autistic community and self-determination. Understanding the moderating impact of communication preference may provide empirical support for individuals promoting policy or education for autistic individuals. If a preference for online communication increases the impact of involvement in the online Autistic community on Autistic identity or self-determination, it supports increased Internet access and education as well as consideration of individualized interventions based on communication preference.

Additionally, it is unknown whether a preference to communicate online has the same impact on non-autistic adults, creating a need to empirically compare whether communication preference impacts both populations. Empirical support for the greater impact of communication preferences in autistic adults beyond non-autistic adults may illuminate the unique preferences among autistic adults. Such knowledge may inform policy and education designed for autistic individuals who prefer online communication over face-to-face communication. Lastly, the current study examines between-group differences in autistic individuals who prefer online communication versus autistic individuals who do not prefer online community on the relationships among the variables involvement in the online Autistic community, Autistic identity, and selfdetermination. Understanding whether communication preference in autistic individuals impact the strength of relationships among positive variables associated with involvement in the online Autistic community may provide further support for the 
consideration of communication preference in developing education techniques and interventions for autistic individuals.

As the literature has documented, online relationships allow communication among autistic adults to be similar to non-autistic individuals (Benford \& Standen, 2009; Burke et al., 2010; Newton et al., 2009), which led to the formation of a powerful community (Sinclair, 2005), and a strengthened Autistic community and individual identity (Brownlow \& O’Dell, 2006; Kidney, 2012). However, no study has empirically examined a multi-dimensional construct of involvement in the online Autistic community and its relationship to Autistic identity, or the moderation of such a relationship by communication preference. Further, this study examined the relationships amoung involvement in the online Autistic community, Autistic identity, and self-determination in individuals who have a preference for online communication and individuals who do not have a preference for online communication. The next section discusses the construct of Autistic identity.

\section{Autistic Identity}

The Autistic community gained a sense of identity tied to the community's growth and developing ideals and norms, as interplay of new clinical indicators, policies, and systems were informing the traditional and medical views of autism (Ortega, 2009a). The broadening of diagnostic criteria and awareness about autism meant a societal and community-wide reframing of autism, including who identified as autistic. Additionally, organizations formed by self-advocates, parents, and professionals were 
creating differing visions of an Autistic identity (Silverman, 2008). While some organizations framed an Autistic identity as a deficit, others fostered pride and positivity around such an identity. As in other grassroots movements, an Autistic identity within the self-advocacy movement was formed out of a reactionary identity from the deficitbased medical model definition of autism. Further, the developments in identity occurring at the community level had a transactional relationship with the individuals developing a sense of self and identity within the community. Here I discuss the psychological concept of identity and how the transactional theory of Autistic identity was conceptualized in the current study.

Identity is studied as the individual's perception of self and as a dynamic, lifespan process of differentiating oneself from the environment and context (Erikson, 1956; Marcia, 1966). This definition expanded to include the social element of identity, suggesting that people use social categories to identify themselves and others within the same and different categories (Tajfel \& Turner, 1979). Many theories of identity now propose that context must be considered in the development of identity (Adams \& Marshall, 1996). Theories of women's identity (Josselson, 1987), Black identity (Boykin, 1986; Cross, 1971), and LGBT identity (Cass, 1984; D'Augelli, 1994) incorporated elements of social constructionism and context into identity development. Individuals utilizing social constructivist theory understand the world as social artifacts rather than objective realities that interact with historical influences, interactions between individuals, language, politics, and culture. Therefore behavior and identity must be considered within a framework considering all aspects of the context, environment, and 
social order (Archakis \& Tzanne, 2005; Kitzinger, 1989). Online communities can also be described as socially constructed, with no physical boundaries or prescribed physical qualities required for membership (Bagatell, 2010).

Therefore, one must consider the history of the medical perspective of disability and autism as one aspect of the context and social order for a more complete depiction of Autistic identity. Additionally, the online Autistic community is integrally tied to the development of an Autistic identity. The community formed its' group understanding through the Internet beyond the medical discourse surrounding an autism identity before the early 1990s (Ward \& Meyer, 1999). This exchange of personal identity and community identity formation in the online Autistic community is documented in autobiographical accounts and online discourse (Bagatell, 2007; Blume, 1997b; Ortega, 2009a; Silverman, 2008; Yergeau, 2010). As described in previous sections, Autistic individuals seeking the company and relationships of other Autistic individuals serve on a personal level to validate the feelings of differentness autistic individuals felt (Silverman, 2008).

When considering the identity of an individual within a context, one must also acknowledge the dynamic systems of power and oppression that influence and are influenced by developing individuals (Abberley, 1987; Foucault, 1978; Friere, 1972). Barriers exist in society that block out people perceived to be different, such as autistic individuals (Fine \& Asch, 1988). Professionals in the field of autism have defined autism according to a deficit, with something wrong or lacking in autistic individuals. Therefore, the Autistic community must differentiate themselves from non-autistic individuals, 
marginalizing or "othering" themselves from the dominance and power of the majority of the population (Brownlow, 2007). However, with the label of disability or autism, this group may find commonality with one another apart from those without such labels (Fine \& Asch, 1988). Much like the gay and lesbian communities have adopted labels originally constructed as negative (Kitzinger, 1989), the Autistic community has politicized the label of autism, using such a label as a source of pride rather than deficit (Brownlow, 2007), or even describing being autistic as a gift (Ortega, 2009). Identifying as a member of a marginalized and stigmatized community, while having positive repercussions of finding like individuals and belonging, complicates how an individual views societal negative aspects of self with the Autistic community's pride. As all these layers impact the development of identity, one must consider identity as an individual, social, and ecological construct within a transactional model. Thus, Autistic identity, like disability identity, is an interactional, dynamic concept that integrates the biological, cognitive, social, and historical contexts of self (Gill, 1997).

Disability identity is the development of a positive self-concept, understanding and acceptance of the perceptions others have about one's disability or oneself, and understanding of how disability impacts one's life (Olney \& Kim, 2001). Gill's construct of disability identity uniquely uses a social constructionist and transactional model of identity development, which applies to many marginalized identities in society, such as gay and lesbian identity (Cramer \& Gilson, 1999). This model of disability identity can also specifically apply to Autistic identity. Disability identity, therefore, is a developmental, integrative process that occurs on the individual and group level. 
Boykin's (1986) Triple Quandary Theory posits that Black identity forms through socialization into three groups with often competing agendas: mainstream society, Black culture, and minority identity. D'Augelli's (1994) model of LGBT identity development consists of interaction processes of individuals interacting with and shaping their environments as well as responding to them. Similar to other minority identity theories, Gill's theory of disability identity involves individuals forming identity through integration into different social categories while interacting with the broader context of society and the disability community. Gill describes the four types of integration which underlie the process as integration into society ('coming to feel we belong'), integration into the disability community ('coming home'), internal integration of sameness and differentness with society and disability community ('coming together'), and integration of feelings about self and presentation of self ('coming out').

Integrating into society occurs on an individual level as a person asserts his or her right to belong in society (Gill, 1997). On a group or community level, this dimension is reflected in the disability rights movement, Americans with Disabilities Act, and the demand for equal rights and accommodations. Within the Autistic self-advocacy movement, this integration into society is reflected in the actions and events that demanded inclusion and acceptance, such as the Ransom Notes Affair and the spread of Autism Acceptance Day. This process includes the shift from blaming one's disability as creating a separation from "normal" life to redirecting the blame on the makers of noninclusive and unaccommodating environmental conditions. In the online Autistic 
community, this dimension shows in sharing of information on rights and advocacy that can be brought to mainstream society.

The dimension of integrating into the disability community involves overcoming any fears of past memories of disability segregation or stigma associated with mainstream ideas of relationships among people with disabilities (Gill, 1997). Once attitudes about integration with the disability community are overcome, a person may find such integration and relationships rewarding and uniquely strong due to an acceptance and understanding that surpasses many people without the experiences of disabilities. In the online Autistic community, individuals expressed this dimension in solidarity and meaningful relationships members form with one another within communities designed and run by Autistic adults. Autistic individuals reflect in their mixed feelings of receiving an autism diagnosis or come to the realization that they are autistic. It involves acceptance of being a member of a stigmatized, minority population. The dimension of integration into the Autistic community involves what autistic individuals describe as finally feeling as though they have a place or a group in which they share a common language and common life experiences.

Internal integration of sameness and differentness with society and disability community involves resolving the conflict of self as broken or not whole with a sense of pride and belonging (Gill, 1997). This also relates to supporting self-esteem and the ability to perceive the self as a part of both the disability community and mainstream society. Since a true sense of belonging to these communities relies on the resolution of 
the fractured parts of the self, the stability and progress of integrating into society and integrating into the disability community may be dependent on this dimension.

Lastly, integration of feelings about self and presentation of self involves resolving the private perception of self with the ideal image presented to others (Gill, 1997). The integration is the final stage of identity that results in stability in how comfortable individuals are with themselves. This dimension is a lack of internal conflict or discomfort with how individuals perceive themselves as well as within social environments. Ortega (Ortega, 2009a) discusses "being autistic or having autism" in the sense that individuals come to see autism as being an essential part of their being, rather than an add-on or one aspect of their identity.

Qualitative research supports Gill's theory of disability identity among individuals with spinal cord injuries (Hernandez, 2005) and among queer women with disabilities (Whitney, 2006). Gill (1997) developed a quantitative scale measuring disability identity according to her theory of disability identity that has been applied to individuals with spinal cord injuries (Lee, Lee, Rhee, Shin, \& Lim, 2008). Disability identity was positively correlated with higher social integration, higher social support, and lower depression in the sample. However, no research examined the psychometrics properties of the disability identity scale. This theory has only been applied quantitatively within the specific population of autistic adults in my thesis project (Kidney, 2012), with a subset of the sample utilized for the current study. In the subset of the current sample, the adapted Autistic Identity Scale showed acceptable internal consistency reliability with an alpha of .73; however, no further psychometric qualities of the scale were examined. 
The current study explores the factor structure of the scale to determine whether the scale retains the theoretical dimensionality of Autistic identity and whether the scale exhibits good psychometric properties in the current sample.

My thesis project provided evidence for a positive relationship between Autistic identity and sense of community in the online Autistic community (Kidney, 2012). Further, a positive relationship between the importance of involvement in the online Autistic community and Autistic identity was supported, as well as a positive relationship between the importance of involvement in the online Autistic community and sense of community. Other research has also shown that identity development is related to Internet use (Turkle, 1995) and sense of community (Obst \& White, 2005). Further, while Autistic identity has been studied qualitatively (Brownlow, 2007a; MacLeod, Lewis, \& Robertson, 2013) and with a subset of the study sample (Kidney, 2012), no quantitative studies has explored how multiple indicators of involvement in the online Autistic community is related to Autistic identity, or how Autistic identity is related to self-determination.

\section{Self-Determination}

Woven into the self-advocacy movement of the Autistic community and other disability communities is the necessity for self-determination to guide rights and values (Field \& Hoffman, 1999; Wehmeyer, Bersani, \& Gagne, 2000). The current study examines self-determination in autistic adults in order to shed light on how involvement in the online Autistic community may lead to positive outcomes in the lives of autistic 
adults. While definitions of self-determination vary widely (Malian \& Nevin, 2002), selfdetermination is personal agency or self-directed action in making one's own decisions and working towards personal fulfillment (Powers et al., 2012; Shogren et al., 2008; Wehmeyer, 2005; Wehmeyer, Shogren, Zager, Smith, \& Simpson, 2010). Specifically, self-determination refers to understanding of one's strengths, limitations, needs and preferences (Cobb, Lehmann, Newman-gonchar, \& Alwell, 2009), as well as the power to make choices and self-directed actions as well as the right to take risks (Powers et al., 2012). Field, Martin, Miller, Ward, and Wehmeyer (1998) define self-determination as: "a combination of skills, knowledge, and beliefs that enable a person to engage in goaldirected, self-regulated, autonomous behavior. An understanding of one's strengths and limitation, together with a belief of oneself as capable and effective are essential to selfdetermination. When acting on the basis of these skills and attitudes, individuals have a greater ability to take control of their lives and assume the role of successful adults in our society." (p. 2). Self-determination includes an interaction between internal and external characteristics of empowerment, consciousness about oneself, behaviors and actions towards obtaining needs and goals, and the ability to find support to meet such needs and goals (Eisenman \& Chamberlin, 2001; Whitney-Thomas \& Moloney, 2001). Self-determination requires an awareness of what needs and goals are present within oneself, the power to pursue such needs and goals, and the ability and/or supports to achieve them.

Literature shows that self- determination has a powerful positive impact on youth with disabilities (Wehmeyer \& Palmer 2003). Self-determination is also related to 
higher quality of life and life satisfaction (Cagle, 2006; Lachapelle et al., 2005; Nota, Ferrari, Soresi, \& Wehmeyer, 2007) and employment outcomes (Lachapelle et al., 2005; Nota et al., 2007; Shogren et al., 2008; Wehmeyer \& Schwartz, 1998) in adults with disabilities. Self-determination is related to employment, health care access, financial independence and independent living in individuals with disabilities after graduation (Wehmeyer \& Palmer, 2003). Further, a synthesis of narratives and meta-analyses on the topic of students with disabilities provides evidence to support various benefits of self-determination promotion (Cobb et al., 2009). Other systematic reviews examine the impact of interventions for self-determination in individuals with disabilities (Algozzine, Browder, Karvonen, Test, \& Wood, 2001; Chambers, Wehmeyer, Saito, Lida, \& Lee, n.d.; Malian \& Nevin, 2002) and interventions focusing on students with severe disabilities (Wood, Fowler, Uphold, \& Test, 2005). Additional systematic reviews focus on the efficacy of self-determination interventions on the academic outcomes of students with disabilities (Konrad, Fowler, Walker, Test, \& Wood; Fowler, Konrad, Walker, Test, \& Wood, 2007; Bae, 2007). Beyond studying the outcomes of self-determination, literature supports the role of culture and context as important components to fostering self-determination in Dine (Navajo) individuals (Franklin, Turnbull, Wehmeyer, \& Blackmountain, 2004), youth with disabilities in foster care (Greenen, Powers, Hogansen, \& Pittman, 2007), and in all individuals with disabilities (Wehmeyer et al., 2011).

In addition to communities viewing self-determination as essential and research supporting such sentiments, legislation passed indicates the need to integrate self- 
determination into policy impacting individuals with disabilities. This includes the Rehabilitation Acts of 1992 (Baker, Horner, Sappington \& Ard, 2000) and 1998, the Individuals with Disabilities Education Acts of 1990 and 1997 (Wood, Karovonen, Test, Browder, \& Algozzine, 2004), the IDEA reauthorization in 1990 which mandated student's perspectives be taken into account for transition programs (Bremer, Kachgal, \& Schoeller, 2003), and research and development initiatives supporting 26 projects from 1991 to 1993 (Ward \& Meyer, 1999). Empirical evidence shows that selfdetermination is a valuable skill that is teachable to individuals with disabilities in schools and at home (Malian \& Nevin 2002). Therefore, an integration of understanding of the phenomenon of self-determination into legislation is promising, given the widely known positive outcomes of obtaining such skills.

Extant literature on self-determination in autistic individuals focuses on children and students in special education programs and family supports for developing selfdetermination in autistic adolescents (Field \& Hoffman, 1999). Such literature shows that autistic individuals exhibit less self-determination than peers without disabilities (Wehmeyer \& Shogren, 2008). However, such research lacks contextual lifespan approaches and autistic perspectives, and contains assumptions that autistic individuals may not have the ability to meaningfully comprehend the application and worth of selfdetermination and its associated skills (Fullerton \& Coyne, 1999; Wehmeyer et al., 2010).

The concept and construct of self-determination varies slightly depending on the field of study, population of interest, and application of the theory (Shogren et al., 
2008). Despite the popularity in examining outcomes and interventions related to selfdetermination, the varying theories of self-determination create common theoretical misunderstandings and a lack of clarity regarding theory and measurement of the construct (Shogren et al., 2008), resulting in inconsistencies across theory and measurement. Different perspectives view self-determination as a developmental phenomenon, an ecological phenomenon, a skill or competency, a status, and a psychological phenomenon (Malian \& Nevin 2002; Test, Fowler, Wood, Brewer, \& Eddy, 2005). Self-determination changes throughout the lifespan, with changes occurring in multiple realms of the individual's life, including emotional, social, communicatory, and behavioral (Malian \& Nevin 2002). Here I discuss a number of self-determination theories, including self-determination from a functional perspective (Field, 1996) and theories most often applied in disability literature (Wehmeyer, 1996; 1997; 1999; 2001). Then, I discuss Ryan and Deci's (2000) theory of self-determination within a developmental framework and approach to conceptualizing motivation in psychology, which applies to the current study.

Some theories of self-determination take on a functional perspective, with less emphasis on psychological and internal innate qualities of an individual predicting selfdetermined behavior, and more emphasis on individuals' actions, feelings, beliefs, skills, and functioning within environments. Field (1996) characterizes a theory of selfdetermination by four models or different perspectives of the sources selfdetermination. The different models include an individually-focused perspective based on an individual's beliefs, knowledge and skills; a perspective based on the outcomes of 
self-determination in adults; a self-regulation perspective; and a perspective based on ecological theory that posits that environmental factors create self-determination in individuals. Abery and Stancliffe (1996) also utilize such an ecological theory in their ecological model of self-determination in which individuals must determine the effective behaviors, skills, and attitudes that are appropriate in order to achieve control over important aspects of their own lives. Abery and Stancliffe derived this perspective from Bronfenbrenner's ecological perspective $(1979,1989)$. Additionally, Mithaug, Mithuag, and colleagues developed a theoretical framework (2003) in which selfdetermination is an interaction between individuals knowing and understanding their own capacity to create change within any given opportunity. Such a match between a person's assessment of capacities and the opportunity to effectively implement them is a self-regulatory, transactional theory that requires consideration of the personenvironment fit.

Wehmeyer $(1996 ; 1997 ; 1999 ; 2001)$ proposed a different theory of selfdetermination that is frequently applied in the disability literature. Wehmeyer's theory of self-determination compromises dispositional characteristics that allow individuals to consciously perform actions and make decisions by themselves in order to contribute to one's quality of life. Wehmeyer identifies the four domains, or essential characteristics, of self-determined behavior as autonomy, self-regulation, psychological empowerment, and self-realization. Autonomous functioning is an individual acting towards one's own preferences without unwarranted external influence. Self-regulation is individuals selfmanaging aspects of their own lives, including goals and problem-solving. Psychological 
empowerment is an individual controlling important aspects of her own life and possessing, including having the necessary skills to have such control then implementing such skills. Finally, self-realization is knowledge of one's strengths and limitations. These four characteristics of behavior emphasize the consciousness of the behavior, and the ability to act upon the behavior. This theory is functional, and takes a lifespan approach to developing self-determination via the acquisition of interrelated skills and attitudes that compromise the elements of self-determined behavior. These elements are choicemaking skills, decision-making skills, self-monitoring skills, self-advocacy skills, an internal locus of control, perceptions of self-efficacy, and outcome expectancy, selfawareness, and self-knowledge (Wehmeyer, 1996).

Lastly, Ryan and Deci's (2000) self-determination theory is an approach to motivation within personality psychology that considers individuals' internal resources for development and self-regulation. The theory is comprised of developmental components interacting with innate psychological needs of autonomy, competence, and relatedness within a context that supports or blocks the processes. Self-determination theory contains the underlying assumption that all humans strive for growth and integration of self (Ryan \& Deci, 2000). Within this theory, the psychological needs that must develop within an individual include competence, relatedness, and autonomy. If the environment an individual develops within is supportive, the presence of these three psychological needs will result in a self-determined individual. Ryan and Deci's theory is empirically tied to theories of intrinsic and extrinsic motivation, as well as a spectrum of self-regulation. As motivation and self-regulation become more intrinsic to 
individuals, the more self-determined they behave. Such behavior is also transaction ally determined, with the absence of competence, relatedness, and autonomy resulting in less motivation or controlled motivation creating negative pressure to behave in a particular manner (Ryan \& Deci, 2012).

Ryan and Deci's (2000) self-determination theory posits that an individual must develop critical psychological components necessary for self-determination, which interact with the environment to foster self-determination. The online Autistic community is described as an environment for autistic individuals to support communication and social differences and preferences, resulting in the development of positive outcomes such as sense of community and Autistic identity (Kidney, 2012). Since Ryan and Deci's (2000) self-determination theory focuses on the importance of individual characteristics interacting with the environment, an integral aspect of the study, this theory is most relevant as a theoretical framework and basis for measurement of the study. Further, in relation to the concepts utilized for the current study, Ryan and Deci's theory is closely aligned with concepts of identity and sense of community, which are psychological concepts that also consider interaction with the context. Specifically, identity development occurs concurrently with other development. Therefore, identity is an integral component of the self that interacts with basic psychological needs critical for self-determination (Bart \& Maarten, 2011). Ryan and Deci's self-determination theory revolves around the three basic psychological needs of autonomy, competence, and relatedness (Ryan \& Deci, 2000). 
The basic psychological need of autonomy is a sense of volition, willingness, and congruence, wherein individuals decide on the behavior they engage in (Ryan \& Deci, 2000). A person high in autonomy has high internal locus of causality and competence (perception of the source of actions and behaviors), which is separate but related to locus of control, whether individuals have control over their own outcomes (Deci \& Ryan, 1985). That is, autonomous individuals believe themselves to be in charge of their own actions, and individuals with high locus of control believe themselves to be in control of what happens to them in life. Autonomy is more aligned with perceptions of activities and behaviors rather than outcomes, which are aligned with locus of control. Ryff adds that autonomy also refers to the ability to evaluate the self by standards within the individual rather than looking for outside social pressures to conform (Ryff, 1989). For individuals who require accommodations or are interdependent with others for support in certain contexts, defining ones' own control over their own activities and behaviors is central to defining autonomy rather than how others' perceptions of a person's abilities.

Autonomy is important when considering identity development, which is relevant to examining Autistic identity in the current study. A study supports autonomy as impacting identity exploration and well-being, with individuals exhibiting high autonomy having higher identity commitment, identity integration, and self-esteem (Luyckx et al., 2007). Evidence supporting a positive relationship between Autistic identity and self-determination, including autonomy, suggests that similarly positive outcomes may be seen in Autistic individuals involved in the online Autistic community. 
Within a lifespan and historical perspective, autonomy should increase with development, yet is impacted by societal oppression (Ryan \& Deci, 2012). Such a balance of developmental abilities and societal oppression is relevant and evident in the Autistic community, where many autistic individuals lack a sense of autonomy due to marginalization, stigma, and oppression. However, individuals in the online Autistic community may encounter an environment in which they may have more control and comfort with interactions, information-seeking, relationships, and decisions, resulting in increased autonomy in the individual. As an individual becomes more involved in the online Autistic community, autonomy may intrinsically increase in the individual, and impact over aspects of life both online and offline. Further, a person high in autonomy actively seeks out opportunities in line with their personal values and interests (Luyckx et al., 2007). Therefore, individuals more involved in the online Autistic community likely exhibit higher levels of autonomy, based on their autonomous behavior of seeking a community of individuals sharing common experiences and qualities.

Ryan and Deci's self-determination theory also contains the psychological need of competence (Ryan \& Deci, 2000). Competence involves individuals' abilities and effectiveness in controlling aspects of their environments and lives (Deci \& Vansteenkiste, 2004). Ryff defines the parallel concept of environmental mastery as knowing how to choose, create, or control one's activities and contexts in order to utilize one's own strengths (Ryff, 1989). Within the framework of the socio-ecological model, the psychological need of competence exists purely as an interaction between the individual and context. In the context of the online Autistic community, autistic 
individuals navigate and control the amount of information presented on them, exhibiting individual competence, while also banding together as a self-advocacy movement to promote the rights and needs of the Autistic community. These actions indicate the competence of participating autistic individuals. As with the psychological need of autonomy, competence may increase as a result of a transactional relationship with the environment, while seeping into other aspects of life. Therefore, involvement in the online Autistic community is likely positively related to competence.

A related construct to the psychological need of competence is locus of control, which is the amount of control individuals believe they have in their lives (Rotter, 1968). Individuals who have high internal locus of control have high competence. Studies show a positive relationship between internal locus of control and racial identity development among African American adolescents (Cosby, 1999) and internal locus of control and ethnic identity development among college students from ethnic minority groups (Lee, 2012). The development of racial or ethnic identity is similar to disability and Autistic identity development (Boykin, 1986; Cross, 1971). Therefore, the psychological need of competence is likely positively related to Autistic identity in the current study. In Lee's study (2012) of locus of control and ethnic minority identity, locus of control is positively related to identity commitment and slightly negatively related to identity exploration. Identity exploration is a phase in which an individual experiences a crisis of identity and seeks out an identity (Marcia, 1980). Identity exploration is conceptually related to identity commitment, which is when the individual is settled and comfortable within an identity (Marcia). In terms of the current study, autistic individuals may have higher 
internal locus of control or perceived competence if they have committed to their identity rather than still exploring their Autistic identity. Higher involvement in the online Autistic community may be an indication of identity commitment, specifically years spent involved in the community, importance of involvement, and sense of community.

Lastly, Ryan and Deci's self-determination theory contains the psychological need of relatedness (Ryan \& Deci, 2000). Relatedness concerns the individual's need to interact with and meaningfully connect to other people (Deci \& Vansteenkiste, 2004). Autistic individuals express this psychological need in the beginning of the self-advocacy movement as a need to connect with like others (Sinclair, 2005; D. Williams, 1994). Further, in a subset of the sample utilized for the current study, involvement in the online Autistic community is positively related to sense of community and all of the subscales of the $\mathrm{SCl}-2$, including the dimension of shared emotional connection (Kidney, 2012), a concept tied closely to relatedness. Shared emotional connection is the sense of shared history and understanding among members (McMillan \& Chavis, 1986). McMillan and Chavis described this connection as requiring close relationships and identification with members to feel a connection. This construct is particularly relevant in the Autistic community, which, as described in Chapter II, has a shared history of the development of the Autistic self-advocacy movement, events in the Autistic community that brought them closer together as a community, and common understandings of words, symbols, and events related to their community. Such a shared emotional connection is theoretically tied to the relatedness within the current study. 
Researchers have applied and measured self-determination theory by various metrics. Many correlational studies, intervention studies, and theoretical articles on the topic of individuals with disabilities apply Wehmeyer's theory. Further, it is the only theory that research applied directly to autistic individuals, though these articles focused on autistic grade school and high school students and adolescents (Wehmeyer et al., 2010; Wehmeyer \& Smith, 2011). Along with the various theories of selfdetermination throughout the literature, the literature recommends and utilizes various modes and instruments to measure self-determination in individuals with disabilities (Field \& Hoffman, 2007; Hoffman, Field, \& Sawilowsky, 1996; Martin \& Marshall, 1996; Shogren et al., 2008; Wehmeyer \& Kelchner, 1995; Wolman, Campeau, Dubous, Mithaug, \& Marshall, 1996), particularly the Arc's Self-Determination Scale (Wehmeyer \& Kelchner, 1995) and the American Institutes for Research Self-Determination Scale (Wolman et al., 1994). Many studies utilize Ryan and Deci's (2000) self-determination theory in empirical studies. Empirical evidence supports the relationships between the psychological needs necessary for self-determination development (autonomy, competence, and relatedness) and the positive outcomes of well-being, health, healthy behaviors (Baard, Deci, \& Ryan, 2004; Kasser \& Ryan, 1999; Williams et al., 2006; Williams, Freedman, \& Deci, 1998).

Despite consistent use of Ryan and Deci's theory of self-determination, measures within such studies have varied, including use of the Self-Determination Scale (Sheldon, Ryan, \& Reis, 1996), and specifically measuring the basic psychological needs of autonomy, competence, and relatedness within specific contexts, such as in the Basic 
Need Satisfaction at Work Scale (Deci et al. 2001; Johnston \& Finney, 2010), Basic Need

Satisfaction in Relationships Scale (LaGuardia, Ryan, Couchman, \& Deci, 2000), and Basic Psychological Needs in Exercise Scale (Vlachopoulos \& Michailidou, 2006). Other measures that apply Deci and Ryan's theory focus on specific dimensions of the basic needs, such as autonomy with the General Causality Orientations Scale (Deci \& Ryan, 1985), which presents vignettes of life situations and asks for the extent to which a response is autonomously oriented. Studies often use this type of measure tandem with autonomy support measures (Deci \& Ryan 1989; Gagne, 2003), which indicates the amount of support given by others, such as managers or parents, to support autonomous decision-making in individuals (Baard et al., 2004).

Measures that adapt all three of the basic psychological needs within a specific context are most relevant to the current study, which utilizes autonomy, competence, and relatedness measures to indicate the extent of psychological needs that may contribute to self-determination due to the supportive environment of the online Autistic community. The current study measured one of the dimensions, relatedness, with the $\mathrm{SCl}-2$ subscale of shared emotional connection (Chavis et al., 2008), which identifies the context of the online Autistic community as the community to think about when responding to the items. Therefore, the dimension of relatedness in the current study measures considers the context, as in other measures of the basic psychological needs for self-determination (Deci et al., 2001; LaGuardia, Ryan, Couchman, \& Deci, 2000; Vlachopoulous \& Michailidou, 2006). The literature on the Basic Need Satisfaction Scale (BNSS) shows that the scale was psychometrically examined and determined to 
have high positive correlations with the Psychological Well-Being Scales dimensions of autonomy and environmental mastery, with particularly strong relationships with the autonomy and competence dimensions of the BNSS, respectively (Johnston \& Finney, 2010). The current study is the first to apply the theory of self-determination in autistic adults in online communities, the first to apply Ryan and Deci's (2000) selfdetermination theory in the population, and the first to utilize a composite measure of the three domains from adapted measures of autonomy, competence, and relatedness. Table 1 displays the constructs and measures of self-determination utilized in the study. In summary, self-determination is an integral aspect of involvement in selfadvocacy for individuals with disabilities and Autistic individuals (Wehmeyer et al., 2000). Autistic advocates promote a strengths-based approach to research that would illuminate positive aspects of the community and promote inclusion (Robertson, 2010). Self-determination has a powerful positive impact on individuals with disabilities, including a higher quality of life and life satisfaction (Cagle, 2006; Lachapelle et al., 2005; Nota, Ferrari, Soresi, \& Wehmeyer, 2007) and employment outcomes (Lachapelle et al., 2005; Shogren et al., 2008, Wehmeyer \& Schwartz, 1998). Legislation also passed indicating the need to integrate self-determination into policy impacting individuals with disabilities (Baker, Horner, Sappington, \& Ard, 2000; Wood, Karovonen, Test, Browser, \& Algozzine, 2004) and research and development initiatives (Ward \& Meyer, 1999). However, no previous research focuses on self-determination in the online Autistic community. 
The current study examined the relationship between involvement in the online Autistic community and self-determination, as well as examines the influence of communication preference on the relationship. Then, the study examined the influence of population type (autistic or non-autistic) and communication preference in the relationship between involvement in the online community and self-determination. Lastly, the study examined the relationships among involvement in the online Autistic community, Autistic identity, and self-determination in a comparison model between autistic individuals who prefer to communicate online and autistic individuals who do not have a preference for online communication to determine good model fit and differences between the groups with and without preference for online communication. The study offers the first empirical information on self-determination in the online Autistic community. The inclusion of self-determination in the study may influence policies, education, techniques, and interventions that aim to promote selfdetermination in autistic individuals by providing support for involvement in the online Autistic community with consideration of communication preference. By utilizing a comparison between autistic individuals and non-autistic individuals for one analysis, the study may also promote the unique needs and preferences of autistic individuals beyond non-autistic individuals who prefer to communicate online.

\section{Involvement in the Online Autistic Community, Identity, Community, and Well-Being}

The literature provides qualitative evidence for the benefits of involvement in the online Autistic community, anecdotal information on how involvement in the 
community may have impacted self-determination, and anecdotal information on the impact of unique communication preferences. However, the current study's aims most closely align with a study involving a subset of the current study data in my master's thesis study, entitled Involvement in the Online Autistic Community, Identity, Community, and Well-Being. The study focused on the online Autistic community and how the importance and value of involvement in it is related to Autistic identity, sense of community, and psychological well-being. AASPIRE conducted the Internet Use, Community, and Well-Being Study and collected data from 72 autistic adults online. I hypothesized that the importance and value of involvement in the online Autistic community would be positively related to Autistic Identity and sense of community, Autistic Identity and sense of community would be positively related and Autistic identity and sense of community would be positively related to psychological well-being. It was also hypothesized that the positive relationship between the importance and value of involvement in the online Autistic community and psychological well-being would be mediated by Autistic identity and sense of community. Israel and colleagues' (1998) six CBPR principle involves a cyclical and iterative process in research in which research develops over time and continues to reflect upon problem definition, the relevance of research questions, and building upon findings. Therefore, it is imperative that I work closely with the research team to examine thesis findings, revisit the utility of constructs, measures, and relationships among them, and extend findings with a larger sample and improved measures and analyses. Here I discuss my thesis findings and how the current study built upon them. 
The thesis study examined correlations among the hypothesized relationships, and used a mediated regression model (Baron \& Kenny, 1986) to explore the relationship between the importance and value of involvement in the online Autistic community and psychological well-being with Autistic identity and sense of community as mediators. The study supported significant relationships between the importance and value of involvement in the online Autistic community and Autistic identity $(r=.37$, $p<.01)$, between the importance and value of involvement in the online Autistic community and sense of community $(r=.45, p<.01)$, and between Autistic identity and sense of community $(r=.42, p<.01)$. The study found no significant findings between Autistic identity and well-being or between sense of community and well-being. As a first step to test the mediated regression models, I regressed psychological well-being on the importance and value of involvement in the online Autistic community. The regression was not significant $[\beta=-0.03 ; t(55)=-0.16 ; p=.872 ; 95 \% \mathrm{Cl}=-0.182,0.142$; $f^{2}=.07 ;$ power=.40]; therefore the hypothesized model was not significant.

Previous research indicates Internet use, involvement in social contexts, and involvement in social movements are related to identity development (Gamson, 1992; Hendry, 1983; Turkle, 1995). Further, the Internet is a hub for creating personal relationships, giving and receiving social support, and developing sense of community (Brownlow \& O’Dell, 2002; Obst, Zinkiewicz, \& Smith, 2002a; Turkle, 1995; Wellman \& Gulia, 1999). My thesis study supported these relationships between involvement and identity and involvement and sense of community, and contributed to the literature by examining the relationships within the context of the online Autistic community. 
However, the measurement of involvement in the online Autistic community was limited to a one-item variable that measures the value and importance of involvement in the online Autistic community. As previously discussed, other indicators of involvement, including frequency of involvement, years of involvement, and sense of community subscales may lead to a more accurate measure of involvement in the online community. This dissertation study builds on findings from my thesis study that indicate that involvement in the online community contributes to positive outcomes by utilizing a data-driven multidimensional construct of involvement. The correlation between involvement in the online community and Autistic identity was examined again in the current study to identify whether the relationship exists with the improved measure of involvement.

The non-significant findings of my thesis study also provide rationale for further analyses and understanding of the full study sample. Literature supports a positive relationships among involvement in online communities, identity, sense of community, and well-being (Barak \& Sadovsky, 2008; Boase, Horrigan, Wellman, \& Rainie, 2006; Rohall, Cotton, \& Morgan, 2002). However, I found no significant relationships between Autistic identity and well-being, sense of community and well-being, or involvement in the online Autistic community and well-being in the mediated regression model. The size of the thesis study sample made sophisticated analyses that modeled the complex interrelationships among the variables impossible, which may have contributed to nonsignificant findings. Further, analyses including the well-being measure indicated lower levels of power, which also was impacted by the small sample size. Community partners 
also noted that turmoil occurring within the community at the time of data collection may have impacted responses to items asking about their well-being and the online Autistic community, which may have lead to non-significant findings. Lastly, the study utilized only three out of six of Ryff's (2001) Psychological Well-Being Scales. Perhaps the exclusion of three of the scales contributed to an incomplete measure of well-being that should be re-evaluated in future research. In the current study, I explored selfdetermination using two of the scales, autonomy and environmental mastery, in addition to the sense of community subscale of shared emotional connection, in order to construct a more complete outcome measurement to illuminate positive outcomes of involvement in the online Autistic community.

In addition to limitations in sample size, power, statistical modeling, and measurement, Involvement in the Online Autistic Community, Identity, Community, and Well-Being lacked any comparison group. Involvement in the online Autistic community may provide autistic individuals opportunities to develop Autistic identity and selfdetermination, but it is unknown whether positive outcomes are unique to autistic individuals. Autistic individuals have a unique shared history online, and many share challenges in socializing and communication that may be reduced online. Empirically supporting that involvement in the online community has greater impact on autistic individuals than non-autistic individuals illuminates the benefits of the online community beyond the benefits experienced by neurotypical Internet users.

My thesis study was the first study to empirically examine relationships among involvement in the online Autistic community, Autistic identity, sense of community, 
and well-being. Findings from the study provide an important first step to further understand the topic of involvement in the online Autistic community. This dissertation study took the next step by addressing many of the limitations of the study to expand on empirical findings about the positive outcomes of involvement in the online Autistic community and whether communication preferences influence such relationships.

\section{Summary}

This chapter contains a review of the literature relevant to the current study, focusing on topics of involvement in the online Autistic community, communication preferences, Autistic identity, and self-determination. Here I summarize the major points of the literature review before describing the research questions and hypotheses.

The increase of the availability, use, and accessibility of the Internet lead to the development of an online Autistic community which provides social connections (McKenna \& Bargh, 1998), information about and for autistic individuals (Jones, Zahl, \& Huws, 2001), and opportunities for self-advocacy (Kras, 2009). Involvement in the online community is a multi-dimensional construct that contains four domains: frequency of involvement, years of involvement, sense of community, and importance of involvement. The dimensions of involvement in the online community are separately associated with social connections (Boase et al., 2006; Kraut et al., 2002), increased activities performed online (Gallup, 2009; Nie \& Erbrin, 2000), community involvement (Allen \& Allen, 1987), and Autistic identity (Kidney, 2012). The current study is the first study to create and utilize a data-driven multi-dimensional construct of involvement in 
the online community. Examining whether the multi-dimensional construct is related to Autistic identity and self-determination sheds light on the online Autistic community as well as the developed measure.

In addition to involvement in the online community, this study examined communication preference. The Internet allows those who prefer to communicate online to foster important relationships and communities with others across any geographic distance (Bagatell, 2007; Jordan, 2010). Due to the communication benefits, many autistic individuals have anecdotally expressed a preference for online or textbased communication over face-to-face interactions (Blume, 1997b; Burke et al., 2010; Davidson, 2008). Despite empirical evidence supporting negative outcomes for individuals who prefer online communication, the current study examined the online community through a perspective that unique communication styles are not innately negative, but may promote positive outcomes within supportive contexts online. The study examined how preferring online communication versus not preferring online community influences the relationships between involvement in the online Autistic community and involvement in the community and self-determination. Examining communication preference in the current study provided evidence of whether communication preference influences the relationships among involvement in the online community, Autistic identity, and self-determination.

The Autistic community gained a sense of identity tied to the community's growth and developing ideals and norms, as interplay of new clinical indicators, policies, and systems informed the traditional and medical views of autism (Ortega, 2009a). The 
exchange of personal identity and community identity formation in the online Autistic community is documented in autobiographical accounts and online discourse (Bagatell, 2007; Blume, 1997a; Ortega, 2009a; Silverman, 2008; Yergeau, 2010). Gill's construct of disability identity uniquely uses a social constructionist and transactional model of identity development (Cramer \& Gilson, 1999). This model of disability identity also applies to Autistic Identity. The positive relationship between involvement in the online Autistic community and Autistic identity is supported in a subset of the current study sample (Kidney, 2012). While qualitative research examined identity in the online Autistic community (Bagatell, 2007), no other studies quantitatively examine Autistic identity in the online Autistic community, particularly as related to the additional concepts of communication preference and self-determination. Therefore, the current study examined these relationships. Since qualitative and quantitative research support positive relationships between involvement in the online Autistic community and Autistic identity, the current study aligns with the existing literature and hypothesizes that the relationship between the constructs are positive.

The current study also examined self-determination. The self-advocacy movement of the Autistic community and other disability communities see selfdetermination as necessary to guide individual rights and values (Field \& Hoffman, 1999; Wehmeyer, Bersani, \& Gagne, 2000). Despite the widespread empirical support for selfdetermination in individuals with disabilities, specifically in education and transitional programs, little empirical work focuses specifically on self-determination in the Autistic community. Ryan and Deci's (2000) self-determination theory is an approach to 
motivation and personality psychology that considers individuals' internal resources for development and self-regulation. The theory is comprised of developmental components interacting with innate psychological needs of autonomy, competence, and relatedness within a context that supports or blocks the processes. Since the online Autistic community is a context that supports communication and the fostering of supportive community (Brownlow \& O'Dell, 2006; Kidney, 2012), the potential exists to develop and promote self-determination in the autistic members of the community. Understanding the impact of involvement in the online Autistic community and the consideration of communication preferences on self-determination may illuminate information on the importance of online contexts in fostering self-determination. Further, understanding how such a relationship is unique or stronger than in nonautistic adults may provide further evidence of the benefits of involvement in the online community for autistic individuals. Therefore, the current study examined these relationships. Since the literature has shown positive relationships between belonging to supportive communities and self-determination, this study hypothesized that the relationship between involvement in the online Autistic community and selfdetermination is positive.

The current study responded to the need for research that reduces stigma in the Autistic community, promotes positive outcomes, and may impact access to Internet, education, and effective interventions. In a subset of the current sample, findings support positive relationships between involvement in the online Autistic community and Autistic identity, between involvement in the online Autistic community and sense 
of community, and Autistic identity and sense of community (Kidney, 2012). One of the objectives of the current study was accomplished by further examining the relationship between involvement in the online Autistic community and Autistic identity in a larger sample, with improvement of the involvement in the online Autistic community variable through use of a multi-dimensional composite variable. The current study also examined whether communication preference may influence the relationship between the constructs. Further, the current study examined the relationship between involvement in the online Autistic community and self-determination. The study examined the impact of communication preference on the relationship between involvement in the online community and self-determination and will compare between the populations of autistic adults and non-autistic adults to further understand the unique preferences and needs of the Autistic community beyond neurotypical online community-seekers.

Finally, the current study examined the relationships among involvement in the online Autistic community, Autistic identity, and self-determination in autistic individuals who prefer online communication versus autistic individuals who do not prefer online communication in order to understand the impact of communication preference on the relationships among variables. Next I present the research questions and hypotheses of the study.

\section{Research Questions and Hypotheses}

This study had one overarching aim and four specific research questions. The study aimed to examine the relationships among involvement in online communities 
and Autistic identity and self-determination, as well as examine the impact of communication preference on the relationships. Specifically, the study examined the relationships between involvement in the online Autistic community and Autistic identity and whether communication preference influences the relationship. The study also examined the relationship between Autistic identity and self-determination and whether communication preference influences the relationship, as well as whether communication preference influences the relationship in autistic individuals compared to non-autistic individuals. Lastly, the study examined the impact of communication preference on the relationships among involvement in the online Autistic community, Autistic identity, and self-determination in autistic individuals. Here, I describe the research questions and hypotheses of the study.

\section{Research Question One}

The first research question addressed the relationship between involvement in the online Autistic community and Autistic Identity, and whether the relationship is moderated by communication preference. The study operationalized communication preference as a dichotomous variable asking whether a person has a preference for online communication or does not have a preference for online communication. I operationalized Involvement in the online Autistic community in this study as a multidimensional variable containing four weighted domains: the frequency of involvement in the online Autistic community, the number of years involved in the online Autistic community, sense of community with the online Autistic community, and importance of involvement in the online Autistic community. Specifically, the first 
research question asked: How is involvement in the online Autistic community related to Autistic identity, and how does communication preference moderate the relationship? I predicted that involvement in the online Autistic community will be positively related to Autistic identity, and communication preference will moderate the relationship. Specifically, involvement in the online Autistic community will be positively related to Autistic identity $(H 1.1)$ and that communication preference will moderate the relationship between involvement in the online Autistic community and Autistic identity (H 1.2). See Figure 2 for a representation of Hypothesis 1.

Hypothesis 1.1. Involvement in the online Autistic community will be positively related to Autistic identity.

Hypothesis 1.2. Communication preference will moderate the relationship between involvement in the online Autistic community and Autistic identity, with a more positive relationship between involvement in the online Autistic community and Autistic identity when a person prefers online communication over when a person does not prefer online communication.

Research Question Two

The second research question addressed the relationship between involvement in the online Autistic community and self-determination, and how the relationship is moderated by communication preference. The study operationalized self-determination as autonomy, competence, and relatedness. Specifically, the second research question asked: How is involvement in the online Autistic community related to self- 
determination, and how does communication preference moderate the relationship? I predicted that there will be a positive relationship between involvement in the online Autistic community and self-determination $(\mathrm{H} 2.1)$ and that communication preference will moderate the relationship between involvement in the online Autistic community and self-determination (H 2.2). See Figure 3 for a representation of Hypothesis 2.

Hypothesis 2.1. Involvement in the online Autistic community will be positively related to self-determination.

Hypothesis 2.2. Communication preference will moderate the relationship between involvement in the online Autistic community and selfdetermination, with a more positive relationship between involvement in the online Autistic community and self-determination when a person prefers online communication over when a person does not prefer online communication.

Research Question Three

The third research question addressed the difference between communication preference moderating the relationship between involvement in the online community and self-determination in autistic adults and non-autistic adults. Specifically, the third research question asked: Does communication preference have a stronger moderating effect between involvement in the online Autistic community and self-determination than between involvement in the online community and self-determination in nonautistic adults? I predicted that the moderation of communication will be stronger in 
autistic adults than non-autistic adults $(\mathrm{H} 3)$. See Figure 4 for a representation of Hypothesis 3.

Hypothesis 3. Communication preference will have a stronger moderating effect on the relationship between involvement in the online community and self-determination in autistic adults than non-autistic adults.

\section{Research Question Four}

The fourth research question addressed the relationships among involvement in the online Autistic community, Autistic identity and self-determination in autistic individuals, and whether these relationships differ between individuals who prefer online communication to autistic adults who do not prefer online communication. Specifically, the fourth research question asked: How are involvement in the online Autistic community, Autistic identity, and self-determination (autonomy, competence, and relatedness) related among autistic individuals, and do these relationship differ between individuals who prefer online communication compared to autistic individuals who do not prefer online communication? As depicted in the conceptual model for the study (Figure 5), I predicted that the structural equation model is a plausible representation of the relationship among the constructs. I predicted that the Autistic Identity Scale is a plausible representation of the dimensions of Autistic identity adapted from the disability identity theory of Gill (1997), including the factors of integration into society, integration into the Autistic community, internal integration of similarities and differences with others, and integration of internal feelings of self with presentation of self ( $H$ 4.1). I also predicted that involvement in the online Autistic community will be 
positively related to Autistic identity in autistic adults who prefer online communication (H 4.2) and do not prefer online communication (H 4.3), involvement in the online Autistic community will be positively related to self-determination in autistic adults who prefer online communication (H 4.4) and do not prefer online communication (H 4.5), and Autistic identity will be positively related to self-determination in autistic adults who prefer online communication $(\mathrm{H} 4.6)$ and do not prefer online communication $(\mathrm{H}$ 4.7). I also predict that the relationships among the variables in the predicted structural model will be stronger in autistic adults who prefer online communication $(\mathrm{H} 4.8)$.

Hypothesis 4.1. The Autistic Identity Scale containing the factors of integration into society, integration into the Autistic community, internal integration of similarities and differences with others, and integration of internal feelings of self with presentation of self is a plausible representation of the dimensions of Autistic identity adapted from the disability identity theory of Gill (1997). Specifically, the scale and subscales will exhibit acceptable internal consistency reliability (Cronbach's alpha of .70), and the four factors of the Autistic Identity Scale will have a positive significant regression weights on Autistic identity in the proposed structural model.

Hypothesis 4.2. Involvement in the online Autistic community will be positively related to Autistic identity in autistic adults who prefer online communication. 
Hypothesis 4.3. Involvement in the online Autistic community will be positively related to self-determination in autistic adults who prefer online communication.

Hypothesis 4.4. Autistic identity will be positively related to self-determination in autistic adults who prefer online communication.

Hypothesis 4.5. Involvement in the online Autistic community will be positively related to Autistic identity in autistic adults who do not prefer online communication.

Hypothesis 4.6. Involvement in the online Autistic community will be positively related to self-determination in autistic adults who do not prefer online communication.

Hypothesis 4.7. Autistic identity will be positively related to self-determination in autistic adults who do not prefer online communication.

Hypothesis 4.8. The relationships among involvement in the online Autistic community, Autistic identity, and self-determination will be stronger in autistic adults who prefer online communication than in autistic adults who do not prefer online communication. 
CHAPTER IV

Methodology

This study examined the relationship between involvement in the online Autistic community and Autistic identity and involvement in the online Autistic community and self-determination, and whether communication preference moderates the relationships. It also compared the strength of the communication preferences moderation on involvement in the online community and self-determination in autistic adults and non-autistic adults. Finally, this study examined the relationships among involvement in the online Autistic community, Autistic identity, and self-determination in autistic individuals who prefer to communicate online and autistic individuals who do not prefer to communicate online. It utilized a cross-sectional online survey design. As indicated, the Well-Being Study adhered to a CBPR approach, and the current study partnered with AASPIRE for a community-engaged approach. Members collaborated using their strengths to develop and implement the overarching study, then provided me with feedback and approval on the steps of the current study.

\section{Research Context}

The Autistic self-advocacy movement as well as CBPR principles call for research conducted with autistic individuals aimed at improving lives of all autistic individuals (Robertson, 2010). To that end, the Gateway Project, the collaboration between AASPIRE and Dr. Morton Gernsbacher's Lab at University of Wisconsin-Madison, served 
as a gateway to research that is committed to four values, including respect, accessibility, inclusion, and relevance to the Autistic adult community. The purpose of the Gateway Project was to identify eligible participants, recruit them for continuing online studies, and collect data on variables that informed subsequent studies. The Gateway Survey collected data on variables used in the current study, including Internet and computer use, demographic information, disability and Autistic identity and diagnoses, and autistic traits. After participating in the Gateway Survey, AASPIRE used the demographic data and the data on diagnoses to identify eligible participants for AASPIRE's continuing projects, including the Internet Use, Community, and Well-Being Study. The present study utilizes data from the Gateway Survey and the Internet Use, Community, and Well-Being Study.

\section{Study Development}

The AASPIRE team first identified the importance of examining involvement in the online Autistic community. The AASPIRE team nominated the constructs of involvement in the online Autistic community, Autistic identity, sense of community, social support, and psychological well-being to be included in the study. We then developed all procedures and materials for the study as a team consisting of the academic principal investigator, Dr. Katherine McDonald, the community principal investigator, Dora Raymaker, and the academic co-investigator Dr. Christina Nicolaidis. The principal investigators and the AASPIRE team reviewed the procedures and materials. We conduct online meetings via Skype instant messaging, email, or in person 
to discuss any concerns or ideas team members wish to share. We make revisions in accordance to the group's discussions, and the whole AASPIRE team reviews a next draft once again.

This system of constant communication and checking in with all research partners results in much revision of procedures and materials to create understanding and relevance among the whole team. The researchers provide support by ensuring the quality of the design and measures, while the autistic partners ensure respectfulness, relevance, and clarity of procedures and measures. The principal investigators and coinvestigators made minor or time sensitive decisions.

We made decisions around choice of instruments and items by presenting options to the team and educating community partners on the reliability and validity of existing options. The team chose the Sense of Community Index 2, Autistic Identity Scale, Social Provisions Scale, and three of the more relevant Psychological Well-Being Scales based on theoretical appropriateness, accessibility, and face validity. The community partners approved usage of the scales overall, but required definitions or word changes for the word "community" and the different types of Internet uses. Community partners requested changes to make survey wording more clear and understandable, since "community" and types of Internet use (e.g., listserv) may be unclear to some individuals.

In order to retain the scientific validity of the psychometrically sound instruments while allowing for optimal comprehension, the AASPIRE research team utilized hotlinks for clarifying words or phrases within items. The AASPIRE research team 
worked extensively to create the most comprehendible hotlinks to supplement the original items if the participant requires clarification. The process of modifying survey items and hotlinks was utilized when community partners indicated that some of the items contained vague or confusing words or phrases. During team meetings, we identified vague or confusing aspects of individual items. We then discussed what words, phrases, or definitions would make the item comprehendible to our study population. We reached consensus on every item during team meetings. After team meetings, the investigators created hotlinks with the decided upon language. The team was asked to review the survey again with the hotlink changes and provide feedback in the next team meeting. This process was repeated until consensus was reached on the comprehension of all survey items.

\section{Measures}

The current study utilized five measures that are described below. These include the Gateway Survey, and the four measures from the AASPIRE Internet Use, Community, and Well-Being Study, including Internet Usage, Sense of Community Index 2, Autistic Identity Scale, and Self-Determination. See Figure 6 for a visual representation of the participants and measures utilized in the Gateway Project and the AASPIRE Internet Use, Community and Well-Being Study.

Gateway Survey. The Gateway Survey asked about Internet and computer use, demographic information, autistic identity/ diagnoses, hand preference, interests, habits, social interactions, and future research. This survey contained eight items that 
the current study used that asked about demographics, including age, gender, race/ ethnicity, income, education, parent education, and employment.

This study used five questions that specifically ask about autism spectrum diagnoses. These autism-specific questions include one item used as an inclusion criterion in the autistic sample of the study. This item is: "I identify as a person on the autistic spectrum (including autistic disorder, Asperger's, and PDDNOS), either because I have been formally diagnosed or because I have recognized that the criteria fit me" Participants must have indicated that they identify as on the autistic spectrum to be included in the autistic sample of the present study. All individuals who indicated that they do not identify as a person on the autistic spectrum were included in the nonautistic sample of the study. Some individuals identify as autistic without a professional diagnosis (Jordan, 2010), and many factors contribute to individuals not receiving a professional diagnosis, including the DSM-IV diagnostic criteria in use at the time of data collection (Grinker, 2007; Wing \& Potter, 2002) and socio-cultural factors. Since a number of studies indicate that autism spectrum disorders may be under-diagnosed (Brugha et al., 2011; Y. S. Kim et al., 2011), this study included all individuals who identify as autistic in the autistic sample.

The Autism-Spectrum Quotient (AQ; Baron-Cohen, Wheelwright, Skinner, Martin, \& Clubley, 2001) is a 50-item instrument measuring the degree to which an adult has traits associated with the autistic spectrum. If Gateway Survey participants indicated that they identify as autistic, they were asked the 50 items of the AQ. Individuals who did not identify as autistic were not asked the AQ items. The AQ 
assesses five different areas that represent autistic traits. These areas are social skill (e.g., "I prefer to do things with others rather than on my own"), attention switching (e.g., "I prefer to do things the same way over and over again"), attention to detail (e.g., "I often notice small sounds with others do not"), communication (e.g., "Other people frequently tell me that what I've said is impolite, even though I think it is polite"), and imagination (e.g., "If I try to imagine something, I find it very easy to create a picture in my mind"). Ten items asked about each of these areas. The items were completed on a scale response of "definitely agree", "slightly agree", "slightly disagree", and "definitely disagree". Positively scored items indicate that a response of "definitely agree" or "slightly agree" score one point on the AQ rating. Negatively scored items indicate that a response of "definitely disagree" or "slightly disagree" score one point on the AQ rating. A total $A Q$ score is derived from the sum of all items.

The cut-off score of 32 indicates that a person scoring 32 or higher has indicators consistent with clinically diagnosed ASD. The AQ has been shown to be a reliable measure (overall alpha .71 and .81 in two samples) as well as good screening properties when compared to clinical diagnostic criteria (Hoekstra, Bartels, Cath, \& Boomsma, 2008). The AQ also shows good reliability in the autistic sample of the current study with an overall alpha of .87. Studies have shown good discriminative validity and screening properties for the AQ using a cut-off of 26 or higher (Woodbury-Smith, Robinson, Wheelwright, \& Baron-Cohen, 2005). While the inclusion criteria for the current study was self-identifying as being on the autistic spectrum, AQ cutoff scores were included for descriptive purposes and to compare differences between participants who have 
autistic traits and do not have autistic traits (see Table 2 for participant demographics). In the analysis of the subset of current study sample, I found no significant differences between individuals at the cut-off of 26 and individuals at the cut-off of 32 (Kidney, 2012). While significance tests were performed in the complete sample of this study, Table 3 displays data with the cut-off of 32 to demonstrate the more conservative cutoff for inclusion in the group with indicators consistent with clinical diagnosis of ASD.

Finally, three questions with multiple choice responses asked about education and seven questions asked about employment and income. Two questions on education ("The highest level of education that I have obtained is:" and "The highest level of education either of my parents obtained is:") and four items on employment ("My employment status is:") and income ("During the past 12 months, my main sources of income were:") were used in the current study.

Internet Use, Community, and Well-Being Study. Four sections of the Internet Use, Community, and Well-Being Survey were used in this study. These four sections are Internet Usage, Autistic identity, Sense of Community, and Psychological Well-Being. I describe here the variables that were utilized in this study, as well as the sections from the survey that contain the variables. Respondents were asked questions depending on whether they identified as autistic or non-autistic with disabilities or without disabilities. Relevant to the current study, the Internet Usage section and the instructions for the Sense of Community section differed depending on the autistic and disability identity of the respondent. The following descriptions of the measure include the specific differences among the survey depending on the participants' identity. 
Involvement in the online community. The measurement of involvement in the online community compromises of four dimensions, including (1) frequency of involvement in the online community, (2) years of involvement in the online community, (3) sense of community in the online community, and (4) importance of involvement in the online community. A review of the literature (see Chapter III) and community input contributed to the selection of the dimensions that were included in the measurement of involvement in the online community. However, there are contradicting opinions about the utility of individual dimensions and the weighting of each dimension. Therefore, a data-driven approach compliments the theoretical and community-driven decision to include the four dimensions by determining specific weights of each dimension in the composite measurement. Principle components analysis determined the data-driven weight of each dimension included in the variable, with the principal component loading of each domain used to compromise a weight (Njong \& Ningaye, 2008). In the Internet Use, Community, and Well-Being Survey, the Internet Usage section asked about participants' computer use, Internet access, involvement in any online communities, and involvement specifically in the online Autistic community. The survey asked non-autistic adults with no disabilities to respond to items on computer use, Internet access, and involvement in online communities they regularly use to communicate with others and share common interests, goals, activities, and/or identity. The survey asked non-autistic adults with disabilities to respond to the same items specifically in the online disability community. 
The Internet Usage section contained three of the dimensions of involvement in the online community in autistic and non-autistic individuals, including frequency, years, and importance of involvement in the online community. The AASPIRE team developed the Internet Usage measure for the Internet Use, Community, and Well-Being Study. Items for this section were modeled from similar items in the Gateway Survey and Gallup Poll (Gallup, 2009), and then modified by the AASPIRE research team with input from the community partners on comprehension of the language. The remaining dimension of sense of community were from the Sense of Community section of the survey, which utilized the Sense of Community Index 2 (Chavis et al., 2008).

In the autistic sample, the study measured involvement in the online Autistic community through an item asking, "In the past six months, I have used the Internet to socialize, interact, exchange information, coordinate events, or participate in any activity that involved interacting with other autistic people over the Internet:" In the non-autistic sample, the study measured involvement in the online community for nonautistic individuals though a similar item, except the word "autistic" is removed, making the item end with "...interacting with other people over the Internet:". Both in the autistic sample item and non-autistic sample item, the item response options were: never, less than once a month, at least once a month but less than once a week, at least once a week but less than once a day, less than one hour daily, 1-3 hours daily, 3-6 hours daily, or more than 6 hours daily. The option for "cannot or do not wish to say" was also included, which is coded as missing data for analyses. In order to create a continuous composite variable containing a weighted value of this variable, the variable 
of frequency of involvement is transformed into a continuous variable, numbered from 0 to 7. Despite the categorical measurement of frequency of involvement, the underlying latent variable of time lends itself to such a continuous coding (Njong \& Ningaye, 2008). Therefore, a response of "never" is changed to 0, "less than once a month" is 1, "at least once a month but less than once a week" is 2, "at least once a week but less than once a day," is 3, "less than one hour daily" is 4, "1-3 hours daily" is 5, "3-6 hours daily" is 6 , and "more than 6 hours daily" is 7, with the scale assumed to be continuous for the purpose of the study analyses.

The item that measured years of involvement in the online Autistic community asked the participants, "I have been participating in any sort of online Autistic community since:" with a dropdown menu with each year from 1972 to 2011 . The item that measured years of involvement in an online community for non-autistic individuals asked the participants, "I have been participating in any sort of online community since:" with the same dropdown menu with each year from 1972 to 2011 . The next variable that utilized in the involvement in the online community composite variable asked about the importance and value of involvement in the online Autistic community ("I consider my involvement with online Autistic communities to be an important and valuable part of my life"), with a Likert scale response option. For importance of involvement in any online community for non-autistic individuals, this item asked: "I consider my involvement with any online communities to be an important and valuable part of my life" with the same Likert scale response option. The AASPIRE team developed the variables for importance of involvement in the online Autistic community 
and any online community variables to tap into the subjective, perceived value of involvement in the community in order to mirror findings from previous research.

The remaining domain of involvement in the online community was sense of community in the online community. The Sense of Community Index version $2(\mathrm{SCl}-2)$ is the second version of the original $\mathrm{SCl}$ that contains 25 items that measure a person's sense of community (Chavis et al., 2008). The first item ("How important is it to you to feel a sense of community with other community members?") is a validating question that has a strong positive correlation with the total sense of community score (Chavis et al., 2008). Since the purpose of this item is for scale validation and not analysis, this item was not used in the current analysis. However, the current study utilized three of the four subscales, compromising 18 of the items. These items contain statements that represent how the participant feels about the community. For example, "I can trust people in this community". The survey asked autistic participants to think of the online Autistic community that they feel most involved when responding to the items, which was described in the section instructions as "comprised of primarily people on the autism spectrum (such as WrongPlanet...), or may have a common purpose that is specifically related to Autistic advocacy (such as the autistic Self Advocacy Network yahoo group...)." The survey asked participants who did not identify as autistic or with disabilities to think of the online community that they feel most involved when responding to the items. The section instructions defined online communities as "groups of people who communicate with each other regularly over the Internet and share some common interests, goals, activities, and/or identities." The survey asked 
participants who identified as non-autistic with disabilities to think of the online disability community in which they feel most involved. The survey instructions described the online disability community as "composed primarily of people with disabilities or may have a common purpose that is specifically related to disability advocacy."

The scale is based on the theory of sense of community developed by McMillan and Chavis (1986). The three subscales of the SCl-2 correspond to the three dimensions of the theory of sense of community that I utilized for analyses. The subscale of membership contains six items including: "Being a part of this community is part of my identity." The subscale of influence contains six items including: "Fitting into this community is important to me." The subscale of integration and fulfillment of needs contains six items including: "Being a part of this community makes me feel good." Participants completed all items on a Likert scale response ranging from 0 (Not at All) to 3 (Completely). Subscale scores were the means of the respective items within each subscale. A total sense of community mean was derived from the mean of items 1 through 18, which were used for analyses. Responses to negatively scored items were reversed in the final scoring procedures so that high scores indicate high ratings on each dimension of sense of community. The coefficient alpha of the study sample of autistic adults showed excellent overall reliability (Cronbach, 1951) with an alpha of .91. The subscales of the study of autistic adults indicated the reliability of the membership subscale as good ( $\alpha=.81)$, the influence subscale was acceptable ( $\alpha=.73)$, and integration and fulfillment of needs was good $(\alpha=.85)$. The coefficient alpha of the study of nonautistic adults also showed excellent overall reliability (Cronbach, 1951) with an alpha of 
.94. The subscales of the study of non-autistic adults indicated the reliability of the membership subscale as good $(\alpha=.81)$, the influence subscale was good $(\alpha=.82)$, and integration and fulfillment of needs was excellent $(\alpha=.90)$.

Autistic Identity Scale. The AASPIRE research team adapted the Autistic Identity Scale from Gill's Disability Identity Scale (Gill, 1997b). Autistic participants in the sample completed the Autistic Identity Scale, and participants with disabilities completed the original Disability Identity Scale (Gill, 1997b). The Autistic Identity Scale was included in the current study, but the Disability Identity Scale was not utilized for the current study analyses. The Autistic Identity Scale includes 17 items containing statements about how the participant feels about being autistic. Gill's disability identity theory provided the basis for the scale (Gill, 1997a), but language was adapted to be autism specific (e.g., "I believe that because I am a person with a disability, I have many strengths" was modified to "I believe that because I am autistic, I have many strengths"). In addition to adapting the scale to be autism specific, the AASPIRE research team worked to adapt some of the scale language for clarity and appropriateness for the participants. This included changing the original phrase "my destiny" to "my future".

Despite its theoretical grounding, very little research has investigated the scale's psychometric properties. However, the items of the survey address each of the four dimensions of Gill's theory, though the specific assignment of items to each dimension is unknown. These dimensions are integration into society ("Overall, people on the autistic spectrum are considered valuable by others"), integration with the Autistic community ("I have a strong sense of belonging to the Autistic community"), internally integrating 
one's similarities and differences with others ("In general, others respect people on the autistic spectrum"), and integrating how one feels with how one presents oneself ("Being autistic is an important reflection of who I am"). Each item was completed on a Likert scale response ranging from 1 (Strongly Disagree) to 5 (Strongly Agree). The mean of all Likert scale responses results in a composite score for analysis use. I reverse scored responses to negatively scored items in the final scoring procedures so that high scores indicate high ratings on the dimension. The current sample indicated good reliability $(\alpha=.73)$.

Self-Determination. Ryan and Deci's self-determination theory (Deci \& Vansteenkiste, 2004; Ryan \& Deci, 2000) is comprised of three psychological needs that, in a supportive context, contribute to self-determination. The three psychological needs are autonomy, competence, and relatedness. This study created measures of the three domains via available measures and items in the AASPIRE Internet Use, Community, and Well-Being Study. The Ryff Scales of Psychological Well-Being measures six domains of psychological well-being: autonomy, environmental mastery, personal growth, positive relations with others, purpose in life, and self-acceptance. The self-determination domains of autonomy and competence were measured by scales in the Ryff Scales of Psychological Well-Being (Ryff, 1989), including autonomy and environmental mastery, respectively. As competence is effective control over an individual's environment (Deci \& Vansteenkiste, 2004), the items of the environmental mastery subscale are closely aligned with the theoretical basis for the concept of competence. Self-determination and motivation are theoretically discussed as closely related to psychological well-being 
(Huta \& Ryan, 2009; Ryan, Huta, \& Deci, 2006) and empirically relating to well-being, happiness, and self-actualization (Johnson \& Finney, 2010; Meyer et al., 2007;

Vansteenkiste et al., 2006). The parallel use of autonomy and environmental mastery, in addition to theoretical overlap justifies use of scales from Ryff's Psychological WellBeing.

Three forms of each of the Ryff Scales of Psychological Well-Being exist. These are the long form (14 items), medium form (9 items), and short form (3 items). The scales in short form have shown low internal consistency and are not used for the present study. No previous psychometric properties are available from previous studies utilizing the 9-item survey version (medium form), which was used for the present study because of considerations for respondent burden. The items utilize a 6-point Likert scale ranging from "Strongly disagree" to "Strongly agree." In the current study sample, the autonomy scale indicated good reliability (Cronbach, 1951) in the overall sample with an alpha of .81 (both autistic and non-autistic sample alphas are both .81 as well). Ryan, Huta, and Deci (2006) argue that self-determination theory conceptually focuses more on volition and self-endorsement than Ryff's (1989) dimension of psychological wellbeing that is more focused on independence of thought and action. The Basic Need Satisfaction Scale (BNSS) was developed in accordance with Ryan and Deci's (2000) selfdetermination theory (Gagne, 2003; Kashdan, Julian, Merritt, \& Uswatte, 2006). However, in a series of analyses aimed at examining the psychometric properties of the BNSS, Johnston and Finney (2010) found a very strong significant correlation between the BNSS autonomy subscale and Ryff's autonomy scale of psychological well-being 
$(r=.64, p<.05)$ indicating that the measurement of the two dimensions are very similar. Similarities between the two scales can also be seen on the item level. For example the items: "I generally feel free to express my ideas and opinions" (BNSS) and "I am not afraid to voice my opinions, even when they are in opposition to the opinions of most people" (Psychological Well-Being Scales) both tap into speaking up about personal feelings, "I feel pressured in my life" (BNSS) and "My decisions are not usually influenced by what everyone else is doing" (Psychological Well-Being Scales) both refer to outside influences, and "In my daily life, I frequently have to do what I am told," (BNSS) and "I often change my mind about decisions if my friends or family disagree" (Psychological Well-Being Scales) both refer to making ones' own decisions.

The competence scale indicated good reliability in the overall sample with an alpha of .88 (autistic sample $\alpha=.82$, non-autistic sample $\alpha=.89$ ). In addition to exhibiting good internal consistency, Helson and Srivastava (2001) found that Ryff's environmental mastery scale (utilized for the current study to measure competence) is a positive predictor of competence measured by the California Personality Index (Gough \& Bradley, 1996). In examining the psychometric properties of the BNSS, Johnston and Finney (2010) found an extremely high correlation between the competence scale of the BNSS and Ryff's Psychological Well-Being Scale of competence at $r=.82, p<.05$, indicating that the current study's measure of competence is closely aligned with both measures of competence. Items from the scales also closely align with one another, for example "Often I do not feel very competent" (BNSS) aligns with "In general, I feel I am in charge of the situation in which I live" (Psychological Well-Being Scales) and "Most days I feel a 
sense of accomplishment from what I do" (BNSS) aligns with "I am quite good at managing the many responsibilities of my daily life" (Psychological Well-Being Scales).

The third psychological need of self-determination theory is relatedness, or the need to meaningfully connect with others (Deci \& Vansteenkiste, 2004). Ryff's Psychological Well-Being Scales (1989) include a measurement of positive relations with others that may be appropriate for the measure of relatedness in the current study. However, this scale was not included in the Well-Being Study. Instead, the AASPIRE Internet Use, Community, and Well-Being Study utilized the $\mathrm{SCl}-2$ as a measure of SOC, which contains a subscale of shared emotional connection (Chavis et al., 2008). Shared emotional connection is having close relationships with members of the community and a sense of shared history and understanding (McMillan \& Chavis, 1986). Due to the strong similarity between the dimension of shared emotional connection and the concept of relatedness, the current study utilized the 6 items measured in this dimension as a measurement of relatedness. The items each utilize a 4-point Likert scale ranging from "Do not agree at all" to "Completely agree" This relatedness subscale indicated good reliability (Cronbach, 1951) with an alpha of .87 in the overall study sample (autistic sample $\alpha=.86$, non-autistic sample $\alpha=.88$ ). No previous studies have empirically examined the correlation between measures of self-determination and the $\mathrm{SCl}-2$. However, the items of the $\mathrm{SCl}-2$ shared emotional connection subscale align closely with the items in the BNSS relatedness subscale. Examples of the close items include: "I really like the people I interact with" (BNSS) and "I am with other community 
members a lot and enjoy being with them" (SCI-2), and "People in my life care about me" (BNSS) and "Members of this community care about each other" (SCl-2).

In order to combine the three subscales for the overall measurement of selfdetermination, I aligned the Likert response scales across the subscales. I conducted a transformation on the 6-point scales of the autonomy and competence subscales to make them equivalent to the 4-point scale of the relatedness scale. The overall measurement of self-determination, including autonomy, competence, and relatedness, indicated very good reliability (Cronbach, 1951) in the current sample with an overall alpha of .91 (autistic sample $\alpha=.88$, non-autistic sample $\alpha=.91$ ).

\section{Participants}

The present study utilized data from the autistic and non-autistic participants in the AASPIRE Internet Use, Community, and Well-Being Study. To be eligible for participation in the Gateway Project, participants must have had access to the Internet, be over the age of 18, and be able to read in English. However, participants completed the survey worldwide. To be eligible for participation in the AASPIRE Internet Use, Community, and Well-Being Study, participants must have completed the Gateway Survey. Gateway Survey participants who indicated they would like to receive extra credit in a course at the University of Wisconsin-Madison were ineligible for participation in order to ensure a more diverse sample compromised of individuals from a variety of ages, socioeconomic backgrounds, and geographic locations. 
In addition to indicating that they were not a student seeking extra credit, the current study based eligibility on the participants' responses to two items concerning autism and disability in the Gateway Survey. The first item asked: "I consider myself to be on the autistic spectrum (including autistic Disorder, Asperger's disorder, and PDDNOS): Yes, and I have been formally diagnosed; Yes, but I have not been formally diagnosed; No." From the Gateway Project participants, the study invited a random selection of eligible participants that self-identify as autistic by considering themselves autistic (regardless of diagnosis) to participate as a member of the sample of autistic individuals in the AASPIRE Internet Use, Community, and Well-Being Study. The study also invited non-autistic individuals with disabilities from the Gateway Project participants who responded with "No" on the autistic spectrum question, and then "Yes" to the Gateway Survey item that asked: "I consider myself to have a disability." After autistic adults began the AASPIRE Internet Use, Community, and Well-Being Study, the study invited those that do not self-identify with a disability or autism to participate by a matching process. The study randomly selected non-autistic individuals without a disability from matches to autistic participants based on gender and age groups to ensure comparability on basic demographic characteristics. The inclusion criteria for both the autistic and the non-autistic samples in the current study included individuals who indicate they consider themselves to have disabilities.

Since the inclusion criteria for the autistic sample only asked if participants consider themselves to be on the autism spectrum regardless of whether they have an autism diagnosis, the sample included any participants who have not been 
professionally diagnosed with ASD but who indicated on the item in the Gateway Survey that they identified as on the autistic spectrum. The AASPIRE team decided on the inclusion criteria (identifying as autistic) for a number of reasons. Some individuals identify as autistic without a professional diagnosis (Jordan, 2010). The AQ and other screening and diagnostic tools have been shown to have a gender bias, leading to females being under-diagnosed and improperly screened out of autism research (Y.S. Kim et al., 2011; Lord \& Schopler, 1985), as well a theory of autism being an "extreme male brain" (Baron-Cohen, 2002). In addition to problems of screening and diagnostic tools, many other factors contribute to individuals not receiving a professional diagnosis, including the DSM-IV changes to pervasive developmental disorder diagnoses creating a disparity in diagnoses among older adults who received a diagnosis inconsistent with current PDD or no diagnosis at all (Wing \& Potter, 2002), as well as sociocultural factors (e.g., poverty, stigma, lack of education). Population-based studies indicate that autism spectrum disorders may be underdiagnosed (Brugha et al., 2011; Y. S. Kim et al., 2011). Therefore, the inclusion criteria for the sample allowed for a broader sample that may be more representative of autistic adults than excluding based on diagnosis or AQ score.

The Gateway Survey did not collect IP addresses of identifying information in order to protect the confidentiality of the data. Therefore, once the inclusion criteria deemed cases in the Gateway Survey eligible for participation in the AASPIRE Internet Use, Community, and Well-Being Study based on not seeking extra credit for a course and indicating whether they identified as having a disability, being autistic, or not having 
a disability or being autistic, we examined the validity of the data. The Gateway Council and AASPIRE identified patterns of invalid cases in the Gateway Survey, including consistently mismatching information on responses for sex and gender, and open-ended responses that were not relevant to the item question or that contain strings of words without meaning. Based on these criteria, we deemed cases ineligible for participation in the AASPIRE Internet Use, Community, and Well-Being Study if their sex and gender did not match within the Gateway Survey. Though individuals may identify with stereotypically non-matching sex and gender categories, we created a systematic method of highlighting cases with numerous indicators of invalid Gateway dataincluding but not limited to non-matching sex and gender categories-- which we closely examined before removal from the sampling frame of the current study.

The sample of 324 eligible individuals who completed the AASPIRE Internet Use, Community, and Well-Being Study included 151 autistic participants and 173 nonautistic participants. Autistic participants ranged in age from 18 to $70(M=36.96$, $S D=12.32)$, and non-autistic participants ranged in age from 18 to $74(M=37.92$, $S D=13.10$ ). The majority of participants identified as White (autistic sample $=88.7 \%$, non-autistic sample=83.8\%). Participants who identified as Latino or Hispanic make up $4.0 \%$ of the autistic participants and $4.6 \%$ of the non-autistic participants. Of the 151 autistic participants, $40.4 \%$ were male, $51.0 \%$ were female, $1.3 \%$ was transgender, and 7.3\% were intersex. Of the 173 non-autistic participants, $38.2 \%$ were male, $60.1 \%$ were female, $0.6 \%$ was transgender, and $0.6 \%$ was intersex. Over $90 \%$ of the autistic and the non-autistic samples obtained education past a high school diploma or GED. The survey 
also asked participants about the highest level of education one or both of their parents achieved, indicating that approximately $75 \%$ of autistic and non-autistic participants had at least one parent who obtained education past a high school diploma or GED. See Table 2 for detailed information on age, ethnicity, gender, education level, and parent education level. Demographic variables related to the presence of autistic traits and autism spectrum disorder diagnoses in the autistic sample are presented as frequencies and percentages of the sample in Table 3.

Of the 151 participants who identified as autistic, $72.8 \%$ indicated the diagnosis that fits them best is Asperger's disorder, 20.5\% indicated autistic disorder, $0.7 \%$ indicated Rett's disorder, $2.0 \%$ identified as pervasive developmental disorder- not otherwise specified, $1.3 \%$ wrote in "on the autism spectrum," and $2.6 \%$ did not know or wish to say. When the survey asked about formal diagnoses, $67.5 \%$ of participants indicated they had a formal diagnosis with an autism spectrum disorder and $32.5 \%$ indicated they did not have a formal diagnosis with an autism spectrum disorder.

Also, $81.5 \%$ of participants scored a 32 or higher in the Autism-Spectrum Quotient, indicating characteristics consistent with clinically diagnosed ASD (S BaronCohen et al., 2001). Using the less conservative AQ cut-off score of 26 that is recommended in current clinical screening measurements (Woodbury-Smith et al., 2005), the sample consisted of $91.4 \%$ participants with characteristics consistent with clinically diagnosed ASD. The sample contained 8.6\% (26 AQ cut-off) to 17.9\% (32 AQ cut-off) individuals who do not meet the AQ cut-off scores but identify as autistic. 
Procedure

Starting on June 25, 2009, AASPIRE recruited eligible participants for the Gateway Project via autism-related and disability-related organizations, websites, list serves, and blogs as well as through postings on campus at the three participating universities and word of mouth. AASPIRE used flyers and announcements to target three groups for participation in the Gateway Project: autistic adults, non-autistic adults with disabilities, and non-autistic adults with no disabilities. In addition to online recruitment through autism-related and disability-related venues, we specifically targeted non-autistic participants via Craigslist and Facebook, but no communityspecific venues. To register for the Gateway Project and complete the Gateway Survey, the participant entered an email address and creates a password. This allowed the participant to start the survey and returned to it later if incomplete (and so that data from different studies can be linked together). After participants entered a username and password, the website directed participants to the Information Sheet, which was a page containing a description of the Gateway Project. This description included the purpose of the study, details about the types of questions asked in the survey, and provided the benefits and minimal risks of participating. The participant indicated consent after the participant read this information and continued to the next page. The following pages contained measures of computer and Internet use, demographic information, disability and autistic identity and diagnoses, hand preferences, presence of autistic traits, and future research. The survey took approximately $20-40$ minutes to 
complete. Participants were not compensated for participating in the study, although students at the University of Wisconsin-Madison were compensated with an extra credit point for participating in the Gateway Survey. However, if participants completed the study, they chose to be entered into a drawing for a \$25 gift certificate to Amazon.com. A winner was chosen randomly after each group of 25 participants completed the survey, so 1 in 25 participants received the gift card.

When the AASPIRE Internet Use, Community, and Well-Being Study launched data collection on February 24, 2011, AASPIRE also disseminated recruitment messages about the Gateway Project and the new study (i.e., the AASPIRE Internet Use, Community, and Well-Being Study) over listservs and posted in diverse communities with permission from moderators, directing those who were not yet registered with the Gateway Project to register and complete the Gateway Survey. AASPIRE utilized many of the same organizations, websites, list serves, and blogs for continued recruitment for the Gateway Project, including targeting autistic and disability venues.

AASPIRE recruited for the AASPIRE Internet Use, Community, and Well-Being Study occurred with those who participated in the Gateway Project, completed the Gateway Survey and met eligibility criteria (i.e., completed the Gateway Survey, did not indicate they would like to receive extra credit in a course at the University of Wisconsin-Madison, and met validity check criteria of matching sex and gender). AASPIRE sent eligible individuals up to three notifications of the AASPIRE Internet Use, Community, and Well-Being Study lead by Dr. Katherine McDonald and Dora Raymaker. The message linked participants to a personal study webpage (which required 
participants to login with their Gateway Project login and password) with information on the AASPIRE Internet Use, Community, and Well-Being Study.

After the username and password were entered, the website directed the participant to a page containing a description of the study. The description of the study included the purpose of the study, details about the types of questions asked in the survey, and provided the benefits and minimal risks of participating. The participant indicated consent after the participant read this information and continued to the next page. The following pages contained measures of Internet use, psychological well-being, Autistic identity, sense of community, online social support, face-to-face social support, and demographic information. The survey took approximately 30-45 minutes to complete.

The study did not compensate participants for participating in the AASPIRE Internet Use, Community, and Well-Being Study. However, after participants completed the study, they could choose to be entered into a drawing for a \$25 gift certificate to Amazon.com. A winner was chosen randomly after each group of 15 participants completed the survey, so 1 in 15 participants receive a gift card. The survey was open for participation and data was collected for the present study for approximately 13 months. For the current study, we collected data from 151 eligible autistic individuals and 173 eligible non-autistic individuals between February 24, 2011 and April 22, 2012. In order to protect the confidentiality of the data, we did not collect IP addresses or identifying information such as names or email addresses. To ensure the validity of the data, we subjected cases to date-validation procedures prior to including otherwise 
eligible cases in the current study. For a case to be included in the current study, we examined whether the value for date of birth in the Gateway Survey matched the value for date of birth in the AASPIRE Internet Use, Community, and Well-Being Study. We excluded cases if the date of birth of each case was mismatched by more than two months and more than two years between the Gateway Survey and the AASPIRE Internet Use, Community, and Well-Being Study. We subjected cases still included to further scrutiny of matching demographic variables between the Gateway Survey and the AASPIRE Internet Use, Community, and Well-Being Study. These variables included matching gender and race/ethnicity responses in both surveys. Next we examined openended responses. Invalid cases in the Gateway Survey conformed to specific patterns, including within the responses to open-ended items. The invalid cases included openended responses not relevant to the question or containing strings of words without meaning. Therefore, if a case's gender or race/ethnicity did not match between the two studies, we carefully examined the individual case's open-ended responses to determine whether or not a case was valid. We deemed a case ineligible after demographics did not match and the open-ended responses conformed to the patterns among fake accounts that we identified. As a result of the validity check on the cases, 9 out of the 333 cases are excluded from the analysis. The total number of eligible cases I utilize in the study was 324 cases, including 151 autistic participants and 173 non-autistic participants.

For the current study, we collected data from 324 eligible individuals. Information on individuals' decision to actively or passively decline participation was not 
stored. However, out of the 492 email invitations sent to eligible autistic individuals to participate in the AASPIRE Internet Use, Community, and Well-Being Study, 11 emails bounced and therefore were never received, 7 individuals started the survey but did not complete the survey, 82 completed the survey but were later deemed ineligible for participation, and 259 were invited but did not begin the survey. The response rate of eligible autistic individuals who were invited to the survey without a bounced email and completed the survey is $37.84 \%$. Out of the 763 email invitations sent to eligible nonautistic individuals to participate in the AASPIRE Internet Use, Community, and WellBeing Study, 19 emailed bounced, 7 individuals started the survey but did not complete the survey, 82 completed the survey but were later deemed ineligible for participation, and 590 were invited but did not begin the survey. The response rate of eligible nonautistic individuals who were invited to the survey without a bounced email and completed the survey is $23.25 \%$. Over the entire sample, the response rate of eligible individuals invited to the survey without a bounced email and completed the survey was $28.35 \%$.

\section{Ethical Considerations}

Research involving online communities must make unique ethical considerations (Brownlow \& O'Dell, 2002). The present study also concerned research with a potentially vulnerable population of autistic adults. Here I address the risks and the associated safeguards of the study. 
There was a small risk in loss of confidentiality by sharing personal information. However, we protected participants' information by not recording any Internet Protocol (IP) addresses and using Secure Sockets Layer (SSL) encryption to make sure no one can see the information sent through the Internet. We store all information on passwordprotected computers to which only AASPIRE research team members and Gateway Project researchers have access. The study informed participants that they can ask the investigators to delete their information from the data file at any time by contacting them. Also, we used a code number to link the information provided to identifying information. Only the investigators are able to link the code numbers to identifying information. Finally, we use no identifying information in any publications, presentations, or reports.

In addition to confidentiality risks, participants may have become frustrated completing the survey if the computer or Internet were having problems. Also, there is a small risk that the participants might have become tired or found questions difficult or frustrating to answer. The online Information Sheet informed participants that they may exit the survey at anytime, with the ability to start where they left off if they wish to later.

AASPIRE made ethical considerations in accordance with the Portland State University Human Subjects Research Review Committee as well as the Oregon Health and Science University (OHSU), University of Wisconsin-Madison, and Syracuse University Institutional Review Boards. In an effort to not duplicate IRB review, PSU entered into an IRB authorization agreement with OHSU for the AASPIRE Internet Use, 
Community, and Well-Being Study. The IRB at University of Wisconsin-Madison and the IRB at OHSU provided approval for the Gateway Project. In accordance with the Office of Graduate Studies at Portland State University, the Human Subjects Research Review Committee reviewed the current dissertation study. Furthermore, by our close collaboration and use of community based participatory research methods for the overarching study and community-engagement with the current study, we bolstered the ethical issues related to the process of the project by ongoing conversations and actions around promoting the ethical issues relevant to respect, accessibility, inclusion, and relevance of the current study (Snow, Grady, \& Goyette-Ewing, 2000).

Due to the low risk nature of the study, the study modified the consent process to fit the constraints of online data collection. AASPIRE recruited participants through the Gateway Project and directed to the Gateway Survey before providing access to the present study. We invited eligible participants to participate in the AASPIRE Internet Use, Community, and Well-Being Study via email, and directed participants to the AASPIRE website and the AASPIRE project descriptions, which both described in detail the mission and purposes of the studies in a broader sense. This created a necessary level of transparency to the research process (Brownlow \& O'Dell, 2002). An online Information Sheet provided all the necessary information regarding purpose and procedures of the study, which the participants could have printed off if desired. After reading the Information Sheet, the survey website asked participants to check a box if they agree to proceed, after which the participant was granted access to the survey with consent assumed. This modified consent procedure was appropriate due to the minimal 
risk of the study. The AASPIRE team also concluded that the assessment of decisional capacity would be offensive to participants and not a necessary safeguard given our population of interest and the low risk nature of the research (Dye, Hendy, Hare, \& Burton, 2004). The team worked diligently to create materials that were clear and comprehendible to all participants able to understand the communication occurring in the websites, blogs, and listservs from which they were recruited. The Well-Being Study utilized the same method of informed consent procedures as the Gateway Project. The participants were directed to the project's description, which described the mission and purpose of AASPIRE and the current study. The online Information Sheet provided the necessary information regarding purpose and procedures, and finally, the participants were asked to check a box if they wish to proceed, indicating consent.

There were no direct benefits from participating in the study. However, participants may have felt satisfied by possibly benefiting persons with disabilities or persons on the autistic spectrum in the future. Also, participants may have felt satisfied from contributing to research and having their voice and perspective represented in science. 


\section{CHAPTER V}

Results

Descriptive Analyses. In order to describe the sample of participants, I conducted descriptive analyses after data cleaning using IBM SPSS 20. I calculated the mean and standard deviation of the demographic variables age. I calculated frequencies for the categorical demographic variables income, gender, race/ ethnicity, employment, and parent employment. Results of the descriptive analyses are in Table 2 and described in the Participants section of Chapter IV. The overall sample mean age was 37.4 years $(S D=12.75)$. Most of the sample identified as white $(86.1 \%)$ and female $(55.9 \%)$ with a personal annual gross income $\$ 24,999$ or less $(50.3 \%)$ despite the education level of $25.0 \%$ of the sample having a bachelor's degree or equivalent and another $20.4 \%$ having taken some college or university courses. Additionally, employed full-time at a regular job was the most common employment status in the sample at $37.3 \%$.

Next, I conducted descriptive analyses with the four dimensions of the involvement in the online community composite variable. I calculated means for years involved in the online community (autistic adults $M=5.25, S D=3.66$; non-autistic adults $M=9.93, S D=5.85$ ), importance of involvement in the online community (autistic adults $M=5.58, S D=1.48$; non-autistic adults $M=4.86, S D=1.58)$, and sense of community (autistic adults $M=2.28, S D=0.55$; non-autistic adults $M=2.19, S D=0.60$ ). $I$ calculated frequencies for frequency of involvement in the online community, with the highest 
number of individuals spending 1 to 3 hours daily online (33.10\% of autistic adults, $38.73 \%$ of non-autistic adults). See Table 4 for the results of the descriptive analyses. I calculated frequencies for what activities individuals participate in during their time online. Among autistic individuals who responded to the question ( $n=142), 116$ individuals used forum, blog, wiki, or other community-edited web page (81.69\%), 116 individuals used social networking sites (81.69\%), 13 used listservs or newsgroups (9.15\%), 44 used live chat groups (30.99\%), and 13 used virtual environments (9.15\%). Among non-autistic individuals who responded to the question ( $n=165), 96$ used forum, blog, wiki, or other community-edited web page (58.18\%), 137 used social networking sites (83.03\%), 12 used listservs or newsgroups (7.27\%), 30 used live chat groups (18.18\%), and 13 used virtual environments (7.88\%).

I also calculated frequencies for the construct of communication preference. Among autistic individuals, 74 participants (49.0\%) preferred online communication and 71 participants $(47.0 \%)$ did not prefer online communication (as indicated by preferring face-to-face communication or having no preference). Among non-autistic individuals, 53 participants (27.2\%) preferred online communication and 140 participants (71.8\%) did not prefer online communication. I calculated a mean for Autistic identity in the autistic sample $(M=4.00, S D=.63)$ prior to conducting an exploratory factor analysis and scale modifications.

Lastly, I calculated a mean for the construct of self-determination in the overall sample $(M=2.75, S D=.40)$ as well as the subsets of the sample including autistic individuals $(M=2.64, S D=.76)$ and non-autistic individuals $(M=2.84, S D=.38)$. I calculated 
subscale means for the self-determination measure subscales of autonomy (overall sample $M=3.09, S D=.48$; autistic sample $M=3.03, S D=.51$; non-autistic sample $M=3.14$, $S D=.46$ ), competence (overall sample $M=2.67, S D=.65$; autistic sample $M=2.36, S D=.56$; non-autistic sample $M=2.91, S D=.62$ ), and relatedness (overall sample $M=2.43, S D=.72$; autistic sample $M=2.49, S D=.76$; non-autistic sample $M=2.39, S D=.70)$. I conducted further descriptive analyses on the scales in the two samples in order to determine whether age or income should be included as control variables in hypotheses testing. I selected age as a potential control variable due to literature formerly reporting that age disparities existed among individuals with disabilities and autistic individuals that use the Internet (Cromby \& Standon, 1999). Further, literature suggests differences in Internet use among different income groups (Gallup, 2009). However, no significant age or income differences existed between the autistic and nonautistic individuals or between individuals who preferred online communication and individuals who did not prefer online communication. Other demographics in the sample such as gender and education were similar across the autistic and non-autistic sample as well, so no analyses in this study utilized control variables. While demographic differences may exist among all types of Internet use, perhaps involvement in online communities or participation in online surveys draws a more homogenous demographic population, which is also supported in literature finding similarities between autistic adult Internet users and the general population (Mazurek, 2013). 
Screen for Outliers, Normality, Linearity, and Multicollinearity. Prior to any

analyses, I screened data for univariate and multivariate outliers. I examined histograms for the univariate normality of the variables. I screened for multivariate outliers by checking Mahalanobis distance. The structural equation model assumed multivariate normality, so I also performed a test of the multivariate coefficient of kurtosis. In examining the involvement in the online community variable, the dimension of years involved in the online community contained a number of outliers and was skewed and showed different variances between populations on the variable. I applied a square root transformation to the variable (Tabachnick \& Fidell, 1996), which served to eliminate issues of skewness, kurtosis, and outliers. All further analyses used the transformed variable. No other variables showed issues of non-normality. I randomly examined a number of pairwise scatterplots to assess the linearity of observed variables, as structural equation modeling assumes linearity as well. None of the pairwise scatterplots showed issues of non-linearity. I tested multicollinearity with the variance inflation factor (VIF) in each of the analyses to indicate whether a redundant variable was included in each analysis (Kline, 1998). The tolerance and VIF statistics indicated no multicollinearity issues with the involvement in the online community, communication preference, self-determination, or Autistic identity variables. The tolerance was over .40 in all analyses and the VIF was under 2.5 in all analyses, which are within acceptable ranges (O’Brien, 2007). 
Missing Data Analysis. AASPIRE took precautions designing the web survey to reduce the problem of missing data. If participants skipped a question, they were notified and given the opportunity to fill it in before continuing the survey. The website required the participant to actively click the response option "Do not know or do not wish to say" in order to continue the survey. Also, SPSS Amos 22.0.0 calculates goodness-of-fit indices without deleting participants or imputing data based on the assumption that data is missing randomly (Arbuckle, 2007). However, some goodnessof-fit indices are impossible to calculate with missing data. Prior to carrying out analyses, I examined the missing data, looking for trends indicating nonrandomness. To test for the randomness of missing data, I constructed a dummy variable containing two groups, cases with missing data and cases without missing data for each of the demographic variables and key variables (Tabachnick \& Fidell, 2007). I conducted a test to determine whether there was a significant difference between the means of these two groups with any scale containing multiple missing data. For the involvement in the online Autistic community variable, multiple missing data were defined as missing over three of the six items of the sense of community scale or any of the single items for years involved, frequency of involvement, or importance of involvement. For the Autistic identity variable, multiple missing data were defined as missing five or more items of the overall Autistic Identity Scale. No participant was missing data for over three items in the self-determination measure; therefore no missing data analyses involved the self-determination measure. No significant differences existed between the groups containing missing data and not containing missing data on the main variables in 
the current study: involvement in the online community, Autistic identity, communication preference, or self-determination. Therefore, missing data was not substantial in the analyses.

Involvement in the online community composite variable. In order to analyze Hypotheses 1 through 4, I created the measurement of involvement in the online Autistic community by determining the weight of each of the four dimensions within the variable. I determined the composite variable for involvement in the online Autistic community with weights assigned according to a principal components analysis. To use a data-driven approach to weight the dimensions of involvement in the online Autistic community, I conducted principal components extraction with an orthogonal rotation. The extraction contained the four dimensions of frequency, years of involvement, sense of community, and importance of involvement. Oblique rotation was appropriate because of the correlations among factors (Tabachnick \& Fidell, 1996). Examining the rescaled components in the component matrix, I used principal components loadings to determine a weight for each dimension. Table 4 contains the individual principal components weights by percentage of each of the four components. Using these weights, I calculated a composite variable containing the weighted dimensions for use in analyses.

Since this approach utilized a data-driven weighting process to compliment the theoretical and community-determined inclusion of each of the four dimensions, the above process was repeated for the composition of involvement in an online community 
for non-autistic individuals. Data-driven weighting differed according to sample; the weighting differed in the current study according to the presence of autism. However, the weights were similarly ranked in the separate samples, with the importance of involvement in the online community with the largest weight (38.3\% in non-autistic individuals and $34.2 \%$ in autistic individuals), frequency of involvement with the second largest weight (28.7\% in non-autistic individuals and $33.3 \%$ in autistic individuals), followed by sense of community $(20.6 \%$ in non-autistic individuals and $24.1 \%$ in autistic individuals), and years of involvement with the smallest weight (12.4\% in non-autistic individuals and $8.4 \%$ in autistic individuals). The determined weights of the dimensions of involvement in an online community for non-autistic individuals were utilized for nonautistic individuals in the analysis of Hypothesis 3 , the only analysis that contains the non-autistic sample.

Examining the factor structure of the Autistic Identity Scale. Prior to conducting hypotheses tests, I tested the factor structure of the Autistic Identity Scale using exploratory factor analysis. During the development of the survey measures, AASPIRE investigators examined the Autistic Identity Scale items to attempt to identify the domains in accordance with Gill's theory to determine if a factor analysis should be exploratory or confirmatory. Through the examination of the scale, the domains did not seem to intuitively converge on theory domains. We then independently assigned items to domains, seeking inter-rater convergence, but were also unable to settle on the item assignment to domains between team members. Due to this lack of alignment between 
the scale items and Gill's theory, an exploratory factor analysis was most appropriate for understanding the unknown factor structure of the Autistic Identity Scale. Confirmatory factor analysis would be appropriate only if we were aware of how items may align with dimensions of the theory.

First, I conducted principal components extraction with an orthogonal rotation, and I examined the Eigenvalues and a Scree test to determine the number of factors (Tabachnick \& Fidell, 1996). There were five factors with Eigenvalues higher than one, which indicated the presence of five factors according to Kaiser's criteria. However, Cattell's Scree Criterion indicated that there were 3 or 4 factors, with no sharp break indicating a clear number of components. In the event of contradicting PCA results, the literature recommends examining a number of solutions prior to deciding the number of factors, and to determine the number of factors based on which solution matches the theoretical grounding of the scale best (Ford, MacCallum, \& Tait, 2006). Therefore, I examined solutions based on the oblique rotation of three, four, and five factors with the 18 items.

Since the different rotations indicated that three factor structure solutions were possible, I determined, examined, and compared each solution for best statistical and theoretical fit with Gill's theory. First, the 18 item scale determined the loadings of the three factor solution. I assigned each item to the factor it loaded highest onto of the three factors according to the pattern and structure matrices. Literature on EFA commonly describes the rule of identifying significant loadings when an item loads on a 
factor greater than .40; however this practice limits viewing patterns of high and low loadings across factors (Fabrigar, Wegener, MacCallum, \& Strahan, 1999; Ford et al., 2006) Therefore, if an item factor loading was over .40 I considered the item for a factor. The range of factor loadings from highest to lowest was also considered for each factor structure solution. The percentage of variance and covariance representing the factors indicated the importance of each factor. Within the factor structure, I examined correlations among subscale means and correlations among individual items within each factor and between factors. Additionally, I calculated Cronbach's alpha for solution's subscales to examine the internal consistency for the scale factor structure. I repeated this process (assigning factors and examining correlations and alphas) in the three factor solution for both the four factor solution and the five factor solution. Once I determined the separate solutions and psychometric properties of subscale and item-level correlations and alpha were determined, I examined the solutions for best statistical and theoretical fit.

Five items were problematic across all factor solutions according to the correlations between items and subscales of each of the solutions as well as alphas of subscales in the solutions. I grouped four of the items ("Overall, people on the autistic spectrum are considered valuable by others," "Most people consider people on the autistic spectrum, on average, to be less effective than people not on autistic spectrum," "In general, others respect people on the autistic spectrum," and "In general, others think that people on the autistic spectrum are unworthy") as a four-item factor in both the three and four factor solutions, and I grouped the first three items 
together as a three-item factor in the five factor solution. In the five factor solution, the item "In general, others think that people on the autistic spectrum are unworthy" loads at .52 on another factor but .48 with the first three items, indicating that it may belong in more than one factor. Upon examining factor correlations, I found that the factor containing all four items was consistently significantly negatively related to other factors in the three solutions. The individual problematic items were significantly positively correlated with one another. However, the items were either not correlated or were significantly negatively correlated with the majority of the other items in the scale. One additional item ("Overall, being a person on the autistic spectrum has very little to do with how I feel about myself") loaded on multiple factors that contained inconsistent items across the solutions, positively correlated with only a third of the items in the scale, and negatively correlated with two other items in the scale. This item was significantly and strongly correlated with a similar item ("Being a person on the autistic spectrum is not important to my sense of what kind of person I am"), suggesting that the inclusion of a poorly performing item was unnecessary. In all, the alpha of the overall scale rose from .76 to .84 upon removing the five items. Additionally, the poorly performing items in the EFA were some of the items that research team members identified as difficult to organize into Gill's disability identity theory dimensions when attempting to discern separate subscales that match the dimensions. As no measurement development had been conducted on this scale in previous research, there was a possibility that adjustments needed to be made to improve the scale (Tabachnick \& Fidell, 1996). Therefore, I revised the scale to include 13 of the original 18 
items. None of the remaining items were problematic in terms of item-level correlations or alphas. That is, the item-level correlations were not negative across the scale items and the alphas were acceptable.

I performed another PCA containing 13 of the 18 items after I removed the five poorly performing items. The eigenvalues then indicated a three-factor model, while the scree plot again indicated an unclear number of factors. Since EFA is an exploratory technique which may be interpreted in a number of ways, I performed the EFA utilizing a number of different procedures to determine the number of factors (Thompson, 2004), including trying various numbers of solutions and types of rotations. This exploratory approach allowed me to come to a final solution that aligned most both conceptually and statistically with the factor model.

First, I narrowed the rotation method to only oblique rotation methods since the majority of the individual items are significantly correlated with one another. Next, as the theory of disability identity was applied in writing the items, I attempted to interpret the factor loadings of the three, four, and five factor solutions of the 13-item scale by Gill's theory of disability identity (1997a), assigning a theoretical domain to each of the factors. I determined the solution with oblique rotation of four forced factors in exploratory factor analysis created the most theoretically sound arrangement of items in factors (Thompson, 2004). That is, after examining the loadings onto the three, four, and five factor solutions, only the four factor solution contained items under each factor that conceptually resonated with a framework that may be interpreted via the disability and Autistic identity literature. Although the eigenvalue of the fourth component was 
under 1.0, the eigenvalue was .99, or just .01 under Kaiser's Criterion. The four factor solution now represented each of the four dimensions of the Autistic identity theory adapted from Gill's (1997a) disability identity theory: (1) Integration into society, (2) Integration into the Autistic community, (3) Internal integration of sameness and differentness with society and the Autistic community, and (4) Integration of feelings about self and presentation of self. The selected items corresponded to the proposed structure of Gill's four dimensions.

After I determined that the four factor solution will be utilized, I examined factor loadings of the pattern and structure matrices for strength of relationship between each item and its respective factor (Tabachnick \& Fidell, 1996). Any items with factor loadings lower than .40 indicate poorly performing items. No remaining items of the 13 -item scale load less than .40 on at least one factor. However, six of the items loaded higher than .40 on more than one factor. Factor loadings should be determined not only by factor loadings over .40 but also theoretical alignment with factors, internal consistency reliability, and examination of overall loadings on each factor (Fabrigar et al., 1999; Ford et al., 2006). Therefore, I examined each of these items for theoretical basis of inclusion in the factor on which each loaded higher, or whether any items should be theoretically included in more than one factor.

The factor named "Integration of feelings about self and presentation of self" contained two items that loaded to a lesser degree onto other factors. The item "Being a person on the autistic spectrum is an important reflection of who I am" loaded onto the factor at .67, and also loaded in the factor named "Internal integration of sameness 
and differentness with society and Autistic community" at .60. However, the item is less conceptually aligned with this factor since this factor focuses more on resolving feelings about differences between autistic individuals and non-autistic individuals rather than how the item is specifically about internal reflection of how close an Autistic identity is to an individual. The item "Being a person on the autistic spectrum is not important to my sense of what kind of person I am" loaded onto the factor at .67, and also loaded on the factor named "Integration into society" at .47, but is less conceptually aligned with this factor as well, which focuses less on the closeness of an Autistic identity to the individual and more on feels of belonging with society in general. Four additional items conceptually fit with only the factor named "Integration into the Autistic community" since they both directly relate to feelings about others in the Autistic community more than feelings about self. The item "I feel that people on the autistic spectrum have made major accomplishments and advancements" loaded onto "Integration into the Autistic community" (.46), "Integration into society" (.42), and "Integration of society and the Autistic community" (.54). The item "My future is tied to the future of other people on the autistic spectrum" loaded onto "Integration into the Autistic community" (.48) and "Integration of feelings about self/presentation of self" (.68). The item "I have a strong sense of belonging to the Autistic community" loaded onto "Integration into the Autistic community" at .66, onto "Integration of society and the Autistic community" at .41, and onto "Integration of feelings about self and presentation of self" at .57. Lastly, "I almost never tell people I am on the autistic spectrum" loaded onto "Integration into the Autistic community" (.59) and "Integration of society and the Autistic community" (.44). 
I checked the internal consistency of each of the subscales with the possible item arrangements in the different factors possible; the above arrangement of items that loaded onto more than one factor resulted in the highest subscale alphas in addition to the best conceptual fit.

Reflecting the findings of the EFA, the revised Autistic Identity Scale that was used for all further analyses in this study is compromised of 13 items in four subscales. The overall scale showed good internal consistency reliability (Cronbach, 1951) in the current sample $(\alpha=.84)$. The overall alpha level indicated that despite multidimensionality, the subscales and individual items each contribute to measuring the overall construct of Autistic identity. The subscale "integration into society" contains two items that measure a person's rights and belief in belonging to society, or feeling as though autistic individuals deserve inclusion and acceptance. This subscale showed acceptable reliability (Cronbach, 1951) in the current sample $(\alpha=.71)$. The subscale "integration into the Autistic community" contains five items that measure a person overcoming negative feelings about being autistic to find rewarding relationships with other autistic individuals, which are important to the individual. This second subscale also showed acceptable reliability in the current sample $(\alpha=.70)$. The third subscale, "internal integration of similarities and differences with others," contains three items that measure a person's resolution of the conflict of self as broken or not whole with a sense of pride and belonging with identifying as autistic. The third subscale showed acceptable reliability in the current sample $(\alpha=.78)$. Lastly, "integration of internal feelings of self with presentation of self" contains three items that measure how 
comfortable individuals are with themselves, characterized by a lack of internal conflict or discomfort with being autistic, and seeing autism as an essential part of self. The fourth subscale showed acceptable reliability in the current sample $(\alpha=.77)$. While each of the subscales had acceptable reliability, higher alpha levels would indicate good or excellent reliability. I took into consideration the somewhat low alpha levels for the analyses for the current study by not conducting hypothesis testing with subscales, since the overall alpha of .84 is considered good. Table 5 displays the results of the EFA, including the four factors of the determined solution and item factor loadings.

Inter-construct correlations. I calculated the relationships between constructs with Pearson's correlation coefficient to ensure that variables were not measuring the same underlying construct in both the autistic and non-autistic samples. Table 6 displays the correlation matrix. Overall, correlational analyses suggested significant moderately positive relationships among the constructs of involvement in the online community, self-determination, and Autistic identity. Correlational analyses showed no highly correlated (e.g., $r=.80$ or higher) relationship, which could have presented issues of multicollinearity (Tabachnick \& Fidell, 1996). I found no significant relationships with communication preference and involvement in the online community, Autistic identity, or self-determination in either sample.

\section{Hypotheses Tests.}

The analyses tested one overarching aim and four specific research questions. I first examined the relationship between involvement in the online Autistic community 
and Autistic identity $(\mathrm{H}$ 1.1), and whether communication preference moderates the relationship ( $H$ 1.2). I then tested the relationship between involvement in the online Autistic community and self-determination $(\mathrm{H} 2.1)$, and whether communication preference moderates the relationship (H 2.2). Next, I tested whether communication preference has a stronger moderating effect on the relationship between involvement in the online community and self-determination in autistic adults and non-autistic adults (H 3). Finally, I tested that the Autistic Identity Scale is a plausible representation of the dimensions of Autistic identity ( $\mathrm{H}$ 4.1), and that positive relationships among involvement in the online Autistic community, Autistic identity, and self-determination is a plausible representation of the structural model $(\mathrm{H} 4.2-4.7)$, with relationships stronger in autistic adults who prefer online communication than in autistic adults who do not prefer online communication (H 4.8). I describe each hypothesis test in this section. Significance levels for all analyses were set at $p<.05$.

\section{Hypothesis 1.1. The importance of involvement in the online Autistic} community will be positively related to Autistic identity. To evaluate hypothesis $1.1, \mathrm{I}$ computed the correlation between involvement in the online Autistic community and Autistic identity in autistic adults. I only included participants in the analysis if they had a composite score for involvement in the online Autistic community. That is, the analysis only included participants if they responded to the items on years involved, frequency of involvement, importance of involvement, and at least $50 \%$ ( 3 out of 6 items) of each SCl-2 subscale included in the composite score $(n=113$, total autistic sample $n=151$; 
$74.8 \%)$. The minimum requirement of 3 out of 6 items for each $\mathrm{SCl}-2$ subscale was to allow the maximum number of participants to be included in the analyses without sacrificing the integrity of the mean scores. The correlational analysis supported the hypothesis that involvement in the online Autistic community is positively related to Autistic identity $(n=113, r=.33, p<.01)$ in autistic adults ${ }^{1}$. I performed a post-hoc power analysis, and determined that the power was .95. The correlation between involvement in the online Autistic community and Autistic Identity is a medium-strength positive relationship (Cohen, 1988), and the high power coefficient indicates a high amount of power for rejecting the null hypothesis.

I conducted post-hoc analyses to further inform the relationship between involvement in the online Autistic community and Autistic identity in autistic adults by examining correlations between the dimensions of involvement in the online Autistic community and the subscales of Autistic identity. The correlations between the overarching constructs and their respective dimensions can be found in 0 . In summary, the construct of involvement in the online Autistic community is significantly positively related to the two Autistic identity dimensions of integration of sameness and differentness in society and the Autistic community $(r=.26, p<.01)$ and integration into the Autistic community $(r=.54, p<.01)$, but not significantly related to the dimensions of integration into society or integration of feelings of self and presentation of self. The overall Autistic identity construct is significantly positively correlated to three of the

\footnotetext{
${ }^{1}$ An analysis of Hypothesis 1.1 with the sample of only autistic adults with an AQ score 26 or higher also yielded significant results: $n=100, r=.44, p<.01$. With only adults with an AQ score of 32 or higher, $\mathrm{n}=89, \mathrm{r}=.46, \mathrm{p}<.01$.
} 
dimensions of involvement in the online Autistic community, including sense of community $(r=.21, p<.05)$, importance of involvement $(r=.34, p<.01)$, and frequency of involvement $(r=.27, p<.01)$. Autistic identity is not significantly related to years of involvement.

Hypothesis 1.2. Communication preference will moderate the relationship between involvement in the online Autistic community and Autistic identity, with a more positive relationship between involvement in the online Autistic community and Autistic identity when a person prefers online communication over when a person does not prefer online communication. In order to test hypothesis 1.2 , I conducted a moderated regression with the variables of involvement in the online Autistic community, communication preference, and Autistic identity in autistic adults (see Figure 2). I centered the continuous variable (Aiken \& West, 1991) of involvement in the online Autistic community to provide a meaningful zero point for the slope and intercepts of the regression output by subtracting the mean from each case's score. I utilized dummy coding for the categorical moderator variable. Since the categorical moderator contains two comparison groups rather than a baseline group and comparison group, I coded "does not prefer online communication" and "prefers online communication," respectively as "-1" and "1" instead of including a zero group as the baseline (Aiken \& West, 1991). This provided the desired contrast between the two communication preference groups. I then created an interaction term (Aiken \& West, 
1991) for involvement in the online Autistic community and communication preference by computing a variable of the product of the two variables.

I entered Autistic identity as the dependent variable of the regression model, and entered three independent variables: centered involvement in the online Autistic community, communication preference, and the interaction of centered involvement in the online Autistic community and communication preference. The overall model was statistically significant, $F(3,106)=5.49, p<.01, f^{2}=.16$ and accounted for approximately $11 \%$ of the variance of Autistic identity $\left(R^{2}=.14\right.$, Adjusted $\left.R^{2}=.11\right)$, a low to medium amount of variance. A post hoc power analysis revealed a power statistic of .94, indicating very strong power to reject the null hypothesis. Despite overall significance of the model, the only significant individual predictor was the variable of involvement in the online Autistic community, $\beta=.32, t(109)=3.49, p<.01,95 \% \mathrm{Cl}=.119, .433$. Communication preference $[\beta=-.14, t(109)=-1.57, p=.12,95 \% \mathrm{Cl}=-.266, .031]$ and the interaction of communication preference and involvement in the online Autistic community $[\beta=.11, t(109)=1.25, p=.21,95 \% \mathrm{Cl}=-.058, .156]$ were not significant in the model $^{2}$. Therefore, the results of the moderated regression did not support the hypothesis that communication preference moderates the relationship between involvement in the online Autistic community and Autistic identity in autistic adults. Table 8 displays the results of the moderated regression.

${ }^{2}$ An analysis of Hypothesis 1.2 with the subset of autistic adults with $A Q$ cut-off scores 26 or higher yielded a statistically significant overall model, $F(3,95)=9.00, p<.01, R^{2}=.22$, Adjusted $\mathrm{R}^{2}=.20$. No predictors were significant with this subset of the sample. In autistic adults with an $A Q$ cut-off score of 32 or higher, the overall model was statistically significant $F(3,84)=9.69, p<.01$, $R^{2}=.26$, Adjusted $R^{2}=.23$. No predictors were significant in the sample with an $A Q$ cut-off of 32 . 
I examined correlations in autistic adults among the constructs Autistic identity, involvement in the online Autistic community, and communication preference, as well as respective dimensions. In examining the correlations, communication preference was only significantly correlated with one dimension of the involvement in the online Autistic community - frequency of involvement $(r=.19, p=.03)$. Since communication preference is not correlated with the overall constructs and the majority of the dimensions of the constructs, I conducted no further analyses to test the hypothesis involving involvement in the online Autistic community, Autistic identity, and communication preference.

\section{Hypothesis 2.1. Involvement in the online Autistic community will be positively}

related to self-determination. To evaluate hypothesis 2.1 , I computed the correlation between involvement in the online Autistic community and self-determination in autistic adults. I only included participants in the analysis if they had a composite score for involvement in the online Autistic community $(n=113)$. No participant was missing data for over three items in the self-determination measure; therefore I excluded no participants for missing data in the self-determination measure $(n=151)$. The result of the correlation analysis supported the hypothesis that involvement in the online Autistic community is positively related to self-determination in autistic adults ( $n=113, r=.38$, $p<.01)^{3}$. I performed a post-hoc power analysis, and determined that the power was.99.

The correlation between involvement in the online Autistic community and self-

\footnotetext{
${ }^{3}$ An analysis of Hypothesis 2.1 with the sample of only autistic adults with an AQ score 26 or higher also yielded significant results: $n=103, r=.39, p<.01$. With only adults with an AQ score of 32 or higher, $n=91, r=.36, p<.01$.
} 
determination is a medium-strength positive relationship (Cohen, 1988), and the high power coefficient indicates a high amount of power for rejecting the null hypothesis.

I conducted post-hoc analyses to further inform the relationship between involvement in the online Autistic community and self-determination by examining correlations between the dimensions of involvement in the online Autistic community and subscales of self-determination in autistic adults. The correlations between the overarching constructs and their respective dimensions can be found in Table 10. In summary, self-determination is significantly positively related to sense of community $(r=.56, p<.01)$ and importance of involvement $(r=.32, p<.01)$, but not significantly related to years of involvement or frequency of involvement. Involvement in the online Autistic community is only significantly related to one of the three dimensions of selfdetermination, relatedness, with which it has a strong positive relationship $(r=.68$, $p<.01)$; involvement in the online Autistic community is not related to autonomy or competence.

Hypothesis 2.2. Communication preference will moderate the relationship between involvement in the online Autistic community and self-determination, with a more positive relationship between involvement in the online Autistic community and self-determination when a person prefers online communication over when a person does not prefer online communication. I conducted a moderated regression with the variables of involvement in the online Autistic community variable, communication preference, and self-determination (see Figure 3). As in Hypothesis 1, I utilized the 
centered variable (Aiken \& West, 1991) of involvement in the online Autistic community to provide a meaningful zero point for the slope and intercepts of the regression output. I utilized dummy coding for the categorical moderator variable, with "does not prefer online communication" coded as "-1" and "prefers online communication" coded as " 1 " instead of using zero as a dummy code to indicate a baseline group. This provided the desired contrast between the two communication preference groups. I also utilized the interaction term for involvement in the online Autistic community and communication preference.

I entered self-determination as the dependent variable of the regression model, and entered three independent variables: centered involvement in the online Autistic community, communication preference, and the interaction of centered involvement in the online Autistic community and communication preference. The overall model was statistically significant, $F(3,106)=6.152, p<.01, f^{2}=.19$ and accounted for approximately $12 \%$ of the variance of self-determination $\left(R^{2}=.15\right.$, Adjusted $\left.R^{2}=.12\right)$. A post hoc power analysis revealed a power statistic of .97 , indicating very strong power to reject the null hypothesis. Despite overall significance of the model, the only significant predictor was the variable of involvement in the online Autistic community, $\beta=.30, t(109)=2.20, p<.01$, $95 \% \mathrm{Cl}=.011, .216$. Communication preference $[\beta=-.01, t(109)=.-.06, p=.95,95 \% \mathrm{Cl}=-$ $.133, .126]$ and the interaction of communication preference and involvement in the online Autistic community $[\beta=.10, t(109)=.76, p=.45,95 \% \mathrm{Cl}=-.084, .188]$ were not 
significant in the model ${ }^{4}$. Therefore, the results of the moderated regression did not support the hypothesis that communication preference moderates the relationship between involvement in the online Autistic community and self-determination. Table 111 displays the results of the moderated regression.

I examined correlations among the constructs self-determination, involvement in the online Autistic community, and communication preference, as well as respective dimensions. As in the moderated regression model containing Autistic identity, only one significant correlation existed between communication preference and frequency of involvement. Since communication preference is not correlated with the overall constructs and the majority of the dimensions of the constructs, I conducted no further analyses for the hypothesis including involvement in the online Autistic community, selfdetermination, and communication preference.

Hypothesis 3. Communication preference will have a stronger moderating effect on the relationship between involvement in the online community and selfdetermination in autistic adults than non-autistic adults. To evaluate hypothesis 3,1 tested the multiple moderation of communication preference and population (autistic or non-autistic) on the relationship between involvement in the online community and self-determination. I conducted a multiple moderated regression with the variables of

\footnotetext{
${ }^{4}$ An analysis of Hypothesis 2.2 with the subset of autistic adults with AQ cut-off scores 26 or higher yielded a statistically significant overall model, $F(3,96)=6.0 ., p<.01, R^{2}=.16$, Adjusted $R^{2}=.13$. No predictors were significant with this subset of the sample, but the variable of involvement in the online Autistic community was nearly significant, $\beta=.28, t(100)=1.90, p=.06$. In autistic adults with an $A Q$ cut-off score of 32 or higher, the overall model was statistically significant $F(3,85)=4.73$., $p<.01, R^{2}=.14$, Adjusted $R^{2}=.11$. No predictors were significant in the sample with an AQ cut-off of 32 .
} 
involvement in the online community variable, communication preference, population type (autistic or non-autistic), and self-determination (see Figure 4).

For the analysis of hypothesis 3, I utilized a higher-order interaction, in which a three-way interaction was interpreted to determine differences between autistic and non-autistic groups on the moderation of communication preference. The standards for centering coding, hypothesis testing, and effect size generalize to higher-order interactions (Aiken \& West, 1991), so the analysis of hypothesis 3 was similar to the analysis of hypothesis 2.2 with the addition of the population type (autistic or nonautistic) categorical variable and the interpretation of a three-way interaction. Further, I utilized the composite variable of involvement in an online community for non-autistic individuals for the non-autistic group in this analysis. I also utilized the composite variable of involvement in the online Autistic community for the autistic group in this analysis.

I created a centered variable of involvement in the online community to provide a meaningful zero point for the slope and intercepts of the regression output. I utilized dummy coding for the communication preference moderator variable as in prior analyses. Unlike the autistic sample, a chi-square test indicated significant differences on the communication preference variable in non-autistic individuals $\left(\chi^{2}=21.09, p<.01\right)$, making the overall sample compromised of approximately $60 \%$ individuals who do not prefer online communication. The literature recommends weighted effect coding in the case of unequal frequencies in the two levels of the categorical moderator (Aguinis, 
2004; Aiken \& West, 1991). Therefore, I dummy coded the communication preference variable for the analysis as .40 for individuals who do not prefer online communication and -.60 for individuals who prefer online communication. I also utilized dummy coding for the population variable (Aiken \& West, 1991), with autistic individuals coded as "1" and non-autistic individuals coded as "-1." These dummy codes provided the desired contrast between the two communication preference groups and the autistic or nonautistic groups.

I performed a multiple regression with self-determination as the dependent variable with six independent variables: involvement in the online community, communication preference, population type, interaction of involvement and communication preference, interaction of involvement and population type, and threeway interaction of involvement, communication preference, and population type. The overall model was statistically significant, $F(6,240)=11.71, p<.05, f^{2}=.33$ and accounted for approximately $21 \%$ of the variance of self-determination $\left(R^{2}=.23\right.$, Adjusted $\left.R^{2}=.21\right)$. A post hoc power analysis revealed a power statistic of .99 , indicating very strong power to reject the null hypothesis. Despite overall significance of the model, the predictors were not all significant. Similarly to the regression of self-determination on involvement in the online Autistic community $(\mathrm{H} 2.2)$, the regression of self-determination on involvement in the online community was significant in the current regression model, $\beta=$ $.39, t(246)=6.39, p<.05,95 \% \mathrm{Cl}=.110, .208$. Communication preference was also significant in this model, $\beta=.16, t(246)=2.54, p<.01,95 \% \mathrm{Cl}=.027, .211$. The last significant predictor in this model was population type, $\beta=-.20, t(246)=-3.26, p<.01,95 \%$ 
$\mathrm{Cl}=-.121,-.030$. None of the interaction variables were significant, including the interaction of involvement and communication preference $[\beta=-.07, t(246)=-1.2, p=.23$, $95 \% \mathrm{Cl}=--.160, .038]$, the interaction of involvement and population $[\beta=-.04, t(246)=-$ $.60, p=.55,95 \% \mathrm{Cl}=-.063, .034]$, or the three-way interaction of involvement, communication preference, and population $[\beta=.03, t(246)=.41, p=.68,95 \% \mathrm{Cl}=-.079$, .121]. Therefore, the results of the moderated regression did not support the hypothesis that the moderation of communication preference on relationship between involvement in the online community and self-determination is dependent on whether a person identifies as autistic.

I conducted post hoc analyses to further understand the findings of the threeway moderated regression. I found that self-determination was higher in non-autistic individuals $(M=2.83, S D=.38)$ than in autistic individuals $(M=2.64, S D=.38), t(322)=-4.88$, $p<.01$. Further, self-determination was significantly higher in individuals who do not prefer online communication $(M=2.79, S D=.39)$ than individuals who do prefer online communication $(2.70, S D=.40)$ in the full sample, $t(336)=2.30, p<.05$. No significant differences existed in involvement in the online community between non-autistic and autistic individuals. However, all individuals who prefer online communication $(M=4.41$, $S D=.91$ ) had significantly higher involvement in the online community than individuals who do not prefer online communication $(M=4.16, S D=0.92), t(245)=-2.09, p=.04$. Despite these significant differences on the two variable level, the interactions of variables were not significant. I conducted further post-hoc analyses within the autistic adult sample. Between autistic adults who prefer online communication and autistic 
adults who do not prefer online communication, there were no significant differences on the variables of involvement in the online Autistic community $[t(108)=-1.37, p=.17]$ or self-determination $[t(143)=.438, p=.66]$.

Hypothesis 4. The conceptual model is a plausible representation of the relationships among constructs, including the Autistic Identity Scale representing the dimensions of Autistic identity (4.1) and the positive relationships among involvement in the online Autistic community, Autistic identity, and self-determination (4.2-4.7), and that the relationships in the model will be stronger in autistic adults who prefer online communication than in autistic adults who do not prefer online communication (4.8). To evaluate hypotheses 4 , I used SPSS AMOS 21.0 to test the structure of the proposed model containing involvement in the online Autistic community, Autistic identity (compromised of the dimensions of the Autistic identity scale as determined by the preliminary EFA of the scale), and self-determination in two groups (prefer online communication, does not prefer online communication). Here I discuss the process of building the model, modifying the model, replicating the model in the two subscales for comparisons, and the process for comparing fit in the two models. Then I discuss specific analysis findings as they related to Hypotheses 4.1 through 4.8 .

I first modeled the relationships among the variables of interest in the sample of autistic adults. The structural model included the weighted involvement in the online Autistic community variable as an observed exogenous variable, or a measured variable with no variables within the model predicting or causing involvement in the online 
Autistic community. The model also contains Autistic identity as an unobserved exogenous variable (latent variable with no causes included in the model), the Autistic Identity Scale dimensions as four observed endogenous variables (measured variables that represent dimensions of Autistic identity variable), and a measurement error term on each of the dimensions as four unobserved exogenous variables (latent disturbances). Lastly, the model contained the mean for self-determination as an observed exogenous variable (latent variable with no causes included in the model). The latent variable of Autistic identity and the two observed variables of involvement in the online Autistic identity and self-determination were each set to co-vary with one another, since the hypothesized model indicated that each of the variables have bidirectionally relationships. Overall, the recursive model contained 24 parameters, including 5 fixed parameters and 19 distinct free parameters to be estimated.

The recommended sample size for SEM varies in the literature. Authors recommend using a minimum of 200 cases with smaller sample sizes acceptable for simpler model that contain no or few latent variables (Kenny, 2012). Alternatively, others recommend using a ratio of sample size to the number of free parameters, with a goal ratio of 5 to 1 (Bentler \& Chou, 1987). However, many published studies do not meet this ratio goal. With the variety of recommendations, recommended minimum sample sizes or minimum ratios tend to be thought of as suggestions rather than necessary requirements (Kenny, 2012). In the overall model that the present study analysis uses, the sample size was 151 , which may be sufficient for the analysis if following the rule of thumb for 5 to 1 ratio ( $n=95)$. However, AMOS uses listwise 
deletion and performs no alternative types of deletion or estimation for any cases missing data, and there were 29 cases containing missing data on one or more of the dimensions of Autistic identity or Involvement in the online Autistic community. Further, the sample recommendation would not be sufficient in the comparison model that would have 74 autistic adults who prefer online communication and 71 autistic adults who do not prefer online communication. In order to maximize the accuracy of estimations in the model with the largest sample size possible, the analyses should not utilize listwise deletion as in prior analyses. Instead, I conducted mean imputation (Carter, 2006) of individual dimensions of each variable containing missing data (involvement in the online Autistic community and Autistic identity). Mean imputation involves substituting missing cases with the overall sample mean for each variable. This method of imputation is recommended over listwise deletion for SEM, despite the drawback that mean imputation does not take into consideration participant-level patterns of scores across other variables (Carter, 2006). Other types of imputation, such as regression imputation or pattern matching may be more accurate in replacing missing data due to less bias in variance, but mean imputation retains the overall means of variables (Kline, 1998), which were used for all other analyses in the current study, creating uniformity across analyses that allows for comparison of findings. Additionally, only $16.56 \%$ of the cases required imputation for the analysis. Four cases required mean imputation of one of the dimensions of the Autistic identity dimensions, and 25 cases required mean imputation of involvement in the online Autistic community dimension 
scores. Then I calculated the weighted mean scores for involvement in the online Autistic community with complete data including mean imputation.

After I addressed missing data, I ran the structural model with the sample containing all autistic adults. I examined the absolute fit indices to determine how well the hypothesized model fits the sample data (McDonald \& Ho, 2002). Absolute fit indices provided information on how well the hypothesized model fit the data in comparison to no model (Jöreskog \& Sörbom, 1993). See Table 13 for all fit indices. Of the absolute indices, the literature strongly recommends the consideration of the chisquare, RMSEA, and SRMR (Boomsma, 2000; Hooper, Coughlan, Mullen, 2007; Kline, 2005). The chi-square was significant, $\chi^{2}(\mathrm{df}=8)=22.85, p<.01$, indicating poor model fit (Barrett, 2007). However, the literature often discusses chi-square significance in SEM as an inadequate measure of model fit that is too liberal (i.e., too many Type 1 errors; Kenny, 2012). The RMEA was .11 for the model (.26 in the independence model), indicating poor model fit (Hooper, Coughlan, \& Mullen, 2008; MacCallum et al., 1996). The $p$ of Close Fit (PCLOSE) is a measure of the null hypothesis that the RMSEA is significant as a close-fitting model if greater than .05 (Kenny, 2012). The model had a PCLOSE of .03, again indicating a poorly fitting model. The Standardized Root Mean Square Residual (SRMR) is an absolute measure of fit defined as the standardized difference between the observed correlation and the predicted correlation, with a value less than .08 indicating good model fit. The SRMR of the model was .06 , indicating good model fit (Hooper, Coughlan, \& Mullen, 2008). The incremental fit indices measure the null hypothesis that all variables are uncorrelated (McDonald \& Ho, 2002). The literature 
recommends the consideration of at least the Comparative Fit Index (CFI) for incremental fit indices (Boomsma, 2000; Hooper, Coughlan, \& Mullen, 2007; Kline, 2005). The CFI was .90, which is the cut-off for a good-fitting model (Hu \& Bentler, 1999).The Normed Fit Index (NFI) was .86-below the .90 cut-off for a good-fitting model (Bentler \& Bonnett, 1980). The overall model therefore offered a relatively weak fit.

Since the fit of the overall model was relatively weak with fit indices offering mostly poor fitted indices of model fit, I examined modification indices to identify any improvements to the model to improve its fit with the Lagrange Multiplier test (Kline, 1998). The covariance of the error terms of two of the Autistic identity dimensions (integration into society and internal integration of sameness and differentness of society and the Autistic community) had a modification index of 6.76 with an expected parameter change of .21 . By adding a free parameter of a covariance between the two error terms, the estimate becomes .21 larger than the original model. That is, the modification makes the model a better fit. Hooper, Coughlan, and Mullen (2007) recommend that rather than adding a covariance term, terms with a regression weight less than .20 should be removed from the model rather than adding a covariance to account for error. However, the two dimensions had standardized regression weights over .20. Correlating error terms is not considered an ideal practice when correlating terms from different variables (Gerbing \& Anderson, 1984). However, within-variable correlated terms is justifiable given theoretical justification of such a correlation (Jöreskog \& Long, 1993). The model contains relationships and variables that have not 
been previously studied, and the measure of Autistic identity with four respective dimensions was determined only by the EFA conducted prior to this SEM analysis. Therefore, I accepted this change to the model, correlating the two error terms, based on the modification indices for improvement in model fit and the minimal impact on the overall theoretical and statistical model.

I re-ran the model with the added free parameter of a covariance between the error terms of two of the Autistic identity dimensions. See Figure 7 for the complete model imputed into AMOS for the model, with 20 free and distinct parameters estimated. The complete model was recursive with non-correlated error terms and no feedback loops between endogenous variables (only bidirectional relationships among variables), which creates a more stable and straightforward model (Kline, 1998). Despite the significance of the chi-square indicating poor model fit $\left[\chi^{2}(d f=7)=14.50, p=.04\right]$, other absolute fit indices showed a good model fit (RMSEA=.08, PCLOSE=.16, SRMR=.05). Incremental fit indices also indicated good model fit (NFI=.91, $\mathrm{CFI}=.95)$. Additionally, the added covariance between the error terms of the Autistic Identity Scale dimensions of Integration of sameness and differentness with society and Autistic community and Integration with society was significant, $\operatorname{cov}=.25, \mathrm{SE}=.09, \mathrm{CR}=2.75, \mathrm{p}<.01, \mathrm{r}=.28$, confirming the importance of including the relationship present in the model. Since the modified model fit the data containing all autistic adults, I utilized the structure of the model for the comparison of autistic adults who prefer online communication and autistic adults who do not prefer online communication. Further, I utilized this model to 
assess the plausibility of the Autistic identity theory with the Autistic Identity Scale in Hypothesis $4.1^{5}$.

Once I determined that the structure of the model was a good fit, I replicated the model and analyzed in the group of autistic adults who prefer online communication in order to support or reject Hypotheses 4.2 through 4.4 regarding the relationships among variables in the model. As in the model containing the whole sample of autistic adults, the model estimated 20 parameters containing involvement in the online Autistic community, Autistic identity, the four dimensions of Autistic Identity, the four measurement error terms on each of the dimensions, and self-determination. The three variables of interest were set to co-vary with one another, as well as the error terms of two of the Autistic identity dimensions. The sample of autistic adults who prefer online communication was 74 .

The absolute fit indices showed a poor model fit with a significant chi-square $\left[\chi^{2}(d f=7)=14.67, p=.04\right]$ and a RMSEA value over .10 (MacCallum et al., 1996) with RMSEA=.12. However, the other absolute fit indices indicated good model fit, including the PCLOSE of .09 (over .05 cut-off) and the SRMR of .06 (under the .08 cut-off). Similarly, the incremental fit index CFI indicated good model fit of .93. The modification indices recommended improving the model fit by adding another covariation of error term associated with one of the Autistic identity dimensions (Integration of sameness and differentness with society and the Autistic community) with self-determination.

\footnotetext{
${ }^{5}$ I tested the final modified model for fit in the subsample of autistic adults with AQ scores of 26 or higher. The chi-square indicated poor model fit as in the larger sample, $\chi^{2}(\mathrm{df}=8)=22.85$, $\mathrm{p}=.004$. Other fit indices were RMSEA $=.11, \mathrm{PCLOSE}=.029, \mathrm{NFI}=.86, \mathrm{CFI}=.90$, indicating poor model fit likely due to the analysis requiring a larger sample.
} 
While the factor structure of the Autistic Identity Scale has only been examined in the current study, adding this covariance would be based purely on the statistical modeling rather than theoretical grounds. Since the literature does not prefer the practice of correlating error terms in SEM (Gerbing \& Anderson, 1984), the model should not have an additional set of correlated error terms. Further, the change would make only a small statistical difference $(\mathrm{Ml}=4.92$, expected parameter change $=.08)$, and the mixed results of fit indices may be a result of small sample size (Hooper, Coughlan, \& Mullen, 2008). Since the model was a good fit in the overall sample and a marginally good fit in the autistic adults who prefer online communication, I utilized the model for hypothesis testing and as the configural mode for comparison with autistic adults who do not prefer online communication.

I next used the configural model as a basis for comparison to test for a model of complete invariance where the overall model including all parameters fits the data of both the configural model and the comparison model (Kenny, 2011), which are the two groups of autistic adults who do and do not prefer online communication. The comparison also indicated invariance of covariance where the covariance of the model fits the data of both groups, and invariance of factor loadings, where factor loadings are the same across groups (Kenny, 2011). I ran a multiple group comparison in AMOS with the group that prefers online communication $(n=74)$ as the configural group (originally modeled group) and the group that does not prefer online communication $(n=71)$ as the comparison group. The comparison for the two group analysis compared the configural model (parameters were free) with a comparison model in which the covariances 
among involvement in the online Autistic community, Autistic identity, and selfdetermination were set to be equal in the two models, as well as the loadings of the Autistic identity dimensions. The comparison model contained 20 free parameters to be estimated. Model fit would indicate that the same structural model tested in the overall sample and the sample of only autistic adults who prefer online communication similarly fits the comparison model of autistic adults who do not prefer online communication. In the two-group comparison, the chi-square difference between the two models for autistic adults who do and do not prefer online communication was nonsignificant, with $\chi^{2}(d f=6)=6.98, p=.32$. The differences between the model fit indices included $\mathrm{NFI}(\Delta-1)=.04, \operatorname{IFI}(\Delta-2)=.04, \operatorname{RFI}(\mathrm{P}-1)=-.03, \operatorname{TLI}(\mathrm{P}-2)=-.03$. The fit indices across two models indicate measurement invariance across the two groups. Measurement invariance indicates that the parameters within the two models fit the comparison model equally as well as the configural model. Therefore, the fit indices support the null hypothesis that the proposed model (including covariance among involvement in the online Autistic community, self-determination, and Autistic identity and loadings of the four dimensions of Autistic identity) is the same for all autistic adults, regardless of whether or not they prefer online communication.

Lastly, since there was no significant difference between model fit with the configural and comparison models, I considered the structural model a good model fit and utilized the model for the comparison. Absolute indices support that the structural model was a good fit for autistic adults who do not prefer online communication, with a 
nonsignificant chi-square $\left[\chi^{2}(d f=7)=6.57, p=.94\right]$, a RMSEA less than .01, a PCLOSE of .59, and an SRMR of .05. The incremental fit index CFI of 1.00 also indicated good fit.

In summary, I identified and tested the proposed structural model in the overall sample of autistic adults. I made one modification to the model in order to improve model fit, which was to correlate the error terms on two of the Autistic identity dimensions. I tested the structural model in autistic adults who prefer online communication, and found the model to fit marginally well. By constraining free parameters, the autistic adults in the two groups that prefer and do not prefer online communication were found to equally fit the structural model. Lastly, I tested the model in autistic adults who do not prefer online communication to confirm model fit. Next, I discuss how the models generated and tested support or reject Hypotheses 4.1 through

\section{8.}

\section{Hypothesis 4.1. The Autistic Identity Scale is a plausible representation of Autistic} identity as adapted from Gill (1997a). I examined the overall model containing all autistic adults to determine the appropriate representation of the latent Autistic identity variable with the subscales previously determined by the EFA. Specifically, I examined the regression weights of the overall model to determine good fit. I fixed one of the regression paths to properly identify the model. The fixed path which pointed to the subscale Integration of sameness and differentness with society and Autistic community had a standardized regression weight of .73 . The remaining three paths were significant, including the path to the subscale Integration into society 
(unstandardized estimate $=.40, \mathrm{SE}=.13, \mathrm{CR}=3.18, \mathrm{p}<.01$; standardized regression weight=.30), the path to the subscale Integration of feelings of self and presentation of self (unstandardized estimate $=.79, \mathrm{SE}=.15, \mathrm{CR}=5.39, \mathrm{p}<.01$; standardized regression weight $=.56$ ) and the path to the subscale Integration into the Autistic community (unstandardized estimate $=.96, \mathrm{SE}=.17, \mathrm{CR}=5.65, \mathrm{p}<.01$; standardized regression weight=.75).

The covariance between two error terms of the Autistic Identity subscales (Integration of sameness and differentness in society and the Autistic community and Integration into society) supports the need for more examination of the Autistic Identity Scale in different autistic samples. Specifically, it is unknown whether the covariance in the current study is due to the statistical modeling performed, or if the error covariance is present across samples and analyses. However, the overall scale performs well within the current sample, supporting Hypothesis 4.1; the Autistic Identity Scale is a plausible representation of Autistic identity as adapted from Gill (1997a).

Hypothesis 4.2. Involvement in the online Autistic community will be positively related to Autistic identity in autistic adults who prefer online communication. I examined the configural model of autistic adults who prefer online communication to support or reject Hypothesis 4.2. The covariance between involvement in the online Autistic community and Autistic identity was significant, cov=.24, SE=.08, CR=2.94, $p<.01, r=.48$. The significant and positive covariance between the two variables indicates that involvement in the online Autistic community is positively related to Autistic 
identity in the current sample of autistic adults who prefer online communication. The correlation estimate of .48 indicates a medium to strong positive relationship (Cohen, 1988) between involvement and Autistic identity. This finding supports Hypothesis 4.2.

Hypothesis 4.3. Involvement in the online Autistic community will be positively related to self-determination in autistic adults who prefer online communication. Again examining the configural model of autistic adults who prefer online communication, the covariance between involvement in the online Autistic community and self-determination was significant, $\operatorname{cov}=.09, \mathrm{SE}=.04, \mathrm{CR}=2.28, \mathrm{p}=.02, \mathrm{r}=.28$. The statistically significant and positive relationship between the variables indicates that involvement in the online Autistic community is positively related to self-determination. The correlation estimate of .28 indicates a small to medium positive relationship between the involvement and self-determination (Cohen, 1988). This finding supports Hypothesis 4.3.

Hypothesis 4.4. Autistic identity will be positively related to self-determination in autistic adults who prefer online communication. The final relationship that I examined in the configural model consisting of autistic adults who prefer online communication was significant as well, $\operatorname{cov}=.12, \mathrm{SE}=.05, \mathrm{CR}=2.69, \mathrm{p}=.01, \mathrm{r}=.47$. The significant and positive covariance between the variables indicates that Autistic identity is positively related to self-determination in the configural model sample of autistic adults who prefer online communication. The correlation of .47 indicates a medium to 
strong relationship between Autistic identity and self-determination. This significant and positive finding supports Hypothesis 4.4.

Hypothesis 4.5. Involvement in the online Autistic community will be positively related to Autistic identity in autistic adults who do not prefer online communication. I examined the comparison model consisting of autistic adults who do not prefer online communication for Hypothesis 4.5. The relationship between involvement in the online Autistic community and Autistic identity was significant, cov=.30, SE=.14, $C R=2.14$, $p=.03, r=.33$. The correlation of .33 between the variables indicates a moderately strong positive relationship between involvement and Autistic identity. Therefore, involvement in the online Autistic community is positively related to Autistic identity in autistic adults who do not prefer online communication, which supports Hypothesis 4.5.

Hypothesis 4.6. Involvement in the online Autistic community will be positively related to self-determination in autistic adults who do not prefer online communication. I again examined the model containing autistic adults who do not prefer online communication for hypothesis 4.6. The relationship between involvement in the online Autistic community was not significantly related to self-determination in the sample $\mathrm{Cov}=.004, \mathrm{SE}=.05, \mathrm{CR}=.10, \mathrm{p}=.92, \mathrm{r}=.01$. The nonsignificant relationship of .01 indicates a very weak positive relationship between the two variables. Therefore, involvement in the online Autistic community is not related to self-determination in autistic adults who do not prefer online communication. The finding rejects Hypothesis 4.6. 
Hypothesis 4.7. Autistic identity will be positively related to self-determination in autistic adults who do not prefer online communication. I next examined the relationship between Autistic identity and self-determination in the sample. The significant relationship was positive, $\operatorname{cov}=.14, \mathrm{SE}=.05, \mathrm{CR}=2.69, \mathrm{p}<.01, \mathrm{r}=.47$. The correlation of .47 indicates a medium to strong positive relationship. Hypothesis 4.7 was supported; Autistic identity is positively related to self-determination in autistic adults who do not prefer online communication.

Hypothesis 4.8. The relationships among involvement in the online Autistic community, Autistic identity, and self-determination will be stronger in autistic adults who prefer online communication than in autistic adults who do not prefer online communication. I tested the final hypothesis by examining the difference in model fit between the models tested in autistic adults who prefer online communication and autistic adults who do not prefer online communication. As reported, there was no significant difference in overall model fit between the two groups, $\chi^{2}(d f=6)=6.98, p=.32$. Therefore, invariance does not exist between groups; the proposed model (including covariance among involvement in the online Autistic community, self-determination, and Autistic identity and loadings of the four dimensions of Autistic identity) is the same for all autistic adults, regardless of whether or not they prefer online communication. However, in examining individual differences in covariances among the variables of involvement in the online Autistic community, Autistic identity, and self-determination, one relationship was nonsignificant. The nonsignificant relationship of involvement in 
the online Autistic community and self-determination was only nonsignificant in autistic adults who do not prefer online communication. Therefore, the findings partially support Hypothesis 4.8. There is no difference in overall model fit between autistic adults who prefer online communication and autistic adults who do not prefer online communication, but there is a nonsignificant relationship between involvement in the online Autistic community and self-determination in autistic adults who do not prefer online communication.

\section{Summary of Findings.}

First, I conducted descriptive data analyses (see Table 2 and Table 3), as well as tested for outliers, normality, linearity, multicollinearity, and randomness of missing data to ensure the integrity of the data and determine whether my planned significance tests were appropriate. Next, I performed a PCA to determine the data-driven weights of the multidimensional measure of involvement in the community for both the autistic and non-autistic sample (see Table 4). I then conducted an EFA of the Autistic Identity Scale to explore whether the items of the scale were a plausible representation of Autistic identity. I determined that a four factor solution containing 13 items was the best solution, and Table 5 displays the results. I also examined inter-construct correlations and determined that the lack of correlations with communication preference indicated that the planned regression analyses were appropriate (see Table $6)$. 
I then conducted all hypothesis tests. Involvement in the online Autistic community was significantly positively related to both Autistic identity (supporting $\mathrm{H}$ 1.1) and self-determination (supporting $H$ 2.1). In the regression analyses, involvement in the online Autistic community was a significant predictor of Autistic identity and selfdetermination. However, communication preference was only related to one of the four dimensions of involvement in the online Autistic community, frequency of involvement, and was not related to Autistic identity or self-determination. Communication preference ${ }^{6}$ did not moderate the relationships between involvement in the online Autistic community and Autistic identity (rejecting $\mathrm{H}$ 1.2; see 0 and Figure 2), or involvement in the online Autistic community and self-determination (rejecting $\mathrm{H}$ 2.2; see Table 8 and Figure 3). In the three-way moderated regression, the full sample included autistic and non-autistic adults, and supported that population type (autistic, non-autistic), communication preference, and involvement in the online community all significantly predicted self-determination. Self-determination was higher in non-autistic adults than autistic adults, individuals (both autistic and non-autistic) who do not prefer to communicate online, and in individuals (both autistic and non-autistic) with higher involvement in the online community. However, the interaction of the three variables was not significant (rejecting H 3; see Table 10 and Figure 4).

Next, I conducted exploratory SEM to examine a full model containing involvement in the online Autistic community, self-determination, and Autistic identity

\footnotetext{
${ }^{6}$ As described in Chapter III Review of the Literature, communication preference was dichotomized into prefers communication and does not prefer online communication (as indicated by preferring face-to-face communication or having no preference).
} 
(with the four factors of the Autistic Identity Scale) in autistic adults. I determined that the model was a good fit with the data after error variables on two of the Autistic Identity Scale factors were allowed to co-vary. I determined that the Autistic identity Scale was a plausible representation of Autistic identity (supporting 4.1), based on significant subscale paths to the latent Autistic identity variable. I next determined the model to be good fit in autistic adults who prefer online communication, and then compared the model to autistic adults who do not prefer online communication. Involvement in the online Autistic community, self-determination, and Autistic identity were significantly positively related to one another in autistic adults who prefer online communication (supporting H 4.2-4.4). In autistic adults who do not prefer online communication, Autistic identity was significantly positively related to involvement in the online Autistic community (supporting $\mathrm{H} \mathrm{4.5)} \mathrm{and} \mathrm{self-determination} \mathrm{(supporting} \mathrm{H}$ 4.7). However, involvement was not significantly related to self-determination in autistic adults who do not prefer online communication (rejecting H 4.6). Despite the overall model fit exhibiting invariance across autistic subsamples, this nonsignificant covariance indicates that the model fits less well in autistic adults that do not prefer online communication (partially supporting H 4.8; see Figure 7).

\section{Community Engagement During the Dissertation Process.}

The study findings must be interpreted through the context of the study, including the CBPR partnership that conducted the larger study and my personal experiences and interactions. Here I will discuss the current study's community 
engagement, reflect on my position as a researcher, and share community partner feedback and our reflections on the successes and shortcomings of implementing community-engaged research.

CBPR and community engagement provide greater insight into the population of interest and relevance of the work being conducted. Relevant to the present study, the six years I have worked in partnership with AASPIRE have provided me with a lens through which I have understood and defined the constructs of autism and the Autistic self-advocacy movement. Further, the partnership has allowed a deeper appreciation of the purpose, context, and implications of this study. In addition to the positive impact of community engagement in the current study, challenges existed in attempting to apply the CBPR approach in the study, which resulted in the community engagement perspective more accurately reflecting the approach of this dissertation study. The WellBeing Study successfully adheres to each of the principles of CBPR, and I was involved in the Well-Being Study since its early phases of developing research questions and the survey instrument. However, AASPIRE considers this dissertation a separate study from the Well-Being Study. This study adhered to the Gateway Project values (inclusion, respect, accessibility, and relevance) and did not adhere to all of the CBPR principles. Instead, this study used a community engagement approach. The perspectives, constructs, and methods under scrutiny in this study reflect my position of working with Autistic self-advocates, the experts of their own experiences (Yergeau, 2010). In addition to understanding how working with AASPIRE has impacted my research, I must also reflect on my own role and perspectives, which also impact my work. Next I reflect 
on my role as a non-autistic researcher conducting community-partnered work with the Autistic community, followed by feedback and reflections from community partners on the successes and shortcomings of attempting CBPR and community engagement in this study.

Self-Reflection. In order to adequately represent my perspective in this dissertation, I must reflect on my own position in AASPIRE and within the context of the research (Fine, 1992; Merrick, 1999). I became involved in disability community work in 2005, and was initially introduced to and began conducting disability research in 2006 . As a graduate research assistant, my passion for influencing communities and policies to create positive changes in individuals' lives increased and became specific to impacting the disability community. I aligned with the values of community psychology, which emphasized empowering participatory research methods with marginalized populations (Rappaport, 1977; Trickett, Kelly, \& Vincent, 1985). Through an increased awareness of the disability and Autistic self-advocacy movements, I was introduced to the disability rights slogan "Nothing about us without us." Further, through involvement in community organizations and disability research, I developed personal relationships with individuals with disabilities in addition to gaining an autistic family member. My professional and personal dedication to disability research grew, and I committed to conducting research inclusive of individuals with disabilities and relevant to the community to affect positive social change. I am interested in utilizing my skills and knowledge in research and community psychology for disability research. 
However, my commitment to the field of disability research has its boundaries due to my identity. As a non-autistic woman without a disability, my individual wellbeing does not depend on our research impacting society. To the extent anyone in the fields of disability research or justice-oriented sciences can, I am still able to remove myself from the social issues I am deeply engaged in at work or within specific advocacy contexts. While the rippling effects of a more just society would have an impact on my personal life, I likely feel less urgency in creating positive change in the lives of autistic adults than autistic adults themselves. In the context of this study, this leads to a separation existing between the Autistic community and me. With this separation comes issues of discomfort that has been described by other non-autistic researchers without disabilities (Bagatell, 2010; Oliver, 1992). I do not have the first-hand knowledge of being a part of the Autistic community, nor do I have the experiences of being autistic. Recognizing these wide differences between my research team members and me creates an internal tension of knowing my role as a non-community member academic researcher as well as my passion for involvement in research that requires me to be fully involved in the community in order to understand the context and history. Further, while I am not autistic, I still relate to fluctuating disempowering contexts in my life as a woman, a student, and as a non-member of the community with which I directly do research (Kidney \& Stack, 2012).

Despite my struggle to find a voice within Autistic research, I feel as though my self-reflection further encourages me to engage in CBPR research with the community. 
Understanding the challenges I face as a member of a collaboration aimed at positive social change helps me work through the difficult feelings and appreciate the accomplishments of the team. Further, I strongly believe in work that pursues the interests of the community, seeks to share information with a broader audience about the strengths of the community, and generates new ideas and questions about the community. I feel as though such research can impact society's views about who autistic people are, and how autistic people should be viewed and treated as individuals and in policies and systems. Further, research that sheds positive light on the history and positive outcomes of involvement in the online Autistic community has the potential to change stereotypes about what it means to live a meaningful life and be a part of a meaningful community that is accepted and embraced for its diversity. Findings could influence education, policies, programs, and families to acknowledge, encourage, and provide resources for the fostering of peer support and community. Findings from this study suggest that the Internet may be the best vehicle for fostering such a network of support and community.

Self and community reflections on community involvement. In addition to reflecting on my own position as a researcher, it is also necessary to reflect on the actual process of the conducting the study. Here I discuss my own reflections on the process of my dissertation as well as feedback and reflections from community partners on their experiences of involvement with my dissertation. 
This study utilized data from the AASPIRE Well-Being Study, which applied a CBPR approach. I utilized a community-engaged approach for the specific steps of this dissertation. However, I originally intended to conduct the study with a CBPR approach as well. The study fell short of CBPR due to challenges in relationship building and consistent communication, aligning more closely with a consultation-level of community engagement (CDC, 2011). To fulfill an important aspect of CBPR and community engagement-the cyclical and reflective nature of the process-it was necessary to acknowledge and further reflect on the challenges of this dissertation in involving the community in the study process, including seeking and reporting on the perspectives of community partners on their involvement in my dissertation. Reflecting on the challenges of the process may also contribute to the CBPR literature, addressing a gap that exists in the literature of describing somewhat negative or challenging experiences of CBPR.

To receive feedback from community partners on my dissertation process, I held meetings with the three remaining activity community partners on my study. I asked questions about their level of involvement with my study, and their feelings regarding my adherence to the AASPIRE and CBPR principles of community involvement. Because I met with each partner separately, I also asked partners if they agreed with comments that I shared from other community partners. I organized my reflections and their feedback here.

The major shortcoming of the current study in attempting CBPR principles lies in creating equal partnership and ongoing bidirectional communication among team 
members. While I did check-in with AASPIRE partners at each stage of my dissertation, after my research aims and questions were developed based on feedback from a team meeting, partners had no active involvement in the study beyond providing approval and feedback of the major steps of the project. The CBPR principle of identifying and building upon community strengths, resources, and relationships in the community (Israel et al., 1998) was not met for my dissertation study. While only providing feedback when it was requested, community partners were not contributing their skills to the study. This also relates to the principle of CBPR that emphasizes collaborative partnerships between all team members in all phases of the research. By understanding the skills of community members, we may have improved my dissertation study by working together. For example, a more detailed understanding of the study findings may result in a greater depth of community perspective in the discussion and findings. Further, community partners may have more ideas and community-level perspective on the implications of the study if they were more involved.

Reflecting on how community partners were minimally included in the process, I believe I could have integrated more engagement with community partners while still leading the study. That is, rather than holding one-on-one or group meetings for each of the major milestones of the study (brainstorming ideas, approval of my dissertation idea, approval of my proposal, presentation and interpretation of findings, etc.), I could have held on-going meetings to discuss and receive feedback on individual research questions, findings, and interpretations. More frequent meetings would provide community partners with a greater depth of understanding of my study, which would 
then result in greater depth of community perspectives throughout my dissertation. For example, perhaps if the team had a stronger understanding of some of the complex analytic findings of this dissertation, they would be able to provide a community perspective that I have not considered in the discussion. Community partners all agreed that I should have kept in more constant contact, even if it was monthly progress updates. One community partner suggested that I could have asked for help with minor tasks to keep people engaged, such as help finding relevant literature or website sources.

Sometimes during my dissertation process, I would ask for AASPIRE approval for an idea or document, and community partners would express frustration with my technical presentation. One community member felt as though I did not consider her advice for how to best communicate with each team member, which would improve relationships and communication. A number of times community partners would provide approval without completely grasping the meaning of the document based only on not seeing anything offensive to the Autistic community. I again believe that this process could have been improved with more ongoing communication about ideas, which would result in relationship-building and greater understanding of communication styles so I could develop materials and presentations that are more digestible to the AASPIRE team. While CBPR emphasizes a cyclical process of involvement where ideas are developed and revised and ideas are reflected upon and improved, the community engagement of my dissertation process involved my ownership and presentation of materials and receiving feedback that was incorporated 
into my study but rarely revisited by community partners. One community member noted that she was unsure whether her advice and feedback was taken into consideration due to a lack of communication between feedback meetings and emails.

The principle of transferring knowledge and power (Israel et al., 1998) was impossible given the lack of communication and incomplete grasp of my perspectives, aims, and findings of the study. The community partners remained reliant on my communication to have any mastery, power, or leadership in the study. A negotiation of the ownership should have occurred between my dissertation and the knowledge gained from the dissertation process. That is, while I was required to lead the academic aspects of the dissertation and write and present the study, we could have established early on what the community partners' roles may be to stay involved, have an impact on the study, and be impacted by involvement through learning new skills or content.

This dissertation study took place over a three year period, during which some AASPIRE team members switched jobs, graduated school, went back to school, and experienced many non-professional life changes. Further, the way AASPIRE structured team membership and communication for its different studies changed over time, with increasing separation between studies. Individuals on the AASPIRE teams have moved on from AASPIRE, and new members of the team have joined AASPIRE. Changes in team members' individual lives in addition to structural changes to AASPIRE have lead to fewer individuals being involved in the Well-Being Study and therefore my dissertation study. Since data collection ended in 2012 and dissemination of findings was limited until I finished this dissertation, the few busy members of the Well-Being Study team 
may have decreased their interest in involvement to put their attention to other AASPIRE studies or unrelated work. Ongoing engagement with partners around the study may have created greater interest in this dissertation to reflect the strong interest present in earlier stages of the Well-Being Study. One community partner indicated I could have shown better leadership and control over the process by addressing challenges in the project (e.g., more initiative to shorten the timeline) and engaging community partners (e.g., continued attempts at communication and engagement when I was unsuccessful).

Given the minimal engagement and long time frame of the dissertation study, community partners may not be aware of my dedication to the study and my passion for the promotion of research that benefits the Autistic community. Without strengthening my relationship with community partners, it was impossible to build an understanding of each of our relationships to the research. Community partners all indicated that they felt that their role was more of a consultation or advisory role than a community partner. They felt as though engagement outside of my specific dissertation project (e.g., involvement in broader AASPIRE conversations and meetings) would have deepened relationships and understanding of one another. Fostering more meaningful relationships with community partners would make engagement, understanding, and collaboration easier and more successful.

Recognizing the shortcomings of my CBPR approach, I did engage AASPIRE partners during major phases of the dissertation study. Additionally, CBPR principles were utilized for the larger Well-Being Study, so the benefits derived from the use of 
CBPR in determining overall study aims, methods, measures, and recruitment still apply to this study. While I cannot claim that CBPR was successfully applied through all stages of this dissertation, the study was conducted with engagement with community partners. The literature describes participatory action research, a research approach with community-engaged components similar to CBPR, as consisting of levels of engagement with the disability community ranging from engaging an advisory board at the lowest level to having true partnerships in the research team with community members (Balcazar, Keys, Kaplan, \& Suarez-Balcazar, 1998). Considering those levels of engagement, this dissertation falls short of complete partnership but is higher than the lowest levels due to my participation with the research group since the Well-Being Study's beginning through the approval of all stages of my study. Therefore, the findings of the current study should not be interpreted as coming from a purely CBPR approach, but as a community-engaged approach that attempted CBPR.

Finally, the dissertation process does not have to end with the submission of a final document. CBPR's final principle involves disseminating findings to promote knowledge and benefit the partners and community. The dissemination of findings from this dissertation may involve community partners as equal partners and engage them in ways this dissertation fell short.

These reflections may allow for a richer understanding of the perspective and context of the study, particularly the interpretations of findings in the Discussion section. Greater involvement of the community partners may lead to greater 
understanding of why particular findings may exist. Next, the Discussion section includes interpretations of findings, study strengths and limitations, and implications. 


\section{CHAPTER VI}

\section{Discussion}

This study aims to explore factors related to involvement in the online Autistic community, an under-studied group, to promote Autistic research and knowledge about the community. This study examined the relationships between involvement in the online Autistic community and Autistic identity and between involvement and selfdetermination, and whether communication preference ${ }^{7}$ moderated both of the relationships. The study also examined whether the moderation of communication preference varied by the group type (autistic, non-autistic) in the relationship between involvement in the online community and self-determination. Lastly, the study examined the relationships among involvement in the online Autistic community, Autistic identity, and self-determination in autistic adults, and compared the relationships among variables in individuals who do and do not prefer online communication.

The study hypothesized a positive relationship between involvement in the online Autistic community and both Autistic identity and self-determination, that communication preference moderates the relationships, and that group type (autistic, non-autistic) moderate the relationship between involvement in the online community and self-determination. The cross-sectional sample was relatively narrow and small considering the number and type of exploratory analyses, which should be considered in

\footnotetext{
${ }^{7}$ Communication preference is dichotomized in the current study as "prefers online communication" or "does not prefer online communication," which includes preferring face-to-face communication and no preference for communication.
} 
interpreting findings. Despite sample and analytical limitations, analyses supported positive relationships between involvement in the online Autistic community and both Autistic identity and self-determination. Analyses did not support communication preference as a moderating variable in the relationships, nor population type (autistic, non-autistic) as a moderating variable between involvement in the online community and self-determination. The study also hypothesized the plausible representation of the Autistic Identity Scale for Autistic identity, and that involvement in the online Autistic community, Autistic identity, and self-determination are positively related in autistic individuals who do and do not prefer online communication, but are stronger in those who do prefer online communication. Analyses supported that the Autistic identity Scale is a plausible representation of Autistic Identity. While analyses supported positive relationships between involvement in the online Autistic community, Autistic identity, and self-determination in autistic adults who prefer online communication, the relationship between involvement in the online Autistic community and selfdetermination was non-significant in autistic adults who do not prefer online communication, partially supporting the hypothesized comparison.

\section{The Autistic Identity Scale}

Prior to hypothesis tests in the current study, I explored the factor structure of the Autistic Identity Scale using EFA. Beyond analysis of a subset of the current data (Kidney, 2012), the current study is the first to utilize and examine the Autistic Identity Scale that was adapted from Gill's (1997) Disability Identity Scale. Prior to the EFA, the 
factor structure of the scale was undocumented. Examination of the psychometric properties of the scale was necessary for valid results of hypothesis testing, as well as for contributing to the identity literature by examining a scale with a new population. The EFA resulted in a 13 -item scale since I removed five items from the original scale due to poor performance in the EFA and low subscale internal consistency reliability. The revised scale measures each of the four dimensions of the Autistic identity theory as adapted from Gill (1997), including integration into society at large, integration into the Autistic community, internal integration of sameness and differentness with society and the Autistic community, and integration of feelings about self and presentation of self. The revised scale shows good reliability overall, with subscales showing acceptable reliability.

In addition to the EFA, I examined the structure of the revised Autistic Identity Scale in autistic adults as a component of the structural model containing involvement in the online Autistic community, self-determination, and Autistic identity (containing the individual factors). The factors positively and significantly loaded onto the Autistic identity latent variable, and were part of the overall model's good fit. The internal consistency reliability and SEM findings indicate that the Autistic Identity Scale is a plausible representation of Autistic identity as adapted by Gill's (1997) disability identity theory.

The exploratory psychometric analyses conducted in this study are preliminary results within a relatively small and homogenous sample. Despite the limitations of the sample, the plausibility of the revised scale within Gill's (1997) theoretical framework 
shows the conceptual similarities between disability identity and Autistic identity. The Autistic community may be considered an outgrowth of the disability community, with many overlapping values and advocacy goals (Ward \& Meyer, 1999). Further, Gill's disability identity theory has been applied to other marginalized identities such as gay and lesbian identity (Cramer \& Gilson, 1999), showing the wider utility of the theory. Individuals belonging to marginalized groups often find communalities with one another apart from the majority of the population (Fine \& Asch, 1988). This has been qualitatively examined in Autistic individuals who experience "othering" themselves from the dominance and power of the majority of the population and seeking other Autistic individuals to foster a positive sense of self (Brownlow, 2007). While individuals have unique experiences of contextual identity development, perhaps Gill's theory taps into universal experiences of developing identity within any marginalized group. Not all autistic individuals may consider themselves part of the Autistic community due to differing values, interests, or support from other communities. Therefore, it is unlikely that individuals who are not involved will exert high Autistic identity, since the Autistic community advocates that autism should be viewed in a strengths-based manner.

However, further testing of and improvements to the Autistic Identity Scale are essential due to the internal consistency reliability alphas of subscales being slightly lower than a good alpha level and derivation of the revised scale from a single sample. In the current sample, each of the subscales has an alpha between .70 and .78, indicating acceptable but not good reliability (Cronbach, 1951). This indicates slightly higher measurement error and slightly lower inter-item correlations within subscales, or 
that each subscale may not accurately measure the same latent trait (Tavakol \& Dennick, 2011). Since the alphas were not unacceptably low and the results of the EFA and SEM indicated a plausible representation of the dimensions of Autistic identity, future research may consider use of the revised scale developed in this study. However, adding items to subscales that tap into each of the dimensions may increase the subscale alphas (Tavakol \& Dennick, 2011), particularly in the subscales that contain two or three items. For example, adding items to the dimension of "Integration into society" such as "Society is better because of the contributions of people on the autistic spectrum" would increase the number of items in the subscale and may increase the subscale alpha.

Other improvements to the scale could increase the subscale alphas and as well. The subscale "integration into the Autistic community" contained the highest number of items (five items), yet had the lowest alpha (.70). Future scale work may improve this subscale by re-examining existing items. The subscale is defined as overcoming negative feelings about being autistic to find relationships with other autistic individuals rewarding and important. Perhaps the item "I feel good about people on the autistic spectrum" can be improved with more specificity, such as "I feel good about the relationships I have with people on the autistic spectrum." Greater specificity about what exactly the item is asking may allow autistic adults responding to the item have a clearer understanding of the meaning of the question and therefore respond more accurately. 
Additionally, two items in this subscale loaded into another factor in the EFA ("integration of feelings about self and presentation of self"), but were determined to be more closely aligned with this factor. Despite the alpha of the scale decreasing when these items are removed, the items may be the potential source of improvement if modified. For example, the item "My future is tied to the future of other people on the autistic spectrum" may cause poor performance in individuals who find vague or abstract concepts difficult to understand, which some autistic individuals experience. Perhaps modifying the item to "Events that are important to the Autistic community personally impact my life" would improve item performance and increase the subscale alpha by tapping more closely into the closeness of individuals to the Autistic community. Future research may also improve the other item in the subscale that loads onto two subscales, "I have a strong sense of belonging to the Autistic community" with less abstract wording. Perhaps modifying the item to "I have close relationships in the Autistic community" would improve the item and subscale alpha.

Lastly, I removed five items from the original scale due to poor performance in the EFA (i.e., mixed results of factor loadings and confusing factor distinctiveness) and because the items decreased the scale and subscale internal consistency reliability. In addition to performing poorly in the scale, the removed items ("Overall, people on the autistic spectrum are considered valuable by others," "Most people consider people on the autistic spectrum, on average, to be less effective than people not on autistic spectrum," "In general, others respect people on the autistic spectrum," "In general, others think that people on the autistic spectrum are unworthy," and "In general, others 
think that people on the autistic spectrum are unworthy") all ask about what the respondent thinks is others' perspectives on being autistic rather than asking about the perspective of the respondent. No items that were included in the 13 -item scale contained this perspective. The exclusion of items that ask about perceptions of others may indicate that Autistic identity is not dependent on how others think about autism. Since Autistic identity is characterized by alignment with a marginalized community, it may be important to reject how others perceive the worthiness of autistic individuals. The oppressive majority's opinion may be irrelevant when developing an identity outside of majority expectations or standards of what a person should be like. The poor performance of the items asking for others' perceptions may also align with characterizations of autism that indicate autistic individuals find understanding others' feelings or emotions difficult, known as theory of mind (Baron-Cohen, Leslie, Frith, 1985). However, one should take caution in interpreting the poor performance of the items as respondents' inability to understand the perspectives of others. Some literature criticizes theory of mind as ignoring complexities of how individuals empathize and individual variation (Cohen-Rottenberg, 2012; Gernsbacher, 2005).

While Gill's (1997) Disability Identity theory discusses integration into society and integration of society and the disability community, these dimensions do not specifically include how individuals perceive others' opinions. The revised scale excludes the perception individuals have of others' opinions, which may make the scale align more appropriately with the theory. No published work includes information on an exploration of the scale within disability identity literature; therefore, it is unknown 
whether these five items perform well in the Disability Identity Scale (Gill, 1997). Further research should determine whether these items are uniquely inconsistent in Autistic adults due to the Autistic community's rejection of others' perspectives, or are also poorly performing items in other populations. If the items do fit within disability identity, the unique experiences of Autistic identity or characteristics of Autistic individuals should be further examined.

The limitations of the current study analyses-including a homogenous sample utilized for multiple exploratory analyses - create the need to evaluate the psychometrics with additional samples of autistic adults. Additionally, the revised scale may also have unique relevance for autistic adults. Therefore, research is needed to examine the scale's properties for adults with other disabilities. Despite recommended improvements to items and testing on the psychometric properties of the scale, the revised Autistic Identity Scale is a plausible representation of Autistic identity based on acceptable internal consistency reliability (Cronbach, 1951), theoretical alignment with the theory of disability identity (Gill, 1997), and good model fit and significant factor loadings in the SEM. Therefore, I utilized the revised scale in hypothesis testing for the current study, and recommend the scale for inclusion and further examination in future studies examining Autistic identity. The current study utilizes the overall study mean for hypothesis tests, and does not examine relationships between the Autistic identity subscales and other variables included in the SEM in order to limit the number of parameters in the analysis of a relatively small sample. However, examining whether Autistic identity subscales are related to self-determination, involvement in the online 
Autistic community, or communication preference should be considered in future research.

In addition to the psychometric properties explored in the current study, I also recommend the revised scale for further use due to the thoughtful selection, review, and edits to the scale by the CBPR team. Community partners agreed that the revised Autistic Identity Scale is a valid representation of Autistic identity. I presented the scale items and welcomed questions, comments, or suggested changes to the items. No partners suggested any changes or arguments against using the scale or subscales, and agreed that the data-driven EFA technique provided plausible dimensions. When presented with the poorly performing items recommended for approval, community partners agreed that they should be taken out of the scale, suggesting the third person perspective of the scale (others' opinions of autistic individuals) may be inappropriate for a person who is gauging their own identity. Further use and improvement of the scale may significantly contribute to the literature on Autistic identity and inform how teachers, service providers, and advocates approach identity development and pride in Autistic individuals.

\section{Involvement in the Online Autistic Community and Autistic Identity}

The first relationship that the current sample data supported was that involvement in the online Autistic community is significantly positively related to Autistic identity. A correlational analysis supported the relationship, within the regression model containing Autistic identity regressed on involvement in the online 
Autistic community and communication preference, within the SEM containing all autistic adults, and within the comparison models of autistic adults who prefer and do not prefer online communication. This supports findings of the analysis of a subset of the current sample that found a positive relationship between involvement in the online Autistic community and Autistic identity (Kidney, 2012). However, the current study utilized a multi-dimensional composite variable that likely provided a better measure of involvement in the online Autistic community beyond what was previously used-- the single item indicator of importance of involvement in the online Autistic community. The composite variable utilized importance of involvement in addition to other dimensions that a PCA determined were contributors to the variable. In addition to analyses within the current sample, previous research in general populations indicates that use of the Internet contributes to an individual's identity development (Turkle, 1995), and that involvement in a social context is related to identity development (Hendry, 1983). Previous research also indicates the Internet use and online socializing have shown to increase social capital and friendships among (Boase et al., 2006; Katz et al., 2004), which in turn impacts quality of life (Kraut, Kiesler, Boneva, Cummings, \& Helgeson, 2002; Putnam \& Miller, 1995). Further, research shows that participation in social movements involves the development of a personal identity around the community and a sense of self-realization (Gamson, 1992; Teske, 1997).

Specific to the online Autistic community, autobiographical accounts, online discourse, and interviews demonstrate a personal and community identity that develops through involvement in the online Autistic community (Bagatell, 2007, 2010; Brownlow 
\& O’Dell, 2006; Burke, Kraut, \& Williams, 2010; Davidson, 2008; Silverman, 2008;

Yergeau, 2010). By developing a multi-dimensional measure of involvement in the online Autistic community and supporting a relationship to Autistic identity, the current study supports information that has previously only been supported qualitatively in autistic adults and in a subset of the current sample (Kidney, 2012). The current study supports the relationship between involvement in the online Autistic community and Autistic identity. Beyond immediate implications of this study finding, distal implications exist. Since positive outcomes such as pride and well-being have resulted from identity development (Berkman, Glass, Seeman, \& Brisette, 2000; Gill, 1997; Hendry, 1983; Wandersman \& Florin, 2000), the positive development of Autistic identity in individuals involved in the online Autistic community may contribute to similar positive outcomes. Therefore, education and resources for autistic individuals should be provided to increase involvement in the online Autistic community in order to promote such positive outcomes.

This result may be due to Autistic individuals finding commonality with one another apart from those not experiencing marginalization (Fine \& Asch, 1988) and experiences with self-advocacy efforts to address marginalization. Additionally, autistic adults with strong Autistic identity may be drawn to participate to a greater extent in the online Autistic community. According to Gill's (1997) theory of disability identity development, identity is strengthened by integration into society at large ('coming to feel we belong'), integration into the disability community ('coming home'), internal integration of sameness and differentness with society and the disability community 
('coming together'), and integration of feelings about self and presentation of self ('coming out'). The current study applied Gill's theory to autistic adults, and found the measure to be a plausible representation of the theory. Therefore, the online Autistic community may serve to promote positive Autistic identity by creating social relationships and support to feel integrated in the Autistic community and by educating and working together for social action to internally integrate feelings of sameness and differentness of society and Autistic community. Involvement in Autistic self-advocacy efforts and fostering relationships with other Autistic adults dynamically increases as identity with the community increases.

However, post-hoc correlational analyses indicated that involvement in the online Autistic community is not related to integration into society or integration of feelings about self and presentation of self. The nonsignificant relationship with integration into society suggests that higher involvement in the online Autistic community may lead some individuals to an Autistic identity that is marginalized and separate from society. This may be a result of Autistic people spending more time and having more of a connection with their Autistic peers, and therefore do not feel equally connected with society as a whole. Autistic individuals who feel less integrated into society may seek solace and find great importance and pleasure in the online Autistic community. However, since involvement in the online Autistic community is not negatively related to involvement in society, involvement in the online Autistic community does not have a statistically negative impact on individuals' connection to society. The finding that Autistic individuals may socialize more amongst peers in the 
online Autistic community and it does not negatively impact involvement in society may challenge characterizations of autism that define autistic individuals' differences in socializing as an impairment (American Psychiatric Association, 2013). Instead, a like group of individuals may be providing greater support and community to this typically marginalized population, similarly to the function of support provided by communities of peers in other minority groups (Brownlow, 2007; Fine \& Asch, 1988).

The nonsignificant relationship of involvement in the online Autistic community and integration of feelings about self and presentation of self may be a result of the anonymity and inclusiveness of the Internet. This dimension of Autistic identity concerns Autistic individuals being comfortable with how others perceive them as autistic. However, the Internet allows everyone to only disclose what they want disclosed about their identity. While embracing being autistic seems like a logical relationship with Autistic community involvement, perhaps without being forced to be "out" as autistic online the relationship does not exist.

Additionally, the powerful tool of the Internet provides the context from which the relationship between involvement in the online Autistic community and Autistic identity exists. Research shows that the Internet may be a tool for strengthening aspects of the self-concept through becoming aware of a new identity outside that of the offline world (Amichai-Hamburger, 2005; Suler, 2004). Self-expression, self-reflection, and dialogue may allow individuals to explore dimensions of themselves uniquely visible in the online Autistic community. Autistic individuals may also use the Internet as a way for autistic individuals to learn about themselves through seeking others' experiences of 
being autistic, as well as to refine how they think and communicate about being autistic. Identity development occurring in the online Autistic community likely carries into other contexts as well, with stronger Autistic identity impacting involvement in the Autistic community offline, meeting Autistic peers online then meeting them offline, and becoming involved in advocacy efforts that are both online and offline.

Autistic individuals may find commonalities that lead to shared identities based upon being Autistic through online contact with others experiencing the same societal barriers. Rejection of person first language by Autistic advocates is one example of asserting and shaping identity as an Autistic person as central to a person's being (Silverman, 2008), and likely reflective of a strong sense of Autistic identity. Brownlow (2007) points out that identifying with the Autistic community is central to involvement with the community, and that identity is a complex dynamic construction for every individual involved. The complexity of identity may be attributed to the online documentation of autobiographical accounts of the unique strengths and challenges, perspectives, and knowledge that come with autism (Davidson, 2008), creating multiple subjective understandings of autism and the Autistic self. Perhaps participation in the online Autistic community increases Autistic identity through self-reflection and reading and sharing experiences around being an Autistic adult. A post-hoc test rejected the relationship between Autistic identity and years involved in the online Autistic community. The nonsignificant finding places more emphasis on the quality or intensity of such relationships and experiences within the community, rather than duration of time, in developing Autistic identity. Further, individuals that have been a part of the 
online Autistic community longer may have withdrawn somewhat due to negative experiences with cyber bullying, or making connections online then continuing relationships offline.

The positive relationship between involvement in the online Autistic community and Autistic identity may provide rationale for education, training, and support for autistic individuals to have access to computers and Internet. Access to the online community may allow them to seek out the information, people, or services that can assist them in making critical life decisions, which may be lacking in their lives (Barnard, Harvey, Potter, \& Prior, 2001). A feeling of pride and positivity around Autistic identity may result from the resulting knowledge and access, including validating the feelings of differentness felt by some autistic individuals (Silverman, 2008). A positive identity development is related to other positive outcomes, including self-determination.

\section{Involvement in the Online Autistic Community and Self-Determination}

The study also supports a significant positive relationship between involvement in the online Autistic community and possible indicators of self-determination. Specifically, the data support a significant positive relationship between involvement in the online Autistic community and self-determination in the correlational analysis, in the regression model containing self-determination regressed on involvement in the online Autistic community and communication preference, the regression model also containing population type (autistic or non-autistic), in the SEM containing all autistic adults, and in the SEM containing autistic adults who prefer online communication. No 
study has examined this relationship; however, Ryan and Deci's (2000) theory of selfdetermination posits that a supportive environment increases the psychological needs that compromise self-determination (autonomy, competence, and relatedness). The online Autistic community is a context that supports communication and the fostering of supportive community (Brownlow \& O’Dell, 2006; Kidney, 2012). The positive relationship between involvement in the online Autistic community and selfdetermination further suggests the supportiveness of the online Autistic community context in increasing autonomy, competence, and autonomy in autistic adults. The association between involvement in the online Autistic community and selfdetermination may be interpreted as individuals with higher self-determination seeking and becoming more involved in the online Autistic community more than individuals with lower self-determination. The relationship may be transactional, with selfdetermination positively impacting involvement in the online community, and involvement in the community increasing self-determination. As the study is crosssectional, the directionality of the association is unknown.

This result may be due to the online Autistic community providing resources and support that promote self-determination. Autistic adults are members of a marginalized community that often lack the knowledge or opportunities to exhibit self-determination. Autistic individuals involved in the online Autistic community may be involved to foster relationships with other Autistic individuals, understand and relate to others' experiences, and become active in Autistic advocacy efforts. Such activities may inform self-determined behavior and choices, such as by exposing autistic individuals of their 
rights and ways to assert rights. Connecting with other Autistic individuals may provide individuals with the confidence and tools to lead a more self-determined life by allowing individuals to feel belonging, membership, and likeness with others through their shared experiences. A transactional relationship between involvement in the online Autistic community and self-determination likely exists. Involvement in the online Autistic community may also be a result of a person with higher self-determination. For example, a person who has higher self-determination may have the computer and Internet skills and knowledge of the online Autistic community in order to seek out and become involved in the online Autistic community. Involvement may increase selfdetermination while self-determination drives increased involvement in the community. Post-hoc analyses supported that self-determination is significantly positively related to sense of community and importance of involvement. However, selfdetermination is not significantly related to years of involvement or frequency of involvement. This finding suggests that the subjective judgments of the quality of involvement in the online Autistic community are more important in the relationship to self-determination than the actual time spent with the community. Perhaps only a strong feeling of connection with the community bolsters self-determination, rather than greater amounts of time spent in the community. The frequency of involvement may also be related to unfamiliarity with computer/Internet technology or navigating the online community, which logically would not relate to self-determination since higher frequency would mean lower autonomy and competence navigating the community. Years of involvement, measured without the other involvement 
dimensions, does not consider whether a person has been minimally involved over a long period of time, or whether involvement was consistent over time. Additionally, the PCA indicated that years of involvement was the least important indicator of involvement in the online Autistic community, with the smallest percentage of the composite variable compromising years of involvement. Therefore, a nonsignificant relationship between years of involvement and self-determination should be interpreted with caution. However, like the relationship between involvement in the online Autistic community and Autistic identity, this finding could suggest that the length of time involved in the community is not as important as feelings of belonging and connection to developing self-determination.

Post-hoc analyses also indicate that involvement in the online Autistic community is only significantly related to one of the three dimensions of selfdetermination, relatedness, but is not related to autonomy or competence. This finding, when considered with the post-hoc findings that self-determination is only related to the subjective qualities of involvement in the online Autistic community, suggests that the relationship between variables may be based solely on the dimensions pertaining to connections with other Autistic individuals in the community. That is, the online Autistic community may be mostly for the purpose of fostering relationships and a sense of belonging with a community. While information can be sought out online that would lead to higher autonomy and competence (e.g., tools for acquiring employment or life skills, encouragement that leads to confidence in controlling and directing one's life), the relationships with others are the most valuable aspect of involvement for Autistic 
individuals, and result in a shared connection and relatedness with others. The strong associations between subject and relationship-focused aspects of the variables suggest the need for further research, particularly surrounding the measurement of selfdetermination, to identify whether involvement in the online Autistic community positively impacts other dimensions of self-determination.

The data supported positive relationships among involvement in the online Autistic community, Autistic identity, and self-determination. A further implication of the current study is that findings may provide further rationale for supporting autistic individuals in learning, accessing, and utilizing the Internet. The current study findings can potentially inform promotion of policies and support/services that allow autistic individuals to seek out their community online for greater self-determination in order to improve individuals' quality of life and life satisfaction (Cagle, 2006; Lachapelle et al., 2005; Nota, Ferrari, Soresi, \& Wehmeyer, 2007). Previous research shows that selfdetermination is a valuable skill for individuals with disabilities that can be taught in school and home (Malian \& Nevin, 2002). The current study may provide support for one method for potentially increasing self-determination in autistic individuals. Additionally, previous research on self-determination in autistic individuals is scarce and focused on children (Field \& Hoffman, 1999). The current study supports the link between involvement in the online Autistic community and self-determination in autistic adults, which may be viewed as a distal outcome for earlier education or for an opportunity for adults to become involved in the community. 


\section{Self-Determination in Autistic and Non-Autistic Adults in the Online Community}

While true comparisons of autistic and non-autistic individuals should be achieved only through population-based studies, this study explored sample comparisons in self-determination. A post-hoc test supports that self-determination is higher in non-autistic individuals than autistic individuals in the current sample. Findings also support that autistic adults and non-autistic adults do not differ in their level of involvement in online communities; therefore the significant difference in selfdetermination is attributed to population type alone rather than an interaction of variables. This supports a previous study finding that indicates autistic students exhibit lower self-determination than non-autistic peers (Wehmeyer et al., 2010).

The relationship between population type and self-determination and the significant difference between self-determination in autistic and non-autistic adults may be due to autistic adults experiencing oppressive contexts, a lack of knowledge about ways to control one's own life, and a lack of opportunities to develop and exhibit selfdetermination. The present study measured self-determination in a sample of autistic adults who are involved in the online Autistic community, which may be a biased sample with higher self-determination compared to the general population of autistic individuals who may not have the same levels of opportunity, support, skills, or autonomy. Despite the involvement of the autistic sample with a supportive community, self-determination was still higher in the non-autistic sample, perhaps due to the issues of continued marginalization and oppression. 
Further, perhaps autistic individuals' self-report of self-determination illuminates the internalized oppression of some individuals in the Autistic community. Autistic individuals may view themselves as less self-determined due to differences in face-toface communication skills, as such communication is generally viewed as superior (Bell, 2009; Shaffer et al., 2000). While the Internet may promote self-determination and bypass communication difficulties, it is possible that autistic individuals continue to frame their strengths within society's commonly accepted viewed of autonomy, competence, and relatedness.

Previous literature links Internet addiction and problematic Internet use to autistic traits, depression, anxiety, and schizotypal traits (Romano, Osborne, Truzoli, Clubley, 2001). While the premise of such studies is questionable due to their characterization of high Internet use as negative, the current study findings may relate to studies that indicate that high levels of Internet use may not be entirely beneficial to autistic individuals. Future studies should determine how autistic adults spend their time online and whether different amounts of time spent online (moderate use versus heavy use) differently impact positive outcomes. Additionally, controlling for or matching autistic adults to non-autistic adults based on type of Internet use may provide more information on the outcomes of the study.

However, the significant difference supported in the current sample between non-autistic and autistic individuals on self-determination may be due to sampling or measurement biases. Since most recruitment occurred via disability and autistic-related websites and pages, the non-autistic sample may be more likely to be in positions of 
supporter, advocate, or family member. Responding to questions about selfdetermination within a context of Autistic research may create a bias in how the sample viewed their self-determination. The current study also utilized a theory and measurement of self-determination for general populations (Ryan \& Deci, 2000). Perhaps the significant difference is a result of autistic adults experiencing selfdetermination differently than non-autistic adults, making the comparison of the groups less valid. Overcoming unaccommodating environments and navigating interpersonal supports may be key features of autistic self-determination that are not considered in a theory or measurement of self-determination in non-autistic individuals.

In addition to study limitations, the results should be interpreted cautiously when considering the practical significance of the small difference in self-determination between autistic $(M=2.64, S D=.38)$ and non-autistic adults $(M=2.83, S D=.38)$. While the analysis supports statistical difference between the means, a difference of 0.19 on a 5point Likert scale may not be meaningful in observing self-determination in individuals. Similarly, the statistically significant difference in self-determination between individuals who do not prefer online communication $(\mathrm{M}=2.79, \mathrm{SD}=.39)$ and individuals who do prefer online communication $(M=2.70, S D=.40)$ may not have practical significance. $A$ difference of 0.09 in the mean scores of self-determination would likely not have an observable difference outside of this scale and analysis. The practical significance of these tests should be considered when interpreting differences in self-determination in the current study. 
Despite the finding that self-determination was higher in non-autistic adults than autistic adults, I recommend further research to determine if other factors contribute to the significant difference, or whether the difference in the current study is due to sampling bias or the validity of the self-determination instrument across samples. Since the current study supports other positive relationships with self-determination, including involvement in the online Autistic community and Autistic identity, the difference in autistic and non-autistic adults in self-determination provides further rationale for autistic adults to have access to their peers online. Additionally, findings should not be interpreted as indicating level of functioning. Limitations to the sample, measurements, and analyses exist, particularly the lack of population-based sampling in order to make a sufficient comparison of autistic adults to non-autistic adults. The sample contains a relatively homogenous group of autistic adults which limits a comparison across all autistic adults to non-autistic adults. Since the sampling procedures focused primarily on the autistic population, findings from the non-autistic sample may not be generalizable as well. Further, the exploratory analyses utilizing the study's small cross-sectional sample should be replicated in additional larger samples to confidently interpret confirmatory findings.

\section{The Influence of Communication Preference in the Online Community}

The findings in this cross-sectional sample indicate that communication preference does not statistically impact the relationship between involvement in the online Autistic community and Autistic identity or the relationship between involvement 
in the online Autistic community and self-determination. Communication preference also does not influence whether population type (autistic or non-autistic) influences the relationship between involvement in the online community and self-determination. Despite non-significant regression findings concerning communication preference, the SEM comparison model between autistic adults who prefer online communication and do not prefer online communication supports a significant impact of communication preference on the relationship between involvement in the online Autistic community and self-determination, since involvement and self-determination are significantly related in the model containing autistic adults who prefer online communication, but nonsignificant in autistic adults who do not prefer online communication. Further, posthoc tests support that individuals (autistic and non-autistic) who do not prefer online communication have higher self-determination than individuals who prefer online communication. Additionally, individuals who prefer online communication have significantly higher involvement in the online community (autistic and non-autistic) than individuals who do not prefer online communication.

Autistic Identity. The findings of the current study concerning communication preference support that a preference for online communication does not directly impact whether autistic adults involved in the online Autistic community have higher Autistic identity. That is, all autistic adults who have higher involvement in the online Autistic community have higher Autistic identity than autistic adults who have lower involvement in the online Autistic community. The two-group SEM containing the relationship between involvement in the online Autistic community and Autistic identity 
in both autistic adults who prefer online communication and who do not prefer online communication provides more information on the lack of impact of communication preference on the variables. The positive relationship existing in both communication preference groups and fitting the SEM suggests that communication preference does not have an impact on involvement in the online Autistic community and Autistic identity.

Specifically, the regression model provides support for the way in which communication preference does not influence the relationship between involvement in the online Autistic community and Autistic identity; as involvement in the online Autistic community increases, Autistic identity increases equally in autistic adults who prefer online communication and in autistic adults who do not prefer online communication. However, the SEM provides support that the data in both groups of autistic adults fit the model containing the relationship between involvement in the online Autistic community and Autistic identity equally. Rather than testing whether communication preference has a direct impact on the variables, the SEM tested whether the relationship existed in the same way in both groups. Findings across the regression model and SEM all indicate that communication preference does not impact the relationship between involvement in the online Autistic community and Autistic identity.

The non-significant impact of communication preference on involvement and Autistic identity remained non-significant after variance in involvement and identity accounted for self-determination was removed from the relationship in the SEM. This 
finding provides further support for the lack of influence of communication preference on the relationship. Self-determination may account for a portion of the covariance of involvement and Autistic identity. Thus, findings eliminate the possibility of nonsignificance being related to self-determination variance by including selfdetermination in the SEM and finding positive relationships in both groups (prefers online communication and does not prefer online communication) as well as an overall model fit between the two groups. While other variables may be important for understanding the impact of communication preference on involvement in the online Autistic community and Autistic identity (e.g., verbal communication skills, computer and Internet skills, well-being, or social support), the SEM provided greater support for the nonsignificant finding beyond the regression model.

The non-significant finding of the impact of communication preference on the relationship between involvement in the online Autistic community and Autistic identity aligns with previous research on the development of personal and community identity within the online Autistic community that does not specifically account for communication preference (Bagatell, 2007, 2010; Brownlow \& O’Dell, 2006; Burke, Kraut, \& Williams, 2010; Davidson, 2008; Kidney, 2012; Silverman, 2008; Yergeau, 2010). Qualitative research and personal accounts discuss the benefits of online communication for autistic adults over face-to-face or verbal communication (Blume, 1997b; Burke et al., 2010; Davidson, 2008), yet no study has directly investigates whether preference moderates positive outcomes of involvement in the online Autistic community. 
Non-significant findings of the impact of communication preference on the relationship between involvement in the online Autistic community and Autistic identity suggest the benefits to all autistic adults regardless of communication abilities and preferences. While a person who prefers online communication may use the Internet as a primary source of relationships or community, those who prefer other types of communication equally develop a sense of identity when involved in the community online. Findings may be interpreted as contrary to previous research that indicates that a preference for online communication is seen as a risk factor for negative outcomes, such loneliness and depression (Caplan, 2003). Rather, communication preference has no impact when considering the positive outcome of Autistic identity.

As first-hand accounts of the online Autistic community anecdotally show, there are significant benefits of involvement in the online Autistic community, including making connections with like individuals to gain greater understanding of oneself. The finding that communication preference does not influence the positive impact of involvement in the community shows that all autistic individuals-regardless of preference-may benefit from inclusion. Communication preference may be due to differences or discomfort with face-to-face communication. This finding may suggest that regardless of face-to-face communication differences, autistic individuals may benefit from connecting with others online.

Self-Determination. In addition to examining the impact of communication preference on the relationship between involvement in the online Autistic community and Autistic identity, I also examined communication preferences in analyses containing 
involvement and self-determination. The regression model containing communication preference as a moderator in the relationship between involvement in the online Autistic community and self-determination, communication preference is not a significant predictor or interaction term, nor is communication preference significant in the regression model containing population type (autistic or non-autistic). Therefore, this analysis supports that higher levels of involvement in the online Autistic community are related to higher self-determination in all autistic adults, regardless of communication preference.

Additionally, the SEM comparison model containing involvement in the online Autistic community and self-determination in autistic adults who prefer and do not prefer online communication shows overall measurement invariance across models, suggesting the model fits the data equally well in all autistic adults regardless of communication preference. However, the model in autistic adults who do not prefer online communication contains a nonsignificant relationship between involvement in the online Autistic community and self-determination, but a significant relationship between the same variables in the model comprised of individuals who prefer online communication. These findings suggest that the relationship between involvement in the online Autistic community and self-determination is not present in autistic adults who do not prefer online communication within the overall model.

Specifically, the regression models containing self-determination support that communication preference does not influence the relationship between involvement in the online community and self-determination; as involvement in the online community 
increases, self-determination increases across all individuals regardless of communication preference. However, the SEM provides support that the data in both groups of autistic adults fit the model containing the relationship between involvement in the online Autistic community and self-determination equally, but the relationship between involvement and self-determination is not significant in autistic adults that do not prefer online communication. Rather than testing whether communication preference has a direct impact on the variables, the SEM tests whether the relationship exists in the same way in both groups. Findings across the regression model and SEM indicate that while communication preference does not have a moderating impact on involvement and self-determination, the relationship is not present in the model comprised of autistic adults who do not prefer online communication. Additionally, post-hoc correlational analyses support no significant differences on the variables of involvement in the online Autistic community or self-determination in autistic adults who prefer and do not prefer online communication.

The different analyses that I conducted containing the variables of involvement in the online Autistic community, communication preference, and self-determination require closer examination due to the complexity of findings. A number of differences exist between the moderated regression models containing the same variables and the two-group SEM. First, the SEM accounts for variance from Autistic identity. That is, Autistic identity development may constitute an aspect of the relationship between involvement in the online Autistic identity and self-determination that communication preference does not impact. The study supports positive relationships between Autistic 
identity and both variables in the model, indicating shared variance. Since involvement is related to Autistic identity, and correlational analyses only support relationships between involvement and the relatedness dimension of self-determination, perhaps Autistic identity constitutes a significant portion of the relationship and belongingness aspects of involvement and self-determination in individuals who do not prefer online communication. Another difference between the regression models and the SEM is the unidirectionality of the regression models. Perhaps communication preference only impacts the relationship between involvement in the online Autistic community and self-determination when the model allows the variables to covary. Finally, while the SEM utilized mean imputation of missing data in order to bolster the sample size, while the regression model utilized listwise deletion for missing data. Perhaps the inclusion of 29 additional cases in the SEM or the bias in covariance due to mean imputation creates the SEM findings. The two-group comparison SEM looks at the entire model and compares model fit across the two groups. Therefore, one cannot consider communication preference a moderating variable, but a grouping variable by which complex sets of variables and relationships may be compared.

The findings suggest that autistic adults who prefer online communication preference are different than those who do not prefer online communication in whether the relationship between involvement in the online Autistic community and self-determination exists in the model also containing Autistic identity. However, communication preference does not directly impact the relationship between involvement in the online Autistic community and self-determination. Perhaps the 
relationship between involvement and self-determination does not exist in autistic adults who do not prefer online communication because an increase in involvement and self-determination is not present unless Autistic identity increases. Autistic identity increases with higher involvement in the community for all autistic adults, but perhaps for individuals who have a preference for face-to-face or other types of communication an increase in self-determination only occurs as Autistic identity increases.

Previous literature characterizes communication in autistic adults to be difficult in face-to-face interactions, thus making the Internet a preferred mode of communication (Davidson, 2008). While communication preference in the current study has no impact on whether involvement in the online community is related to Autistic identity or self-determination, involvement is not related to self-determination in autistic adults who do not prefer online communication. This suggests that communication preference is relevant for self-determination in all autistic individuals. Research has shown that computer and Internet technologies may make learning and communication easier in education and therapies for autistic individuals (Rynkiewicz, 2013; Wentz, Nyden, \& Krevers, 2012). Current study findings suggest that computer and Internet technologies may result in Autistic identity development and selfdetermination. Further, while not important when developing Autistic identity, communication preference should be considered for developing self-determination in addition to identity. Specifically, education, training, or therapies may be more effective if they take into consideration differences in communication preference and how involvement in communities may be more beneficial to individuals' self-determination 
and identity. In autistic individuals who do not prefer online communication, it is possible that either Autistic identity or self-determination is fostered offline at different paces within the separate contexts. Such individuals may have higher identity or selfdetermination due to offline influences, leading to the variables not covarying within the current study.

Additionally, communication preference as a defining variable of the separate groups is potentially problematic in interpreting findings. As online communication and Internet use increases amongst all populations, perhaps the difference between online and face-to-face communication is narrowing, with opportunities to communicate both ways when either is optimal for the context. The actual impact of communication preference may be due to access to technology and opportunities to make choices in communication style rather than differences or difficulties with face-to-face communication. Additionally, as technology progresses, many devices allow for more options for communication, such as communication options available in many cell phones that range from text, instant message, video chat, to audio-only. A deeper understanding of individuals' communication preference is necessary. Further, no information is available in the current study to determine the quality and strength of relationships and community offline, which would provide important information on the interpretation of communication preference.

Further research with larger samples should examine the complexity of these relationships due to the data suggesting a slightly different impact of communication preference on Autistic identity and self-determination. Since the relationship between 
involvement and self-determination is only significant in autistic adults who prefer online communication, findings support increased attention to and education in Internet and online community access for autistic individuals who prefer online communication.

Additionally, no significant difference in self-determination exists between autistic adults who prefer online communication or who do not prefer online communication. However, in all individuals (autistic and non-autistic), individuals who do not prefer online communication had higher self-determination than individuals who prefer online communication. This finding aligns distally with previous research that indicates that in the general population, online communication preference is a risk factor for negative outcomes such as loneliness and depression (Caplan, 2003). Perhaps this finding is due to an increase of skill or comfort in face-to-face communication resulting in a greater perceived ability to be autonomous and competent in controlling life. Alternatively, internalized views of the superiority of face-to-face communication may result in autistic adults feeling less self-determined.

Overall, findings support the importance of considering communication preference when examining self-determination. The current study measured preference for online communication rather than communication skills or abilities. Therefore this study may be tapping into individuals' insecurities with other types of communication. Lacking confidence in other forms of communication may indicate lower perceived ability to have control over life or lower self-determination. Further, the study considered self-determination a broad concept that impacts all aspects of a person's 
life. However, perhaps involvement in the online Autistic community only relates to selfdetermination within the specific context of the Internet. Context-specific selfdetermination may differ in its relationship to involvement in the online community. Additionally, Ryan and Deci's (2000) theory of self-determination that the current study applies is designed to apply to all individuals. However, autistic individuals likely experience self-determination through a unique lens of marginalization and oppression. Perhaps the application of a disability or autistic-specific theory of self-determination would provide more valid results.

Due to the communication benefits, many autistic individuals have anecdotally expressed a preference for online or text-based communication over face-to-face interactions (Blume, 1997b; Burke et al., 2010; Davidson, 2008). Despite empirical evidence supporting negative outcomes for individuals who prefer online communication, the current study aims to examine the online community through a perspective that unique communication styles are not innately negative, but may promote positive outcomes within supportive contexts online. However, the findings indicated no direct impact of communication preference besides the finding that suggested involvement in the online Autistic community and self-determination are not related in autistic adults who do not prefer online communication.

\section{The Relationships among Involvement in the Online Autistic Community, Autistic} Identity, and Self-Determination 
While the SEM provides the model differences between autistic adults who prefer and do not prefer online communication, the analysis also offers support for the relationships among variables within the limitations of the sample and exploratory nature of the SEM. Correlational relationships and regression models provide information on the positive relationships between involvement in the online Autistic community and Autistic identity and involvement in the online community and selfdetermination. However, the associations shown in the regression model do not include communication preference. The regression models also include a variable to separately assess the relationships of self-determination and Autistic identity with the variable of involvement in the online Autistic community and how communication preference mediates the relationships. SEM findings support the relationships among all three variables in autistic adults, with positive covariances between involvement and Autistic identity, involvement and self-determination, and Autistic identity and selfdetermination. This finding uniquely supports the complexity of relationships in the online Autistic community, suggesting the variables are inter-related and dynamic rather than unidimensional and distinct from one another.

Involvement in the online Autistic community also was associated with selfdetermination in the SEM, which could be interpreted as involvement in the online Autistic community contributing to an increase in a person's self-determination as well as self-determination contributing to a person's skills, resources, and desire to become involved in the online Autistic community .Due to the cross sectional nature of the 
study, the directionality of the relationships in unknown in both the regression and SEM models. Future research should evaluate the directionality of these associations.

Beyond immediate implications of the relationships in the data, findings align with previous research that a positive minority identity may lead to positive psychological outcomes for an individual, such as a sense of pride and well-being (Berkman, Glass, Seeman, \& Brisette, 2000; Gill 1997). Autistic identity may provide the confidence, tools, and connections with others that may relate to autonomy, competence, and relatedness, the components of self-determination. Specifically, the dimension of relatedness is conceptually aligned with the Autistic identity dimensions of integration into the Autistic community and integration into society. Autistic identity reflects an Autistic individual's pride in being Autistic. Feelings of pride and confidence likely align with the self-determination dimension of autonomy.

Autistic identity and self-determination also co-vary with involvement in the online Autistic community in the model. Findings align with previous research that indicates involvement in communities is related to positive outcomes. In autistic individuals, involvement in online communities is related to closer relationships (Mazurek, 2013), pride and a strong group identity (Bascom, 2012; Ward \& Meyer, 1999). The Autistic community experiences marginalization and oppression, which autistic adults may seek community to gain support and solidarity, while also developing Autistic identity and self-determination. In autistic adults who do not prefer online communication, the relationship between involvement in the online Autistic community and self-determination is nonsignificant. The nonsignificant finding, as previously 
discussed, may be due to individuals with a preference for online communication having a greater need for online interactions to increase self-determination while individuals without a preference for online communication may seek community and tools for increasing self-determination in other contexts. However, the relationships between Autistic identity and involvement in the online Autistic community and Autistic identity and self-determination remained significant in the model, still promoting the positive aspects of the online Autistic community even for autistic adults who do not prefer online communication.

\section{Impact of Autism-Quotient Score Cut-Off on Findings}

The inclusion criteria for the autistic sample only asked if participants considered themselves to be on the autistic spectrum regardless of whether they have an autism diagnosis or scored above 26 or 32 on the AQ. The AASPIRE team determined this inclusion criteria based on the under-diagnosis of autism (Brugha et al., 2011; Jordan, 2010) and because the $A Q$ have been shown to have biases (Kim et al., 2011). Therefore, the sample of autistic adults in the current study may be more representative of autistic adults than if the sample excluded individuals based on diagnosis of AQ score. However, I conducted sensitivity analyses to explore whether considering the AQ cut-off scores of 26 and 32 impacted study findings.

Despite limitations of conducting additional post-hoc analyses in the same small cross-sectional sample, most of the sensitivity analyses suggested results that were similar in statistical significance to the corresponding analysis in the full autistic sample. 
Analyses suggested significant relationships between involvement in the online Autistic community and Autistic identity in both subsamples of autistic adults with AQ cut-off scores of 26 and 32 . The relationship between involvement in the online Autistic community and self-determination was also significant in both subsamples of autistic adults. Communication preference did not moderate the relationships between involvement in the online Autistic community and Autistic identity or involvement and self-determination, similarly to the statistical significance in the full sample. Attending to these limitations, the findings suggest that an $A Q$ score does not impact the relationship between involvement in the online Autistic community and Autistic identity or selfdetermination. The shortcomings of the AQ score as an unbiased measure of autistic traits (Kim et al., 2011) may be responsible for similar findings across subsets of the sample. Alternatively, the online Autistic community may be a supportive and positive environment that is related to greater Autistic identity and self-determination regardless of whether a person has more autistic traits. That is, those identifying as Autistic may find support and self-determination regardless of how similar they are to the stereotypical traits of autism. Perhaps this is exemplified in "autistic cousins," or individuals who have social or communication differences but are not be diagnosed with autism (Sinclair, 2005). Non-autistic individuals may still find similarities with others in the community, and may build relationships and gain knowledge from the online Autistic community. Regardless of AQ score, individuals may find strong connections and positive outcomes when involved in the online Autistic community. 
However, the sensitivity analysis in the SEM final model, the fit indices suggested a poor model fit, unlike in the full sample. Due to the sample requirements of SEM, performing an analysis with fewer individuals than the current sample further limits the power and the ability to find good model fit. The difference between the full sample and the subsample comparison should be interpreted with caution. Without further research and statistical comparisons among the subsamples, findings are unclear about where model changes may be appropriate and therefore how the poor model fit may be interpreted.

\section{Strengths and Limitations}

The study has many strengths, including that it concerns the under-researched population of autistic adults. Further, it involves a large sample of a homogenous and unresearched segment of autistic adults, those seeking community online, using efficient methods to gather data in the easiest possible manner for the participant. The community-engaged approach implemented in the current study creates strengths as well. The community-engaged research process allowed the study to examine research questions that the community provided input on during the development of the study, creating greater relevance and merit for autistic adults. In the overarching larger study, AASPIRE aimed to answer questions about the online Autistic community and empirically support the positive impact the community seems to have on involved individuals. The team collaborated to create the protocol, materials, and measures, so 
unique perspectives from researchers, practitioners, family members, service care providers, and autistic adults were all taken into account to improve them. The processes in this study involved communication and feedback from community partners about the purpose, aims, findings, and interpretations so that the study findings and implications would be strengths-based and focused on improving the lives of autistic individuals.

In addition to engaging community partners, this study was the first to utilize, adapt, and examine Gill's Disability Identity Scale (1997a) for autistic adults. The study supported the scale being a plausible representation of Autistic identity. An EFA revealed a number of items that did not fit the subscales well, and due to the community-engaged processes of the study, community partners added additional support for removal of those items. The evaluation of the Autistic Identity Scale was an exploratory, data-driven aspect of this study that may contribute to future research. Future research may utilize the adapted scale in order to determine whether the scale is valid in other autistic samples. Research may also examine the scale and subscales with confirmatory factor analyses based on the assignment of items to subscales in the current study. Lastly, researchers may use the scale in different contexts or with different predictors or outcomes to further examine Autistic identity. The current study findings provide psychometric information on the scale to support its use for additional research.

The study also has several important limitations that must be considered in interpreting study findings. First, limitations to internal validity include that the study 
relies on cross-sectional data collected from a purposeful sample, resulting in an inability to draw causal or comparative inferences from the data. Specifically, the data cannot infer that involvement in the online Autistic community causes positive outcomes such as Autistic identity and self-determination. Also, the study excluded related constructs that may impact the variables such as social support and empowerment. Measuring such variables could shed light on the complicated relationships among the constructs of this study, since perhaps additional variables moderate the relationships between involvement in the online Autistic community and other positive outcomes. For example, social support is assumed in involvement in the online Autistic community, but if social support was measured it may account for some of the variance between involvement and self-determination.

The study supported associations between involvement in the online Autistic community and Autistic identity as well as self-determination. The unidirectional hypotheses of involvement in the online Autistic community predicting Autistic identity and involvement in the online Autistic community predicting self-determination were tested and supported. Additionally, the SEM tested and supported bidirectional relationships among the variables. However, the findings are based on cross-sectional associations, which cannot be inferred to support a unidirectional or bidirectional relationship. The study also does not allow for causal relationships among any of the variables. Future research should attend to the directionality of the associations supported in the current study with longitudinal designs. 
The study involved multiple complex analyses of the cross-sectional and relatively small sample. The number of comparisons and models conducted with the single sample increase the probability of type I error (Tabachnick \& Fidell, 1996). In particular, the small sample size and exploratory nature of the SEMs may have produced unreliable findings. The small sample may constrain the variance of key variables in analyses inappropriately for generalizing the findings to the population of autistic adults in the online Autistic community. These sample size considerations may be particularly relevant when comparing findings from the planned analyses to the sensitivity analyses conducted with only subsets of the autistic sample that scored 26 or higher or 32 or higher on the AQ. Analyzing a smaller sample decreased the power of the analyses, which may be responsible for non-significant findings in the subsamples. A larger sample size may lead to greater precision in findings, which is important in studies such as the current study in which parameters have not been previously studied. A larger sample may create an opportunity to perform a confirmatory analysis on a segment of the sample after I conducted exploratory analyses.

While limitations of the sample size exist, the limitations are lessened by the descriptive analysis findings in the sample that showed normal distributions of the variables after data cleaning. Without future research, the generalizability of findings is unknown, so confirming the findings of this study, particularly in larger populationbased samples, may be necessary for confident interpretations of the data.

Additionally, the study included participants in two dichotomous samples of autistic adults and non-autistic adults. The study included non-autistic adults who 
identify as individuals with disabilities in the non-autistic adult sample for the current study. However, perhaps individuals with disabilities experience online communities and self-determination more similarly to autistic adults than non-autistic adults, due to shared experiences of marginalization. However, the study did not consider disabilities in exclusion from the autistic sample; therefore the sample biases of individuals with disabilities are present in both samples. Controlling for or excluding participants that identify as individuals with disabilities may result in significant differences between autistic and non-autistic samples.

The use of mean imputation for the structural equation modeling poses an additional limitation. The analysis required imputation of missing data in order to have an adequate sample size, as SEM does not allow for listwise deletion, and a number of cases included missing data. While the literature considers mean imputation an appropriate method of missing data imputation, mean imputation does not take into consideration participant-level patterns of scores across other variables (Carter, 2006), and results in biased variance by imputing the mean score with no variance (Kline, 1998). Given the relatively small data set for SEM and the low percentage of cases with missing data (16.56\%), I utilized mean imputation, though a more intensive imputation method for missing data may allow for less biased variance, which may result in more accurate findings.

Threats to construct validity also exist. Due to the collecting data from a single sample, it is impossible to retest the model fit and validity to ensure they were not methodological artifacts or are unique to the sample tested. Additionally, results of the 
Involvement in the online Autistic community PCA and the Autistic Identity Scale EFA may be distorted due to selective sampling with a unique factor of autistic adults seeking community online. However, the measurement development contributions to the literature serve as important progress in understanding the concepts of involvement in the online community and Autistic identity. A multi-dimensional measure of involvement in the online community was developed in the study, and can be applied to other studies that examine outcomes or positive relationships with involvement in online communities, including further research with the online Autistic community. Other threats to construct validity exist. The construct of involvement in the online Autistic community contains a dimension of frequency of involvement that is measured by a categorical measurement assumed to be continuous for this study, which may impact the composite variable. Little research examines the involvement in the online Autistic community, so no a priori assumptions were made about the dimension weighing composition of this variable, other than inclusion of chosen domains. Using mostly data-driven weights for the composition of this variable may have resulted in a theoretically unsound measure overly reliant on item variance. However, as the study is the first of its kind to examine the multi-dimensional concept, using a data-driven approach was a logical first step. I recommend future research on the theoretical and data-driven validity of the measure.

Additionally, one should take into account the construct validity of the selfdetermination measure. As the study conducted secondary data analysis, a selfdetermination measure was constructed from subscales of the measures utilized for the 
AASPIRE Internet Use, Community, and Well-Being Survey, and did not utilize preexisting, validated measures of self-determination. Therefore, it may be more appropriate to consider the measured construct in the current study one indicator of self-determination. However, the available data from the Psychological Well-Being Scales and SCl-2 compromised a theoretically appropriate measure with good internal consistency reliability and good face validity. Though I did not utilize a previously used self-determination measure, I closely approximated the subscales of the Ryan and Deci (2002) self-determination theory. Since the measurement of self-determination varies widely in the literature, such inconsistencies with pre-existing measures were acceptable. Further, Ryan and Deci's self-determination theory excludes a number of dimensions that are considered in other theories and measures of self-determination, particularly for individuals with disabilities, including self-regulation, empowerment, and self-realization (Wehmeyer, 1996; 1997; 1999; 2001). Inclusion of these dimensions may increase the validity of the self-determination measure in the current study. However, Ryan and Deci's self-determination theory encompasses three dimensions that this study explores as a whole construct as well as separate, unique outcomes that may provide evidence for the positive outcomes of autonomy, environmental mastery, and relatedness in the online Autistic community. Findings should be interpreted cautiously, with knowledge that other theories contain different and additional dimensions of the construct of self-determination. On the other hand, Ryan and Deci's (2000) theory also provides strength in analysis involving the comparison between autistic and non-autistic individuals, because it omits constructs that the community partners of AASPIRE 
considered irrelevant and difficult to measure, including self-realization. Abstract concepts were intentionally removed from the survey to reduce participant frustration and increase validity of responses, with questions about autonomy and environmental mastery easier conceptualize. Therefore the measure of self-determination in autistic and non-autistic adults may be more reliable across the populations.

Limitations to the external validity exist in the generalizability of results from the measurement development analyses. The sample was a convenience sample of all interested autistic and non-autistic adults who were contacted to conduct the study online. This sample may have been limited by sample bias. Autistic and non-autistic individuals were recruited via websites, blogs, and listservs that primarily function for autistic individuals or individuals with disabilities. It is likely that individuals with stronger connection to the online Autistic community were more willing to participate, and individuals who do not have connection to the online Autistic community were excluded from the sample. Further, it is unlikely that non-autistic participants found out about the study via an online community that they strongly identify with or that would impact their self-determination. The sample bias also impacted the measurement of communication preference. Participants' decision to be involved in online communities may be a result of a preference to communicate online. Therefore recruitment and participation of individuals may have been biased towards those with online communication preference, which was confirmed in the whole sample characteristics with $60 \%$ of the sample not preferring online communication. While I weighted the variable to account for unequal communication preference in the moderated regression 
analysis, perhaps a more evenly distributed sample would impact study findings. While the sample has limitations and biases, the matching process AASPIRE utilized for recruiting non-autistic adults strengthens the comparisons made between samples. Non-autistic adults were recruited based on matching the autistic sample on demographic characteristics. This allowed the samples to be similar in demographics and more precisely specify that differences are due to being in the different communities of autistic or non-autistic individuals. Future research should utilize comparison groups that have similarly strong communities, and recruit participants from a wider population with varied communication preferences and ties to communities. Additionally, future research should include communities that are not online, including the Autistic community outside the Internet. Such research may shed light on how the online Autistic community is uniquely or similarly supportive as other communities.

The sample demographics were compared between autistic and non-autistic individuals and no significant differences exist. However, the demographics show a relatively uniform sample of white, highly educated adults. Such individuals may have a unique experience of involvement in online communities that limits how comparable findings may be in the sample as compared to other individuals not in the study sample. Research shows that Internet users encompasses a wide and diverse population of people, with $82 \%$ of all US adults using the Internet with incomes ranging from less than $\$ 30,000$ to over $\$ 75,000$ (Zickuhr, 2012). While Internet use is less in lower incomes, the current study sample is lower income and may over-represent those individuals. Pew 
Internet Research (2012) also shows that 58\% of individuals with no high school diploma use the Internet, with increasing percentages of Internet use in groups of Americans with higher educational attainment. The current study sample contains mostly highly educated individuals, which may not reflect the actual population of Internet users and have an impact on findings. For example, perhaps higher education impacts the flexibility and lack of preference in communication. Additionally, while White, NonHispanic individuals have the highest percentage of Internet users (84\%), $77 \%$ of Black, Non-Hispanic individuals use the Internet and $75 \%$ of Hispanic individuals use the Internet (Zickuhr). The mostly white sample of the current study also does not reflect the population of Internet users. Autism impacts all races/ethnicities and income levels, so the sample demographics should be closer to general population statistics. The large differences between the Pew Internet Research demographics and the current study demographics indicate the uniqueness of the current study sample. Future studies should seek a larger and more diverse sample that more closely mirrors Internet use across different race/ethnicities and education levels. The study findings may be more appropriately generalizable to only others with lower income, higher education, and white race.

In addition to the potential sample biases related to communication preference, the variable of communication preference was dichotomized into prefers online communication and does not prefer online communication. Those who do not prefer online communication may prefer face-to-face communication, other types of communication, or may not have any preference. The study was limited in not exploring 
the differences among those with various communication preferences. Future research may explore how specific preferences impact individuals' involvement in online communities.

Due to the specified population, findings may not be generalizable beyond autistic and non-autistic adults who seek out community online. However, as the concepts of involvement in community apply across all types of communities, a measurement should easily adapt for use in different communities. Additionally, while Autistic identity is specific to the population of interest, research has shown a similar identity development in other marginalized communities, such as the gay and lesbian community (Cramer \& Gilson, 1999). Therefore, some of the study findings may apply to similar communities, particularly the disability community.

Beyond limitations of the validity of this study, threat exists to the Autistic community in the potential misinterpretation or misuse of the data. This threat is particularly relevant in the utilization of a cross-cultural group comparison, including for the finding that compares self-determination in autistic adults to non-autistic adults. One must interpret such a comparison cautiously due to the existing differences between the groups, including the differences between the online Autistic community and the communities of reference for non-autistic participants, as well as the different meanings of communication preference and self-determination between individuals in the two comparison groups. These differences may be due to recruitment strategies that targeted Autistic communities but not specific communities for non-autistic individuals, as well as the historical oppression and challenges faced by autistic 
individuals that impact communication and self-determination. Further, turmoil in the community that resulted in negative feelings and disjointed relationships among members of the online Autistic community may prove to create a significant difference between autistic and non-autistic's involvement in online communities and selfdetermination. Specifically, many questions in the survey provided examples of websites, listservs, and blogs that are part of the online Autistic community. Some of the examples listed throughout the survey directly identify the websites that were combative at the time of data collection. Answering questions about the online Autistic community while being reminded of the disharmony within it may have greatly impacted responses. One should consider this historical context when interpreting findings from the autistic sample, since such turmoil did not necessarily impact nonAutistic communities. However, utilizing a comparison group of non-autistic adults provides stronger evidence towards understanding the unique communication preferences and the unique impact of involvement in the online Autistic community as related to positive outcomes for autistic adults. No study examines whether positive outcomes of online involvement are greater for autistic adults than non-autistic adults.

\section{Implications}

There exist important implications of the overarching aims and specific findings of this study. The study and its findings may potentially impact theory, research, and action. 
Theory implications. The current study contributes findings to theories of Autistic identity and self-determination. Specifically, application of these theories to autistic individuals shows the utility of these theories within the context of the Autistic community. Gill's (1997) theory of disability identity is utilized for the current study and examined in terms of the Autistic community. Despite limitations to the small sample and exploratory analyses, findings suggest that Gill's theory is applicable to the Autistic community, and that associations between disability identity and positive outcomes such as self-determination and higher social support (Lee, Lee, Rhee, Shin, \& Lim, 2008) may also apply to autistic individuals. Self-determination in autistic adults has not been quantitatively examined prior to the current study. The current study therefore contributes initial exploratory findings about the application of self-determination theory to this specific population. Ryan and Deci's (2000) self-determination theory can be applied in autistic populations based on findings of the current study.

The finding that autistic adults who do not prefer online communication do not have a positive relationship between involvement in the online Autistic community and self-determination also has implications, despite limitations to the dichotomous variable utilized in the study (prefers online communication versus does not prefer online communication, including no preference and preference for face-to-face communication). While communication preference does not moderate the relationship, these variables have a different relationship in autistic adults who prefer online communication. It is important for future research to identify what impact communication preference does have on the relationship between involvement in the 
online community and self-determination, in order to determine how the two groups differ. Similarly, individuals who prefer online communication have significantly less selfdetermination than individuals who do not prefer online communication. Such finding indicates the need to research the communication preference and what other characteristics are associated with preference to communicate online, such as skills to make self-determined choices offline.

Research implications. Research implications also exist from the study. Despite the limitations of conducting analyses with a single small sample in the current study, the measurement development analyses with Gill's Disability Identity Scale (Gill, 1997b) contribute to the literature where no studies have investigated the scale. Similarly, the measure of involvement in the online community was adopted for the current study, contributing initial measurement development to the literature. This study is an important first step in improving the measures and creating useful tools for measuring Autistic identity or disability identity.

While considering the limitations of the study, the significant finding that selfdetermination in non-autistic adults is higher than self-determination in autistic adults still has implications for further research in the Autistic community. Further studies should examine what other indicators may contribute to the differences between autistic and non-autistic adults in self-determination, or whether the difference may have contributed to measurements in the current study.

Additionally, the exploratory SEM analyses in the current study provide a first step for developing a theoretically-based model exploring involvement in the online 
Autistic community, Autistic identity, and self-determination. Further research can refine the model and explore the fit in larger and different samples, such as a sample including more diverse racial/ethnic groups in the online Autistic community.

Action implications. While the study findings have more direct impact on research and theory, this study may also somewhat contribute to social action that may positively impact the online Autistic community. I discussed limitations of the study earlier, including the cross-sectional survey methodology which does not allow causal inferences of outcomes based on involvement in the online Autistic community. Additionally, no variables are included in the current study that could illuminate how autistic adults were introduced to computers, Internet use, or online communities. However, study findings may lead more broadly to different ways of viewing the Autistic community and the importance of education and training in computer and Internet use to lead to more distal outcomes that may promote identity and self-determination.

By studying the unique population of the online Autistic community, the confusion and misinformation that has lead to stigmatization and marginalization in autistic adults may be reduced (Boundy, 2008; Robertson, 2010). Empirical evidence of the positive relationship between involvement in the Autistic community and selfdetermination may shed positive light on a community that has often been studied in a negative, deficits-based manner (Silverman, 2008). By focusing on positive outcomes of involvement in the online Autistic community, this study can empirically negate inaccurate stereotypes about what it means for an autistic individual to live a meaningful life with peer support and community, and inform mechanisms that foster 
such positive outcomes for this under-researched population through strengths-based research. As Internet use continues to increase over time, research such as the current study can promote and begin to understand the positive utility of such a powerful global tool in promoting identity and self-determination. Also, understanding the impact of new media on Autistic self-advocacy contributes to the community's call for research that is relevant to the Autistic community and the need for research that has a positive impact on the community (Robertson, 2010).

Examining the relationships between involvement in the online community and Autistic identity and self-determination provided preliminary evidence for relationships between positive outcomes such as self-determination and involvement in the community by increasing social relationships and support, information about themselves and the community, and opportunities for self-reflection, sharing, and advocacy, particularly for autistic adults who prefer online communication. Research shows that self-determination has a powerful positive impact on individuals with disabilities, including a higher quality of life and life satisfaction (Cagle, 2006; Lachapelle et al., 2005; Nota, Ferrari, Soresi, \& Wehmeyer, 2007) and employment outcomes (Lachapelle et al., 2005; Shogren et al, 2008; Wehmeyer \& Schwartz, 1998). Therefore, the findings of the current exploratory study may indirectly promote education and research to lead to the positive outcomes related to self-determination. Implication for specifically understanding the impact of communication preferences exist as well. Communication preference does not have an impact on the relationship between involvement in the online Autistic community and Autistic identity in the current study. 
Therefore, involvement in the online Autistic community is positively related to Autistic identity in all autistic adults, showing the widespread benefits derived from the community. The initial exploratory finding of a lack of a difference between communication preferences supports the need to promote policy and education that allows all autistic adults to use computer and Internet technologies, particularly for interventions, classrooms, or therapies.

Current study findings provide initial findings that illuminate the social and communication differences among autistic individuals, a group that is often unfairly categorized by perceived functionality based on neurotypical norms rather than personal preference or contextual, individualized ability (Davidson, 2008). Such knowledge may more distally inform policy and education designed for autistic individuals who prefer online communication over face-to-face or other methods of communication. While the current study findings do not suggest specific ways to improve policy or education, these exploratory findings suggest that involvement online may be beneficial to autistic adults and therefore should be more accessible and flexible to different communication preferences. Research suggests that computer-based interventions may be effective for building communication skills for autistic children (Ramdoss et al., 2010). However, the impact of computer-based interventions on selfdetermination is not widely known or researched, particularly in autistic adults. Additionally, no research has been conducted on the impact of training to use Internet communities. Future research may explore specifically how computer and Internet education may impact positive outcomes. The last finding that has implications for social 
advocacy and change is the significant finding that self-determination in non-autistic adults is higher than self-determination in autistic adults. Further research should determine why the difference in self-determination exists in the current small and crosssectional sample and how self-determination may be increased, and findings should be applied to social action to promote self-determination to increase equality between autistic and non-autistic adults. While this exploratory study was a cross-sectional of a small sample, findings may potentially impact how individuals perceive autistic individuals and the online Autistic community regarding community, social interactions, and communication preferences. Further, findings may impact policy and education for autistic individuals to increase Internet use and involvement in online communities. 
Table 1. Self-determination theory constructs and measures in current study

\begin{tabular}{|c|c|c|c|}
\hline Construct & Source & Description & Measure \\
\hline Self-Determination & $\begin{array}{l}\text { Ryan and } \\
\text { Deci (2000) }\end{array}$ & $\begin{array}{l}\text { Psychological needs must develop within } \\
\text { an individual including autonomy, } \\
\text { competence, and relatedness. If the } \\
\text { environment an individual develops within } \\
\text { is supportive, the presence of these three } \\
\text { psychological needs will result in a self- } \\
\text { determined individual. }\end{array}$ & $\begin{array}{l}\text { Composite score of } \\
\text { "Autonomy," } \\
\text { "Environmental Mastery," } \\
\text { and "Shared Emotional } \\
\text { Connection" scales }\end{array}$ \\
\hline Autonomy & $\begin{array}{c}\text { Ryan and } \\
\text { Deci (2000) }\end{array}$ & $\begin{array}{l}\text { A sense of volition, willingness, and } \\
\text { congruence, wherein individuals decide on } \\
\text { the behavior they engage in. }\end{array}$ & $\begin{array}{l}\text { "Autonomy" scale of the } \\
\text { Ryff Scales of Psychological } \\
\text { Well-Being (Ryff, 1989; 9- } \\
\text { item version) }\end{array}$ \\
\hline Competence & $\begin{array}{c}\text { Deci \& } \\
\text { Vansteenkiste } \\
\text { (2004) }\end{array}$ & $\begin{array}{l}\text { Individuals' abilities and effectiveness in } \\
\text { controlling aspects of their environments } \\
\text { and lives. }\end{array}$ & $\begin{array}{l}\text { "Environmental Mastery" } \\
\text { scale of the Ryff Scales of } \\
\text { Psychological Well-Being } \\
\text { (Ryff, 1989; 9-item version) }\end{array}$ \\
\hline Relatedness & $\begin{array}{c}\text { Deci \& } \\
\text { Vansteenkiste } \\
\text { (2004) }\end{array}$ & $\begin{array}{l}\text { The individual's need to interact with and } \\
\text { meaningfully connect to other people. }\end{array}$ & $\begin{array}{l}\text { "Shared Emotional } \\
\text { Connection" subscale of the } \\
\text { Sense of Community } \\
\text { Version } 2 \text { scale (Chavis et } \\
\text { al., 2008; } 6 \text { items) }\end{array}$ \\
\hline
\end{tabular}


Table 2. Participant Demographics

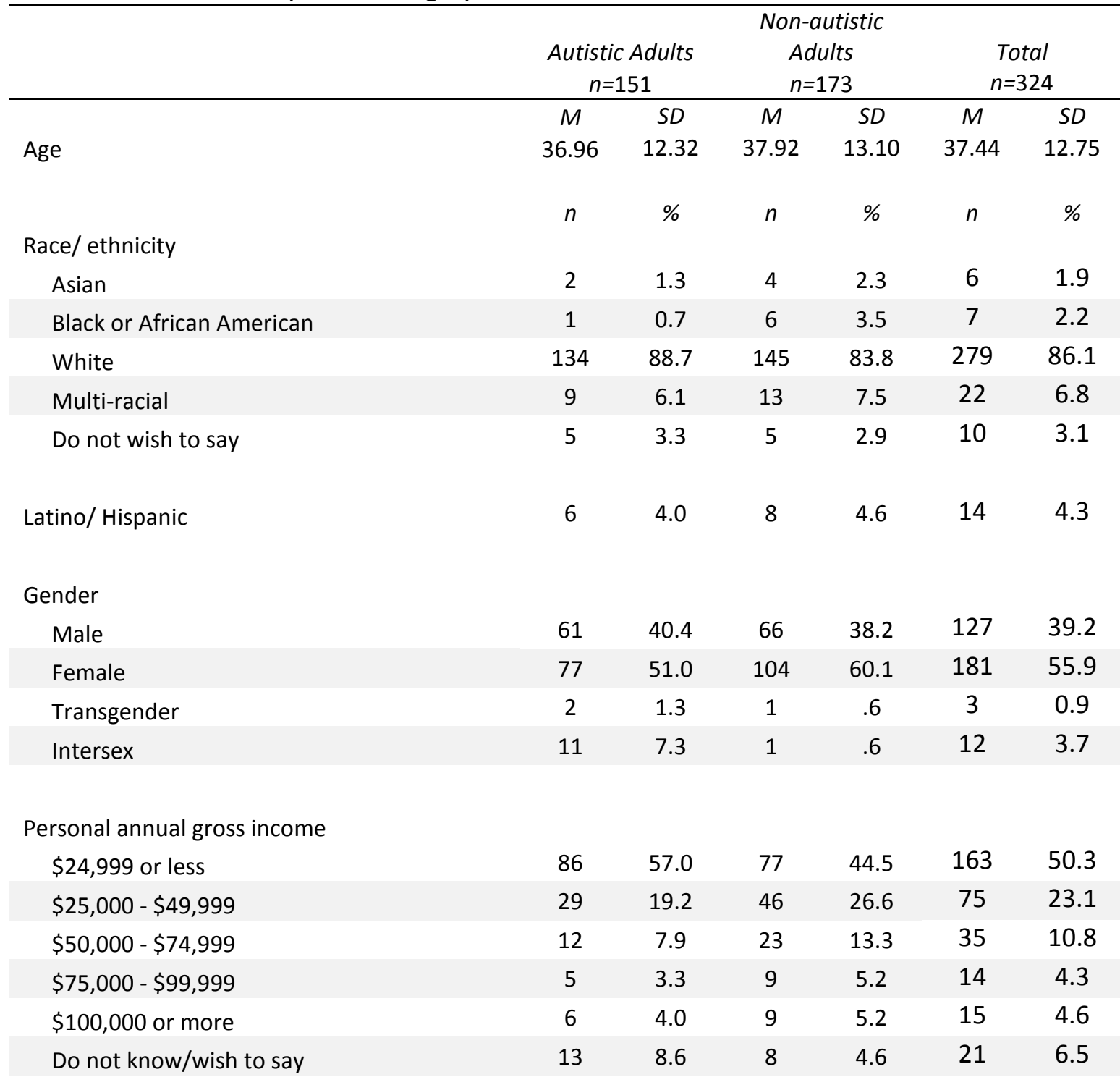

Education level

$\begin{array}{lcccccc}\text { Less than high school or equivalent } & 2 & 1.3 & 2 & 1.2 & 4 & 1.2 \\ \text { High school diploma or equivalent } & 10 & 6.6 & 3 & 1.7 & 13 & 4.0 \\ \text { GED (general equivalency diploma) } & 0 & 0 & 3 & 1.7 & 3 & 0.9 \\ \text { Post-secondary certificate/ technical degree } & 6 & 4.0 & 3 & 1.7 & 9 & 2.8 \\ \text { Some college or university courses } & 40 & 26.5 & 26 & 15.0 & 66 & 20.4 \\ \text { 2 year or Associate's degree or equivalent } & 12 & 7.9 & 6 & 3.5 & 18 & 5.6 \\ \text { Bachelor's degree or equivalent } & 33 & 21.9 & 48 & 27.7 & 81 & 25.0 \\ \text { Some graduate school } & 12 & 7.9 & 23 & 13.3 & 35 & 10.8 \\ \text { Master's degree or equivalent } & 26 & 17.2 & 35 & 20.2 & 61 & 18.8 \\ \text { Doctoral or professional doctorate } & 7 & 4.6 & 18 & 10.4 & 25 & 7.7\end{array}$




$\begin{array}{lcccccc}\text { Other } & 0 & 0 & 1 & 0.6 & 1 & 0.3 \\ \text { Do not know/wish to say } & 3 & 2.0 & 0 & 0 & 3 & 0.9\end{array}$

\begin{tabular}{lcccccc} 
Parent education level $^{1}$ & & & & & & \\
Less than high school or equivalent & 9 & 6.0 & 7 & 4.0 & 16 & 4.9 \\
Modified high school diploma & 2 & 1.3 & 3 & 1.7 & 5 & 1.5 \\
High school diploma or equivalent & 23 & 15.2 & 32 & 18.6 & 55 & 17.0 \\
GED (general equivalency diploma) & 0 & 0 & 1 & .6 & 1 & 0.3 \\
Post-secondary certificate/technical degree & 9 & 6.0 & 6 & 3.5 & 15 & 4.6 \\
Some college or university courses & 11 & 7.3 & 11 & 6.4 & 22 & 6.8 \\
2 year or Associate's degree or equivalent & 10 & 6.6 & 10 & 5.8 & 20 & 6.2 \\
Bachelor's degree or equivalent & 27 & 17.9 & 46 & 26.6 & 73 & 22.5 \\
Some graduate school & 8 & 5.3 & 2 & 1.2 & 10 & 3.1 \\
Master's degree or equivalent & 33 & 21.9 & 39 & 22.5 & 72 & 22.2 \\
Doctoral or professional doctorate & 14 & 9.3 & 16 & 9.2 & 30 & 9.3 \\
Other & 1 & 0.7 & 0 & 0 & 1 & 0.3 \\
Do not know or do not wish to say & 4 & 2.6 & 0 & 0 & 4 & 1.2 \\
& & & & & & \\
Employment status & & & & & & \\
Employed full-time at a regular job & 47 & 31.2 & 74 & 42.8 & 121 & 37.3 \\
Employed part-time at a regular job & 11 & 7.4 & 13 & 7.5 & 24 & 7.4 \\
Employed full-time via supported program & 1 & 0.7 & 0 & 0 & 1 & 0.3 \\
Volunteering & 9 & 6.0 & 28 & 16.2 & 37 & 11.4 \\
In school & 20 & 13.2 & 22 & 12.7 & 42 & 13.0 \\
In school and employed or volunteering & 19 & 12.6 & 18 & 10.4 & 37 & 11.4 \\
In a transition program & 2 & 1.4 & 1 & 0.6 & 3 & 0.9 \\
Seeking employment & 21 & 13.9 & 16 & 9.2 & 37 & 11.4 \\
Not seeking employment & 4 & 2.6 & 11 & 6.4 & 15 & 4.6 \\
Other & 16 & 10.6 & 4 & 2.3 & 20 & 6.2 \\
Do not know or wish to say & 0.7 & 3 & 1.7 & 4 & 1.2 \\
\hline
\end{tabular}

Note. ${ }^{1}$ Highest level of education that either parent or current legal guardian obtained. 
Table 3. Autistic traits and autism spectrum disorder diagnoses

Autism Diagnoses and Traits $^{1}$

$n=151$

$n$

$\%$

Autism status

Formally diagnosed

102

67.5

Not formally diagnosed

49

32.5

AQ score of 26 or higher ${ }^{2}$

Yes

138

13

91.4

No

8.6

AQ score of 32 or higher

Yes

123

81.5

No

27

17.9

Rett's disorder

1

0.7

PDD-NOS ${ }^{4}$

3

2.0

"On the autism spectrum"

2

1.3

Do not know/ wish to say

4

2.6

Notes. ${ }^{1}$ All participants included in current sample identified as autistic; "Yes" indicates a score of 26 or higher on the Autism Quotient (Baron-Cohen et al., 2001); "Response to the item: "Currently, I think that the autism spectrum diagnosis that fits me best is:"; ${ }^{4}$ Pervasive developmental disorder- not otherwise specified. 
Table 4. Involvement in the online community dimension descriptives and principal component weights

\begin{tabular}{|c|c|c|c|c|c|c|}
\hline \multirow[b]{3}{*}{ Years of involvement } & \multicolumn{2}{|c|}{$\begin{array}{l}\text { Autistic Adults } \\
\quad n=151\end{array}$} & \multirow{2}{*}{$\begin{array}{c}\text { Principal } \\
\text { Component } \\
\text { Weight } \\
\%\end{array}$} & \multicolumn{2}{|c|}{$\begin{array}{l}\text { Non-autistic } \\
\text { Adults } \\
n=173\end{array}$} & \multirow{2}{*}{$\begin{array}{c}\text { Principal } \\
\text { Component } \\
\text { Weight } \\
\%\end{array}$} \\
\hline & $M$ & $S D$ & & $M$ & $S D$ & \\
\hline & 5.25 & 3.66 & 8.41 & 9.93 & 5.85 & 12.35 \\
\hline Importance of involvement & 5.58 & 1.48 & 34.23 & 4.86 & 1.58 & 38.31 \\
\hline Sense of Community & 2.28 & 0.55 & 24.1 & 2.19 & 0.60 & 20.62 \\
\hline & $n$ & $\%$ & & $n$ & $\%$ & \\
\hline Frequency of involvement & & & 33.26 & & & 28.72 \\
\hline Never & 2 & 1.30 & & 2 & 1.16 & \\
\hline $\begin{array}{l}\text { Less than once a } \\
\text { month }\end{array}$ & 7 & 4.60 & & 2 & 1.16 & \\
\hline $\begin{array}{l}\text { At least once a month } \\
\text { but less than once a week }\end{array}$ & 10 & 6.60 & & 17 & 9.83 & \\
\hline $\begin{array}{l}\text { At least once a week } \\
\text { but less than once a day }\end{array}$ & 14 & 9.30 & & 22 & 12.72 & \\
\hline $\begin{array}{l}\text { Less than one hour } \\
\text { daily }\end{array}$ & 28 & 18.50 & & 29 & 16.76 & \\
\hline $1-3$ hours daily & 50 & 33.10 & & 67 & 38.73 & \\
\hline $3-6$ hours daily & 22 & 14.60 & & 25 & 14.45 & \\
\hline $\begin{array}{l}\text { More than } 6 \text { hours } \\
\text { daily }\end{array}$ & 18 & 11.90 & & 9 & 5.20 & \\
\hline
\end{tabular}


Table 5. Factor loadings of the Autistic Identity Scale Exploratory Factor Analysis

\begin{tabular}{|c|c|c|c|c|}
\hline & $\begin{array}{l}\text { Integration into } \\
\text { the Autistic } \\
\text { community }\end{array}$ & $\begin{array}{l}\text { Integration } \\
\text { into society }\end{array}$ & $\begin{array}{l}\text { Integration of } \\
\text { society and the } \\
\text { Autistic } \\
\text { community }\end{array}$ & $\begin{array}{l}\text { Integration of } \\
\text { feelings about } \\
\text { self/ presentation } \\
\text { of self }\end{array}$ \\
\hline $\begin{array}{l}\text { 5. I feel that people on the } \\
\text { autistic spectrum have made } \\
\text { major accomplishments and } \\
\text { advancements. }\end{array}$ & .46 & .42 & .54 & -.05 \\
\hline $\begin{array}{l}\text { 13. My future is tied to the } \\
\text { future of other people on the } \\
\text { autistic spectrum. }\end{array}$ & .48 & -.13 & .06 & .68 \\
\hline $\begin{array}{l}\text { 14. I feel good about people } \\
\text { on the autistic spectrum. }\end{array}$ & .86 & .09 & .36 & .14 \\
\hline $\begin{array}{l}\text { 17. I have a strong sense of } \\
\text { belonging to the Autistic } \\
\text { community. }\end{array}$ & .66 & -.13 & .41 & .57 \\
\hline $\begin{array}{l}\text { 18. I almost never tell people I } \\
\text { am on the autistic spectrum.* }\end{array}$ & .59 & .38 & .44 & .30 \\
\hline $\begin{array}{l}\text { 9. People on the autistic } \\
\text { spectrum contribute less to } \\
\text { society than others.* }\end{array}$ & .28 & .86 & .30 & -.04 \\
\hline $\begin{array}{l}\text { 10. Overall, I often feel that } \\
\text { people on the autistic } \\
\text { spectrum are not } \\
\text { worthwhile.* }\end{array}$ & -.02 & .85 & .27 & .12 \\
\hline $\begin{array}{l}\text { 1. I believe that because I am } \\
\text { a person on the autistic } \\
\text { spectrum, I have many } \\
\text { strengths. }\end{array}$ & .32 & .21 & .77 & .18 \\
\hline $\begin{array}{l}2 . \text { I often regret that I am a } \\
\text { person on the autistic } \\
\text { spectrum.* }\end{array}$ & .28 & .39 & .81 & .16 \\
\hline $\begin{array}{l}\text { 6. I am happy to be a person } \\
\text { on the autistic spectrum. }\end{array}$ & .39 & .16 & .85 & .24 \\
\hline $\begin{array}{l}\text { 8. Being a person on the } \\
\text { autistic spectrum is an } \\
\text { important reflection of who I } \\
\text { am. }\end{array}$ & .24 & .03 & .60 & .67 \\
\hline $\begin{array}{l}\text { 12. Being a person on the } \\
\text { autistic spectrum is not } \\
\text { important to my sense of } \\
\text { what kind of person I am.* }\end{array}$ & .04 & .47 & .23 & .67 \\
\hline $\begin{array}{l}\text { 16. In general, being a person } \\
\text { on the autistic spectrum is an } \\
\text { important part of my self- } \\
\text { image. }\end{array}$ & .34 & .10 & .53 & .78 \\
\hline
\end{tabular}

Notes. Factor loadings based on principal components extraction with oblimin rotation; items numbered with original 18 -item scale numbering; factor loadings over .40 in bold; * indicates reverse scored item. 
Table 6. Inter-construct correlation matrix

\begin{tabular}{lcccc}
\hline & $\begin{array}{c}\text { Involvement in } \\
\text { online } \\
\text { community }\end{array}$ & $\begin{array}{c}\text { Communication } \\
\text { preference }\end{array}$ & $\begin{array}{c}\text { Autistic } \\
\text { Identity }\end{array}$ & $\begin{array}{c}\text { Self- } \\
\text { Determination }\end{array}$ \\
$\begin{array}{l}\text { Autistic adults } \\
\text { Involvement in online community }\end{array}$ & 1 & 1 & & \\
Communication preference & 0.13 & -0.05 & 1 & \\
Autistic identity & $.33^{* *}$ & -0.04 & $.36^{* *}$ & 1 \\
Self-Determination & $.38^{* *}$ & & & \\
Non-autistic adults & 1 & & & \\
Involvement in online community & 0.25 & 1 & & 1 \\
Communication preference & $.37^{* *}$ & -0.11 & & \\
Self-Determination & & &
\end{tabular}

Notes. ${ }^{*} p<.05 ;{ }^{* *} p<.01$ 
Table 7. Relationship between involvement in the online Autistic community and Autistic identity and their dimensions

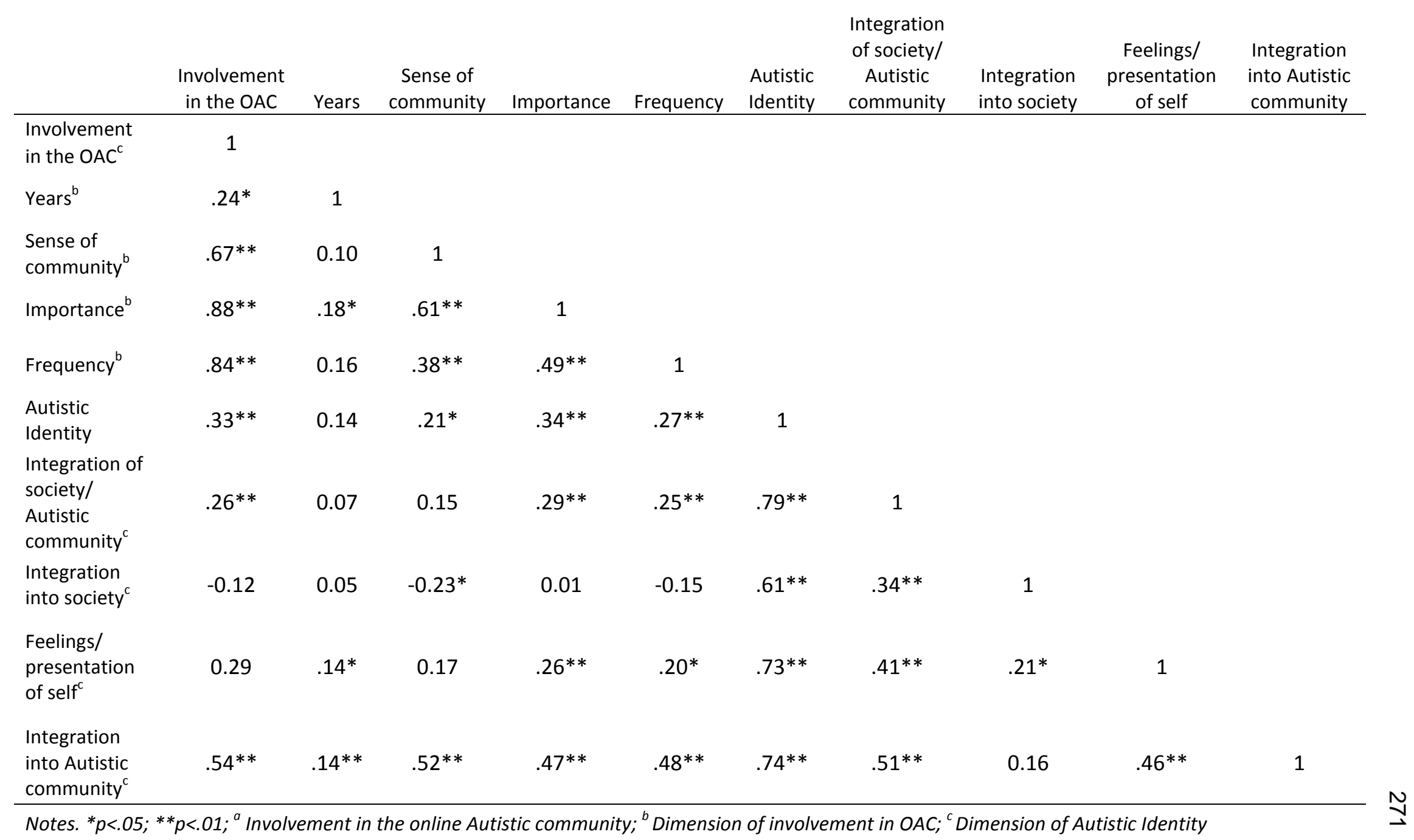


Table 8. Research Questions, Hypotheses, and Findings

RQ 1: How is involvement in the online Autistic community related to Autistic identity, and how does communication preference moderate the relationship?

\begin{tabular}{|l|l|l|l|}
\hline \multicolumn{2}{|l|}{ Description } & Analysis & Findings \\
\hline $\mathrm{H}$ & $\begin{array}{l}\text { Involvement in the online Autistic community will } \\
\text { be positively related to Autistic identity. }\end{array}$ & $\begin{array}{l}\text { Correlation } \\
\text { community was significantly positively related to Autistic } \\
\text { identity. }\end{array}$ \\
\hline $\begin{array}{l}\text { Communication preference will moderate the } \\
\text { relationship between involvement in the online } \\
\text { Autistic community and Autistic identity, with a } \\
\text { more positive relationship between involvement } \\
\text { in the online Autistic community and Autistic } \\
\text { identity when a person prefers online } \\
\text { communication over when a person does not } \\
\text { prefer online communication. }\end{array}$ & $\begin{array}{l}\text { Moderated } \\
\text { regression }\end{array}$ & $\begin{array}{l}\text { Only significant individual predictor was involvement in the } \\
\text { online Autistic community: } \beta=.32, t(109)=3.49, p<.01,95 \% \\
C l=.12, .43\end{array}$ \\
\hline
\end{tabular}

RQ 2: How is involvement in the online Autistic community related to self-determination, and how does communication preference moderate the relationship?

\begin{tabular}{|l|l|l|l|}
\hline \multicolumn{2}{|l|}{ Description } & Analysis & Findings \\
\hline $\mathrm{H}$ & $\begin{array}{l}\text { Involvement in the online Autistic community will } \\
\text { be positively related to self-determination. }\end{array}$ & Correlation & $\begin{array}{l}n=113, \mathrm{r}=.38, \mathrm{p}<.01 ; \text { Involvement in the online Autistic } \\
\text { community was significantly positively related to self- } \\
\text { determination. }\end{array}$ \\
\hline $\mathrm{H}$ & $\begin{array}{l}\text { Communication preference will moderate the } \\
\text { relationship between involvement in the online } \\
\text { Autistic community and self-determination, with a } \\
\text { more positive relationship between involvement }\end{array}$ & $\begin{array}{l}\text { Moderated } \\
\text { regression }\end{array}$ & $\begin{array}{l}\text { Only significant individual predictor was involvement in the } \\
\text { online Autistic community: } \beta=.30, t(109)=2.20, p<.01,95 \% \mathrm{Cl}=\end{array}$ \\
\hline
\end{tabular}




\begin{tabular}{|l|l|l|l|}
\hline $\begin{array}{l}\text { in the online Autistic community and self- } \\
\text { determination when a person prefers online } \\
\text { communication over when a person does not } \\
\text { prefer online communication. }\end{array}$ & & $.011, .22$ \\
\hline
\end{tabular}

RQ 3: Does communication preference have a stronger moderating effect between involvement in the online Autistic community and selfdetermination than between involvement in the online community and self-determination in non-autistic adults?

\begin{tabular}{|c|c|c|c|}
\hline \multicolumn{2}{|c|}{ Description } & \multirow[b]{2}{*}{$\begin{array}{c}\text { Analysis } \\
\\
\text { Three-way } \\
\text { moderated } \\
\text { regression }\end{array}$} & \multirow[b]{2}{*}{\begin{tabular}{|l|} 
Findings \\
Overall model was significant: $F(6,240)=11.71, p<.05, f^{2}=.33$ \\
Significant individual predictors were involvement in the \\
online Autistic community: $\beta=.39, t(246)=6.39, p<.05,95 \%$ \\
$\mathrm{Cl}=.110, .208$; communication preference: $\beta=.16$, \\
$t(246)=2.54, p<.01,95 \% \mathrm{Cl}=.027, .211$; and preference type: \\
$\beta=-.20, t(246)=-3.26, p<.01,95 \% \mathrm{Cl}=-.121,-.030$ (no \\
interactions were significant)
\end{tabular}} \\
\hline H 3 & $\begin{array}{l}\text { Communication preference will have a stronger } \\
\text { moderating effect on the relationship between } \\
\text { involvement in the online community and self- } \\
\text { determination in autistic adults than non-autistic } \\
\text { adults. }\end{array}$ & & \\
\hline
\end{tabular}

RQ 4: How are involvement in the online Autistic community, Autistic identity, and self-determination (autonomy, competence, and relatedness) related among autistic individuals, and do these relationship differ between individuals who prefer online communication compared to autistic individuals who do not prefer online communication?

\begin{tabular}{|c|c|c|c|}
\hline \multicolumn{2}{|c|}{ Description } & Analysis & Findings \\
\hline $\begin{array}{l}\mathrm{H} \\
4.1\end{array}$ & $\begin{array}{l}\text { The Autistic Identity Scale containing the factors of } \\
\text { integration into society, integration into the } \\
\text { Autistic community, internal integration of } \\
\text { similarities and differences with others, and } \\
\text { integration of internal feelings of self with } \\
\text { presentation of self is a plausible representation }\end{array}$ & $\begin{array}{l}\text { Exploratory } \\
\text { factor analysis; } \\
\text { structural } \\
\text { equation model } \\
\text { (SEM) }\end{array}$ & $\begin{array}{l}\text { Overall modified scale } \alpha=.84 \text {; subscales: } \\
\text { Integration into society ( } \alpha=.71 \text { ) } \\
\text { - Unstandardized estimate }=.40, \mathrm{SE}=.13, \mathrm{CR}=3.18, \mathrm{p}<.01 \text {; } \\
\text { standardized regression weight }=.30 \\
\text { Integration into the Autistic community }(\alpha=.70) \\
\text { - Unstandardized estimate }=.96, \mathrm{SE}=.17, \mathrm{CR}=5.65, \mathrm{p}<.01 ; \\
\text { standardized regression weight }=.75\end{array}$ \\
\hline
\end{tabular}




\begin{tabular}{|c|c|c|c|}
\hline & $\begin{array}{l}\text { of the dimensions of Autistic identity adapted } \\
\text { from the disability identity theory of Gill (1997). } \\
\text { Specifically, the scale and subscales will exhibit } \\
\text { acceptable internal consistency reliability } \\
\text { (Cronbach's alpha of .70), and the four factors of } \\
\text { the Autistic Identity Scale will have a positive } \\
\text { significant regression weights on Autistic identity } \\
\text { in the proposed structural model. }\end{array}$ & & 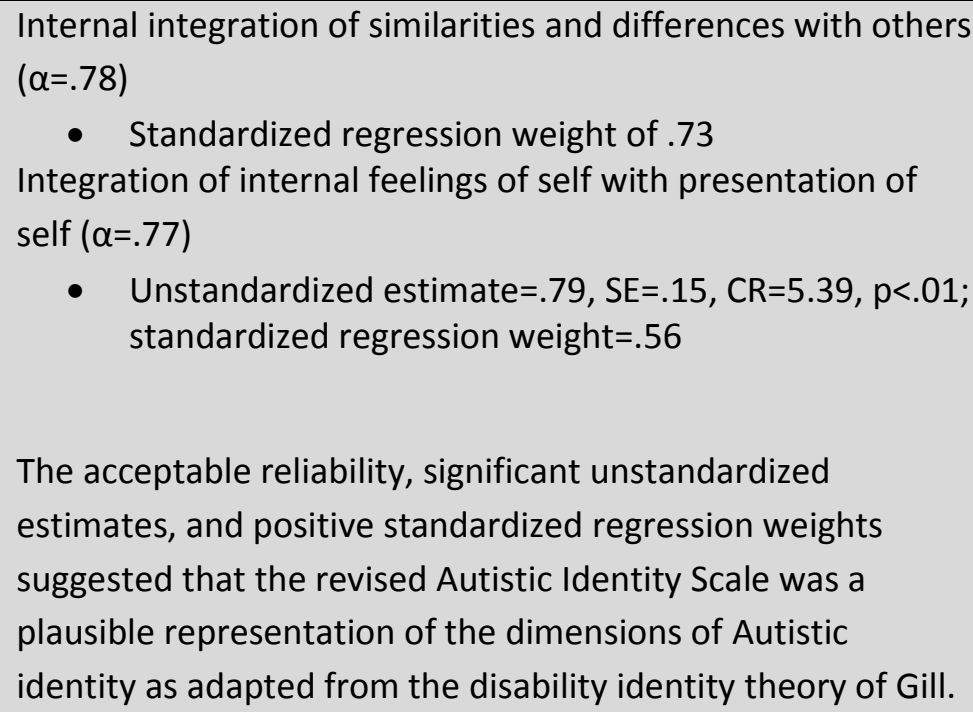 \\
\hline $\begin{array}{l}\mathrm{H} \\
4.2\end{array}$ & $\begin{array}{l}\text { Involvement in the online Autistic community will } \\
\text { be positively related to Autistic identity in autistic } \\
\text { adults who prefer online communication. }\end{array}$ & \multirow{3}{*}{$\begin{array}{l}\text { SEM (configural } \\
\text { model of adults } \\
\text { who prefer } \\
\text { online } \\
\text { communication) }\end{array}$} & $\begin{array}{l}\text { Covariance between involvement in the online Autistic } \\
\text { community and Autistic identity was significant, cov }=.24 \text {, } \\
\mathrm{SE}=.08, \mathrm{CR}=2.94, \mathrm{p}<.01, r=.48 \text {. Involvement in the online } \\
\text { Autistic community was significantly positively related to } \\
\text { Autistic identity in autistic adults who prefer online } \\
\text { communication. }\end{array}$ \\
\hline $\begin{array}{l}\mathrm{H} \\
4.3\end{array}$ & $\begin{array}{l}\text { Involvement in the online Autistic community will } \\
\text { be positively related to self-determination in } \\
\text { autistic adults who prefer online communication. }\end{array}$ & & $\begin{array}{l}\text { Covariance between involvement in the online Autistic } \\
\text { community and self-determination was significant, cov }=.09 \text {, } \\
\mathrm{SE}=.04, \mathrm{CR}=2.28, \mathrm{p}=.02, r=.28 \text {. Involvement in the online } \\
\text { Autistic community was significantly positively related to self- } \\
\text { determination in autistic adults who prefer online } \\
\text { communication. }\end{array}$ \\
\hline $\begin{array}{l}\mathrm{H} \\
4.4\end{array}$ & $\begin{array}{l}\text { Involvement in the online Autistic community will } \\
\text { be positively related to self-determination in }\end{array}$ & & $\begin{array}{l}\text { Covariance between Autistic identity and self-determination } \\
\text { was significant, } \operatorname{cov}=.12, \mathrm{SE}=.05, \mathrm{CR}=2.69, \mathrm{p}=.01, \mathrm{r}=.47 \text {. }\end{array}$ \\
\hline
\end{tabular}




\begin{tabular}{|c|c|c|c|}
\hline & autistic adults who prefer online communication. & & $\begin{array}{l}\text { Involvement in the online Autistic community was } \\
\text { significantly positively related to self-determination in } \\
\text { autistic adults who prefer online communication. }\end{array}$ \\
\hline $\begin{array}{l}H \\
4.5\end{array}$ & $\begin{array}{l}\text { Involvement in the online Autistic community will } \\
\text { be positively related to Autistic identity in autistic } \\
\text { adults who do not prefer online communication. }\end{array}$ & $\begin{array}{c}\text { SEM } \\
\text { (comparison }\end{array}$ & $\begin{array}{l}\text { The relationship between involvement in the online Autistic } \\
\text { community and Autistic identity was significant, cov=.30, } \\
\mathrm{SE}=.14, \mathrm{CR}=2.14, \mathrm{p}=.03, \mathrm{r}=.33 \text {. Involvement in the online } \\
\text { Autistic community was significantly positively related to } \\
\text { autistic adults who do not prefer online communication. }\end{array}$ \\
\hline $\begin{array}{l}\mathrm{H} \\
4.6\end{array}$ & $\begin{array}{l}\text { Involvement in the online Autistic community will } \\
\text { be positively related to self-determination in } \\
\text { autistic adults who do not prefer online } \\
\text { communication. }\end{array}$ & $\begin{array}{l}\text { model of autistic } \\
\text { adults who do } \\
\text { not prefer online }\end{array}$ & $\begin{array}{l}\text { The relationship between involvement in the online Autistic } \\
\text { community was not significantly related to self- } \\
\text { determination in the sample cov }=.004, S E=.05, C R=.10, p=.92 \text {, } \\
r=.01 \text {. }\end{array}$ \\
\hline $\begin{array}{l}\mathrm{H} \\
4.7\end{array}$ & $\begin{array}{l}\text { Autistic identity will be positively related to self- } \\
\text { determination in autistic adults who do not prefer } \\
\text { online communication. }\end{array}$ & $\mathrm{cc}$ & $\begin{array}{l}\text { The relationship between Autistic identity and self- } \\
\text { determination was significant, cov }=.14, S E=.05, C R=2.69 \text {, } \\
p<.01, r=.47 \text {. Autistic identity was significantly positively } \\
\text { related to self-determination in autistic adults who do not } \\
\text { prefer online communication. }\end{array}$ \\
\hline $\begin{array}{l}\mathrm{H} \\
4.8\end{array}$ & $\begin{array}{l}\text { The relationships among involvement in the online } \\
\text { Autistic community, Autistic identity, and self- } \\
\text { determination will be stronger in autistic adults } \\
\text { who prefer online communication than in autistic } \\
\text { adults who do not prefer online communication. }\end{array}$ & $\begin{array}{l}\text { SEM (difference } \\
\text { between } \\
\text { configural and } \\
\text { comparison } \\
\text { models) }\end{array}$ & $\begin{array}{l}\text { No significant difference in overall model fit between the two } \\
\text { groups, } \chi^{2}(d f=6)=6.98, p=.32 \text {. However, the nonsignificant } \\
\text { relationship of involvement in the online Autistic community } \\
\text { and self-determination was only nonsignificant in autistic } \\
\text { adults who do not prefer online communication. Therefore, } \\
\text { the hypothesis was partially supported. }\end{array}$ \\
\hline
\end{tabular}

Note: Table rows that are shaded grey indicate that analysis findings in those rows supported the corresponding hypotheses.

Hypothesis 4.8 was partially supported, so that row is shaded with a lighter grey. 
Table 9. Moderation of communication preference on the relationship between involvement in the online Autistic community and Autistic identity

\begin{tabular}{|c|c|c|c|c|c|c|c|c|}
\hline & Adjusted $\mathrm{R}^{2}$ & B & SE B & $\beta$ & $\begin{array}{l}95 \% \mathrm{Cl} \\
\text { Lower } \\
\text { Bound }\end{array}$ & $\begin{array}{l}95 \% \mathrm{Cl} \\
\text { Upper } \\
\text { Bound }\end{array}$ & Effect size $f^{2}$ & Power \\
\hline $\begin{array}{l}\text { Involvement in online } \\
\text { community }^{1}\end{array}$ & & 0.28 & 0.08 & 0.32 & 0.119 & 0.433 & \multirow{3}{*}{0.16} & \multirow{3}{*}{0.94} \\
\hline Communication preference & 0.11 & -0.12 & 0.08 & -0.14 & -0.266 & 0.031 & & \\
\hline Interaction term & & 0.10 & 0.08 & 0.11 & -0.058 & 0.256 & & \\
\hline
\end{tabular}

Note. ${ }^{1}$ Involvement in the Online Autistic Community 
Table 10. Relationship between involvement in the online Autistic community and self-determination and their dimensions Involvement in the

online Autistic Years of Sense of Importance of Frequency of Self-

community involvement community involvement involvement determination Autonomy Competence Relatedness

Involvement in the

online Autistic

community

1

Years of involvement

Sense of community

Importance of

involvement

Frequency of

involvement

Self-determination

Autonomy

$.24 *$

$.67^{* *}$

1

Competence

$.88^{* *}$

0.10

1

Relatedness

$84 * *$

$.18^{*}$

$.61^{* *}$

1

Notes. ${ }^{*} p<.05 ;{ }^{* *} p<.01$

0.16

0.10

0.01

0.01

$-0.15$

0.12

$.38^{* *}$

$.56 * *$

$-0.05$

$-0.03$

$.87^{* *}$
1

0.13

0.11

$-0.11$

$.44^{* *}$
1

$.61 * * \quad 1$

$.62 * * \quad .21 * \quad 1$

$\begin{array}{lll}.68 * * & 0.02 & -0.06\end{array}$ 
Table 11. Moderation of communication preference on the relationship between involvement in the online Autistic community and self-determination

\begin{tabular}{|c|c|c|c|c|c|c|c|c|}
\hline & $\begin{array}{c}\text { Adjusted } \\
\mathrm{R}^{2}\end{array}$ & B & SE B & $\beta$ & $\begin{array}{l}95 \% \mathrm{Cl} \\
\text { Lower } \\
\text { Bound }\end{array}$ & $\begin{array}{l}95 \% \mathrm{Cl} \\
\text { Upper } \\
\text { Bound }\end{array}$ & $\begin{array}{l}\text { Effect } \\
\text { size } f^{2}\end{array}$ & Power \\
\hline $\begin{array}{l}\text { Involvement in } \\
\text { online community }^{1}\end{array}$ & & 0.11 & 0.05 & 0.30 & 0.011 & 0.216 & \multirow{3}{*}{.19} & \multirow{3}{*}{.97} \\
\hline $\begin{array}{l}\text { Communication } \\
\text { preference }\end{array}$ & 0.12 & -0.01 & 0.07 & -0.01 & -0.133 & 0.126 & & \\
\hline Interaction term & & 0.05 & 0.07 & 0.10 & -0.084 & 0.188 & & \\
\hline
\end{tabular}

Note. ${ }^{1}$ Involvement in the Online Autistic Community 
Table 12. Moderation of communication preference and population type (autistic, non-autistic) on the relationship between involvement in the online Autistic community and self-determination

\begin{tabular}{|c|c|c|c|c|c|c|c|c|}
\hline & $\begin{array}{c}\text { Adjusted } \\
\mathrm{R}^{2}\end{array}$ & B & SE B & $\beta$ & $\begin{array}{l}95 \% \mathrm{Cl} \\
\text { Lower } \\
\text { Bound }\end{array}$ & $\begin{array}{l}95 \% \mathrm{Cl} \\
\text { Upper } \\
\text { Bound }\end{array}$ & $\begin{array}{l}\text { Effect } \\
\text { size } f^{2}\end{array}$ & Power \\
\hline $\begin{array}{l}\text { Involvement in } \\
\text { online community }^{1}\end{array}$ & & 0.16 & 0.03 & 0.39 & 0.110 & 0.208 & & \\
\hline $\begin{array}{l}\text { Communication } \\
\text { preference }\end{array}$ & & 0.12 & 0.05 & 0.16 & 0.027 & 0.211 & & \\
\hline Population type & & -0.08 & 0.02 & -0.20 & -0.121 & -0.030 & & \\
\hline $\begin{array}{l}\text { Involvement } \mathrm{x} \\
\text { Communication } \\
\text { preference term }\end{array}$ & 0.21 & -0.06 & 0.05 & -0.07 & -0.160 & 0.038 & 0.33 & 0.99 \\
\hline $\begin{array}{l}\text { Involvement x Pop } \\
\text { type term }\end{array}$ & & -0.02 & 0.02 & -0.04 & -0.063 & 0.034 & & \\
\hline $\begin{array}{l}\text { Three-way } \\
\text { interaction term }\end{array}$ & & 0.02 & 0.05 & 0.03 & -0.079 & 0.121 & & \\
\hline
\end{tabular}

Notes. ${ }^{1}$ Involvement in the Online Autistic Community; ${ }^{2}$ Two-way interaction term of involvement in the online Autistic community and communication preference; ${ }^{3}$ Two-way interaction term of involvement in the online Autistic community and population type (autistic, non-autistic; ${ }^{4}$ Three-way interaction term of involvement in the online Autistic community, communication preference, and population type (autistic, non-autistic) 
Table 13. Fit indices for the structural equation models

\begin{tabular}{|c|c|c|c|c|c|c|}
\hline Fit index & $\begin{array}{c}\text { Fit } \\
\text { indicator/ } \\
\text { cut-off }^{1} \\
\end{array}$ & $\begin{array}{c}\text { Proposed } \\
\text { model }^{2} \\
\end{array}$ & $\begin{array}{c}\text { Final } \\
\text { model }^{3}\end{array}$ & $\begin{array}{c}\text { Prefer online } \\
\text { communication }\end{array}$ & $\begin{array}{l}\text { Do not prefer } \\
\text { online } \\
\text { communication }\end{array}$ & $\begin{array}{c}\text { Comparison } \\
\text { model }^{4}\end{array}$ \\
\hline $\begin{array}{l}\text { Chi- } \\
\text { square }\end{array}$ & n.s. & $22.85^{*}$ & $14.50^{*}$ & $14.67^{*}$ & 6.57 & 6.98 \\
\hline RMSEA & $<.10$ & .11 & .08 & .12 & .01 & \\
\hline PCLOSE & $>.05$ & .03 & .16 & .09 & .59 & \\
\hline SRMR & $<.08$ & .06 & .05 & .06 & .05 & \\
\hline $\mathrm{CFI}$ & $>.90$ & .90 & .95 & .93 & 1.00 & \\
\hline \multicolumn{7}{|c|}{ Fit index difference } \\
\hline $\operatorname{NFI}(\Delta-1)$ & & & & & & .04 \\
\hline $\mathrm{IFI}(\Delta-2)$ & & & & & & .04 \\
\hline $\operatorname{RFI}(\mathrm{P}-1)$ & & & & & & -.03 \\
\hline TLI(P-2) & & & & & & -.03 \\
\hline
\end{tabular}

Notes. ${ }^{*} p<.05 .{ }^{1}$ The acceptable fit indicator or cut-off value that the literature recommends to indicate model fit. See the Results section for sources of cut-off recommendations. ${ }^{2}$ Includes all autistic adults in model and covariance among the variables involvement in the online Autistic community, Autistic identity, and self-determination. ${ }^{3}$ Includes the same sample and variables as proposed model with added covariance between two dimensions of Autistic identity. ${ }^{4}$ Compares the differences between the models of autistic adults who prefer online communication and do not prefer online communication. 
Figure 1. AASPIRE's academic and community partner contribution to the CBPR process

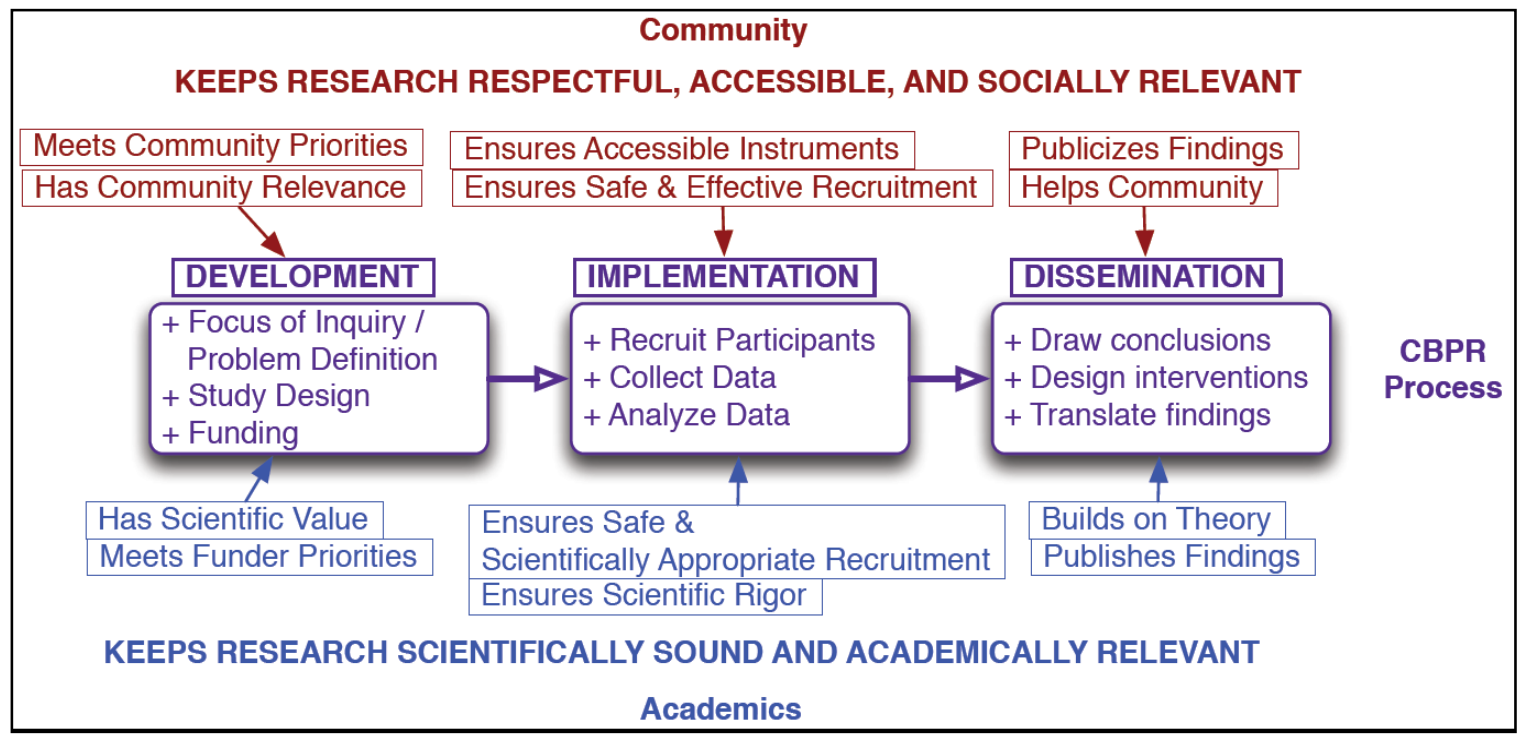

(Nicolaidis, Raymaker, McDonald, Dern, Ashkenazy, Boisclair, Robertson, \& Baggs, 2011) 
Figure 2. Communication preferences moderating the relationship between involvement in the online Autistic community and Autistic identity

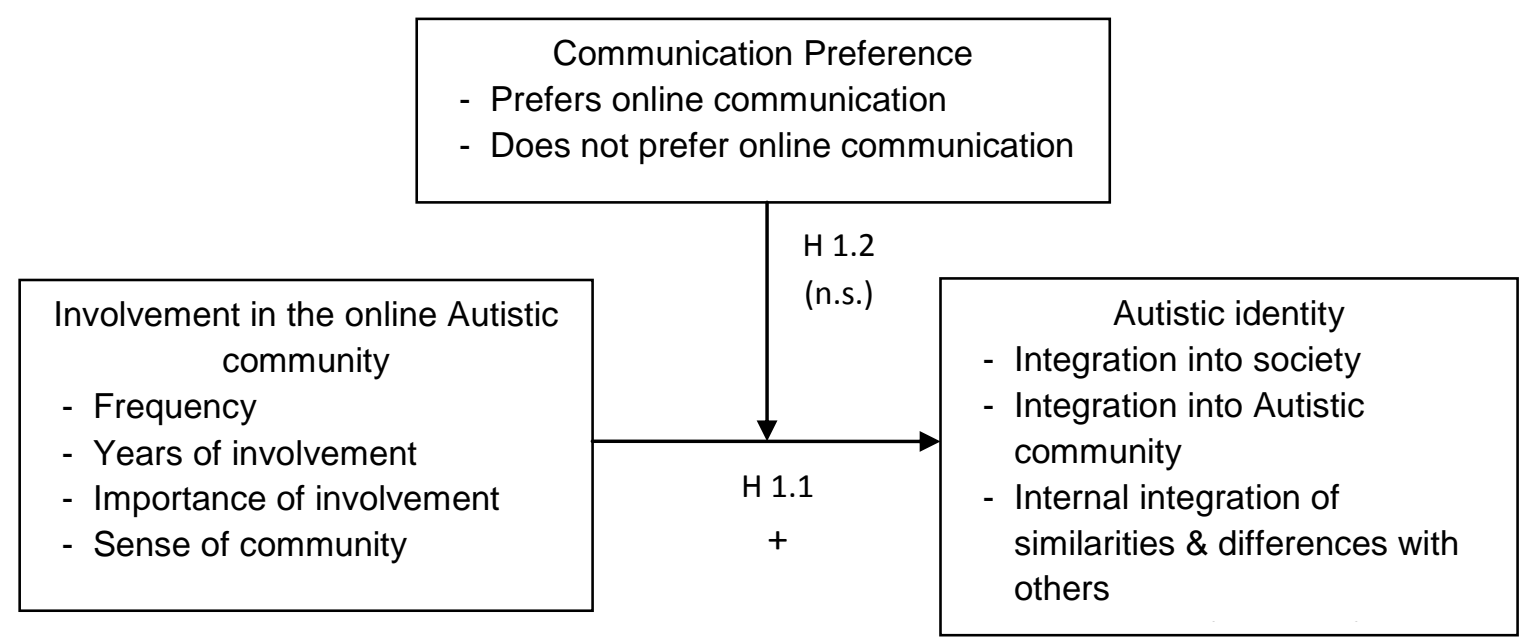


Figure 3. Communication preferences moderating the relationship between involvement in the online Autistic community and self-determination

\begin{tabular}{|c|c|c|c|c|}
\hline & \multicolumn{3}{|c|}{$\begin{array}{l}\text { Communication Preference } \\
\text { - Prefers online communication } \\
\text { - Does not prefer online communication }\end{array}$} & \\
\hline & & \multirow{2}{*}{$\begin{array}{l}\text { H } 2.2 \\
\text { (n.s.) }\end{array}$} & & \\
\hline \multirow{2}{*}{\multicolumn{2}{|c|}{$\begin{array}{l}\text { Involvement in the online Autistic } \\
\qquad \text { community } \\
\text { - Frequency } \\
\text { - Years of involvement } \\
\text { - Importance of involvement } \\
\text { - Sense of community }\end{array}$}} & & \multirow{2}{*}{\multicolumn{2}{|c|}{$\begin{array}{l}\text { Self-Determination } \\
\text { - Autonomy } \\
\text { - Competence } \\
\text { - Relatedness }\end{array}$}} \\
\hline & & $\begin{array}{l}2.1 \\
+\quad\end{array}$ & & \\
\hline
\end{tabular}


Figure 4. Communication preference and population type (autistic, non-autistic) moderating the relationship between involvement in the online Autistic community and self-determination

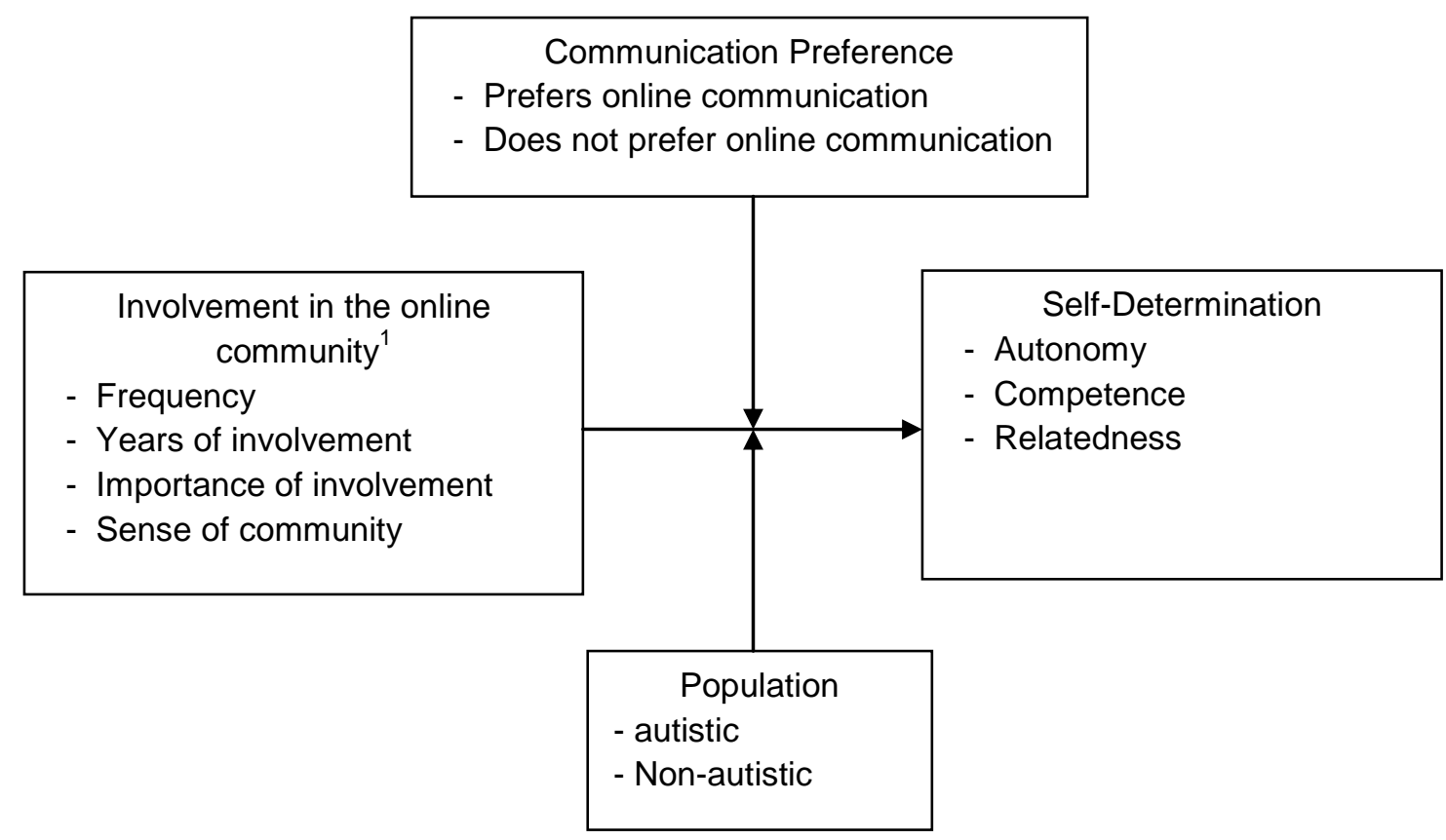

Notes. ${ }^{1}$ The online community refers to the online Autistic community for autistic participants and any online community for non-autistic participants. 
Figure 5. Hypothesized conceptual model for the comparison of relationships among involvement in the online Autistic community, Autistic identity, and self-determination by communication preference

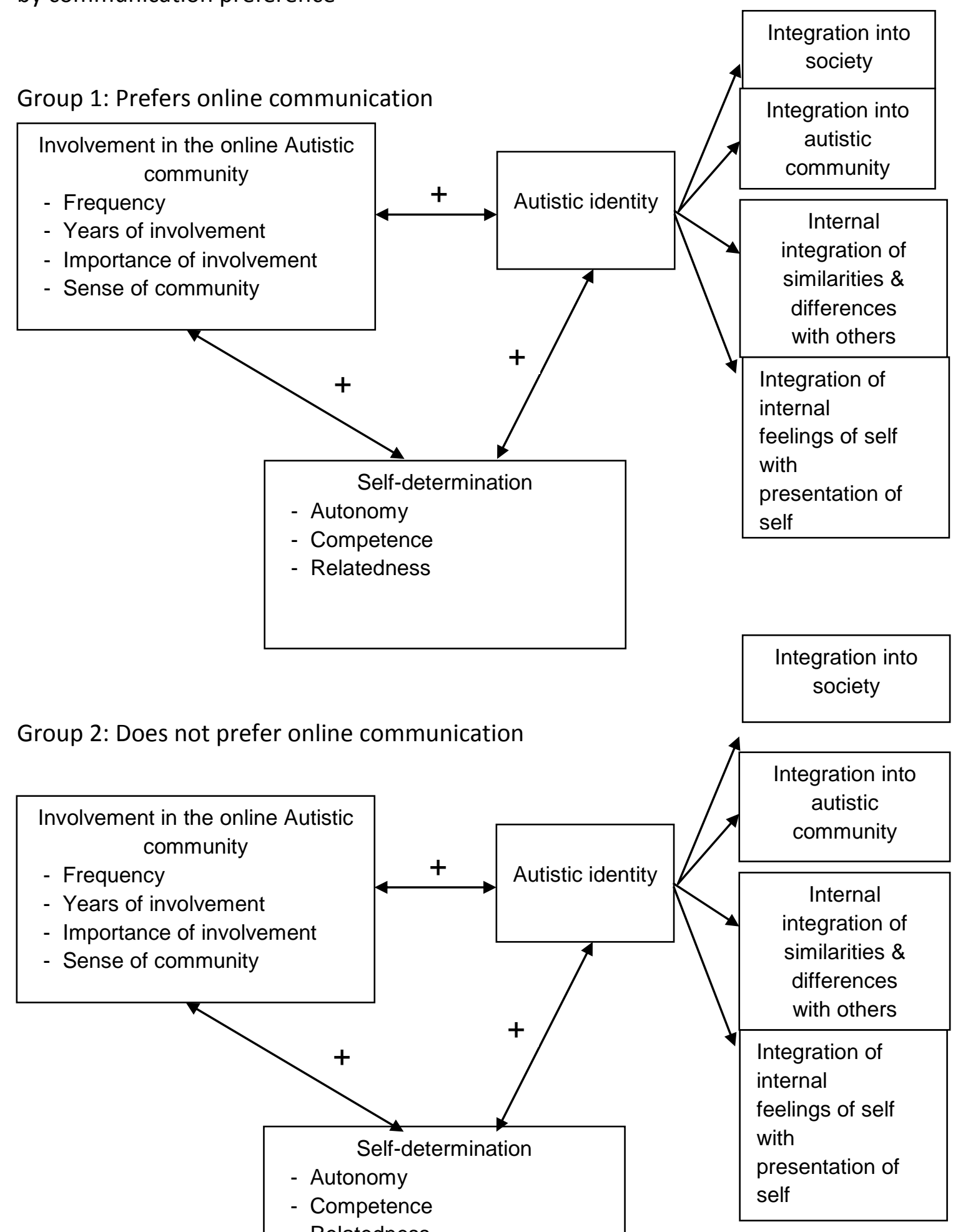


Figure 6. Participants and measures of the Gateway Survey utilized in the AASPIRE Internet Use, Community, and Well-Being Study

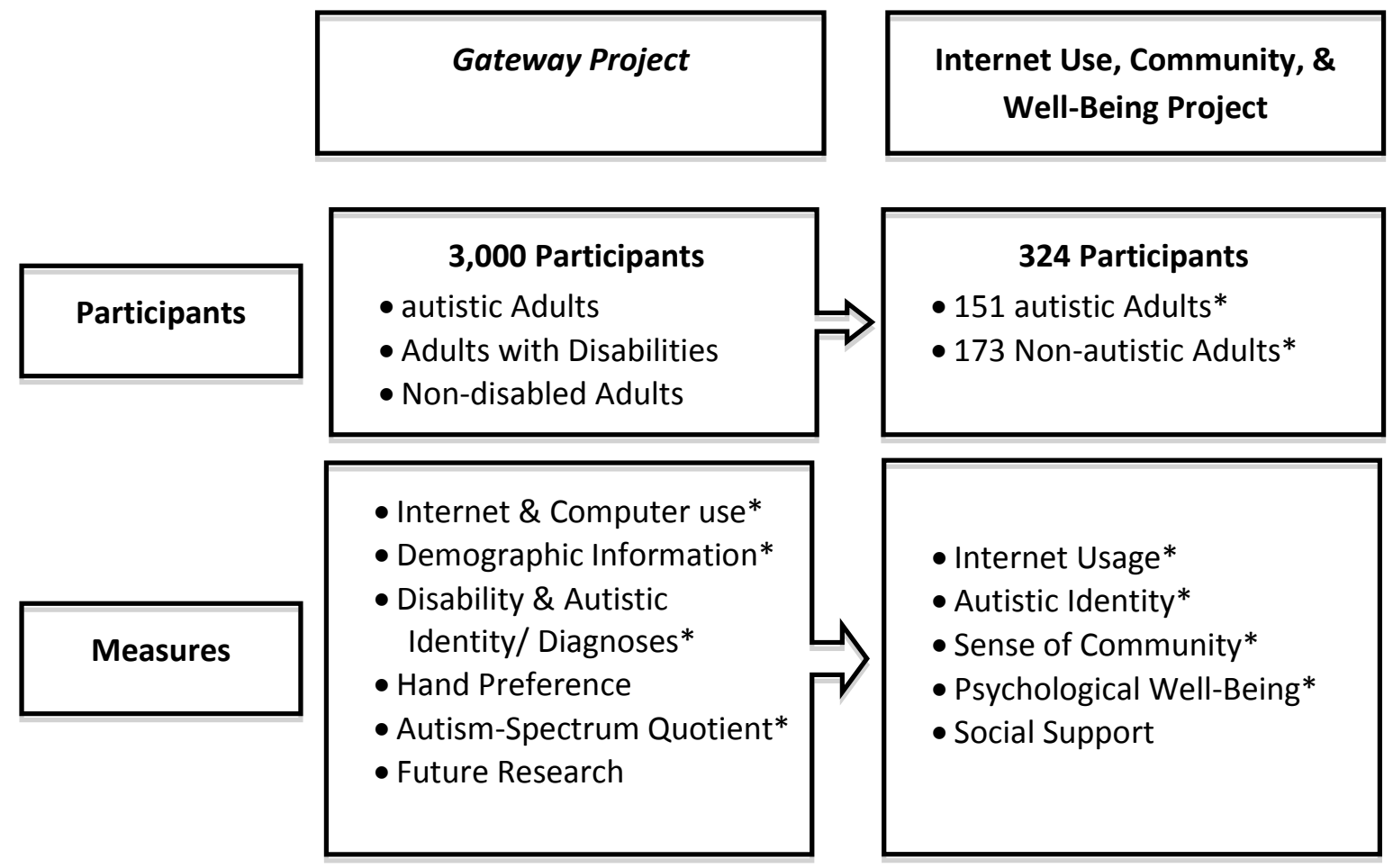

Note: Asterisked $\left({ }^{*}\right)$ items indicate use in the current study 
Figure 7. Structural model utilized for the comparison of relationships among involvement in the online Autistic community, Autistic Identity, and self-determination in autistic adults who prefer online communication and autistic adults who do not prefer online communication

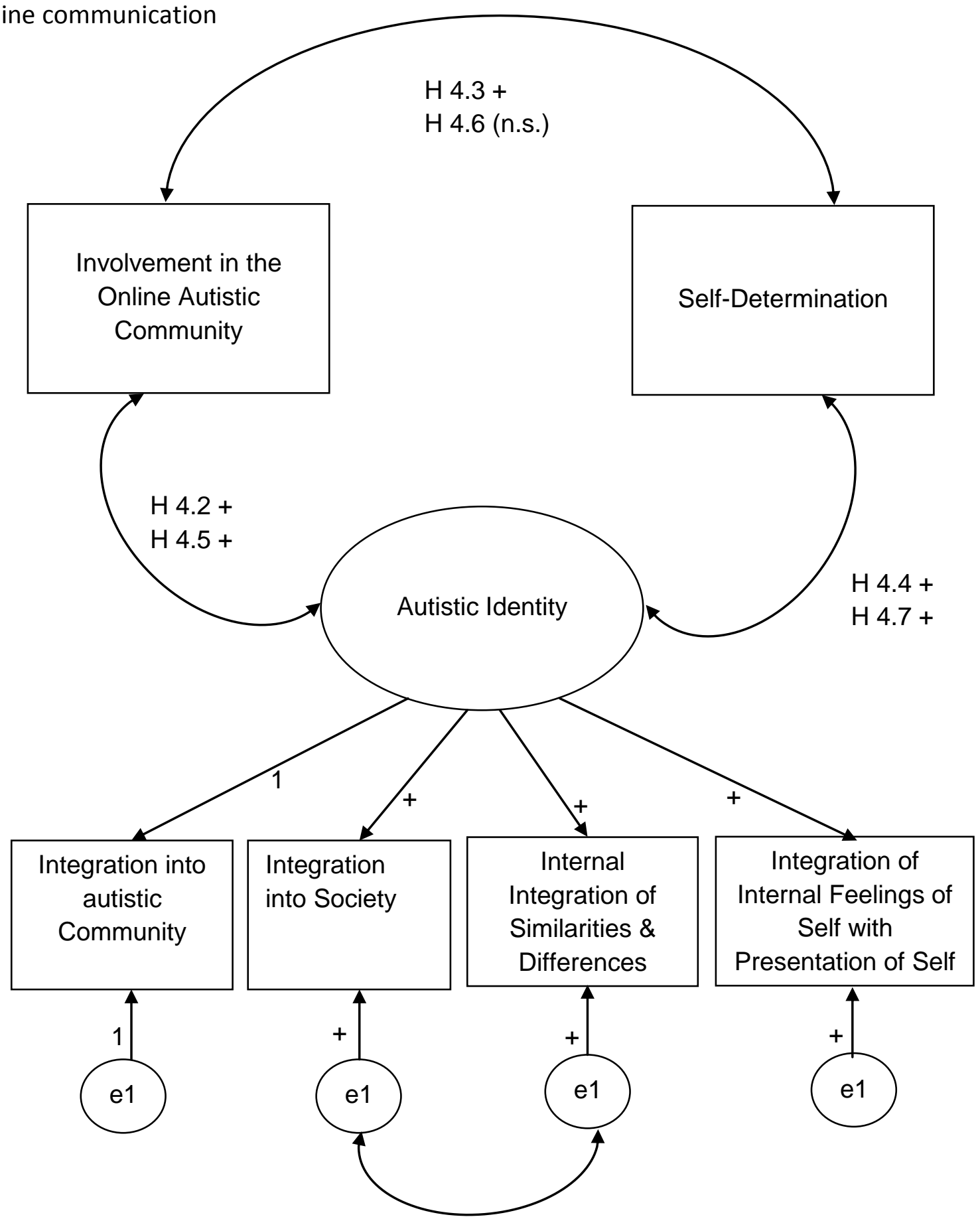




\section{References}

Abberley, P. (1987). The concept of oppression and the development of a social theory of disability. Disability, Handicap \& Society, 2, 5-19.

Adams, G. R., \& Marshall, S. K. (1996). A developmental social psychology of identity: Understanding the person-in-context. Journal of Adolescence, 19, 429-442.

Aiken, L., \& West, S. (1991). Multiple regression: Testing and interpreting interactions. Newbury Park, CA: Sage.

Algozzine, B., Browder, D., Karvonen, M., Test, D. W., \& Wood, W. M. (2001). Effects of Interventions to Promote Self-Determination for Individuals With Disabilities. Review of Educational Research, 71(2), 219-277. doi:10.3102/00346543071002219

Allen, R. F., \& Allen, J. (1987). A sense of community, a shared vision and a positive culture: core enabling factors in successful culture based health promotion. American Journal of Health Promotion, 1(3), 40-47.

Archakis, A., \& Tzanne, A. (2005). Narrative positioning and the construction of situated identities: Evidence from conversations of a group of young people in Greece. Narrative Inquiry. Retrieved from http://www.ingentaconnect.com/content/jbp/nari/2005/00000015/00000002/art 00004

Asperger, H. (1944). "autistic psychopathy" in childhood. In U. Frith (Ed.), Autism and Aspergers Syndrome. Cambridge, MA: Cambridge.

Association, A. P. (2000). Diagnostic and Statistical Manual of Mental Disorders, Fourth Edition. Washington, DC: American Psychiatric Association.

Baard, P., Deci, E., \& Ryan, R. (2004). Intrinsic Need Satisfaction : A Motivational Basis of Performance and Well-Being in Two Work Settings 1. Journal of Applied Social Psychology, 34(10), 2045-2068.

Bachrach, Kenneth, M., \& Zautra, A. J. (1985). Coping with a community stressor: The threat of a hazardous waste facility. Journal of Health and Social Behavior, 127141.

Bagatell, N. (2007). Orchestrating voices: autism, identity and the power of discourse. Disability \& Society, 22(4), 413-426. doi:10.1080/09687590701337967 
Bagatell, N. (2010). From Cure to Community: Transforming Notions of Autism Historical Trends Influencing the Emergence of an Autism Community. Ethos, 38(1), 33-55. doi:10.1111/j.1548-1352.2009.01080.x.34

Baker, D. L. (2006). Neurodiversity, neurological disability and the public sector: notes on the autism spectrum. Disability \& Society, 21(1), 15-29. doi:10.1080/09687590500373734

Ballaban-Gil, K., Rapin, I., Tuckman, R., \& Shinnar, S. (1996). Longitudinal examination of the behavioral, language, and social changes in a population of adolescents and young adults with autistic disorder. Pediatruc Neurology, 15(3), 217-223.

Barak, A., \& Sadovski, Y. (2008). Internet use and personal empowerment of hearingimpaired adolescents. Computers in Human Behavior, 24, 1802-1815.

Barnard, J., Harvey, V., Potter, D., \& Prior, A. (2001). Ignored or ineligible? The reality for adults with autism spectrum disorders. The National autistic Society Report for Autism Awareness Week. London. Retrieved from http://www.autism.org.uk/nas/jsp/polopoly.jsp?d=160\&a=3463

Baron-Cohen, S, Wheelwright, S., Skinner, R., Martin, J., \& Clubley, E. (2001). The Autism-Spectrum Quotient (AQ): Evidence from Asperger Syndrome/ highfunctioning autism, males and females, scientists and mathematicians. Journal of Autism and Developmental Disorders, 31, 5-17.

Baron-Cohen, Simon. (2002). The extreme male brain theory of autism. Trends in cognitive sciences, 6(6), 248-254. Retrieved from http://www.ncbi.nlm.nih.gov/pubmed/21094892

Barrett, M. (2006). "Like dynamite going off in my ears": Using autobiographical accounts of autism with teaching professionals. Educational Psychology in Practice, 22(2), 95-110.

Bart, S., \& Maarten, V. (2011). When is Identity Congruent with the Self? A SelfDetermination Theory Perspective. In K. Schwartz, K. Luyckx, \& V. Vignoles (Eds.), Hangbook of Identity Theory and Research (pp. 381-402). New York: Springer.

Benford, P., \& Standen, P. J. (2009). The Internet: A comfortable communication medium for people with Asperger syndrome (AS) and high functioning autism (HFA)? Journal of Assistive Technologies, 3(2), 44-53. 
Berkman, L. F., Glass, T., Seeman, I., \& Brisette, T. E. (2000). From social integration to health: Durkheim in the new millennium. Social Science and Medicine, 51, 843-857.

Bierer, L.N. (2013). Online community building by autistic adults (Doctoral Dissertation) Retrieved from repositories.lib.utexas.edu.

Biklen, D. (2005). Autism and the myth of the person alone. New York: NYU Press.

Bishop, D. V. M. (1989). Autism, Asperger's syndrome and semantic-pragmatic disorders: Where are the boundaries? British Journal of Disorders of Communication, 24, 107-121.

Blatt, B., \& Kaplan, F. (1974). Christmas in Purgatory: A Photographic Essay on Mental Retardation. Syracuse, NY: Human Policy Press.

Blume, H. (1997a). Autistics, freed from face-to-face encouters, are communicating in cyberspace. New York Times. Retrieved January 28, 2009, from http://www.nytimes.com/library/cyber/techcol/063097techcol.html

Blume, H. (1997b). Autism \& the Internet or -"It"s the wiring, stupid"'. New York Times. Retrieved January 28, 2009, from http://web.mit.edu/commforum/papers/blume.html

Boase, J., Horrigan, J. B., Wellman, B., \& Rainie, L. (2006). The Strength of Internet Ties The Internet and email aid users in maintaining their social networks and provide pathways to help when people face big decisions Findings. Washington, DC.

Boundy, K. (2008). "Are you sure, sweetheart, that you want to be well?": An exploration of the neurodiversity movement. A Journal of Psychology, Politics, \& Radicalism, 7, 1-16.

Bowker, N., \& Tuffin, K. (2002). Disability discourses for online identities. Disability \& Society Society, 17(3), 327-344. doi:10.1080/0968759022013988

Boykin, A. W. (1986). The triple quandary and the schooling of Afro-American children. In U. Neisser (Ed.), The School Achievement of Minority Children (pp. 57-92). Hillsdale, NJ: Erlbaum.

Bradley, E. A., Summers, J. A., Wood, H. L., \& Bryson, S. E. (2004). Comparing rates of psychiatric and behavior disorders in adolescents and young adults with severe intellectual disability with and without autism. Journal of Autism \& Developmental Disorders, 34(2), 151-161. 
Brisenden, S. (1986). Independent living and the medical model of disability. Disability, Handicap \& Society, 1(2), 173-178.

Broderick, A. A., \& Ne'eman, A. (2008). Autism as metaphor: narrative and counter-narrative. International Journal of Inclusive Education, 12(5-6), 459-476. doi:10.1080/13603110802377490

Brownlow, C. (2007a). The Construction of the autistic Individual: Investigations in Online Discussion Groups. University of Brighton. Retrieved from http://eprints.brighton.ac.uk/3073/

Brownlow, C. (2007b). The Construction of the autistic Individual: Investigations in Online Discussion Groups. University of Brighton.

Brownlow, C. (2010). Presenting the self: negotiating a label of autism. Journal of intellectual \& developmental disability, 35(1), 14-21.

doi:10.3109/13668250903496336

Brownlow, C., \& O'Dell, L. (2002). Ethical Issues for Qualitative Research in On-line Communities. Disability \& Society, 17(6), 685-694. doi:10.1080/0968759022000010452

Brownlow, C., \& O’Dell, L. (2006). Constructing an Autistic Identity: AS voices online. Mental retardation, 44(5), 315-21. doi:10.1352/00476765(2006)44[315:CAAIAV]2.0.CO;2

Brugha, T. S., McManus, S., Bankart, J., Scott, F., Purdon, S., Smith, J., \& Meltzer, H. (2011). Epidemiology of autism spectrum disorders in adults in the community in England. Archives of General Psychiatry, 68, 459-465.

Brydon-Miller, M. (2010). Participatory Action Research: Psychology and Social Change. Journal of Social Issues, 53(4), 657-666. doi:10.1111/j.1540-4560.1997.tb02454.x

Burke, M., Kraut, R., \& Williams, D. (2010). Social use of computer-mediated communication by adults on the autism spectrum. ACM Conference on Computer Supported Cooperative Work (pp. 425-434). New York City, NY.

Burks, M. A., Pardos, J. L., Waddell, L., \& Nakane, M. (2000). The Internet and people with disabilities: Expanding horizons or barrier to information and services. Retrieved April 22, 2009, from http://www.isoc.org/inet2000/cdproceedings/5c/5c_1.htm 
Cagle, S. E. (2006). Self-determination and life satisfaction among transition-age students with disabilities. University of Southern California.

Caplan, S. E. (2005). A Social Skill Account of Problematic Internet Use. Journal of Communication, 55(4), 721-736. doi:10.1093/joc/55.4.721

Caplan, Scott E. (2003). Preference for Online Social Interaction: A Theory of Problematic Internet Use and Psychosocial Well-Being. Communication Research, 30(6), 625-648. doi:10.1177/0093650203257842

Caplan, Scott E. (2010). Theory and measurement of generalized problematic Internet use: A two-step approach. Computers in Human Behavior, 26(5), 1089-1097. doi:10.1016/j.chb.2010.03.012

Carter, R. (2006). Solutions for missing data in structural equation modeling. Research \& Practice in Assessment, 1(1), 1-6. Retrieved from http://www.kokdemir.info/courses/psi748/docs/[p] Solutions for Missing Data in SEM.pdf

Cass, V. C. (1984). Homosexuals identity formation: Testing a theoretical model. Journal of Sex Research, 20(2), 143-167.

Centers for Disease Control and Prevention; CDC/ATSDR Committee on Community Engagement (2011). Principles of community engagement $\left(2^{\text {nd }}\right.$ ed). Retrieved September 20, 2014 from http://www.atsdr.cdc.gov/communityengagement/pdf/pce_report_508_final.pdf.

Chamak, B. (2008). Autism and social movements: French parents' associations and international autistic individuals' organisations. Sociology of health \& illness, 30(1), 76-96. doi:10.1111/j.1467-9566.2007.01053.x

Chambers, C. R., Wehmeyer, M. L., Saito, Y., Lida, K. M., \& Lee, Y. (n.d.). Exceptionality : A Special Self-Determination : What Do We Know ? Where Do We Go ?, (November 2012), 37-41.

Chavis, D. M., Hogge, J. H., McMillan, D. W., \& Wandersman, A. (1986). Sense of community through Brunswik's lens: A first look. Journal of Community Psychology, $14,24-40$.

Chavis, D. M., Lee, K. S., \& Acosta, J. D. (2008). The Sense of Community (SCI) Revised: The Reliability and Validity of the SCI-2. 2nd International Community Psychology Conference. Lisboa, Portugal. 
Chipuer, H. M., \& Pretty, G. M. H. (1999). A review of the Sense of Community Index: Current uses, factor structure, reliability, and further development. Journal of Community Psychology, 27(6), 643-658.

Clarke, J., \& Van Amerom, G. (2007). 'Surplus suffering ': Differences between organizational understandings of Asperger's syndrome and those people who claim the 'disorder'. Disability \& Society, 22, 761-776.

Cobb, B., Lehmann, J., Newman-gonchar, R., \& Alwell, M. (2009). Self-Determination for Students with Disabilities. Career Development and Transition for Exceptional Individuals, 32(2), 108-114.

Committee., U. D. of H. \& H. S. I. A. C. (2009). The Interagency Autism Coordinating Committee strategic plan for autism spectrim disorder research. Retrieved April 22, 2009, from http://iacc.hhs.gov/reports/2009/iacc-strategic-plan-for-autismspectrum-disorder-research-jan26.shtml

Cramer, E. P., \& Gilson, S. F. (1999). Queers and crips: Parallel identity development processes for persons with nonvisible disabilities and lesbian, gay, and bisexual persons. Journal of Gay, Lesbian, and Bisexual Identity, 4(1), 23-37.

Cromby, J., \& Standon, P. (1999). Cyborgs and stigma: Technology, disability, subjectivy. In A. J. Gordo-Lopez \& I. Parker (Eds.), Cyberpsychology (pp. 95-112). New York City, NY: Routledge.

Cronbach, L. J. (1951). Coefficient alpha and the internal structure of tests. Psychometrika, 16(3), 297-334.

Cross, W. E. (1971). Toward a psychology of black liberation: The negro-to-black conversion experience. Black World Journal, 20(9), 13-27.

D'Augelli, A. R. (1994). Identity development and sexual orientation: Toward a model of lesbian, gay, and bisexual development. In Edison J Trickett, R. J. Watts, \& D. Birman (Eds.), Human diversity: Perspectives on people in context (pp. 312-333). San Francisco: Jossey-Bass Publishers.

Dalton, J. H., Elias, M. J., \& Wandersman, A. (2000). Community Psychology: Linking Individuals and Communities. Belmont, CA: Wadsworth Printing.

Davidson, J. (2008). Autistic culture online: virtual communication and cultural expression on the spectrum. Social \& Cultural Geography, 9(7), 791-806. doi:10.1080/14649360802382586 
Davidson, J., \& Henderson, V. L. (2010). "Coming out" on the spectrum: autism, identity and disclosure. Social \& Cultural Geography, 11(2), 155-170.

doi:10.1080/14649360903525240

Deci, E. L., \& Ryan, R. M. (1985). The General Causality Orientations Scale: Self-

Determination in Personality. Journal of Research in Personality, 19, 109-134.

Deci, E. L., \& Vansteenkiste, M. (2004). Self-determination Theory and Basic Need Satisfaction: Understanding Human Development in Positive Psychology. Ricerche di Psicologia, 27(1), 23-40.

Development, N. I. of C. H. and H. (2005). Autism overview: What we know. Retrieved April 12, 2009, from http://www.nichd.nih.gov/autism.

Dybwad, G., \& Bersani, H. (1996). New Voices: Self-Advocacy by People with Disabilities. Cambridge, MA: Brookline Books.

Dye, L., Hendy, S., Hare, D. J., \& Burton, M. (2004). Capacity to consent to participate in research-a recontextualization. British Journal of Learning Disabilities, 32(3), 144150.

Erickson, W., \& Lee, C. (2008). 2007 Disability Status Report: United States. Ithaca, NY.

Erikson, E. H. (1956). The problem of ego identity. Journal of the American Psychoanalytic Association, 4, 56-121.

Fabrigar, L. R., Wegener, D. T., MacCallum, R. C., \& Strahan, E. J. (1999). Evaluating the use of exploratory factor analysis in psychological research. Psychological Methods, 4(3), 272-299. doi:10.1037//1082-989X.4.3.272

Farley, M. A., McMahon, W. M., Fombonne, E., Jenson, W. R., Miller, J., Gardner, M., Block, H., et al. (2009). Twenty-year outcomes for individuals with autism and average or near-average cognitive abilities. Autism Research, 2, 1-10.

Fawcett, S. B., White, G. W., Balcazar, F. E., Suarez-Balcazar, Y., Matthews, R. M., PaineAndrews, A., Seekins, T., et al. (1994). A contextual-behavioral model of empowerment: Case studies involving people with physical disabilities. American Journal of Community Psychology, 22(4).

Field, S., \& Hoffman, A. (1999). The importance of family involvement for promoting self-determination in adolescents with autism and other developmental disabilities. Focus on Autism and Other Developmental Disabilities, 14(1), 36-41. 
Filipek, P. A., Accardo, P. J., Baranek, G. T., Cook Jr., E. H., Dawson, G., Gordon, B., Gravel, J. S., et al. (1999). The screening and diagnosis of autism spectrum disorders. Journal of Autism and Developmental Disorders, 29(6), 439-484.

Fine, M. (1992). Disruptive Voices: The Possibilities of Feminist Research. Ann Arbor, MI: University of Michigan Press.

Fine, M., \& Asch, A. (1988). Disability beyond stigma: Social interaction, discrimination, and activism. Journal of Social Issues, 44, 3-21.

Folstein, S. E. (1999). Autism. First-hand accounts of emotional experiences in autism: A qualitative analysis, 11, 269-277.

Ford, J., MacCallum, R., \& Tait, M. (2006). The application of exploratory factor analysis in applied psychology: A critical review and analysis. Personnel Psychology.

Retrieved from http://onlinelibrary.wiley.com/doi/10.1111/j.1744-

6570.1986.tb00583.x/abstract

Forster, P. M. (2004). Psychological Sense of Community in Groups on the Internet. Behavior Change, 21(2), 141-146.

Foucault, M. (1978). The History of Sexuality: An Introduction. New York City, NY: Random House, Inc.

French, K. (1993). My personal holiday. Our Voice, the newsletter of Autism Network International, 1(3).

Friere, P. (1972). Pedagogy of the Oppressed. New York: Continuum.

Gallup, I. (2009). Computers and the Internet. Retrieved from http://www.gallup.com/poll/1591/Computers-Internet.aspx

Giles, D.C. (2014). 'DSM-V is taking our identity': The reaction of the online community to the proposed changes in the diagnosis of Asperger's disorder. Health, 18(2) 179195. doi: $10.1177 / 1363459313488006$

Gill, C. (1997). Four Types of Integration in Disability Identity Development. Journal of Vocational Rehabilitation. Retrieved from http://psycnet.apa.org/psycinfo/199804936-004

Gill, C. J. (1997). Disability Identity Scale. Chicago, IL. 
Gordon, B. O., \& Rosenblum, K. E. (2001). Bringing disability into the sociological frame: A comparison of disability with race, sex, and sexual orientation statuses. Disability \& Society, 16(1), 5-19. doi:10.1080/0968759002002083

Grinker, R. R. (2007). Unstrange Minds: Remapping the World of Autism. Cambridge, MA: Basic Books.

Gross, E. F., Juvonen, J., \& Gable, S. L. (2002). Internet Use and Well-Being in Adolescence. Journal of Social Issues, 58(1), 75-90. doi:10.1111/1540-4560.00249

Guo, B., Bricout, J. C., \& Huang, J. (2005). A common open space or a digital divide? A social model perspective on the online disability community in China. Disability \& Society, 20(1), 49-66.

Hand, M. A. C. (2011). The Use of YouTube by Persons with Asperger's Syndrome for Online Social Networking. Valdosta State University.

Happé, F., \& Ronald, A. (2008). The 'fractionable autism triad ': a review of evidence from behavioural, genetic, cognitive and neural research. Neuropsychology Review, 18, 287-304.

Havercamp, S. M., Scandlin, D., \& Roth, M. (2004). Health disparities among adults with developmental disabilities, adults with other disabilities, and adults not reporting disability in North Carolina. Public Health Reports, 119, 418-426.

Helson, R., \& Srivastava, S. (2001). Three paths of adult development: Conservers, seekers, and achievers. Journal of Personality and Social .... Retrieved from http://darkwing.uoregon.edu/ sanjay/pubs/threepaths.pdf

Hoekstra, R. A., Bartels, M., Cath, D. C., \& Boomsma, D. I. (2008). Factor structure, reliability, and criterion validity of the Autism-Spectrum Quotient (AQ): A study in Dutch population and patient groups. Journal of Autism \& Developmental Disorders, 38(8), 1555-1566.

Howard, P., Rainie, L., \& Jones, S. (2001). Days and Nights on the Internet The Impact of a Diffusing Technology. American Behavioral Scientist, 45(3), 382-404. Retrieved from http://abs.sagepub.com/content/45/3/383.short

Howlin, P., Goode, S., Hutton, J., \& Rutter, M. (2004). Adult outcome for children with autism. Journal of child psychology and psychiatry, and allied disciplines, 45(2), 212-29. Retrieved from http://www.ncbi.nlm.nih.gov/pubmed/14982237 
Hurlbutt, K., \& Chalmers, L. (2002). Adults with autism speak out: Perceptions of their life experiences. Focus on Autism and Other Developmental Disabilities, 17, 103111.

Huta, V., \& Ryan, R. M. (2009). Pursuing Pleasure or Virtue: The Differential and Overlapping Well-Being Benefits of Hedonic and Eudaimonic Motives. Journal of Happiness Studies, 11(6), 735-762. doi:10.1007/s10902-009-9171-4

IACC/OARC Autism Spectrum Disorder Research Publications Analysis Report: The Global Landscape of Autism Research. (2012). Retrieved from http://iacc.hhs.gov/publications-analysis/july2012/index.shtml

Israel, B. a, Schulz, a J., Parker, E. a, \& Becker, a B. (1998). Review of community-based research: assessing partnership approaches to improve public health. Annual review of public health, 19, 173-202. doi:10.1146/annurev.publhealth.19.1.173

Israel, B. A. (2000). "Community-Based Participatory Research: Principles, Rationale and Policy Recommendations" Successful Models of Community-Based Participatory Research (pp. 16-22). Washington, DC.

Jaarsma, P., \& Welin, S. (2012). Autism as a natural human variation: reflections on the claims of the neurodiversity movement. Health care analysis : HCA : journal of health philosophy and policy, 20(1), 20-30. doi:10.1007/s10728-011-0169-9

Jensen, V. K. (2002). Treatment of Autism in Young Children : Behavioral Intervention and Applied Behavior Analysis, 14(4), 42-52.

Johnston, M. M., \& Finney, S. J. (2010). Measuring basic needs satisfaction: Evaluating previous research and conducting new psychometric evaluations of the Basic Needs Satisfaction in General Scale. Contemporary Educational Psychology, 35(4), 280-296. doi:10.1016/j.cedpsych.2010.04.003

Jones, R. S., \& Meldal, T. O. (2001). Social relationships and Asperger's syndrome: A qualitative analysis of first-hang accounts. Journal of Learning Disabilities, 5(1), 3541.

Jones, R. S., Zahl, A., \& Huws, J. C. (2001). First-hand accounts of emotional experiences in autism: A qualitative analysis. Disability \& Society, 16(3), 393-401.

Jordan, C. J. (2010). Evolution of autism support and understanding via the World Wide Web. Intellectual and developmental disabilities, 48(3), 220-7. doi:10.1352/19349556-48.3.220 
Josselson, R. (1987). Finding herself: Pathways to identity development in women. San Francisco, London: Jossey-Bass Publishers.

Kanner, L. (1943). Autistic disturbances of affective contact. Nervous child. Retrieved from http://mail.neurodiversity.com/library_kanner_1943.pdf

Kasser, V., \& Ryan, R. M. (1999). The Relationship of Psychological Needs for Autonomy and Relatedness to Vitality, Well-Being, and Mortality in a Nursing Home. Journal of Applied Social Psychology, 29(5), 935-954.

Katz, J., Rice, R., \& Acord, S. (2004). Personal mediated communication and the concept of community in theory and practice. Communication ..., 315-371. Retrieved from http://www.socialtext.net/data/workspaces/socialmediaberkeley/attachments/syll abus:20070616034611-0-4033/original/community.online.love.rice.doc

Khobzi, N., \& Flicker, S. (2010). Lessons learned from undertaking community-based participatory research dissertations: The trials and triumphs of two junior health scholars. Progress in Community Health Partnerships: Research, Education, and Action, 4 (4), 347-356.

Kidney, C. A. (2012). Involvement in the Online Autistic Community, Identity, Community, and Well-Being.

Kidney, C., \& Stack, E. (2012). Student Reflections on the Challenges and Rewards of Community-Based Participatory Research with Adults with Disabilities. The Community Psychologist, 45(2), 11-13.

Kim, H., Kim, G. J., Park, H. W., \& Rice, R. E. (2007). Configurations of Relationships in Different Media: FtF, Email, Instant Messenger, Mobile Phone, and SMS. Journal of Computer-Mediated Communication, 12(4), 1183-1207. doi:10.1111/j.10836101.2007.00369.x

Kim, J., LaRose, R., \& Peng, W. (2009). Loneliness as the cause and the effect of problematic Internet use: the relationship between Internet use and psychological well-being. Cyberpsychology \& behavior : the impact of the Internet, multimedia and virtual reality on behavior and society, 12(4), 451-5.

doi:10.1089/cpb.2008.0327

Kim, Y. S., Leventhal, B. L., Koh, Y. J., Fombonne, E., Laska, E., Lim, E. C., \& Grinker, R. R. (2011). Prevalence of autism spectrum disorders in a total population sample. The American Journal of Psychiatry, 168(5), 1-9. 
Kitzinger, C. (1989). The Regulation of Lesbian Identities: Liberal Humanism as an Ideology of Social Control. In J. Shotter \& K. J. Gergen (Eds.), Texts of Identity (pp. 82-98). London: Sage.

Klawiter, M. (2012). Racing for the Cure, Walking Women, and Toxic Touring : Mapping Cultures of Action within the Bay Area Terrain of Breast Cancer * , 46(1), 104-126.

Kline, R. (1998). Principles and Pracatice of Structural Equation Modeling (2nd ed.). New York City, NY: Guilford Press.

Kras, J. F. (2009). The "Ransom Notes" Affair When the Neurodiversity Movement Came of Age. Disability Studies Quarterly, 30(1). Retrieved from http://dsqsds.org/article/view/1065/1254

Kraut, R., Kiesler, S., Boneva, B., Cummings, J., \& Helgeson, V. (2002). Internet paradox revisited. Journal of Social Issues. Retrieved from http://onlinelibrary.wiley.com/doi/10.1111/1540-4560.00248/full

Kraut, R., Patterson, M., \& Lundmark, V. (1998). Internet paradox: A social technology that reduces social involvement and psychological well-being? American ..., 53(9), 1017-1031. Retrieved from http://psycnet.apa.org/journals/amp/53/9/1017/

Lachapelle, Y., Wehmeyer, M. L., Haelewyck, M.-C., Courbois, Y., Keith, K. D., Schalock, R., Verdugo, M. a, et al. (2005). The relationship between quality of life and selfdetermination: an international study. Journal of intellectual disability research : JIDR, 49(Pt 10), 740-4. doi:10.1111/j.1365-2788.2005.00743.x

Lee, B. S., Lee, I. S., Rhee, M. K., Shin, E. K., \& Lim, M. H. (2008). Disability Identity According to the Severity of Disabilities in Persons with Spinal Cord Injury. Journal of Korean Academy of Rehabilitation Medicine, 32(5), 537-543.

Lee, J. (2012). Ethnic Identity as a Predictor of Locus of Control and Academic SelfEfficacy. Sentience, 7, 17-21. Retrieved from https://www.psych.umn.edu/sentience/files/Lee_2012.pdf

Lewin, K. (1946). Action research and minority problems. Journal of Social Issues, 2, 3446.

Lord, C., \& Schopler, E. (1985). Brief report: Differences in sex ratios in autism as a function of measured intelligence. Journal of Autism and Developmental Disorders, 15, 185-193. 
Luyckx, K., Soenens, B., Berzonsky, M. D., Smits, I., Goossens, L., \& Vansteenkiste, M. (2007). Information-oriented identity processing, identity consolidation, and wellbeing: The moderating role of autonomy, self-reflection, and self-rumination. Personality and Individual Differences, 43(5), 1099-1111. doi:10.1016/j.paid.2007.03.003

MacLeod, A., Lewis, A., \& Robertson, C. (2013). "Why should I be like bloody Rain Man?!" Navigating the Autistic Identity. British Journal of Special Education, 40(1), 41-49. doi:10.1111/1467-8578.12015

Malian, I., \& Nevin, A. (2002). A Review of Self-Determination Literature: Implications for Practitioners. Remedial and Special Education, 23(2), 68-74.

Marcia, J. E. (1966). Development and validation of ego-identity status. Journal of Personality and Social Psychology, 3(5), 551-558.

Mazurek, M. O. (2013). Social media use among adults with autism spectrum disorders. Computers in Human Behavior, 29(4), 1709-1714. doi:10.1016/j.chb.2013.02.004

McKenna, K., \& Bargh, J. (1998). Coming out in the age of the Internet: Identity“ demarginalization" through virtual group participation. Journal of personality and social ..., 75(3), 681-694. Retrieved from http://psycnet.apa.org/journals/psp/75/3/681/

McMillan, D. W. (1996). Sense of Community. Journal of Community Psychology, 24(4), 315-325.

McMillan, D. W., \& Chavis, D. M. (1986). Sense of community: A definition and theory. Journal of Community Psychology, 14(1), 6-23.

Merrick, E. (1999). Like Chewing Gravel: On the Experience of Analyzing Qualitative Research Findings Using a Feminist Epistemology. Psychology of Women Quarterly, 23, 47-57.

Montes, G., Halterman, J. S., \& Magyar, C. I. (2009). Access to and satisfaction with school and community health services for US children with ASD. Pediatrics, 124 Suppl , S407-13. doi:10.1542/peds.2009-1255L

Morgan, D. L. (1998). Practical Strategies for Combining Qualitative and Quantitative Methods: Applications to Health Research. Qualitative Health Research, 8(3), 362376. doi:10.1177/104973239800800307 
Murray, S. (2010). Autism Functions/The Function Of Autism. Disability Studies Quarterly, 30(1). Retrieved from http://dsq-sds.org/article/view/1048/1229

Newton, A. T., Kramer, A. D. I., \& McIntosh, D. N. (2009). Autism online: A comparison of Word Usage in Bloggers With and Without Autism Spectrum Disorders. ACM International Conference on Human Factors in Computing Systems. Boston, MA.

Nicolaidis, C., Raymaker, D., McDonald, K., Dern, S., Ashkenazy, E., Boisclair, C., Robertson, S., et al. (2011). Collaboration strategies in nontraditional communitybased participatory research partnerships: Lessons from an academic-community partnership with Autistic self-advocates. Progress in Community Health Partnerships: Research, Education, and Action, 5(2), 143-50. doi:10.1353/cpr.2011.0022

Nicolaidis, C., Raymaker, D., Mcdonald, K., Dern, S., Boisclair, W. C., Ashkenazy, E., \& Baggs, A. (2012). Healthcare disparities experienced by autistic adults who access the Internet. Journal of General Internal Medicine.

Nie, N. H., \& Erbrin, L. (2000). Internet and Society: A Preliminary Report. Stanford Institute for the Quantitative Study of Society. .... Retrieved from http://scholar.google.com/scholar?hl=en\&btnG=Search\&q=intitle:Internet+and+So ciety:+A+Preliminary+Report\#2

Njong, A. M., \& Ningaye, P. (2008). Characterizing weights in the measurement of multidimensional poverty: An application of data-driven approaches to Cameroonian data * OPHI Working Paper 21. OPHI Working Paper 21, (21).

Nota, L., Ferrari, L., Soresi, S., \& Wehmeyer, M. (2007). Self-determination, social abilities and the quality of life of people with intellectual disability. Journal of intellectual disability research : JIDR, 51(Pt 11), 850-65. doi:10.1111/j.13652788.2006.00939.x

O'brien, R. M. (2007). A Caution Regarding Rules of Thumb for Variance Inflation Factors. Quality \& Quantity, 41(5), 673-690. doi:10.1007/s11135-006-9018-6

O'Neil, S. (2008). The meaning of autism: beyond disorder. Disability \& Society, 23(7), 787-799. doi:10.1080/09687590802469289

Obst, P., Smith, S. G., \& Zinkiewicz, L. (2002). An exploration of sense of community, part 3: Dimensions and predictors of psychological sense of community in geographical communities. Journal of Community Psychology, 30(1), 119-133. 
Obst, P., \& White, K. (2004). Revisiting the sense of community index: A confirmatory factor analysis. Journal of Community Psychology, 32, 691-705.

Obst, P., \& White, K. (2005). An exploration of the interplay between psychological sense of community, social identification and salience. Journal of Community \& Applied Social Psychology, 15, 127-135.

Obst, P., Zinkiewicz, L., \& Smith, S. (2002a). Sense of community in science fiction fandom, Part 1: Understanding sense of community in an international community of interest. Journal of Community Psychology, 30(1), 87-103.

Obst, P., Zinkiewicz, L., \& Smith, S. (2002b). Sense of community in science fiction fandom, part 2: Comparing neighborhood and interest group sense of community. Journal of Community Psychology, 30(1), 105-117.

Oliver, M. (1992). Changing the social relations of research production? Disability, Handicap \& Society, 7(2), 101-114. Retrieved from http://www.tandfonline.com/doi/abs/10.1080/02674649266780141

Oliver, M. (1996). Defining impairment and disability: issues at stake. In C. Barnes \& G. Mercer (Eds.), Exploring the Divide (pp. 29-54). Leeds: The Disability Press.

Olney, M. F., \& Kim, A. (2001). Beyond Adjustment: Integration of cognitive disability into identity. Disability \& Society, 16(4), 563-583.

doi:10.1080/09687590120059540

Orsini, M., \& Smilth, M. (2010). Social movements, knowledge and public policy: the case of autism activism in Canada and the US. Critical Policy Studies, 4, 38-57.

Ortega, F. (2009a). The Cerebral Subject and the Challenge of Neurodiversity. BioSocieties, 4(4), 425-445. doi:10.1017/S1745855209990287

Ortega, F. (2009b). Disability, Autism, and Neurodiversity. Ciência \&amp; Saúde Coletiva, 14(1), 67-77. Retrieved from http://www.scielo.br/scielo.php?pid=S141381232009000100012\&amp;script=sci_arttext

Pledger, C. (2003). Discourse on disability and rehabilitation issue: Opportunity for psychology. American Psychologist, 58, 279-284.

Pollet, T. V, Roberts, S. G. B., \& Dunbar, R. I. M. (2011). Use of social network sites and instant messaging does not lead to increased offline social network size, or to 
emotionally closer relationships with offline network members. Cyberpsychology, behavior and social networking, 14(4), 253-8. doi:10.1089/cyber.2010.0161

Powers, L. E., Geenen, S., Powers, J., Pommier-Satya, S., Turner, A., Dalton, L. D., Drummond, D., et al. (2012). My Life: Effects of a longitudinal, randomized study of self-determination enhancement on the transition outcomes of youth in foster care and special education. Children and Youth Services Review, 34(11), 2179-2187. doi:10.1016/j.childyouth.2012.07.018

Putnam, R. D., \& Miller, J. J. (1995). Bowling Alone : The Collapse and Revival of American Community by.

Rappaport, J. (1977). Community psychology: Values, research and action. New York, NY: Holt, Rinehart \& Winston.

Reich, S. M., Subrahmanyam, K., \& Espinoza, G. (2012). Friending, IMing, and hanging out face-to-face: overlap in adolescents' online and offline social networks. Developmental psychology, 48(2), 356-68. doi:10.1037/a0026980

Ramdoss, S., Lang, R., Mulloy, A., Franco, J., O'Reilly, M., Didden, R., \& Lancioni, G. (2010) Use of computer-based interventions to teach communication skills to children with autism spectrum disorders: A systematic review. Journal of Behavioral Education, 20(1), 55-76.

Robertson, S. (2010). Neurodiversity, quality of life, and autistic adults: Shifting research and professional focuses onto real-life challenges. Disability Studies Quarterly, 30(1). Retrieved from http://dsq-sds.org/article/view/1069/1234

Robinson, W., \& Unruh, B. (2008). The Hepatitis Experiments at the Willowbrook State School. The Oxford Textbook of Clinical .... Retrieved from http://books.google.com/books?hl=en\&lr=\&id=IACroE8sE_MC\&oi=fnd\&pg=PA80\& $\mathrm{dq}=$ The+Hepatitis+Experiments+at+the+Willowbrook+State+School\&ots=d_2JcDO0 uP\&sig=92jtcsPXVGbOYmuXkPHRKBnnCec

Rohall, D. E., \& Cotten, S. R. (2002). Internet Use and the Self Concept: Linking Specific Uses to Global Self-Esteem. Current Research in Social Psychology, 8(1).

Romano, M., Osborne, L. a, Truzoli, R., \& Reed, P. (2013). Differential psychological impact of Internet exposure on Internet addicts. PloS one, 8(2), e55162. doi:10.1371/journal.pone.0055162 
Rosqvist, H. B., Brownlow, C., \& O’Dell, L. (2013). Mapping the social geographies of autism - online and off-line narratives of neuro-shared and separate spaces. Disability \& Society, 28(3), 367-379.

Ryan, R M, \& Deci, E. L. (2000). Self-determination theory and the facilitation of intrinsic motivation, social development, and well-being. The American psychologist, 55(1), 68-78. Retrieved from http://www.ncbi.nlm.nih.gov/pubmed/11392867

Ryan, Richard M., Huta, V., \& Deci, E. L. (2006). Living well: a self-determination theory perspective on eudaimonia. Journal of Happiness Studies, 9(1), 139-170. doi:10.1007/s10902-006-9023-4

Ryan, S., \& Räisänen, U. (2008). "It's like you are just a spectator in this thing": Experiencing social life the "aspie" way. Emotion, Space and Society, 1(2), 135-143. doi:10.1016/j.emospa.2009.02.001

Ryff, C. D. (1989). Happiness is everything, or is it? Explorations on the meaning of psychological well-being. Journal of Personaality and Social Psychology, 57, 10691081.

Salomon, D. (2010). From marginal cases to linked oppressions: Reframing the conflict between the autistic pride and animal rights movements. Journal for Critical Animal Studies, VIII(1), 47. Retrieved from http://www.criticalanimalstudies.org/wpcontent/uploads/2009/09/4-JCAS-Vol-VIII-Issue-I-and-II-2010-Essay-FROMMARGINAL-CASES-pp-47-72.pdf

Sarason, S. B. (1974). The Psychological Sense of Community: Prospects For a Community Psychology. San Francisco: Jossey-Bass.

Schur, L., Shields, T., \& Schriner, K. (2005). Generational cohorts, group membership, and political participation by people with disabilities. Political Research Quarterly, 58(3), 487-496.

Seymour, W., \& Lupton, D. (2004). Holding the line online: Exploring wired relationships for people with disabilities. Disability \& Society, 19(4), 291-305.

Shakespeare, T., \& Watson, N. (2002). The social model of disability: an outdated ideology? Research in Social Science and Disability, 2, 9-28.

Shapiro, J. (1994). No Pity: People with Disabilities Forging a New Civil Rights Movement. New York City, NY: Times Books. 
Sheldon, K. M., Ryan, R., \& Reis, H. T. (1996). What Makes for a Good Day? Competence and Autonomy in the Day and in the Person. PSPB, 22(12), 1270-1279.

Shipman, D. L., Sheldrick, R. C., \& Perrin, E. C. (2011). Quality of life in adolescents with autism spectrum disorders: Reliability and validity of self-reports. Journal of Developmental \& Behavioral Pediatrics, 32(2), 85-89.

Shirky, C. (2003). A group is its own worst enemy. Retrieved from http://www.shirky.com/writings/group_enemy.html

Shogren, K. a., Wehmeyer, M. L., Palmer, S. B., Soukup, J. H., Little, T. D., Garner, N., \& Lawrence, M. (2008). Understanding the Construct of Self-Determination:

Examining the Relationship Between the Arc's Self-Determination Scale and the American Institutes for Research Self-Determination Scale. Assessment for Effective Intervention, 33(2), 94-107. doi:10.1177/1534508407311395

Shore, S. M. (2004). Ask and Tell Self-Advocacy and Disclosure for People on the Autism Spectrum. Autism Asperger Pub Co. Retrieved from http://books.google.com/books?hl=en\&amp;Ir=\&amp;id=_rJ2XEveXoEC\&amp;oi=f nd\&amp;pg=PP1\&amp; dq=Ask+and+Tell+Self-

Advocacy+and+Disclosure+for+People+on+the+Autism+Spectrum\&amp;ots=nLFlef4VW\&amp;sig=hNhz81k6qCT83K3-4SjF6bq4luY

Siddiquee, A., \& Kagan, C. (2006). The Internet, empowerment, and identity: An exploration of participation by refugee women in a community Internet project (CIP) in the United Kingdom (UK). Journal of Community \& Applied Social Psychology, 16, 189-206.

Siebers, T. (2006). Disability Studies and the Future of Identity Politics. In L. M. Alcoff, M. Hames-Garcia, S. P. Mohanty, \& P. M. L. Moya (Eds.), Identity Politics Reconsidered (pp. 10-30). New York City, NY.

Silverman, C. (2008). Fieldwork on Another Planet: Social Science Perspectives on the Autism Spectrum. BioSocieties, 3(3), 325-341. doi:10.1017/S1745855208006236

Sinclair, J. (1993). DON'T MOURN FOR US. Our Voice, 1(3), 1-4. Retrieved from http://pepas.org/textos/dont_mourn_for_us.pdf

Sinclair, J. (1998). Is cure a goal? Retrieved from http://web.archive.org/web/20090331054458/http://web.syr.edu/ jisincla/cure.ht $\mathrm{m}$ 
Sinclair, J. (2005). Autism network international: the development of a community and its culture. Autism Network International: The Development of a .... Retrieved August 28, 2012, from http://www.autreat.com/History_of_ANI.html

Singer, A. (1999). Cure is not a four letter word.

Singh, J., Illes, J., Lazzeroni, L., \& Hallmayer, J. (2009). Trends in US autism research funding. Journal of Autism and Developmental Disorders2, 39, 788-795.

Snow, D. L., Grady, K., \& Goyette-Ewing, M. (2000). A perspective on ethical issues on community psychology. In J Rappaport \& E. Seidman (Eds.), Handbook of Community Psychology. New York: Kluwer Academic/Plenum Publishers.

Solomon, A. (2008). The Autism Rights Movement. New York Magazine, 25, 2008. Retrieved from http://www.pepas.org/textos/the_autism_rights_movement.pdf

Stroke, N. I. of N. D. and. (2009). Autism Fact Sheet. Retrieved June 4, 2008, from http://www.ninds.nih.gov/disorders/autism/detail_autism.htm

Suler, J. (1997). The Psychology of Cyberspace. Retrieved February 1, 2009, from http://www-usr.rider.edu/ suler/psycyber/psycyber.html

Sutherland, G., M.A., C., \& lacono, T. (2002). Health issues for adults with developmental disability. Research in Developmental Disabilities, 23, 422-445.

Tabachnick, B. G., \& Fidell, L. S. (1996). Using Multivariate Statistics (3rd ed.). New York, NY: HarperCollins Publishers.

Tager-Flusberg, H., Paul, R., \& Lord, C. (2005). Language and communication in autism. Handbook of autism and ..., 335-364. Retrieved from https://medicine.yale.edu/childstudy/autism/class/339_99903_Chapter 12 Language and communication in autism.pdf

Tajfel, H., \& Turner, J. C. (1979). An integrative theory of intergroup conflict. In W. G. Austin \& S. Worchel (Eds.), The Social Psychology of Intergroup Relations. Montery, CA: Brooks/Cole Publishing Company.

Tate, D., \& Pledger, C. (2003). An integrative conceptual framework of disability: New directions for research. American Psychologist, 58, 289-295.

Tavakol, M., \& Dennick, R. (2011). Making sense of Cronbach's alpha. International Journal of Medical Education, 2, 53-55. doi:10.5116/ijme.4dfb.8dfd 
Thomas, K. C., Ellis, A. R., McLaurin, C., Daniels, J., \& Morrissey, J. P. (2007). Access to care for autism-related services. Journal of autism and developmental disorders, 37(10), 1902-12. doi:10.1007/s10803-006-0323-7

Towards a common language for functioning, disability and health: ICF. (2002).Geneva: World Health Organization. Geneva. Retrieved from http://scholar.google.com/scholar?hl=en\&btnG=Search\&q=intitle:Towards+a+Com mon+Language+for+Functioning,+Disability+and+Health+ICF\#O

Townley, G., Kloos, B., Green, E. P., \& Franco, M. M. (2011). Reconcilable differences? Human diversity, cultural relativity, and sense of community. American journal of community psychology, 47(1-2), 69-85. doi:10.1007/s10464-010-9379-9

Trickett, E.J., Kelly, J. G., \& Vincent, T. A. (1985). The Spirit of Ecological Inquiry in Community Research. In E. C. Susskind \& D. C. Klein (Eds.), Community Research: Methods, Paradigms, and Applications (pp. 283-333). New York: Praeger.

Turkle, S. (1995). Life On the Screen: Identity in the Age of the Internet. New York: Simon \& Schuster.

Valkenburg, P. M., \& Peter, J. (2007). Preadolescents' and adolescents' online communication and their closeness to friends. Developmental psychology, 43(2), 267-277.

Vlachopoulos, S. P., \& Michailidou, S. (2006). Development and Initial Validation of a Measure of Autonomy, Competence, and Relatedness in Exercise: The Basic Psychological Needs in Exercise Scale. Measurement in Physical Education and Exercise Science, 10(3), 179-201. doi:10.1207/s15327841mpee1003_4

Walker, N. (2012). Throw Away The Master's Tools: Liberating Ourselves From the Pathology Paradigm. Loud Hands: autistic People,, Speaking (pp. 154-162). Washington, DC: The autistic Press.

Walmsley, J. (2004). Inclusive learning disability research: the (nondisabled) researcher's role. British Journal of Learning Disabilities, (2001), 65-71. Retrieved from http://onlinelibrary.wiley.com/doi/10.1111/j.1468-3156.2004.00281.x/full

Wehmeyer, M, Bersani, H., \& Gagne, R. (2000). Riding the Third Wave SelfDetermination and Self-Advocacy in the 21st Century. Focus on Autism and Other .... Retrieved from http://foa.sagepub.com/content/15/2/106.short 
Wehmeyer, M. L. (2005). Self-Determination and Individuals with Severe Disabilities: Reexamining Meanings and Misinterpretations. Research and Practice for Persons with Severe Disabilities, 30(3), 113-120. doi:10.2511/rpsd.30.3.113

Wehmeyer, M. L., Abery, B. H., Zhang, D., Willis, D., Hossain, W. A., Ball, A., Bacon, A., et al. (2011). Personal Self-Determination and Moderating Variables that Impact Efforts to Promote Self-Determination. Exceptionality, (October 2012), 19-30.

Wehmeyer, M. L., Shogren, K. A., Zager, D., Smith, T. E. C., \& Simpson, R. (2010). Research-Based Principles and Practices for Educating Students with Autism : SelfDetermination and Social Interactions, 45(4), 475-486.

Wehmeyer, Michael, \& Schwartz, M. (1998). The Relationship Between SelfDetermination and Quality of Life for Adults with Mental Retardation, 33(1), 3-12.

Wellman, B., \& Gulia, M. (1999). Net-Surfers Don't Ride Alone: Virtual Communities as Communities. Networks in the Global Village: Life in Contemporary Communities (pp. 331-366). Boulder, CO: Westview Press.

Whitney, C. (2006). Intersections in Identity-Identity Development among Queer Women with Disabilities. Sexuality and Disability, 24(1), 39-52. doi:10.1007/s11195-005-9002-4

Whyte, W. F. (1991). Participatory Action Research. Thousand Oaks, CA: Sage Publications, Inc.

Williams, D. (1992). Nobody Nowhere. New York, NY: Times Books.

Williams, D. (1994). Somebody Somewhere. New York, NY: Three Rivers Press.

Williams, G. C., McGregor, H. a, Sharp, D., Levesque, C., Kouides, R. W., Ryan, R. M., \& Deci, E. L. (2006). Testing a self-determination theory intervention for motivating tobacco cessation: supporting autonomy and competence in a clinical trial. Health psychology : official journal of the Division of Health Psychology, American Psychological Association, 25(1), 91-101. doi:10.1037/0278-6133.25.1.91

Williams, G., Freedman, Z. R., \& Deci, E. (1998). Supporting Autonomy to Motivate Patients with Diabetes for Glucose Control. Diabetes Care, 21(10), 1644-1652.

Wing, L. (1997). Syndromes of Autism and Atypical Development. In D. J. Cohen \& F. Volkmar (Eds.), Handbook of Autism and Pervasive Developmental Disorders (Second edi., pp. 148-172). New York: Wiley. 
Wing, L., Gould, J., \& Gillberg, C. (2011). Autism spectrum disorders in the DSM-V: better or worse than the DSM-IV? Research in Developmental Disabilities, 32, 768-773.

Wing, L., \& Potter, D. (2002). The epidemiology of autistic spectrum disorders: Is the prevalence rising? Mental Retardation and Developmental Disabilities Research Reviews, 8, 151-161.

Woodbury-Smith, M. R., Robinson, J., Wheelwright, S., \& Baron-Cohen, S. (2005). Screening adults for Asperger Syndrome using the AQ: A preliminary study of its diagnostic validity in clinical practice. Journal of Autism and Developmental Disorders, 35(3), 331-335.

Yergeau, M. (2010). Circle Wars: Reshaping the Typical Autism Essay. Disability Studies Quarterly, 30(1). Retrieved from http://dsq-sds.org/article/view/1063/1222

Zickuhr, K., \& Smith, A. (2012). Digital differences. Retrieved from http://pewlnternet.org/ /media/Files/Reports/2012/PIP_Digital_differences_0413 12.pdf 